Florida International University FIU Digital Commons

$10-29-2012$

\title{
Design Optimization of Modern Machine-drive Systems for Maximum Fault Tolerant and Optimal Operation
}

\author{
Ali Sarikhani \\ Florida International University, asari001@fiu.edu
}

DOI: $10.25148 /$ etd.FI12120407

Follow this and additional works at: https://digitalcommons.fiu.edu/etd

\section{Recommended Citation}

Sarikhani, Ali, "Design Optimization of Modern Machine-drive Systems for Maximum Fault Tolerant and Optimal Operation" (2012). FIU Electronic Theses and Dissertations. 766.

https://digitalcommons.fiu.edu/etd/766 


\title{
FLORIDA INTERNATIONAL UNIVERSITY
}

Miami, Florida

\section{DESIGN OPTIMIZATION OF MODERN MACHINE DRIVE SYSTEMS FOR MAXIMUM FAULT TOLERANT AND OPTIMAL OPERATION}

\author{
A dissertation submitted in partial fulfillment of \\ the requirement for the degree of \\ DOCTOR OF PHILOSOPHY \\ in \\ ELECTRICAL ENGINEERING \\ by
}

Ali Sarikhani 


\section{To: Dean Amir Mirmiran}

\section{College of Engineering and Computing}

This dissertation, written by Ali Sarikhani, and entitled design optimization of modern machine-drive systems for maximum fault tolerant and optimal operation, having been approved in respect to style and intellectual contents, is referred to you for judgment.

We have read this dissertation and recommend that is be approved.

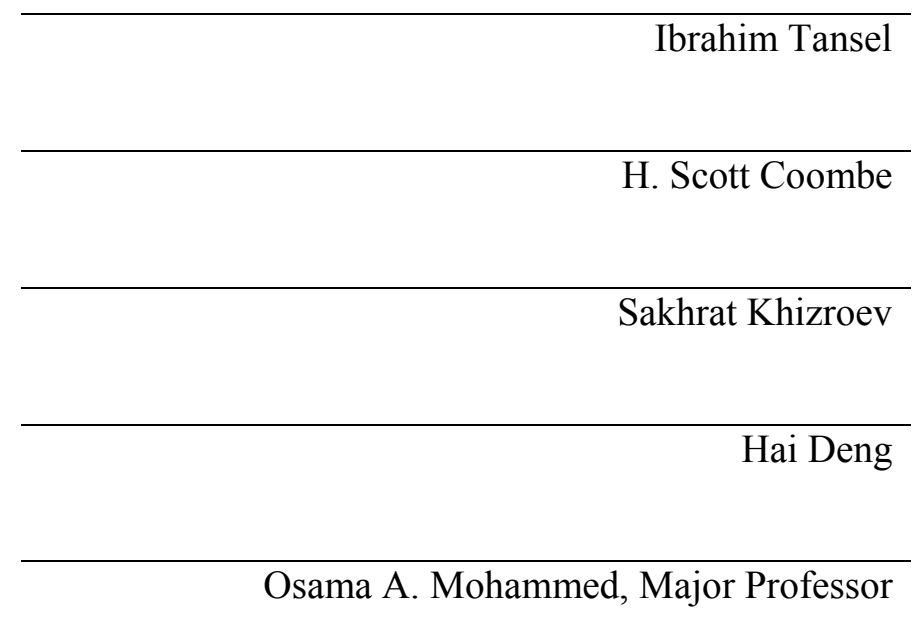

Osama A. Mohammed, Major Professor

Date of Defense: Octobor 29, 2012

The dissertation of Ali Sarikhani is approved.

Dean Amir Mirmiran

College of engineering and Computing

Dean Lakshmi N. Reddi

University Graduate School

Florida International University, 2012 
(C) Copyright 2012 by Ali Sarikhani

All rights reserved 


\section{DEDICATION}

This dissertation is dedicated to my supportive wife, Nooshin Nabizadeh, and to my always encouraging and ever faithful parents, Narges Ghasemi and Mahmoud Sarikhani. 


\section{ACKNOWLEDGMENTS}

I owe several years of my research life to my advisor Professor Osama A. Mohammed for his willingness to accept me in his laboratory and for providing me with a great research motivations, and research facilities. Hence, I wish to express in words my deepest gratitude to my adviser, Professor Osama Mohammed for his guidance, encouragement and support during my graduate study. His creative thinking, knowledge and expertise on computational electromagnetic were indeed the fuse of my propulsion motor in conducting this research.

I would like to thank my dissertation committee members Professor Sakhrat Khirzroev, Professor Ibrahim Tansel, Professor Hai deng, and Dr. H. Scott Coombe for their insightful comments and constructive suggestions in the review of my research proposal and dissertation.

I would like to acknowledge the partial research support provided from the office of Naval Research and the US department of Energy throughout my years of research in Energy Systems Laboratory. I also acknowledge the dissertation year fellowship from FIU graduate school during the last year of my studies at Florida International University.

Although this doctoral dissertation is considered to be my personal accomplishment; I would like to acknowledge my colleagues in Energy Systems Research Laboratory for their creative support, encouragement, and collaborations during my graduate studies.

I also appreciate the trust and support of Maria Benincasa, Pat Brammer, Ana Saenz, and Oscar Silveria during my studies in department of electrical and computer engineering. 


\section{ABSTRACT OF THE DISSERTATION \\ DESIGN OPTIMIZATION OF MODERN MACHINE DRIVE SYSTEMS FOR MAXIMUM FAULT TOLERANT AND OPTIMAL OPERATION \\ by}

\section{Ali Sarikhani}

Florida International University, 2012

Miami, Florida, USA

Professor Osama A. Mohammed, Major Professor

Modern electric machine drives, particularly three phase permanent magnet machine drive systems represent an indispensable part of high power density products. Such products include; hybrid electric vehicles, large propulsion systems, and automation products. Reliability and cost of these products are directly related to the reliability and cost of these systems. The compatibility of the electric machine and its drive system for optimal cost and operation has been a large challenge in industrial applications. The main objective of this dissertation is to find a design and control scheme for the best compromise between the reliability and optimality of the electric machine-drive system. The effort presented here is motivated by the need to find new techniques to connect the design and control of electric machines and drive systems.

A highly accurate and computationally efficient modeling process was developed to monitor the magnetic, thermal, and electrical aspects of the electric machine in its operational environments. The modeling process was also utilized in the design process in form finite element based optimization process. It was also used in hardware in the loop finite element based optimization process. The modeling process was later employed in the design of a very accurate and highly efficient physics-based customized observers that are required for the fault diagnosis as well the sensorless 
rotor position estimation. Two test setups with different ratings and topologies were numerically and experimentally tested to verify the effectiveness of the proposed techniques.

The modeling process was also employed in the real-time demagnetization control of the machine. Various real-time scenarios were successfully verified. It was shown that this process gives the potential to optimally redefine the assumptions in sizing the permanent magnets of the machine and DC bus voltage of the drive for the worst operating conditions.

The mathematical development and stability criteria of the physics-based modeling of the machine, design optimization, and the physics-based fault diagnosis and the physics-based sensorless technique are described in detail.

To investigate the performance of the developed design test-bed, software and hardware setups were constructed first. Several topologies of the permanent magnet machine were optimized inside the optimization test-bed. To investigate the performance of the developed sensorless control, a test-bed including a $0.25(\mathrm{~kW})$ surface mounted permanent magnet synchronous machine example was created. The verification of the proposed technique in a range from medium to very low speed, effectively show the intelligent design capability of the proposed system. Additionally, to investigate the performance of the developed fault diagnosis system, a test-bed including a $0.8(\mathrm{~kW})$ surface mounted permanent magnet synchronous machine example with trapezoidal back electromotive force was created. The results verify the use of the proposed technique under dynamic eccentricity, DC bus voltage variations, and harmonic loading condition make the system an ideal case for propulsion systems. 


\section{TABLE OF CONTENTS}

CHAPTER

PAGE

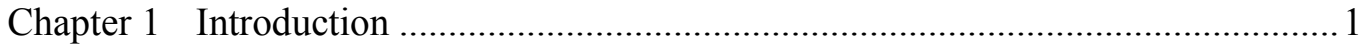

1.1 Permanent magnet synchronous machine (PMSM) ..................................... 1

1.2 Reliability and optimality in PMSM.......................................................... 2

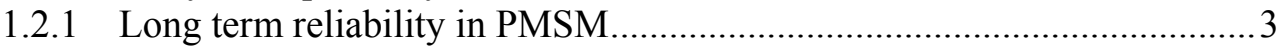

1.2.1.1 Consideration of the permanent magnet stability ............................ 3

1.2.1.2 Consideration of the design parameter in PMSM reliability .............5

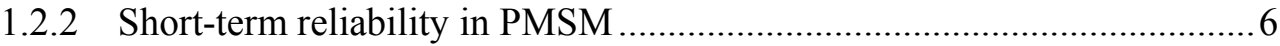

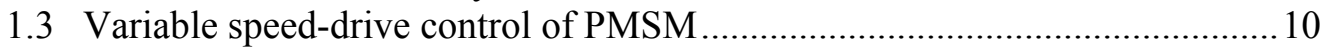

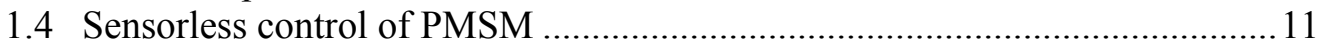

1.5 Conventional approach to the design of 3-phase PMSM-drive systems .......... 11

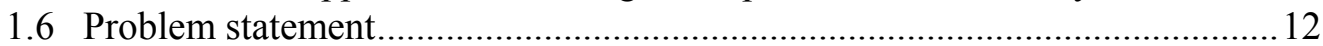

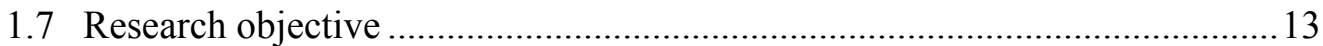

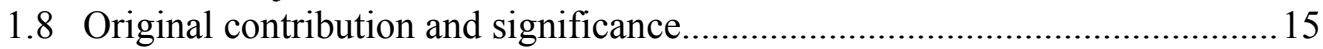

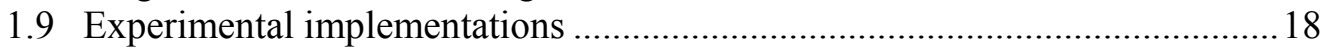

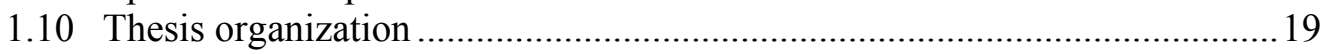

Chapter 2 Classic Design of Permanent Magnet Synchronous Machine..................2 21

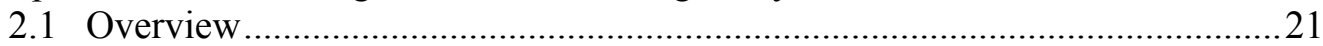

2.2 Convectional geometries in permanent magnet synchronous machine ..........223

2.3 Classic design procedure of permanent magnet synchronous machine...........26

2.3.1 Permanent magnet circuit model........................................................26

2.3.2 Magnetic circuit of the PMSM with small air gap ..............................22

2.3.3 Effect of temperature on the magnet remnance flux density ....................32

2.4 Winding arrangement for the radial flux PMSM ............................................. 33

2.4.1 Valid number of slots for specific number of magnetic poles for double

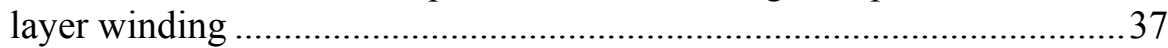

2.4.2 Valid coil span concerning number of magnetic poles .......................... 38

2.4.3 Number of coils per phase for double layer windings ............................39

2.4.4 Consideration in the design of non-overlapping windings ......................39

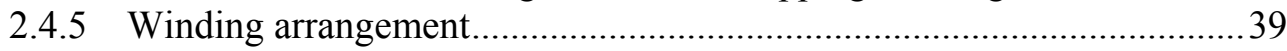

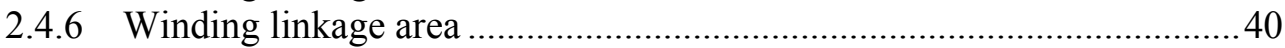

2.4.7 Other consideration for selection of the proper winding arrangement ....45

2.4.7.1 Effect of the number of the poles and slots on radial force and

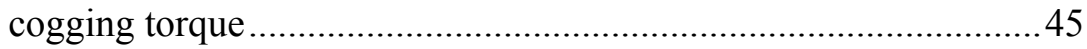

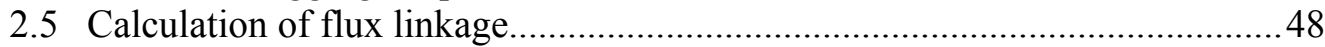

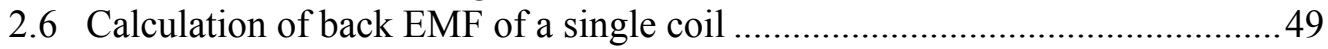

2.7 Calculation of power and torque for single coil............................................50

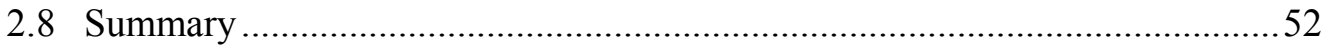

Chapter 3 Modeling of Permanent Magnet Synchronous Machine (PMSM) ...........53

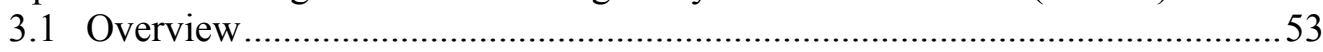

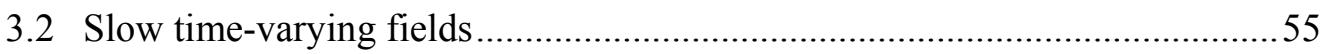

3.3 Magneto dynamic fields (low frequency quasi-stationary fields)...................55

3.3.1 Calculation of stored magnetic energy ..............................................58 


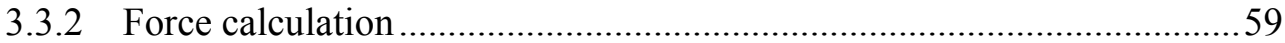

3.3.2.1 Lorentz method for calculation of force ...................................59

3.3.2.2 Maxwell stress for calculation of the force ....................................6 60

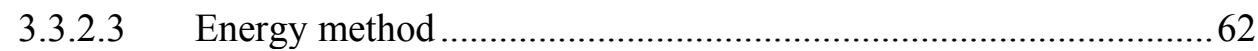

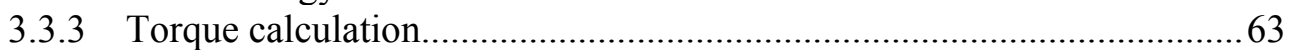

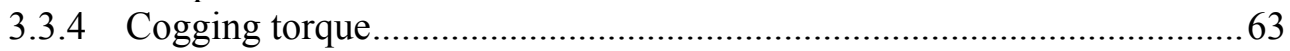

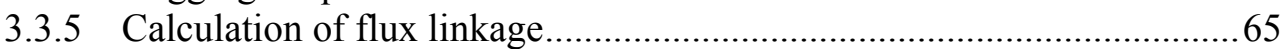

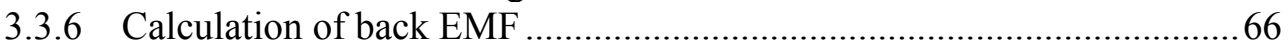

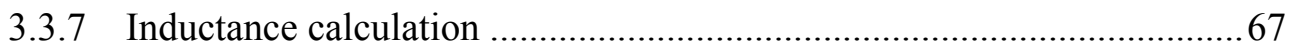

3.3.7.1 Flux linkage method for inductance calculation............................68

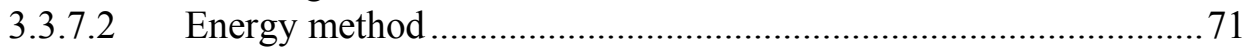

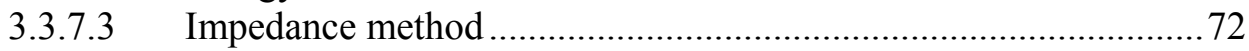

3.3.7.4 Open-circuited inductance calculation method.............................73

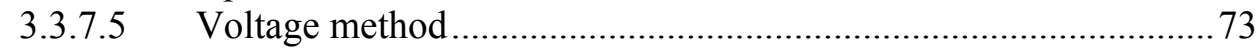

3.3.8 Coupling of slow varying field and circuit equations ........................... 74

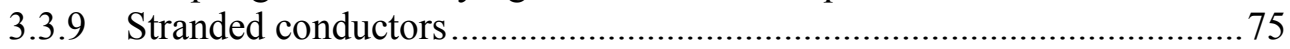

3.3.10 Boundary conditions ...................................................................... 75

3.3.11 Finite element solution for slow time-varying fields ........................... 76

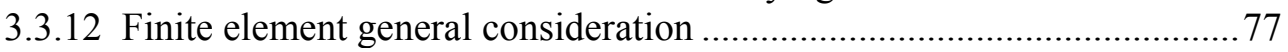

3.3.13 General consideration in using Lorenz force method .............................77

3.3.14 General consideration in using Maxwell stress tensor method................77

3.3.15 General consideration in using energy method In force calculation........ 78

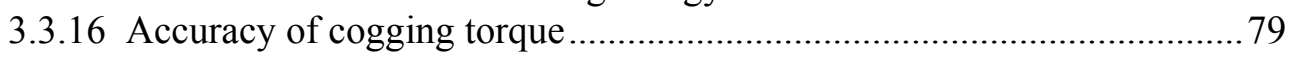

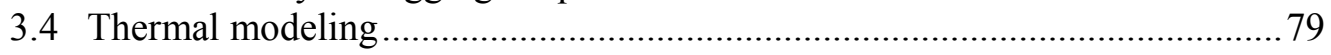

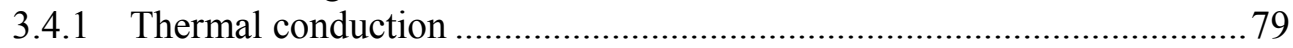

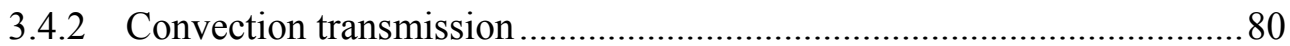

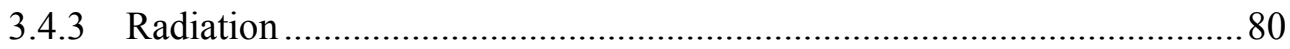

3.4.4 Analytical calculation of thermal PDE equation.................................. 81

3.4.5 Simplification conductive thermal resistance ......................................... 87

3.4.6 Complete thermal model of a PMSM ................................................ 88

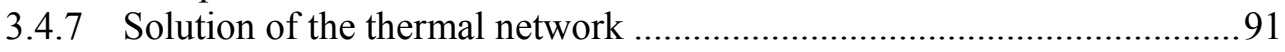

3.5 Modeling of demagnetization of magnets.................................................... 92

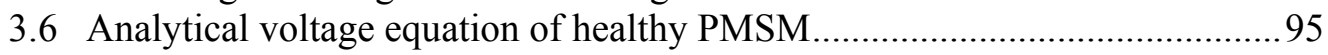

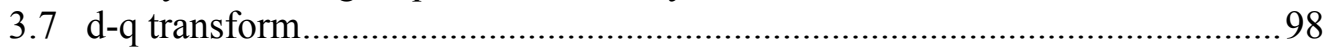

3.7.1 Transformation between reference frames......................................... 100

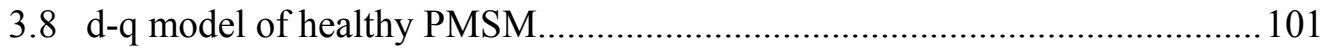

3.9 Healthy phase variable motor model in a-b-c frame of reference .................. 103

3.10 Phase variable model based line-line voltage modeling for healthy PMSM without access to neutral point ................................................................. 106

3.11 Phase variable model based line-line voltage modeling for Faulty PMSM without access to neutral point ................................................................ 107

3.12 Phase variable model based line-line voltage modeling for faulty PMSM with access to neutral point ............................................................................... 111

3.13 Thermo-electric modeling of PMSM taking into accounts the demagnetization issue .................................................................. 112

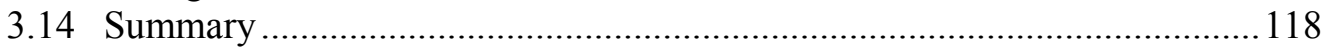


Chapter 4 Control of Permanent Magnet Synchronous Machines .......................... 119

4.1 Introduction to the PMSM-drive system................................................... 119

4.2 Voltage source inverter (VSI) drive for control of PMSM ............................ 120

4.3 Current-source inverter (CSI) drive for control of PMSM ........................... 121

4.4 Conventional CSI-based-VSI drive systems.............................................. 122

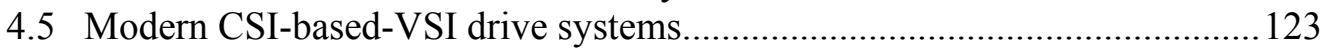

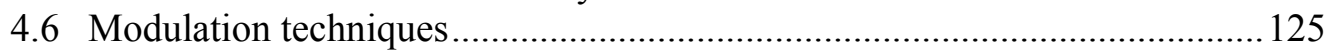

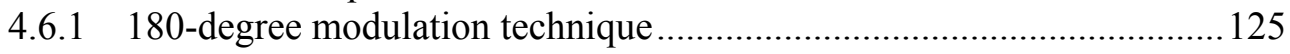

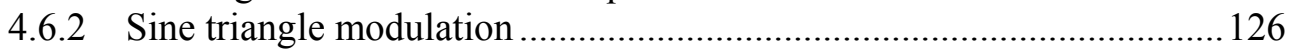

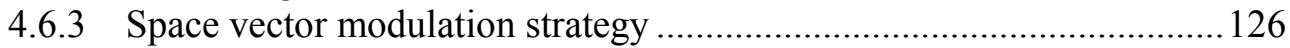

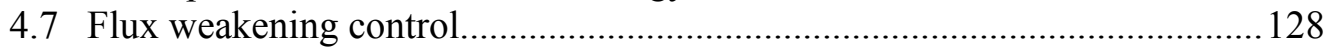

4.8 Current control in CSI-based VSI.......................................................... 130

4.9 Demagnetization control for reliable flux weakening control in PM

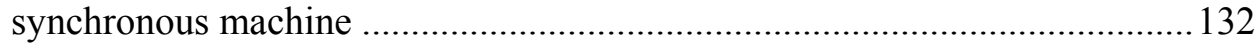

4.9.1 Design of current limiter for reliable flux weakening control ............... 134

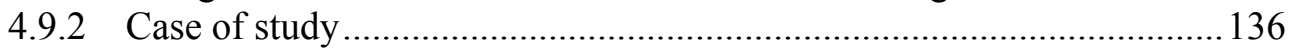

4.9.2.1 Model of the magnet ............................................................. 137

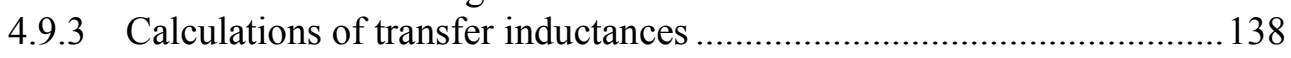

4.9.4 Calculation of operational PM temperature .......................................... 140

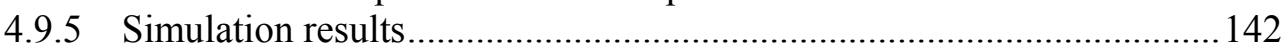

4.9.6 Demagnetization assessment in different PMSM designs ................... 150

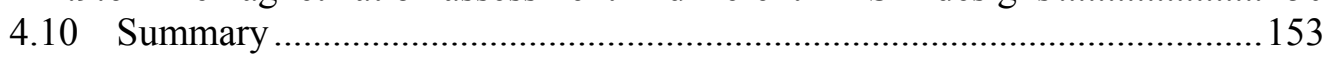

Chapter 5 Sensorless Control of PM Synchronous Machines by Physics-based EMF

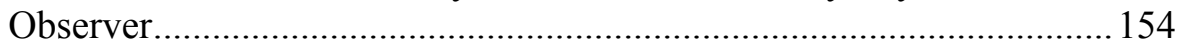

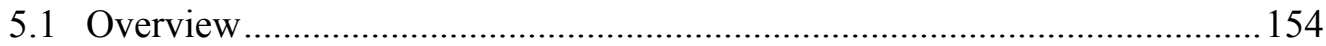

5.2 Sensorless rotor position estimation and control in PMSM.......................... 154

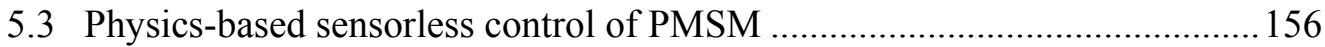

5.2.1 Adaptive angle compensator .......................................................... 160

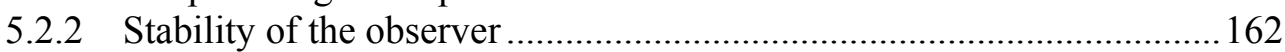

5.2.3 Calculation of the parameters for physics-based observer .................. 163

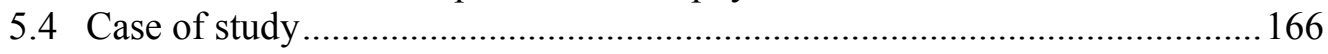

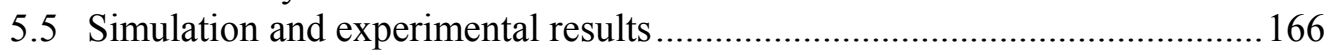

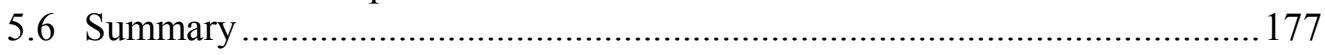

Chapter 6 Inter-turn Fault Detection in PM Synchronous Machines by Physics-based

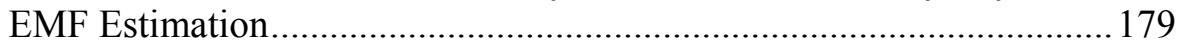

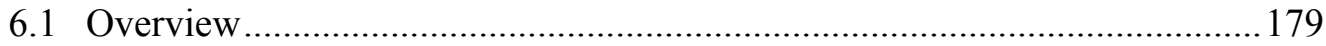

6.2 Inter-turn fault diagnosis in PMSM ....................................................... 179

6.3 Inter-turn fault modeling for 3-phase PMSM without access to neutral point

6.4 Design of EMF estimation system for inter-turn fault detection .................. 186

6.4.1 Stability criteria of the estimator...................................................... 188

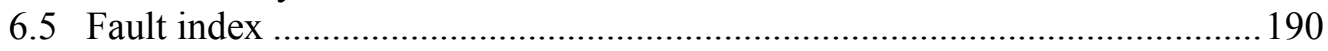

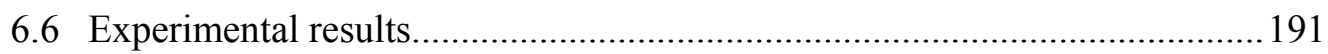

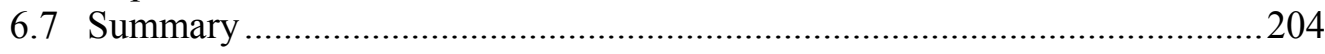


Chapter 7 Optimization of Machine Drive Systems ............................................206

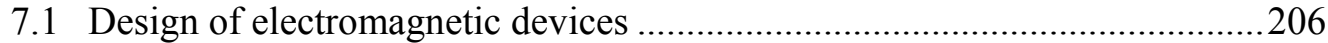

7.2 Optimization based evolutionary algorithms ...............................................208

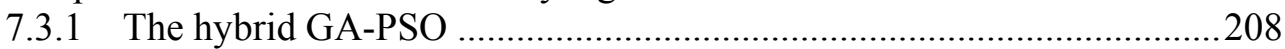

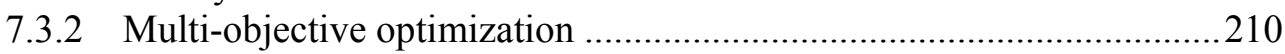

7.3.3 The discrete search space ...............................................................2211

7.3 Design improvement of PMSM-drive system using physics-based motor model

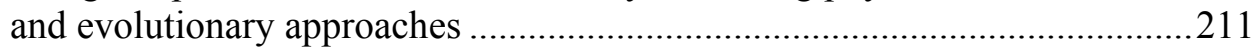

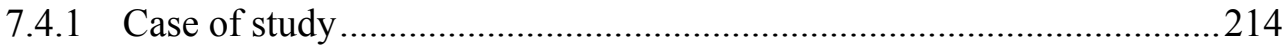

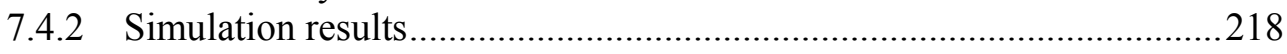

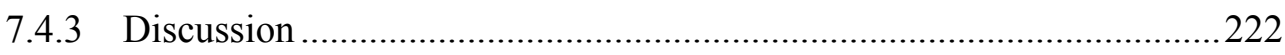

7.4 Hardware in the loop based finite element design optimization process for the computational prototyping of electric motor-drives ...................................225

7.5.1 The hardware in loop compatible physics-based modeling of the PM

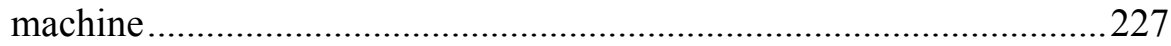

7.5.2 HIL-FE based optimization Process ...................................................228

7.5.2.1 Soft multi-objective optimization ..........................................2228

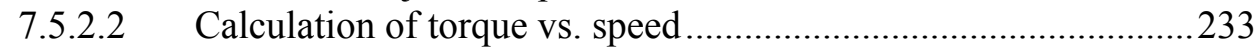

7.5.2.3 Setting of the current adjustable load (CAL) .............................233

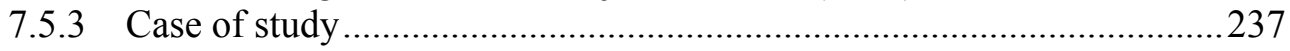

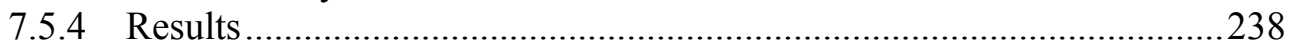

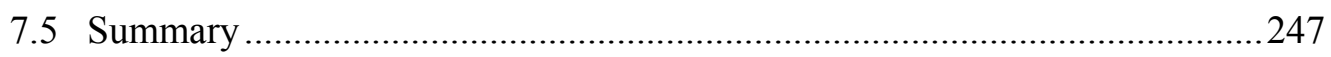

Chapter 8 Discussion of Results, Conclusions and Future Work .........................249

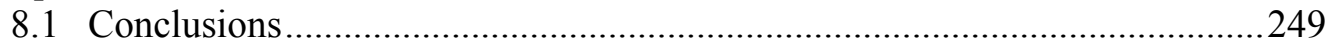

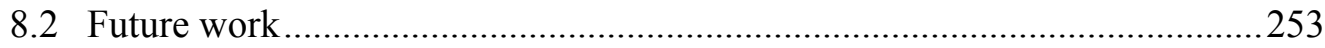

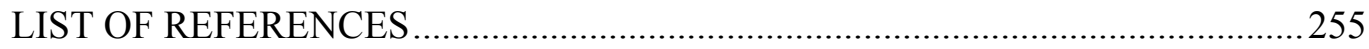

VITA

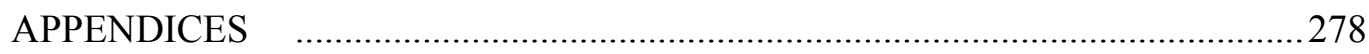




\section{LIST OF TABLES}

TABLE

Table 4.1 Winding arrangement of the studied machine 137

Table 7.1 Boundaries of optimization input variables 216

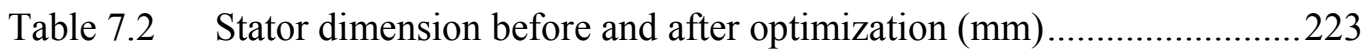

Table 7.3 Stator dimension before and after optimization $(\mathrm{mm}) \ldots \ldots \ldots \ldots \ldots \ldots \ldots \ldots . . .223$

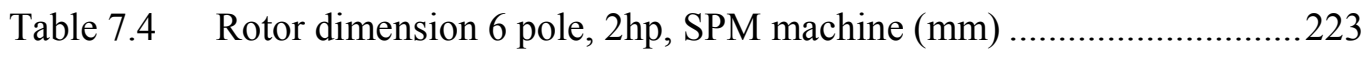

Table 7.5 Volumes and mass before and after optimization...............................224

Table 7.6 Machine performance before and after optimization...........................224

Table 7.7 current adjustable load parameters ...................................................2. 236

Table 7.8 Boundaries and constraints of optimization's input variables.............243

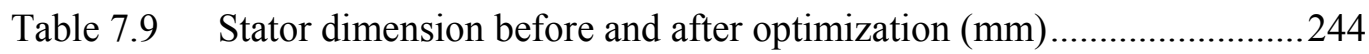

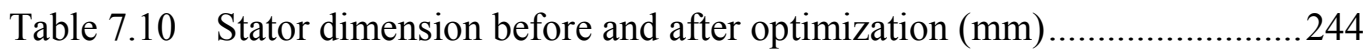

Table 7.11 Rotor dimension 12 slot/10 poles, SPM machine $(\mathrm{mm})$......................244

Table 7.12 Volumes and mass before and after optimization..............................244

Table 7.13 Average performance before and after optimization ..........................244

Table 7.14 Comparison of THD and RMS of phase current for different levels of torque and speed ......................................................................... 245 


\section{LIST OF FIGURES}

FIGURE

PAGE

Figure 2.1 Different magnet arrangement in axial flux permanent magnet machine...

Figure 2.2 Intersection of the conventional geometry for PMSM .........................25

Figure 2.3 Slot opening changes in the $5^{\text {th }}$ test set ...........................................25

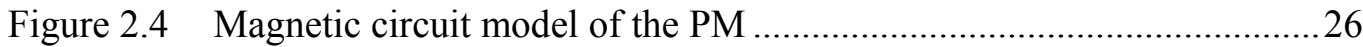

Figure 2.5 Simple geometry and flux path of the surface mount PMSM ..............27

Figure 2.6 Reluctance network of the flux path shown in Figure 2.5 .....................28

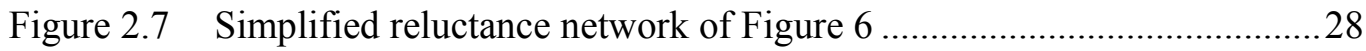

Figure 2.8 Visual illustration of the air gap flux density with respect to the magnets.

Figure 2.9 The possible demagnetization profiles.................................................. 31

Figure 2.10 The effect of the temperature on the magnets......................................33

Figure 2.11 The overlapping distributed (a), overlapping concentrated (b), nonoverlapping concentrated double layer (c), non-overlapping concentrated

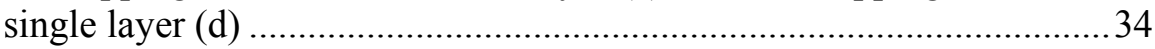

Figure 2.12 Winding linkage area of a coil for the concentrated winding with different slot pitch ............................................................................ 42

Figure 2.13 Linkage area Non-overlapping winding concentrated and distributed winding with different coil sections.

Figure 2.14 From magnet structure to back EMF creation in concentrated windings ...

Figure 3.1 T-equivalent thermal network for trapezoidal geometrical element.......8 84

Figure 3.2 Trapezoidal geometrical element of a PMSM .................................... 85

Figure 3.3 T-equivalent thermal network for cylindrical geometrical element .......86

Figure 3.4 Cylindrical geometrical element of a PMSM ..................................... 87

Figure 3.5 Simplified thermal element for the cylindrical geometrical element......88 
Figure 3.6 Healthy Transient thermal model of the machine .92

Figure 3.7 Modified transient thermal model of the windings for ISCF studies...... 92

Figure 3.8 Reversible demagnetization process of a typical sintered magnet on a $\mathrm{Nd}-\mathrm{Fe}-\mathrm{B}$ base at two temperatures (T1>T0) (a), irreversible demagnetization due to direct reverse field (b).....................................95

Figure 3.9 Typical demagnetization surface for sintered magnet on a Nd-Fe-B base, $\mathrm{B}(\mathrm{H})$ at different temperatures 95

Figure 3.10 Schematic view of a 2-pole, PMSM ......................................................96

Figure 3.11 3-phase balance winding of a PMSM …................................................96

Figure 3.12 Phasor transformation of 3-phase balance a-b-c frame to d-q frame of reference 100

Figure 3.13 Transformation between two dq0 $\mathrm{s}^{\mathrm{x}}$ and $\mathrm{dq} 0 \mathrm{~s}^{\mathrm{y}}$ frame of reference ....... 101

Figure 3.14 Inter-turn for conventional PMSM drives supplied by a 3-phase inverter . 111

Figure 3.15 Inter-turn fault model for conventional fault tolerant machines supplied by a three single H-bridge inverters ............................................... 112

Figure 3.16 Thermo-electric modeling of PMSM taking into accounts the

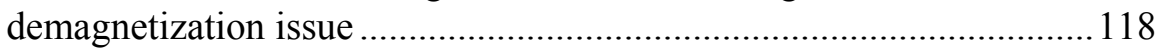

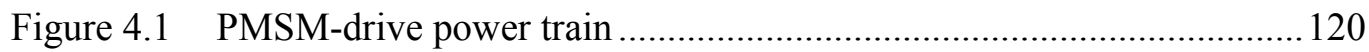

Figure 4.2 Voltage source inverter (VSI) drive for control of PMSM ................... 121

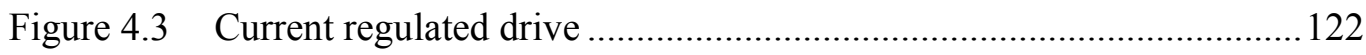

Figure 4.4 Current regulator based voltage source inverter ................................. 123

Figure 4.5 Detailed PMSM-VSI drive with current control loop with additional control and diagnosis systems .......................................................... 124

Figure 4.6 Demagnetization control in PMSM based current regulated drive....... 135

Figure 4.7 Schematic diagram of current limiter ............................................. 136

Figure 4.8 Rotor geometry (a) dimension (b) of V-type PMSM, artificial coil C1$\mathrm{C} 1$ ' is located on flux barrier areas of M2

Figure 4.9 Typical demagnetization surface for sintered magnet on a Nd-Fe-B base, $\mathrm{B}(\mathrm{H})$ at different temperatures 138 
Figure 4.10 The closed form 3-D shape of the Figure 4.9

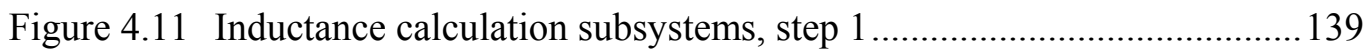

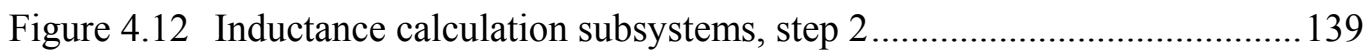

Figure 4.13 Geometry and dimension of the studied machine in $(\mathrm{mm}) \ldots \ldots \ldots \ldots \ldots \ldots . . . . .141$

Figure 4.14 Transient thermal model of the stator ............................................... 141

Figure 4.15 Transient thermal model of the rotor for the V-type PMSM ................ 141

Figure 4.16 Relation between remnant flux density of the studied PM and temperature at $T_{0}=0^{\circ}(C), \mathrm{H}=0(\mathrm{~A} / \mathrm{m})(\mathrm{a})$, remnant flux density of magnet versus temperature at knee point (b) Flux density function on the face of magnet $M_{2}$ as a function of $i_{d}(\mathrm{c})$, Minimum flux density function on the face of $M_{2}$ as a function of $i_{d}(\mathrm{~d})$..... 143

Figure 4.17 $E_{a b c}$ (a), $\lambda_{\text {Rabc }}(\mathrm{b}), T_{c o g}(\mathrm{c})$, and $B_{m r}(\mathrm{~d})$, calculated from a non-linear transient FE analysis with motion phase " $\mathrm{b}$ " 143

Figure 4.18 Mutual inductance between armature phase "a" and the magnet artificial coil $\mathrm{C}-\mathrm{C}^{\prime}$ on $\mathrm{M}_{2}$ at different current status. 144

Figure 4.19 Self-inductance of armature phase "a" at different current status ........ 144

Figure 4.20 Mutual inductance between armature phase " $a$ " and the armature....... 144

Figure 4.21 Scenario1: Irreversible and progressive demagnetization, the current

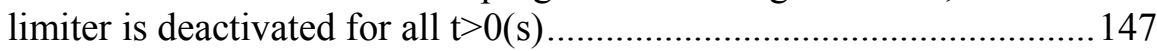

Figure 4.22 Scenario2: short-term Irreversible demagnetization, the current limiter is deactivated for all $\mathrm{t}>0(\mathrm{~s})$

Figure 4.23 Scenario3: short-term Irreversible demagnetization, the current limiter is activated for all $\mathrm{t}>0(\mathrm{~s})$ 148

Figure 4.24 Absolute flux density on the magnets for the V-type PMSM for $\left(i_{d}^{*}, i_{q}^{*}\right)=$ $(-25,0)$ and $T_{\text {magnet }}=20^{\circ}(C)$, and for $\left(i_{d}^{*}, i_{q}^{*}\right)=(0,0)$ and $T_{\text {magnet }}=20^{\circ}(C)$ 149

Figure 4.25 magnetic flux picture (b) for the V-type PMSM for $\left(i_{d}^{*}, i_{q}^{*}\right)=(-25,0)$ and $T_{\text {magnet }}=20^{\circ}(C)$. 149

Figure 4.26 Rotor geometry of double PMSM, artificial coil $\mathrm{C} 1-\mathrm{C} 1$ ' to $\mathrm{C} 4-\mathrm{C} 4$ ' is 
located on flux barrier areas of $\mathrm{M}_{1}$ to $\mathrm{M}_{4}(\mathrm{~b})$, equivalent geometry of double PMSM (b1) 150

Figure 4.27 Rotor geometry of flat PMSM, artificial coil C1-C1' is located on flux barrier areas of $\mathrm{M}_{1}(\mathrm{c})$ : equivalent geometry of flat PMSM (c1) ..........150

Figure 4.28 The dimension of the U-type and the flat PMSM.............................. 151

Figure 4.29 Irreversible and not progressive demagnetization .............................. 151

Figure 4.30 Irreversible and not progressive demagnetization ............................... 152

Figure 4.31 Magnetic flux picture for the three PMSM in (a), (b), and (c), and absolute flux density on the magnets in (a1), (b1), (c1). All for the $\left(i_{d}^{*}\right.$,

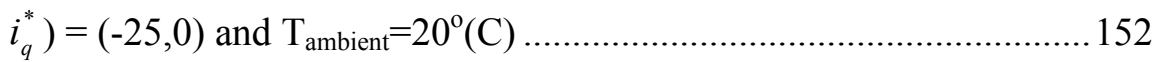

Figure 5.1 Hysteresis EMF estimator subsystem ............................................... 164

Figure 5.2 Adaptive angle compensator subsystem .......................................... 164

Figure 5.3 Combination of Physics-based PM circuit model, adaptive angle compensator, and hysteresis estimator subsystems ............................. 164

Figure 5.4 Geometry $(\mathrm{Cm})$ and winding arrangement of the studied machine...... 167

Figure 5.5 Sensorless speed control in Simulink domain ..................................... 168

Figure 5.6 Calculated self-inductance at five saturation level ............................. 168

Figure 5.7 Calculated mutual inductance at five saturation level ........................ 168

Figure 5.8 Flux linkage and cogging torque of the studied machine .................... 169

Figure 5.9 Estimated versus measured speed changes due to Step change in reference speed for 20\% nominal load (simulation) ............................ 169

Figure 5.10 Estimated versus measured speed changes due to Step change in reference speed for 20\% nominal load (experimental) ........................ 169

Figure 5.11 Rotor position changes due to Step change in reference speed for $20 \%$ nominal load (Simulation) ............................................................... 170

Figure 5.12 Rotor position changes due to Step change in reference speed at $20 \%$ nominal load (Experimental) ....................................................... 170

Figure 5.13 Phase current changes due to Step change in reference speed under $20 \%$ nominal load (Experimental)

Figure 5.14 EMF changes due to Step change in reference speed under 20\% nominal 
load (Experimental)

Figure 5.15 Operational inductance due to Step change in reference speed under 20\% nominal load (Experimental)

Figure 5.16 Presented error due to Step change in reference speed under $20 \%$ nominal load (Experimental)

Figure 5.17 Rotor position for reference speed of $0.5(\mathrm{rad} / \mathrm{s})$ under nominal load (Experimental) 173

Figure 5.18 Estimated EMF for reference speed of $0.5(\mathrm{rad} / \mathrm{s})$ under nominal load (Experimental) 173

Figure 5.19 Presented error $(\%)$ for the reference speed of $0.5(\mathrm{rad} / \mathrm{s})$ under nominal load (Experimental) 174

Figure 5.20 Rotor position changes due to Step change in load from $20 \%$ to $100 \%$ of nominal load at $0.2(\mathrm{~s})$ for reference speed of $20(\mathrm{rad} / \mathrm{s})$ (Experimental)...

Figure 5.21 Operational inductance profile for Step change in load from $20 \%$ to $100 \%$ of nominal load at 0.2 (s) for reference speed of $20(\mathrm{rad} / \mathrm{s})$ (Experimental) 174

Figure 5.22 Current changes due to Step change in load from $20 \%$ to $100 \%$ of nominal load at $0.2(\mathrm{~s})$ for reference speed of $20(\mathrm{rad} / \mathrm{s})$ (Experimental)...

Figure 5.23 Operation of the sensorless operation under $20 \%$ nominal load under sudden voltage interruption to zero at $0.2<\mathrm{t}<0.21$ for speed of $20(\mathrm{rad} / \mathrm{s})$

Figure 5.24 Initial transient during rotor position estimation 176

Figure 5.25 Rotor position for very slow reference speed reversal from 3 to $-3(\mathrm{rad} / \mathrm{s})$ under nominal load (Experimental) 176

Figure 5.26 presented error due to very slow reference speed reversal from 3 to -3 $(\mathrm{rad} / \mathrm{s})$ under nominal load (Experimental).

Figure 5.27 Rotor position for fast reference speed reversal from -3 to $3(\mathrm{rad} / \mathrm{s})$ under nominal load (Experimental) 177

Figure 5.28 Presented error due to fast reference speed reversal from -3 to $3(\mathrm{rad} / \mathrm{s})$ under nominal load (Experimental)

Figure 6.1 Schematic diagram of equation (6-5).............................................. 188

Figure 6.2 Schematic diagram of the whole EMF-based diagnosis system 188 
Figure 6.3 Step response of Pade approximation of time delay $\left(e^{-T_{d} \cdot s}\right)$.........

Figure 6.4 The geometry of the machine in (cm) and the winding arrangement... 193

Figure 6.5 flux linkage(a), Cogging Torque (b), $\mathrm{L}_{\mathrm{aa}}$ at different saturation levels(c), and $\mathrm{L}_{\mathrm{ab}}$ at different saturation levels $(\mathrm{d})$

Figure 6.6 Reference versus estimated EMF in faulty phase with different fault intensity, NF (a), LF (b), MF (c), HF (d), all for speed of $0.75 \mathrm{rad} / \mathrm{s} \ldots 198$

Figure 6.7 Reference versus estimated EMF in faulty phase with different fault intensity, NF (a), LF (b), MF (c), HF (d) all for speed of $2 \mathrm{rad} / \mathrm{s} \ldots \ldots . . .198$

Figure 6.8 Reference versus estimated EMF in faulty phase with different fault intensity, NF (a), LF (b), MF (c), HF (d) all for speed of $4 \mathrm{rad} / \mathrm{s}$......... 199

Figure 6.9 Reference versus estimated EMF in faulty phase with different fault intensity, H (a), LF (b), MF (c), HF (d) all for speed of $8 \mathrm{rad} / \mathrm{s}$

Figure 6.10 Reference versus estimated EMF with MF fault intensity at phase "b" for the speed of $5 \mathrm{rad} / \mathrm{s}$ 200

Figure 6.11 Comparison of estimated versus reference EMF for a harmonic load 200

Figure 6.12 Reference versus estimated EMF for a transient HF intensity 200

Figure 6.13 Transient fault index for two successive LF situations at 2(s) and 6(s) for case 1 and 2 .both for $25 \%$ of nominal torque 201

Figure 6.14 Voltage terminal and phase current for LF intensity condition at two different speed $0.75(\mathrm{rad} / \mathrm{s})(\mathrm{a}, \mathrm{b})$, and $8(\mathrm{rad} / \mathrm{s})(\mathrm{c}, \mathrm{d})$ 201

Figure 6.15 Reference versus estimated EMF during fast and slow speed variations, (a) and (b) experimental results, (c) and (d) simulation results 202

Figure 6.16 Reference versus estimated EMF in faulty phase with different fault intensity, H (a), LF (b), MF (c), HF (d) all for speed of $8 \mathrm{rad} / \mathrm{s}$ 203

Figure 6.17 Estimated electrical resistance as a function of speed and fault intensity for no load (circles) and 25\% of nominal load (stars) conditions.........203

Figure 6.18 Fault index as a function of speed and fault intensity all for $25 \%$ of nominal torque

Figure 7.1 Computational design prototyping of electromagnetic devices[194] ...208

Figure 7.2 Schematic view of the multi-objective field-performance optimization .... 
Figure 7.3 Back EMF of the phase A before and after optimization .....................220

Figure 7.4 The cogging torque in initial and optimized design...........................220

Figure 7.5 Self and mutual inductances before and after optimization.................220

Figure 7.6 Flux linkage of the phase A before and after optimization...................221

Figure 7.7 Total torque before and after optimization ......................................221

Figure 7.8 Speed before and after optimization ..............................................221

Figure 7.9 The current-back EMF waveforms before (a) and after optimization (b) ..

Figure 7.10 The current-back EMF waveforms at the vicinity of the peak of the back EMF before (a) and after optimization (b) ......................................224

Figure 7.11 The Current waveforms before (a) and after optimization (b).............225

Figure 7.12 Online HIL finite element based design optimization process .............239

Figure 7.13 Back EMF of the phase A before and after optimization at speed of 1 $(\mathrm{rad} / \mathrm{s})$

Figure 7.14 The cogging torque in initial and optimized design...........................241

Figure 7.15 Self inductances before and after optimization.................................241

Figure 7.16 Mutual inductances before and after optimization.............................242

Figure 7.17 Flux linkage of the phase "a" before and after optimization ................242

Figure 7.18 Dynamic torque measured for $0.5(\mathrm{~N}-\mathrm{m})$ and $150(\mathrm{Rad} / \mathrm{s}) \ldots \ldots \ldots \ldots \ldots \ldots . .242$

Figure 7.19 Speed dynamic measured for $0.5(\mathrm{~N}-\mathrm{m})$ and $150(\mathrm{Rad} / \mathrm{s})$..................243

Figure 7.20 Visual comparison of the reference and current adjustable load current for optimized (b) machine at $0.5(\mathrm{~N}-\mathrm{m})$ and $300(\mathrm{rad} / \mathrm{s}) \ldots \ldots \ldots \ldots \ldots \ldots \ldots . . . . . . . . . .243$

Figure 7.21 Torque Vs. speed profile, initial design (star), optimal design (square).....

Figure 7.22 The normalized current and back EMF, initial (a) and optimized (b) ..246

Figure 7.23 3-D view of the initial (a) and the optimized machine(b)...................246

Figure 7.24 Field picture and absolute flux density of the initial and the optimized 
Figure 7.25 Field picture and absolute flux density of the initial and the optimized machine in the inter-pole angle areas..............................................247 


\section{LIST OF ACRONYMS}

3-D FE

2-D FE

EMF

GCD

PMSM

SMPMSM

VSI

CSI

PWM

SVPWM

GA

PSO

AI

ISCF

NF

LF

MF

HF
Three dimentaional finite element

Two dimentional finite element

Back electromotive force

Greatest common divider

Permanent magnet synchronous machine

Surface mount permanent magnet synchronous machine

Voltage regulated (voltage source) inverter Current regulated (current source) inverter

Pulse width modulation

Space vector pulse width modulation

Genetic algorithm

Particle swarm Optimization

Artificial intelligence

Inter-turn short circuit fault

No fault

Low fault

Medium fault

High fault 


\section{Chapter 1 Introduction}

\subsection{Permanent magnet synchronous machine (PMSM)}

In the recent decades, the development of the vector control of PMSM for wide speed range in addition to the continuous cost reduction of magnetic materials with high energy density has made the AC drives based on PMSM more attractive and competitive. In addition to these the fast dynamic response, high power density and wide operating speed range has opened up new possibilities for large-scale application of PMSM [1-3].

In general, PM synchronous machines can have either sinusoidal back electromotive force (EMF) or non-sinusoidal EMF (normally trapezoidal). The EMF waveform is related to the shape and the location of the magnetic poles, the magnetization direction of the magnet (parallel, sinusoidal, radial, radial sine), the number of slot/poles and the winding arrangement. The PMSM with approximately sinusoidal back electromotive force (EMF) can be categorized either as interior permanent magnet machines (IPM) or as the surface-mounted permanent magnet machines (SPM) [4]. In IPM machines, magnets are buried inside the rotor. Consequently, in most of the IPM topologies the magnetic reluctance of the direct and quadrature magnet fluxes are not equal. This effect is called magnetic saliency, although the IPM rotor face is physically smooth. The magnetic saliency is the source of reluctance torque. This makes the IPM machines superior to the SPM machines in many applications. In SPM machines the magnets are located on the face of the rotor. Because the permeability of the magnets is close to one, the SPM machine can be considered to have a large and uniform air gap. This property makes the magnetic saliency almost negligible. As a result, the only source of the torque in SPM machine 
is magnetic torque[5]. It is created from the interaction of the magnet rotor flux and the armature flux which are perpendicular to each other. Mechanically, the IPM has more robust and solid structure than SPM since the magnets are physically buried inside the rotor iron. In addition, due to their saliency, the IPM motors are more suitable for the applications that require constant power at higher speeds than nominal speed and require flux weakening control in sensitive critical applications $[3,6]$.

\subsection{Reliability and optimality in PMSM}

A continuous growth in the usage of PMSM drives will surely be witnessed in the near future. Despite of all the positive advantages that a PMSM offers, the high power density of permanent magnet compared to the armature winding power density beside the demagnetization of permanent magnet due to reverse field and the overtemperature have made the reliability assessment, remedial action areas of the necessary issues to prevent machine collapse during internal fault or unexpected overloading conditions[7]. The demagnetization of the magnets and the progressive interturn fault are of the main electrical treats that need careful consideration during the design stage and the real time operation of the machine. The design stage takes responsibility for the long term reliability of PMSM through reliability assessment policies defined in terms of long term demagnetization, maximum flux weakening capability, thermal constraints[8]. The real-time fault diagnosis, prognostics systems and the real-time remedial strategies take responsibility for the short term reliability of PMSM-drive system. The long term reliability ensures the users that the machine will handle a worse condition for a certain periods of time. The short-term reliability tries

to apply the fault detection and remedial action in the defined time span. The interaction of short term and long term reliability is obvious. Whenever the attention to the long term reliability is increased, the short-term reliability needs less attention 
and vice-versa. The higher attention to the long term reliability increase the size/ (KW) will result in higher final cost (\$) of the PMSM. Consequently, it is seen that there is an interesting challenges between the optimality and the reliability of the PMSM-drive through the short-time reliability assessments[3], [9-12].

\subsubsection{Long term reliability in PMSM}

The long term reliability policies is defined as all the design policies that lead to more stable electrical machine against mechanical, electrical and thermal stress[1315]. The consideration of the permanent magnet stability either through the conservative choice of the permanent magnet material or through the shaping and sizing of the permanent magnet used in the PMSM are of the important issues in long term demagnetization assessment[16]. In addition, the thermal analysis, the saliency ratio, assigning the proper ratio between power density of the PM and the power density of the windings are of the most important issues that reflect the designer thought into the long term reliability of the PMSM [17].

\subsubsection{Consideration of the permanent magnet stability}

The permanent magnet stability is the capability of a permanent magnet to maintain an external magnetic field resulted from small magnetic domains locked in position by crystal anisotropy within the magnet material. The Factors that affects the magnet stability include temperature, time, adverse fields, reluctance changes, radiation, shock, stress, and vibration[9], [18-21].

The effect of time on demagnetization processis reviewd in [22]. In general, when the operating condition of PM machine is normal, the influence of time on modern permanent magnet materials is negligible. Life assessment investigations have shown that permanent magnets will see changes immediately after magnetization. Long-term time versus flux investigations have also shown that a newly magnetized magnet will 
lose a minor percentage of its flux density as a function of age. However, the life of a permanent magnet depends on many factors. Physically occurring forces tends to knock the little domains out of their normal alignment. But this is usually a very slow process. Temperature is a main factor in this process. The higher the temperature, the faster this process will happen. Heating up the magnet higher than the Curie point will immediately randomize the domains. A sharp blow can also knock domains out of their normal alignments, as can other nearby magnetic or electrical fields. Radiation can also knock domains out of their normal alignment.

The effect of reluctance change on demagnetization process is reviewd in[1]. It is recognized that, any permeance changes such as changes in air gap dimensions throughout operation can diverge the magnets from their normal condition, such as falling the magnet's operating point to below the knee of the curve, and causing partial and/or irreversible losses. This behavior of magnets is known as reluctance change. In this work this effect is not considered in the modeling, however for a comprehensive study in the fault tolerance researches this effect should be added to the physics-based model.

The thermal effect on demagnetization process is reviewd in [17]. To some extent, demagnetizing a magnet by exposure to elevated temperatures in a controlled manner stabilizes the magnet with respect to temperature. The slight reduction in flux density also improves the magnets stability because domains with low commitment to orientation are the first to lose their orientation. On the other hand, Thermal shock when permanent magnets are exposed to high temperature gradients can lead to internal fractures within the PM material and should be prevented. In general, for life assessment studies the effect of temperature on magnets can be expressed in three 
category, Reversible losses, Irreversible but recoverable losses, and Irreversible and unrecoverable losses.

The effect of reverse field on demagnetization process is reviewd in in [22]. The external reverse magnetic field in repulsion mode lead to the permanent magnet demagnetization if the operating point of the magnet drops below the knee point

\subsubsection{Consideration of the design parameter in PMSM reliability}

Ratio between the power density of the PM and the power density of the windings is an important factor in reliability of a PMSM. The lower ratio of power density of the PM to the power density of the winding can lead to the higher flux weakening capability. The higher flux weakening capability, the more effective the fault remedial action will be, and the higher demagnetization risk will be [1].

The thermal issues [23] are the main reason for electrical insulation deterioration, electrical resistance changes, and magnet demagnetization. The current density of the windings and the power density of the armature windings are the most important factors that reflect the thermal aspect of the machine to the machine performance.

The saliency ratio[24] is defined as the ratio of the quadrature to the direct inductance path. For SPM machines it is very close to one and for the IPM machines it is normally lower than one. It can also be higher than one. The shape and arrangement of the iron and magnets, the inter-pole angle, and the number of permanent magnets per poles in the rotor side, and the slot opening in the stator side affect the saliency ratio. The saliency ratio is of the factors that influence the flux weakening control. 


\subsubsection{Short-term reliability in PMSM}

The short term reliability is defined as all the operational environment measures that can help to rescue the machine by fast, effective, and optimal fault diagnosis and prognostic systems, and remedial strategies [16-20].

The high power density ratio of rotor to armature and the usage of permanent magnets in a PMSM make this machine quite sensitive to internal short-circuit faults and less reliable than induction, switch reluctance, and somewhat the wound rotor synchronous machines[29].

The internal faults of the machine are of the special issues that seriously treat the reliability of machine-drive systems from the machine side. Therefore any prognostic, prevention, or remedial action that can mitigate the influence internal fault of the machine can improve the availability and maintainability of machine-drive system[30]. Related to faults, it is recognized that the internal faults are a contagious phenomena and can rapidly grow up or mutate from a small defect to an uncompensated tragedy. So, fast and accurate detection, diagnosis, and remedy of fault at its initial states can firstly give a clear indication of incipient failure modes and secondly it greatly simplifies the fault detection, localization, and remedial actions. In addition to this, improving the reliability of each of the machine and the drive in design stage can enhance the reliability of machine-drive system as a whole. In recent years, there has been a huge interest in developing of remedial actions mostly in safety critical applications of machine drive systems. The investigations mainly were focused on equipping or modification of the drive control algorithm with additional fault state controls that could improves the reliability, safety, and security margins of machine[16]. Nevertheless, based upon a survey on the current state of the art in fault tolerant researches of machine-drive systems over the last 30 years in those 
investigations which have proved successful advantage, it is identified that areas of research still require attention to advance the subject[7], [8], [16], [30-37]. Based upon current literatures on fault tolerant area there are still three general open problems: only on-line techniques for rotating machines are studied; specific problems of variable speed drives are not effectively studied; emerging brushless, reluctance and permanent magnet machines of unusual topology are all concentrated upon one category as PM machines.

On the other hand, it is evident that diagnosis of any disease at its initial stages will increase the patient chance to be cured. Therefore, the most effective remedial actions are strongly dependent on a fast and accurate fault detection and diagnosis system and also on the physical characteristics of the machine[38]. It is evident that, the physical characteristics of the machine are specified in the design stage. No effective research effort on the short term reliability joint with design of machines can be found in literature. Machines not only require a reliable fault detection method, but also require a proper remedial action that can maintain the machine-drive system or the connected inverter to continue uninterrupted operation. The most desirable characteristic of a remedial action is to maintain the drive's uninterrupted operation without any degradation in the performance characteristics of the machine in the presence of a fault. The only way to achieve this is through increased redundancy at the design stage, and maybe only redundancy-based approaches can solve this difficulty. In sensitive industries, conservative designs and redundancy policies are commonly applied in order to improve the fault tolerance of the devices[37]. The goal of conservative design policies is to include mitigation measures in the physical design that would reduce fault occurrences through over-sizing the system's capacity or include redundant measures. The concept of redundancy is that: if a part of the system 
fails, there is an extra or back-up solution that is able to operate in place of the failed part to maintain uninterrupted operation. Although these two approaches are important to increase the fault tolerance of an electric machine-drive system, they greatly increase the cost and complexity of the system. Moreover, redundancy may not be practical for an application that has a severe restriction on the installation space, such as in the case of traction drives in electric or hybrid-electric vehicles. To reduce these complexities and increase the efficiency of the whole system, the design optimization of machine for a minimum redundancy and a maximum fault tolerance maybe a proper solution[37]. However, the obtained solution may not be a unique one. Moreover, fault diagnosis and fault tolerance strategies may be put in place dynamically to detect certain failures from its point of inception so as to prevent it from developing into a significant level. From this point of view, operational dynamic HIL-physics-based optimization of machine, drive and diagnosis algorithm system maybe a solution. It is mentioned that, fault tolerant strategies are based on the concept that a faulty system can maintain its uninterrupted operation with the assistance of a modified topology or control algorithm. To implement such approaches as practical entities, the machine drives should be optimally designed to perform the essential tasks of fault detection, fault isolation, remedial and emergency actions in the best possible way.

All in all, there has been a huge interest for online and offline fault detection, analysis, and diagnosis of electrical machine and devising remedial measures from drive side[39]. However, there are still lots of questions that need to be revealed, such as:

- How we can get the maximum robustness in the design of machine-drive systems with regard to internal faults in the machine? 
- How do the control schema and topology of the drive affect the fault diagnosis and remedial actions? Or how should the control recruit to facilitate the fault diagnosis and remedial actions?

- How are the physical characteristics of the machines and control algorithms of the drives associated with the fault?

- What are the benefits of the design of machine-drive systems in a single combined design process concerning special issues like internal fault of machine?

Based on our literature review on fault diagnosis systems [25-28], [38-64], it is clear that electrical techniques have proven to be powerful tools for fault diagnosis of electrical machines, offering comprehensive condition monitoring signals. The difficulties are broad signal bandwidth, complexity of the spectral analysis, the interpretation of their content and the low signal-to-noise ratio of the resultant signal when a fault is present. This situation is exacerbated on variable speed drives where individual fault frequencies may need to be tracked with the drive speed. Moreover, other global indicators of machine health, such as temperature or vibration give clear signals which can be used as complementary methods alongside electrical signals to improve detection. This type of fault diagnosis, multi-parameter fault diagnosis, gives enormous confidence for remedial actions. Multi-parameter fault diagnosis can still be considered a minimalist approach since the minimum effective number of signals should be compared to achieve the necessary detection[35].

On the other hand, relative to the drive, variable speed drives are being applied in increasing numbers to electrical machines. The key problems that may arise in fault diagnosis of variable speed machines are:

- If the speed of the drive remains constant for substantial periods of time, then spectral analysis of flux, voltage, current, power or vibration can still be done 
provided the results are interpreted for the speed and base frequency when the measurements are made.

- If the speed varies significantly, then non-stationary techniques, such as short time interval spectrograms, wavelets or Wigner-Ville techniques, dependent on the rate of change of speed, need to be used.

- If speed varies under control loop action, then the frequency content of the monitoring signals will be affected by the controller bandwidth. In this case, it is possible for the drive controller to suppress fault harmonics in the terminal quantities. However, it may be possible to extract condition monitoring information from signals derived within the controller.

- When machine is supplied from a variable speed drive, all terminal quantities of the electrical machine will be polluted by drive harmonics, and proper filtering will be essential for obtaining a good signal-to-noise ratio.

\subsection{Variable speed-drive control of PMSM[65]}

In the recent decades, with the increase in the memory and speed of microcontrollers (MPU) the vector control is becoming a common technique for design of controllers. The vector control (or namely field-oriented control) of ac machines was introduced in the late 1960s by Laschke, Hasse, and Leonhard [66]. This technique, allows the quick torque response of ac machines similar to that of $\mathrm{dc}$ machines. The design of variable speed drive controller based vector control is connected to the electrical parameter of the PMSM, output power of the PMSM, the maximum EMF, the maximum speed, the inertia of the machine, and the limitation of drive switches[65]. In addition to these technical parameters, environmental concerns, electromagnetic interference (EMI) impose additional limitations to the design of the variable speed drives. The electrical parameter of the machine is normally provided in 
rotor frame of reference. The electrical parameter of the machine affect of the adjustment of PI controller embedded inside the drive. For example, the saliency ratio, the EMF harmonics, armature inductance, the cogging torque, and dynamic core loss from the PMSM are of the factors that affect the modulation technique, current control PI controller, and speed PI controller in the drive, given a desired response needed to be achieved. Therefore attention to compatibility of the PMSM and the chosen drive is necessary to firstly be able to control the PMSM and afterward control the PMSM-drive in the most efficient way[65], [67]. The existing ideas in the variable speed drive control of PMSM are presented in chapter 3.

\subsection{Sensorless control of PMSM}

The vector control of PMSM requires the knowledge of the rotor position. Usually the rotor position is measured by a shaft encoder, resolver, or Hall sensors [68]. The usage of these sensors depends on the shape of the EMF and it defines the modulation technique. The presence of such sensors increases the cost of the overall drive system. It may also reduce the reliability of PMSM-drive system. In addition to these, the unwanted orientation of such sensors, the limited assembly space and rigid working environment with severe vibration and/or high temperature has made the usage of these sensors under serious limitations. Therefore, a robust and responsive sensorless control schemes plays an essential role in the overall cost and reliability of the system. Due to these facts, there have been huge investigations for the estimation of rotor position and speed, especially in the recent decade[68-79].

\subsection{Conventional approach to the design of 3-phase PMSM-drive systems}

Permanent magnet synchronous machine (PMSM) are gradually applied in a variety of industrial and military applications which require high power density, fast dynamic response, accurate control over wide speed ranges. The conventional design 
of PMSM and the drive is a done separately. It means that the reliability and optimality of each PMSM and the drive is considered separately normally chosen for the worse case[80]. This is to guarantee robustness of machine-drive system as a unified system. This static point of view increase overall computational, economical and even the performance cost of PMSM-drive system. The separate design of PMSM-drive forces the designers to sacrifice some of the objectives either or both in drive or in PMSM. It adds economical cost to the system. More importantly, the separate developed algorithms for control and protection embedded in the drive may not be optimal and/or sensible enough to rescue the PMSM-drive system during the fault conditions. It adds computational and the performance cost to the system. Tracking the optimal and reliable working point in PMSM-drive requires the very accurate information from the PMSM[81]. It also requires certain control algorithms in the drive to accurately target the certain machine operating condition at the right time $[80-106]$.

\subsection{Problem statement}

As mentioned in sections 1.2 to 1.5 the reliability and optimality of the permanent magnet machine is not only related to the machine design procedure, material selection but also it is tightly connected to the operational environment conditions as well as the drive control, diagnosis, and remedial actions strategies. Therefore involvement of the machine physical characteristic which are reflected from the design stage into the control side of the machine is necessary to overcome the worsescenario assumptions, fault diagnosis and sensorless control accuracy limitations in the design and control of these systems.

In order to create a computational design environment which covers the interaction of the machine and drive system in design stage, two scenarios were 
assumed. Firstly, both the electrical machine and the drive system are under software development. Secondly, the electrical machine is under software development and the drive is already available. Although, there have been a vast investigations for the first scenario, the second scenario remains unanswered to some extent in the scientific community.

In order to ensure the short-time reliability and optimality which covers of the interaction of machine and drive system in application stage, the creation of modelbased observers and fault diagnosis system is required. Although there have been a huge investigations in model-based observers and fault diagnosis systems in the recent decades, the accuracy of these systems is still under the preparation of an accurate and computationally light model of the machine and adaptive compensation for machine model variations.

Finally, to break the worst case scenario assumptions, the development of realtime thermal and magnetic monitoring system of the electrical machine is needed. If these systems are a model-based one, these can enable us equip the drive with additional control loop to prevent the permanent magnet demagnetization as well as other thermal limitations under any operational contingencies.

\subsection{Research objective}

In this dissertation the idea of co-design of modern PMSM-drive systems is presented. The term co-design is defined as the simultaneous numerical and analytical development and prototyping of such that system. The term modern indicate the advanced sensorless control algorithm, fault diagnosis, fault prognostic and self healer controllers embedded in the drive. These are designed based upon the physics-based model of the machine during the development of the machine. In fact, the design properties of the under development machine impose its behavior, limitation and 
constraints on the drive design process. It is successively applied until desire response and behaviors is achieved.

The co-design process, if successfully achieved, may leads to the optimal and maximum fault tolerant of PMSM-drive system. Indeed, the co-design of PMSMdrive enables us to design accurate and light observers and controllers that assist in adjusting the operating point dynamically according to the ambient temperature, predicted internal situation of PMSM, and the reference command. In the co-design process include two parts of co-design optimization process, co-design of observer and controller development. The co-design optimization process dealt with the simultaneous hardware in the loop (HIL)-design optimization of PMSM-drive system and the HIL-design optimization of PMSM with an existing drive system. The codesign of observer and controller development goal is to include the drive side with the additional control and detection algorithm from the models that is facilitated from the design stage. This objective can also be inserted in the co-design optimization process. On the other hand, the control and protection algorithm embedded in drive uses the very accurate and efficient model of the machine that machine designer has used for the design. The co-design process allows developing reliable and computationally affordable control and fault detection system. The certain objective in co-design of PMSM-drive is to develop sensible inter-turn fault detection, develop a model-based sensor-less control from high to very low speeds, to assess and control certain PMSM properties like temperature and PM demagnetization from drive side. The developed controllers were verified to properly react to harmonic loading conditions, variable temperature environment, and very low and high speeds. The dynamic prediction of the operating point of PMSM and its critical parts from the developed physics-based models allow an optimal and reliable control of PMSM- 
drive. It also helps to expand the torque-speed profile for an acceptable cost added to the control algorithm.

The long term reliability concerns the effect of PMSM design parameters during an isolated PMSM design process. The short term reliability concerns the effective usage of modern observers and controllers to neutralize the effect of internal electrical fault. These two definitions are connected by indirect connection of the physical property and design parameter of PMSM to modern controllers through the use of physicsbased models. The physics-based models calculated from design process enable us to access the physical properties of the machine during operation. Hence the usage of these models in the controllers and fault diagnosis systems enhance the short-term reliability. In addition, because the physics-based models are indirectly connected to the design properties of the machine, the short-term and long term reliabilities are connected in such a way that the multiplication of these two results in a constant measure.

Conventionally, it is assumed that the reliability and cost of a system have direct relation. The higher reliability, the higher the cost will be and vice versa. It is the optimality of a system that balances the reliability and cost of the system in the most desired way. In the co-design process, the higher attention to the short-term and the long-term reliabilities increase the cost of the overall system. However, making a balance between the short-term and long term reliability could decrease the overall cost of reliability.

\subsection{Original contribution and significance}

Permanent magnet synchronous machine has been considered an isolated and reliable system. The term "isolated" highlights the fact that in current literature a PMSM is designed totally independent from a drive system while in reality it can not 
be driven without a drive system. The employment of PMSM in many applications attracted researchers toward design, control and fault tolerance of PMSM drive systems [37].

In design and optimization of the PMSM the design and control of the machine have been considered as isolated problems. The problems and the effects related to interconnectivity of the machine and drive and the role of ambient temperature on the final design have not been studied before.

In control of the machines, because of the lack of an accurate and computationally light model of the machine, the thermal, magntic and saturation effects are ignored in the design of controllers and observers. This has been led to the design of closed loop observers, limiting the power density of the machine for the worse operating condition and neglecting the effect of ambient temperature on the performance of machine drive system.

The fault diagnosis of PMSM is still an open area. For example, only on-line techniques for rotating machines are studied; specific problems of variable speed drives and harmonic loading conditions are not effectively studied; also demagnetization of permanent magnets in PM machines has not been addressed before.

In this dissertation, a solution for the co-design issue of the electric machine and its drive was completed. A solution for co-design of an existing drive and under development electric machine including a solution for highly sensitive model-based observers for control and fault diagnosis of a known electric machine-drive is presented. From the introduced perspectives, traction drives in a hybrid or an electric vehicle and wind based PM generators are some of the applications benefiting from the developments and the results of this proposed dissertation research. 
In the design stage, a FE-based design optimization technique suitable for up to 5KW and 4000 RPM PM machine drive system is developed. The developed technique benefits from the interconnectivity of MATLAB, commercials finite element softwares, and hardware interface tools. The developed optimization technique is extended for hardware in the loop FE-based design optimization of PMSM drive systems. This includes the dynamic compatibility of under development PM machine and an existing drive. It also allows the realistic calculation of torquespeed profile.

In the modeling stage, a computationally light finite-element based thermo-electric model of PM machine is developed. The model includes the effect of iron saturation, magnet demagnetization, and thermal aspect of the machine.

An inductance calculation technique benefiting from field circuit finite element technique is developed. This system allows calculating the inductance as a function of rotor position, direct and quadrature currents.

The thermal behavior of the machine is monitored by an embedded thermal network calculated from the geometry and material of the PM machine. The thermal model is furthur modified with respect to the inter-turn short circuit faults for simulation studies.

A real-time demagnetization assessment system is developed. This system is fed into a real-time demagnetization controller to increase the reliability of PM machine operating at variable ambient temperature and strong reverse magnetic field. The revision of the design ideas based on the worse case scenarios is engaged with this system to increase the flexibility in the choice of magnet thickness in the design stage of PM machine.

An accurate physics-based open loop observer for sensorless rotor position 
estimation and control for wide speed range and different operational contingencies is developed.

In addition, a physics-based open loop observer for inter-turn fault diagnosis system is developed. It is proven that such that observer accurately detect the fault in variable speed closed loop systems especially at low speeds and harmonic loading conditions.

For control purposes an adaptive filtering technique necessary to filter out the observer outputs for wide speed range is developed.

\subsection{Experimental implementations}

Since the actual prototyping is considered a costly process and since any change in the physical structure, size, or material property and type of the machine under fault or normal condition will pollute the fundamental physical rules of the real machine, the design of the machine and its additional fault diagnosis studies is considered an expensive and time consuming and also case sensitive process. In this dissertation, the co-design optimization process is applied on the equivalent HIL-based model of the machine. This was physicaly created by an AC/DC converter connected to a buck converter. This equivalent hardware model is controlled by a thermo-electric saturation dependent model of the machine. The equivalent hardware model enables us to study the connected machine to real drive topology in the presence of fault. It is shown that the comprehensive identified equivalent hardware in loop (HIL) model of the machine can easily reconstructed when the type of fault or machine changes.

A software package is designed to create the inter-connectivity between the MATLAB, INFOLYTICA, and CONTROL DESK. The main function includes the optimization scheme and geometry revision. It is built in an M-file. This M-file sends the dimention and material information of the design to the MAGNET-INFOLYTICA 
and then receives the inductance, cogging torque, back emf and the flux linkage of the machine. Then these values are fed into look up table and the machine equations are built into the Dspace 1103. After that the current adjustable load is run using the Dspace 1103 model while is is currented to the real drive. The developed software is enabled to automatically repeat this process during the optimization process.

The developed ideas in the sensorless control and real-time inductance estimation were experimentally implemented on a $0.25(\mathrm{KW}), 44 \mathrm{VDC}$ brushless DC PM for very low to medium speeds where the real challenges in the model-based sensorless control algorithm appears. The fault detection algorithm was implemented on a $0.8(\mathrm{KW}), 48$ (VDC) PMSM under a harmonic load, and in presence of dynamic eccentricity.

The demagnetization control technique is implemented inside MATLABSIMULINK. It is facilitated from the solution of the non-linear transient finite element analysis with motion. The MAGNET and THERMNET software package are used for finite element analysis.

\subsection{Thesis organization}

The thesis is organized as follows:

In chapter 2 the existing ideas in the classic design of PM machine are reviewed. In the chapter 3, the physics-based modeling of the machine as a critical tool for realistic design of modern PMSM concerning the saturation, thermal, and permanent magnet (PM) demagnetization is reviewed. In addition, the recreation of the physics-based model of the machine in the presence of the inter-turn short-circuit fault is presented in detail. The circuit parameter calculation is presented in chapter 3 as well. In chapter 4 , the existing vector control ideas in voltage source inverters (VSI) and current

source inverters (CSI) are reviewed. Moreover, a novel real-time flux weakening control theory is presented in detail. In chapter 5, a novel physics-based sensorless 
control scheme for PMSM is presented. In chapter 6, a novel physics-based inter-turn fault diagnosis scheme for permanent magnet synchronous machine is presented. In chapter 7, the simultaneous design of PMSM and the drive, called co-design of PMSM-drive system, is explained, and finally the conclusions and recommendation for the future research are presented in chapters 7 and 8 , respectively. 


\section{Chapter 2 Classic Design of Permanent Magnet Synchronous Machine}

\subsection{Overview}

The general objectives in permanent magnet synchronous machine (PMSM) design includes robust reliability for the worst operating points, certain performance measures, maximum torque/volume, minimum cost [109]. Normally, these objectives are satisfied within the isolated mathematical model of the machine concerning thermal, magnetic, environmental, cost and electrical constraints and limitations. Although, the current design trend has been proven to be efficient in design of reliable and optimal PMSM, the underestimation of the drive role in overall reliability and optimality of the PMSM-drive may lead to miss the meaning of an optimal PMSMdrive system. This has not been a hidden fact up to now. The relation between the speed PI controller gains and the machine inertia to the speed rise time is a simple example of such interconnections.

The existing drive control theme such as flux weakening control, sensorless control are normally created from the average model of the machine by means of the vector control theory [110]. This is because the development of such systems is done after the machine is designed. Although, widely accepted and extremely practical, the connection between the drive controllers and the physical property of the PMSM is normally ignored. Such an idea leads to increased error in sensorless algorithms and the decreased efficiency during the flux weakening activation and also increased error in fault detection systems. All of these items could be a direct consequence of post design of drive control and protection algorithm, without attention to the dynamic performance of the machine material and the electrical, magnetic and thermal parameters and their interconnections to the drive. Fitting the drive with model-based 
control systems and/or model-based detection systems and/or model-based assessment systems with the machine design, will result in overall increase in fault tolerant operation of the machine. This is the case if the employed model of the machine reflects the exact electrical and material dynamics at all operating points.

In addition to these issues, the isolated design of PMSM and the drive force designers to always consider the worst operating point for their designs. The worst operating point is defined as a crucial condition at which the machine still sustains lower levels. This critical assumption defines certain rules for the operation of the machine-drive systems. For example, the defined ambient temperature by designers must not be reached in the operational environments by users. If passed, the reliability of the systems will not be guaranteed. The more robust the operating point, the more costly and the higher the volume of the system will be. Another point of view which is proposed in this dissertation is the dynamic worst operating point. This means that, the drive is equipped with additional controllers to prevent the machine property from damage under any operational environment. For example, the selection of the magnet thickness for the worst magnetic operating point for certain ambient temperature does not guarantee the machine operation if the ambient temperature exceeds from the designer expectation. However if the drive is equipped with the demagnetization control, then we will never pass the operating point under any condition given the controller is properly working. This point of view can be entered as a new objective into the design process that indirectly connects the physical property of the machine and the machine dimensions to the controllers during the design stage.

The initialization of the design is a very critical part. The good initializations of a design for a latter optimization process can lead to overall savings in the design time and cost. In this chapter, the basic concept and methods for design of the PMSM is 
presented. The magnetic design, winding arrangement and the effect of the design variables are discussed in details. The role of the machine design parameters on the performance of the machine is reviewed.

\subsection{Convectional geometries in permanent magnet synchronous machine}

The design of radial flux PMSM include many possibilities of shape in both rotor and stator sides[82], [104] and [111]. In radial flux PMSM, the flux crosses from the rotor to the stator radially. Figure 2.1 shows some possibilities for a four poles PMSM rotor. The traditional ARC shape (a), or ARC shape with parallel edges (b), bounded magnet (c), side parallel bottom flat (d), interior flat (e), interior V-type(f), interior double layer V-type (g), interior U-type with one flat magnet on the top $(\mathrm{H})$, interior half magnet number with flat-type (i), interior half magnet number V-type (j), interior U-type with three flat magnets (k) and spoke or flux concentrated (l). These possibilities have emerged because of manufacturing as well as the technical reasons. Assume in all of the depicted rotors in figure 2.1, the outer radius, stack lengths and magnet volumes are equal. The type (a), (b), (c) and (d) are called surface mounted rotor. These normally have higher power density, higher magnet operating point, lower demagnetization risk and the access to higher speeds than nominal speed is limited. The magnetization direction in the types (a) is normally radial, in (b) and (d) it is parallel to the edges. In the (c) type the magnets are created by magnetizing the rotor after assembling it in the rotor yoke. The manufacturing of the type (b) and (d) is easier. Alternatively, the set (e) to (l) are called the interior permanent magnets because the magnets are buried inside the rotor iron. The (e) to (l) cases are beneficial for high speeds because firstly the magnets are entirely enclosed by the rotor iron and the rotor surface is uniform and secondly they benefit from reluctance torque. The interior types support the usage of a flat magnet which is cheaper. In general, the 
magnets of the interior type with the single layer magnet possess higher operating point and lower demagnetization risk. The (f), (g), (h), (j), (k) and (l) have higher flux concentration than (e) and (i) because the magnet surface area is greater than the rotor surface area. This helps the usage of ferrite magnet materials and has benefit of using rectangular block magnets. The type (i) and (j) have lower power density but higher reluctance torque. In addition these two provide higher reliability because they need two permanent magnets to create torque. Types (g) and (h), have lower magnet operating point and the risk of demagnetization in the lower magnet is high, but higher speed is more achievable. Figure 2.2 show the intersection of all of these geometries.

Figure 2.3 shows different possibilities that a PMSM can have in shape in stator sides. Designs 1 to design 6 are called slotted stator. The slotted machine has higher air gap flux density. Also the large contact area between the winding and the stator, air gap and ferromagnetic material promote the heat transfer. The teeth net radial force and the cogging torque in the designs 1 to design 6 are different because they area of teeth opening is different between these designs. The leakage slot inductance in design 4 and design 5 is higher than design 1, design 2 and design 6 and is lower than design 3 . The usage of wires with higher thickness is easier in design 1 , design 2 and design 3. The slot leakage inductance in design 3 is higher than all others. It diverts some of the magnet flux and cause decrease in the power density of the machine. However, it has smaller net cogging torque and net teeth radial force. The slot fill factor in this type is higher that the designs $1,2,4,5$ and 6. 


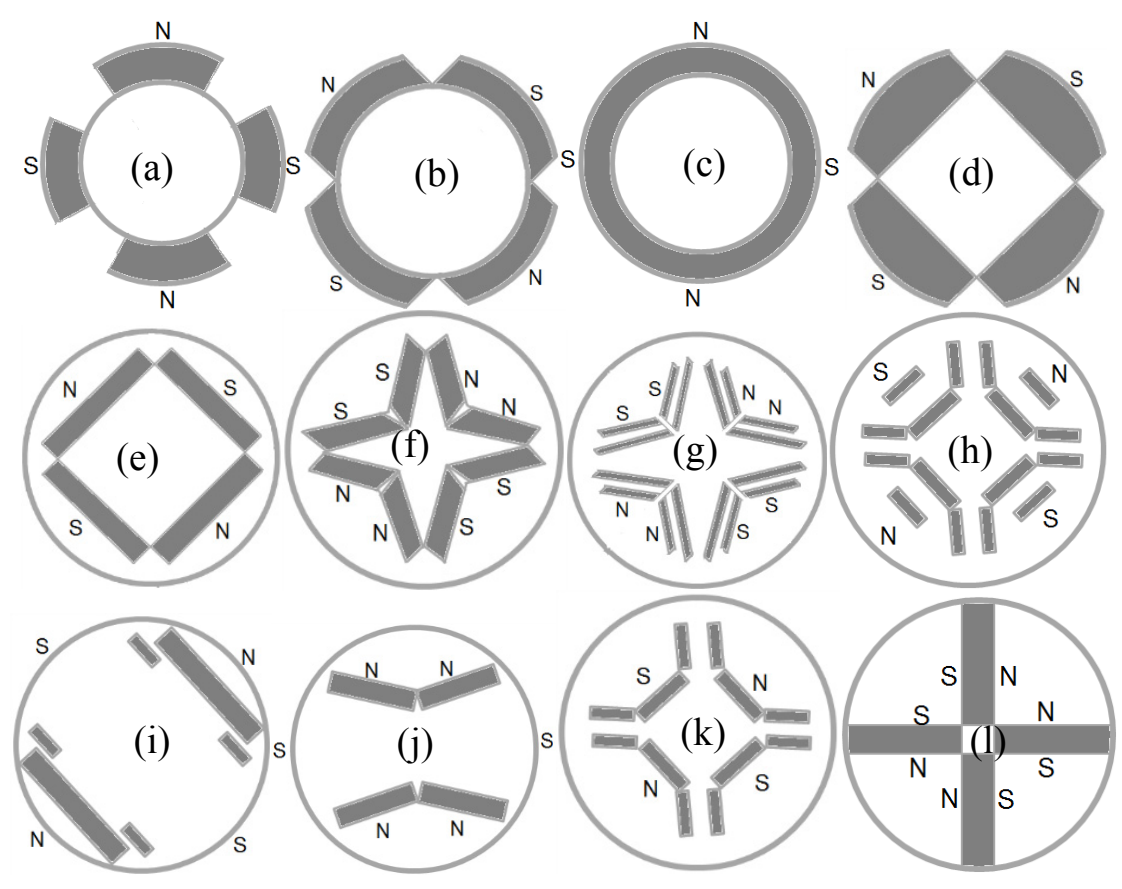

Figure 2.1 Different magnet arrangement in axial flux permanent magnet machine

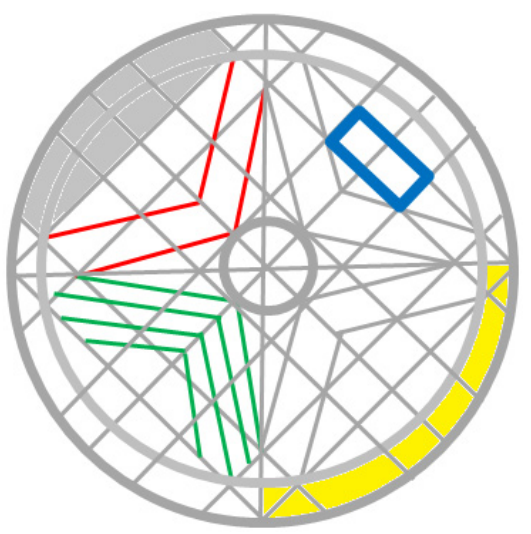

Figure 2.2 Intersection of the conventional geometry for PMSM
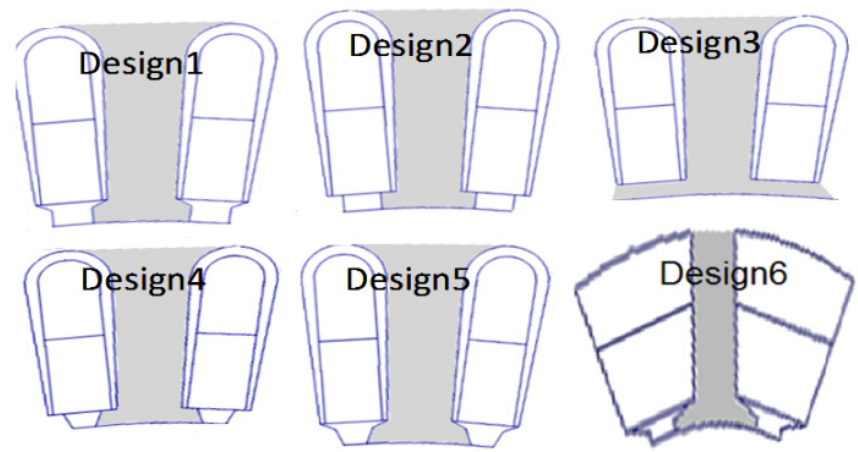

Figure 2.3 Slot opening changes in the $5^{\text {th }}$ test set 


\subsection{Classic design procedure of permanent magnet synchronous machine[82]}

\subsubsection{Permanent magnet circuit model}

In permanent magnet machine, the operating point lies somewhere in the second quadrature, usually at the permeance coefficient of four or more. For design purposes, the permanent magnet model is assumed to be a straight line described by:

$$
B_{m}=B_{r}+\mu_{r} \cdot \mu_{0} \cdot H_{m}
$$

where $H_{m}$ and $B_{m}$ are the operating point. $B_{r}$ is the magnet remanence flux density, $\mu_{r}$ is the magnet recoil permeability. In order to develop the magnetic circuit model for a permanent magnet, a rectangular magnet is assumed; see figure 2.4(a). Then the flux leaving, Figure 2.4(b), the magnet is calculated as:

$$
\begin{aligned}
& \left|B_{m}\right| \cdot A_{m}=\left|B_{r}\right| \cdot A_{m}+\mu_{r} \cdot \mu_{0} \cdot H_{m} \cdot A_{m} \\
& |\phi|=\left|\phi_{r}\right|+\frac{F_{m}}{R_{m}}
\end{aligned}
$$

where $R_{m}$ is the magnetic reluctance of the rectangular permanent magnet. For the arc shape magnet, figure $2.4(\mathrm{c})$, the magnetic reluctance is calculated as:

$$
R_{m}=\frac{\ln \left(1+l_{m} / r_{i}\right)}{\mu_{r} \cdot \mu_{0} \cdot L_{s t} \cdot \theta_{m}}
$$

where $L_{s t}$ is the stack length.

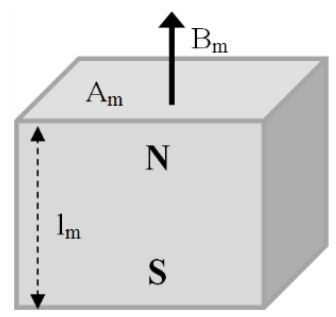

(a)

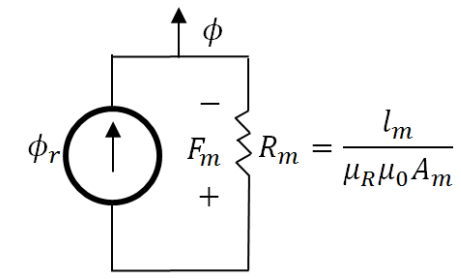

(b)

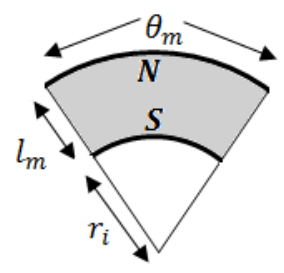

Figure 2.4 Magnetic circuit model of the PM 


\subsubsection{Magnetic circuit of the PMSM with small air gap:}

The idealized open circuit flux of a PMSM is shown in figure 2.5. Assuming infinite permeability for the iron parts, according to the flux path, the equivalent circuit of the machine for one pole pair is defined as figure 2.6. The flux formulation is as follows:

$$
\begin{gathered}
|\phi|=\left|\phi_{r}\right| \times \frac{R_{m}}{R_{m}+\frac{R_{g}}{2}} \\
\phi_{r}=B_{r} \cdot A_{m}=B_{r} \cdot w_{m} \cdot L_{s t} \\
A_{g}=\frac{2 \pi\left(r_{i}+l_{m}\right) \times L_{s t}}{P}
\end{gathered}
$$

where $\mathrm{P}$ is the number of the poles and:

$$
\begin{aligned}
& R_{g}=\frac{g}{\mu_{0} \cdot A_{g}} \\
& R_{m}=\frac{l_{m}}{\mu_{0} \cdot \mu_{r} A_{m}}
\end{aligned}
$$

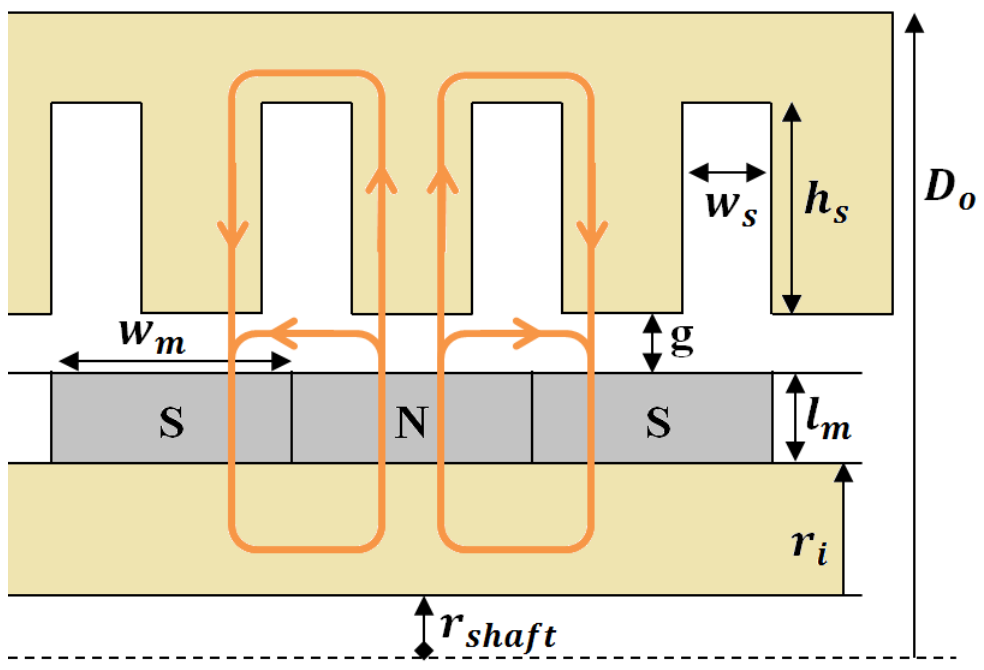

Figure 2.5 Simple geometry and flux path of the surface mount PMSM 


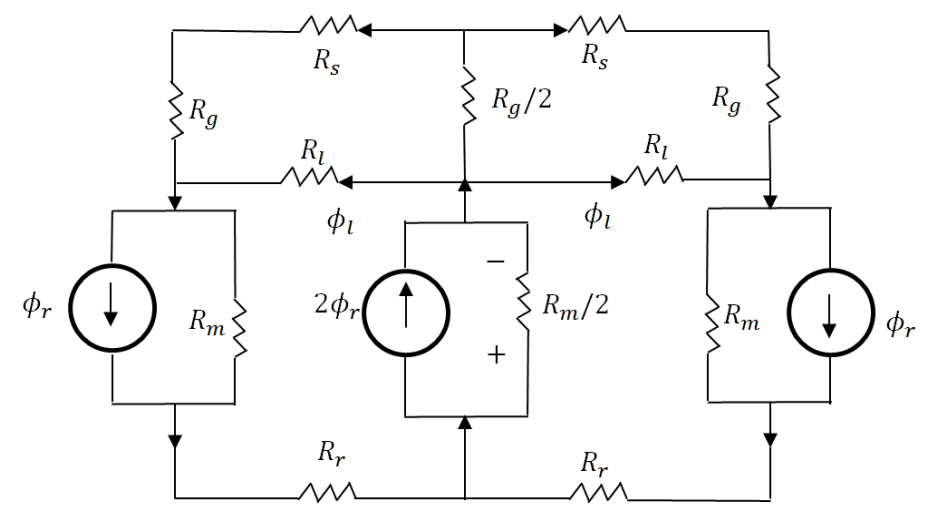

Figure 2.6 Reluctance network of the flux path shown in Figure 2.5

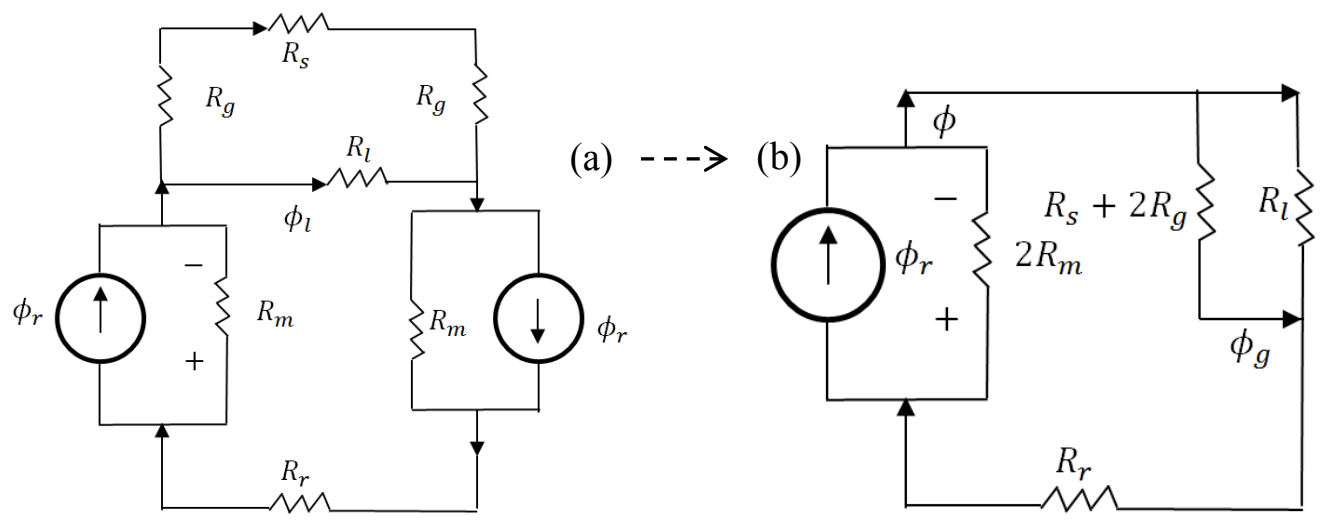

Figure 2.7 Simplified reluctance network of Figure 6

By simplification of the reluctance network shown in figure 2.6, we have:

$$
\begin{aligned}
& |\varphi|=\left|\varphi_{r}\right| \times \frac{2 R_{m}}{\left(\left(R_{S}+2 R_{g}\right) \| R_{l}\right)+R_{r}+2 R_{m}} \\
& \left|\phi_{g}\right|=|\phi| \times \frac{R_{l}}{R_{S}+2 R_{g}+R_{l}}
\end{aligned}
$$

11)

If we assume that $R_{S} \approx R_{r} \gg R_{g}$ and $R_{l}=K_{l} \cdot R_{g}$, for $K_{l} \gg>1$ the equation (210) is simplified as: 


$$
\begin{aligned}
& |\phi|=\left|\phi_{r}\right| \times \frac{2 R_{m}}{\frac{2 K_{l} \cdot R_{g}}{2+K_{l}}+2 R_{m}} \\
& \left|\phi_{g}\right|=|\phi| \times \frac{K_{l}}{2+K_{l}}
\end{aligned}
$$

For the geometry mentioned in figure 2.5, simply in term of the geometrical dimension, the leakage of flux in term of $K_{l}$ is defined as:

$$
K_{l}=\frac{R_{l}}{R_{g}}=\frac{\frac{1}{\mu_{0}} \times\left(w_{m} / g \cdot L_{s t}\right)}{\frac{1}{\mu_{0}} \times\left(g / P \cdot w_{m} \cdot L_{s t}\right)}=\frac{P \cdot w_{m}^{2}}{g^{2}}
$$

where $w_{m}$ is the wide of a pole. Using equation (2-13), the air gap flux density is calculated as:

$$
\left|B_{g}\right|=\frac{\left|\phi_{g}\right|}{A_{g}}=\frac{2 P \cdot\left|\phi_{g}\right|}{\left(2 \pi \cdot L_{s t} \cdot\left(\frac{g}{2}+l_{m}+r_{i}+r_{\text {shaft }}\right)\right)}
$$

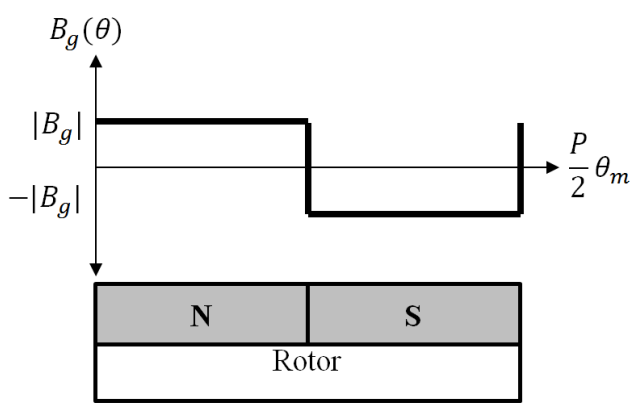

Figure 2.8 Visual illustration of the air gap flux density with respect to the magnets

Mathematically, the magnet magnetization, figure 2.9 , can be of any arbitrary shape defined by a Fourier series. In practice, the magnets are typically magnetized to approximate either radial magnetization or parallel magnetization in addition to the 
radial sinusoidal amplitude magnetization or constant amplitude sinusoidal direction magnetization. The actual magnetization achieved is a strong function of the magnetization fixture and equipment used to magnetize the magnets. To a lesser extent, some magnet materials have a preferred magnetization direction that favors one magnetization over another.

The magnetization vector of a permanent magnet in rotor stationary frame is represented along the radial and tangential direction. The magnetization in polar coordinate is written as:

$$
\vec{M}=M_{r} \cdot \vec{i}_{r}+M_{\theta} \cdot \vec{i}_{\theta}
$$

The radial and tangential magnetizations can be described by Fourier series as:

$$
\begin{aligned}
& M_{r}=\frac{B_{r}}{\mu_{0}} \sum_{-\infty}^{\infty} K_{r n} \cdot e_{p}^{\frac{j n P \theta}{2}} \\
& M_{\theta}=\frac{B_{r}}{\mu_{0}} \sum_{-\infty}^{\infty} K_{\theta n} \cdot e_{p}^{\frac{j n P \theta}{2}}
\end{aligned}
$$

For the radial magnetization the coefficients are calculated as:

$$
\begin{aligned}
& K_{r n}=\left\{\begin{array}{lll}
\alpha_{m} \cdot \sin c\left(\frac{n \alpha_{m} \pi}{2}\right) & n & \text { Odd } \\
0 & \text { Otherwise }
\end{array}\right. \\
& K_{\theta n}=0
\end{aligned}
$$

For the parallel magnetization the coefficients are calculated as:

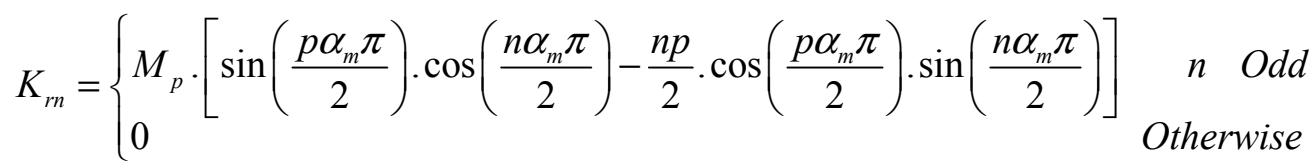




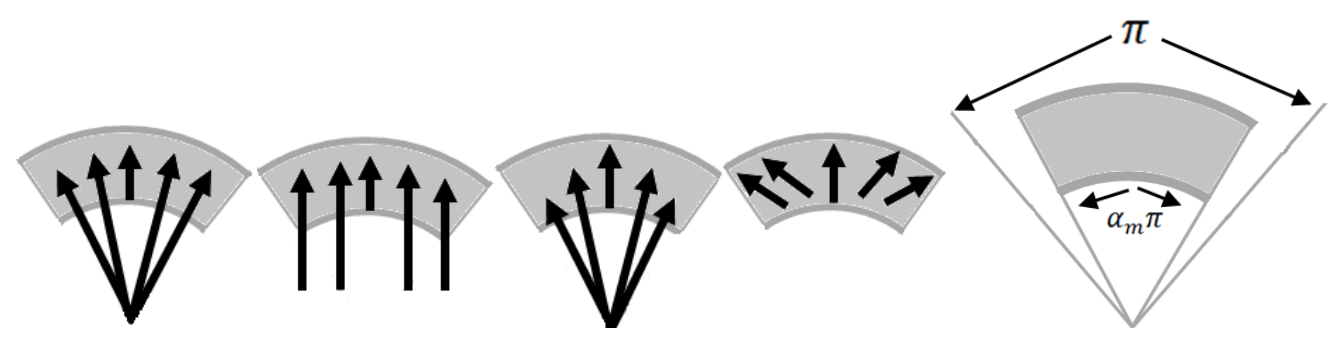

Figure 2.9 The possible demagnetization profiles

$$
\begin{aligned}
& K_{\theta n}=\left\{\begin{array}{l}
-j M_{p} \cdot\left[\cos \left(\frac{p \alpha_{m} \pi}{2}\right) \cdot \sin \left(\frac{n \alpha_{m} \pi}{2}\right)-\frac{n p}{2} \cdot \sin \left(\frac{p \alpha_{m} \pi}{2}\right) \cdot \cos \left(\frac{n \alpha_{m} \pi}{2}\right)\right] n \text { Odd } \\
0 \quad \text { Otherwise }
\end{array}\right. \\
& M_{p}=\frac{p}{\pi\left[1-\left(\frac{n p}{2}\right)^{2}\right]}, \frac{n p}{2} \neq 1
\end{aligned}
$$

When $n p=2$, the fundamental harmonics are:

$$
\begin{aligned}
& K_{r 1}=\frac{\frac{\alpha_{m} \pi}{2}+\cos \left(\frac{\alpha_{m} \pi}{2}\right) \cdot \sin \left(\frac{\alpha_{m} \pi}{2}\right)}{\pi} \\
& K_{\theta 1}=j \frac{\frac{\alpha_{m} \pi}{2}-\cos \left(\frac{\alpha_{m} \pi}{2}\right) \cdot \sin \left(\frac{\alpha_{m} \pi}{2}\right)}{\pi}
\end{aligned}
$$

For the Radial Sinusoidal amplitude magnetization the coefficients are calculated as:

$$
\begin{array}{ll}
K_{r n}= \begin{cases}\frac{1}{2} & n= \pm 1 \\
0 & \text { Otherwise }\end{cases} \\
K_{\theta n}=0
\end{array}
$$

For the radial angle magnetization and ideal Halbach array are similar. The coefficients are calculated as: 


$$
\begin{gathered}
K_{r n}=\left\{\begin{array}{lc}
\frac{1}{2} & n= \pm 1 \\
0 & \text { Otherwise }
\end{array}\right. \\
K_{\theta n}= \begin{cases}\frac{j^{n}}{2} & n= \pm 1 \\
0 & \text { Otherwise }\end{cases}
\end{gathered}
$$

\subsubsection{Effect of temperature on the magnet remnance flux density:}

In general, for life assessment studies of PM materials, the relation of temperature and PM loss can be categorized in three classes. These are reversible losses, irreversible but recoverable losses and irreversible and unrecoverable losses[104]. The reversible loss is defined for all the operating points above the knee point. It is the loss that is recovered when the magnet returns to its original temperature. The effect of temperature on the remnance $B_{r}$ is described as:

$$
B_{r}\left(T_{1}\right)=B_{r}\left(T_{0}\right) \cdot\left[1+\Delta_{B}\left(T-T_{0}\right)\right]
$$

where $T$ is the magnet temperature, $T_{0}$ is reference temperature and the $\Delta_{B}$ is the reversible temperature coefficient.

In order to include the thermal effects, the thermal network of the machine is required. The thermal aspects also can be taken into account by limiting the current density. Here in the classic design, the thermal aspect is assumed to be managed by the current density, however the transient thermal model appear in the optimization process. 


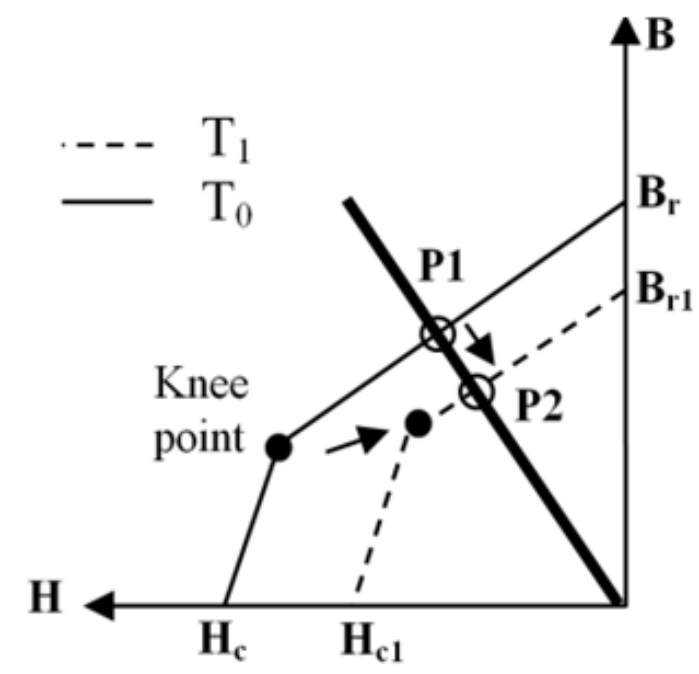

Figure 2.10 The effect of the temperature on the permanent magnets

\subsection{Winding arrangement for the radial flux PMSM[82]}

One phase winding for all magnet poles does not able to produce torque for all rotor positions. This is because every 180 degree, the EMF falls to zero and as a result the output power reaches zero. In order to resolve this issue, the PMSM have more than one phase winding. Most of the PMSM machines have 3-phase winding. The phase winding orientation is set to cover the zero crossing point uniformly. The higher number of the phase winding, the higher flexibility for EMF zero coverage will be. This is very important for fault tolerant PMSM machines. However, the higher number of the phases, the higher number of power electronic switches in the drive would be. Therefore it is uncommon to see machine having more than three phase winding. However, when the power level goes high, it makes sense to use more than three phases. The winding arrangement has serious impact on the flux linkage of the machine. The most commonly used windings for three-phase radial-field permanent magnet synchronous machines are the overlapping concentrated, overlapping distributed, non-overlapping concentrated with all teeth wound and non-overlapping concentrated alternate teeth wound, see figure 2.11. 
The comparison between the distributed and the concentrated windings show that the typical copper slot fill factor in distributed windings is about $34 \%$ to $45 \%$ where in the concentrated windings it is higher around $50 \%$ to $65 \%$. In addition the stator structure in the distributed winding should be continuous lamination where in the distributed windings it can be continuous or segmented. The distributed overlapping winding generally results in a more sinusoidal magneto-motive force (MMF) distribution and EMF waveform.
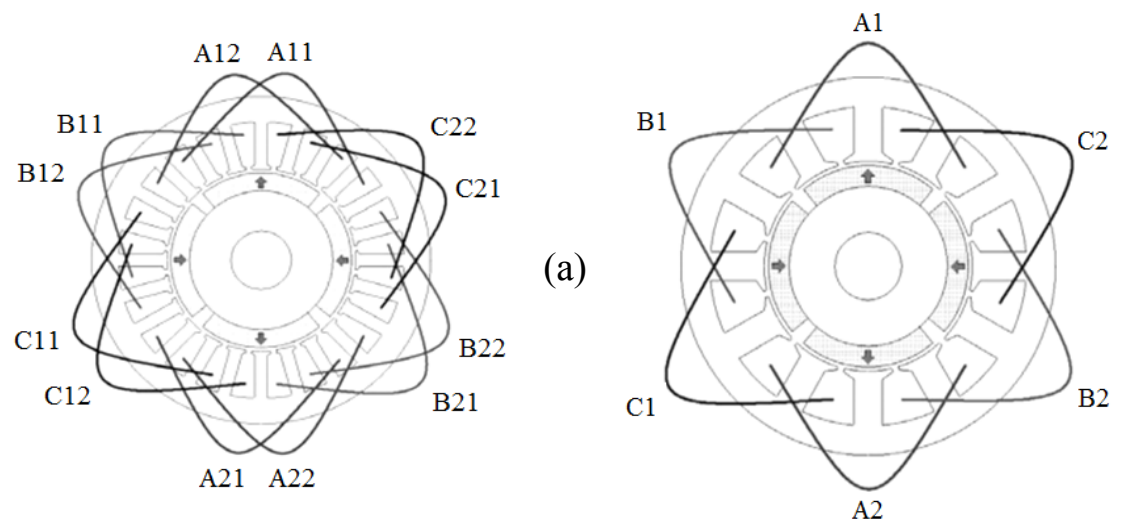

(b)

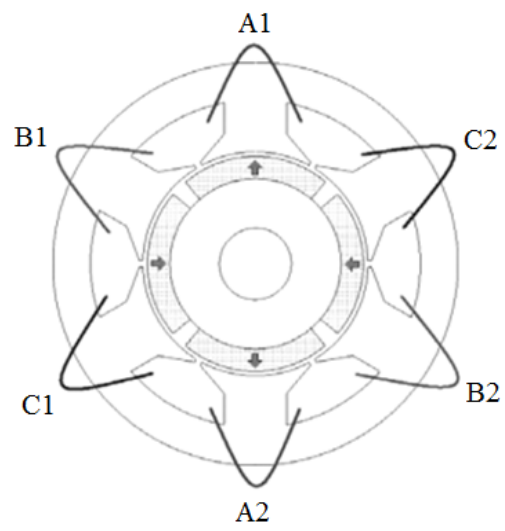

(c)

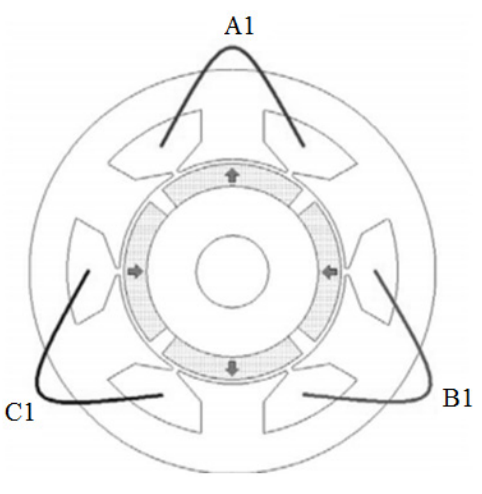

(d)

Figure 2.11 The overlapping distributed winding (a), overlapping concentrated (b), non-overlapping concentrated double layer (c), non-overlapping concentrated single layer (d) [100]

Fault tolerance is one of the key issues with PM machines in general, particularly in safety-critical applications. The main reason is that the PMs cannot be de-excited in 
case of a fault particularly for a generator that is coupled to a prime mover. The nonoverlapping provide many advantages in terms of fault tolerance particularly single layer windings. Single layer windings provide very low mutual coupling between the various phases as well as physical separation.

The comparison of the, single layer and double layer non-overlapping winding show that the mutual phase coupling in the double layer is higher than single layer through significant slot leakage. The single layer has very low mutual coupling and it is recommended for fault tolerant machines. The end turns in the double layer are shorter. The phase inductance in the double layer winding is lower than the phase inductance in single layer winding. The single layer winding offer higher phase leakage inductance. The rotor and magnet losses in the double layer winding are lower. This is due to the lower fundamental stator space harmonics. The flexibility in the slot/pole combination in the double layer winding is higher than that of the single layer winding. The single layer winding is easier in manufacturing since just one coil side fill each slot. The back EMF in the double layer winding can be more similar to sinusoidal waveform.

The concentrated and non-overlapped winding have shorter winding and regions, short manufacture time and are more stable against turn-turn fault. However, they do not have enough flexibility for shaping the back EMF. Because of the requirement, in certain number of slots/ pole/phase, the control of cogging torque through the selection of the proper number of slots/pole is limited. Alternatively, the distributed and non-overlapping windings provide the same fault resistive performance and because of the special distribution of the winding the control of the shape of the back EMF is easier. The concentrated and non-overlapped winding has several advantages. This is mainly due to several advantages that this type of windings provides. These 
include high-power density, high efficiency, short end turns and high slot fill factor. In particular the case when coupled with segmented stator structures, low cogging torque, flux-weakening capability and fault tolerance.

Compared to overlapping windings, the non-overlapping winding has a shorter end-winding overhang, a simplified winding insulation and a reduced number of stator coils [10]. This implies that the active length can be increased, reduced copper in the end windings, an improved efficiency and reduced manufacturing costs. The properties are summarized as follows:

A non-overlapping double layer concentrated winding has a coil wound around each tooth and the coil pitch is fixed. This usually leads to the use of round wire for which the insulation class 200 is not typical. The number of coils is equal to the number of slots. To reduce the cogging torque any further, the rotor must be skewed Non-overlapping single layer. Here, only every second stator tooth has a coil wound around it and as a consequence, the coil pitch can be varied to improve the motor performance. This then leads to a stator design with alternating tooth widths similar to form-wound coils and insulation class 200 can be used. The coil number is half the slot number[100]. For the same slot dimensions, a higher copper fill factor means less copper loss. It is not necessary to skew the rotor since the stator slot pitch can be varied to reduce the cogging torque. In general, a disadvantage of non-overlapping concentrated windings, compared to distributed windings is the higher magnetomotive force harmonics in the air gap[102]. Due to the higher order harmonics, the increased leakage inductance causes higher core losses and a need for a higher inverter rating. The field-weakening current in the constant power region will however be lower[80]. 


\subsubsection{Valid number of slots for specific number of magnetic poles for double layer winding}

The PMSM can have $P$ magnet poles and $N_{S}$ number of slots. From this infinite set, only a few sets satisfy the maximum use of the stator slot and lead efficient torque production[82]. For this purpose an initial assumption is required to find the valid number of slots and poles. We then initiate the algorithm for finding the winding arrangement. As an example:

- The machine has three phases.

- There are two coil sides in each slot

- Only balanced winding are considered

- The number of the slots/pole/phase is considered to be less than two.

- All coils have the same number of turns and all coil spans are equal.

- The number of slots is higher than the number of poles.

- Number of slots/ pole/ phase is common to denote as $N_{s p p}$. When $N_{s p p}$ is larger than or equal to 1 the winding is called distributed. Distributed windings can be divided into integer ( $N_{s p p}$ an integer) and fractional ( $N_{s p p}$ a fraction) windings. Machines is designed with concentrated windings where $N_{s p p} \leq 1$.

- The number of poles must be an even number.

It is evident that only certain combination of magnet poles and stator slots fit the preceding winding assumption. At first, for a three phase motor, the number of slots is necessary to be a multiple of three. Secondly, each of the three phase winding must produce a back EMF of the same amplitude and shape as well as shifted by 120 electrical degrees. These two situations define balanced windings. The number of slots per pole per phase is defined as: 


$$
N_{s p p}=3 N_{s} / P
$$

Based on this definition, if $N_{s p p}$ become an integer then it is called the "integral slot motor" and if $N_{s p p}$ has a fractional part it is called "fractional slot motor". In an integral slot motor, the back EMF of all coils making up a phase winding EMF are in phase even if we have a fractional coil span. Therefore we can make different arrangement of series and parallel coils. In fractional slot winding, the the back EMF of coils is not in phase, therefore the net back EMF has a different shape and amplitude. In fractional slot machine, all coils in a phase should be in series.

Mathematically, the phase offset can be determined by identifying the electrical angle of each slot relative to zero. Since the mechanical angle of a slot is $\theta_{s}=360 N_{s}$ the electrical angle of the $k^{\text {th }}$ slot is defined as:

$$
\theta_{\text {select. }}(k)=k \times \frac{P}{2} \times \frac{360}{N_{S}}=k \times P \times \frac{180}{N_{S}}
$$

For a 3-phase machine the angle between phases is equal to 120 degree, therefore for any values of $K, P$ and $N_{s}$ that the below equation should be satisfied if $K_{0}$ is the phase offset.

$$
\operatorname{rem}\left(\mathrm{K}_{0} \times \mathrm{P} \times \frac{180}{\mathrm{~N}_{\mathrm{s}}}, 360^{\circ}(\text { Electrical })\right)=120
$$

\subsubsection{Valid coil span concerning number of magnetic poles}

After choosing of a valid number of slots and phase offset, the next step toward winding arrangement is to choose the coil span[82]. The angular slot pitch, $\left(P \times 180 / N_{s}\right)$, is the electrical phase shift between two adjacent slot and therefore the nominal coil span is defined by the defining the $N_{S} / P$. However, this may be a 
fractional number or a number less than one. The coil span can be readily calculated as:

$$
S^{*}=\max \left[\operatorname{fix}\left(N_{s} / P\right), 1\right]
$$

where $\max [a, 1]$ return the maximum value of $" a "$ and one. And the function $\operatorname{fix}\left(N_{s} / P\right)$ returns the integer portion of its argument.

\subsubsection{Number of coils per phase for double layer windings}

The Number of coils per phase can be calculated after the number of slots is specified, it is calculated as:

$$
N_{c p h}=\frac{N_{s}}{3}
$$

\subsubsection{Consideration in the design of non-overlapping windings}

In double layer non-overlapping winding, the number of coils/pole/phase is equal

to $N_{s p p}$. In the single layer non-overlapping windings, it is equal to $\frac{N_{s p p}}{2}$, see figure 2.11(d). In the non-overlapping windings, the coil span is always one slot. In double layer windings, therefore the coil span is equal to $K_{0}$. In the single layer windings, it is $K_{0}+2$ [82].

\subsubsection{Winding arrangement $[82]$}

The winding arrangement possibilities is done for selected number of slots, phase offset and coil pitch. The effort in finding the sets of $\left[P, N_{s}, S^{*}, K_{0}\right]$ is created by iteration of equations (2-33) and (2-34) for a limited number of slots and magnet poles. Following the selection of all potential number of slots, coil pitch and phase offset by the methods described above, it is time to create a proper winding arrangement. This means for example if the first coil side in phase A is started from 
the slot number one, the other side of this coil is placed in slot $S^{*}+1$. The phase off set, $K_{0}$, determines the number of slots in clockwise direction for putting the first coil side in the phase "b". The second side of the coil B is placed in slot number $K_{0}+S^{*}$. In addition, if the number of coils per pole i.e. $N_{s} /(3 \times P)$ be larger than one the winding is distributed winding. In this case, following the placement of the first coil in phase $\mathrm{A}$, the second coil of this phase which has the same coil span can be placed in $S^{*}-1$ possible location. Similarly, the third coil can be placed in the $S^{*}-2$ location. Therefore we can say that in the double layer distributed windings, $S^{*} \geq 2$ and $N_{s p p}>1$. There is $S^{*} \times\left(S^{*}-i\right) \times \ldots \times\left(S^{*}-N_{c p}\right)$ options for winding arrangement. Where $N_{c p}$ is the number of coil per poles i.e. $N_{s} /(3 \times P)$ or the $N_{s p p}$. For $S^{*}=1$ and $N_{s p p}<1$, there is only one feasible possibility for winding arrangement. In this case, the windings are the concentrated overlapping or non-overlapping. In this situation, the winding linkage area is determined for one slot pitch angle, i.e. $\left(P \times 180 / N_{s}\right)$. It is illustrated in figures 2.12.a and 2.12.b for two different slot pitch angles. This figure is for non-overlapping windings; however, the concentrated-overlapping winding has similar behavior. Again, the number of coils is equal to $N_{s} / 3$ in the concentrated windings and each coil fill half of the slot in the double layer windings.

\subsubsection{Winding linkage area}

For designing a machine with concentrated or distributed windings, one would seek to achieve the highest possible winding factor and therefore the coil spans are better to be chosen equally. If the coil span is not chosen equally nor has space shift, normally in distributed windings, the winding can have a significant role in shaping 
the flux linkage and back EMF. To express the role of the winding arrangement, many authors have expressed the concept of the winding factor. In fact, the winding factor for a specific winding expresses the ratio of the flux linked by that winding compared to the flux that would have been linked by a single-layer full-pitch non-skewed integer-slot winding with the same number of turns and one single slot per pole per phase. The torque of an electric motor is proportional to the fundamental winding factor. An alternative way for the usage of the winding factor is the usage of the direct Fourier series. The winding special distribution or coil span or the winding axial shift (skew) affects the flux linkage of the machine and therefore the performance of the machine. The winding factors define a simple way to seek these influences. Here, the concept of the winding linkage area, which is equivalent to the winding factor, is defined. The winding linkage area for the concentrated windings is defined as:

$$
W_{L A}(\theta)= \begin{cases}0 & \theta_{\text {electrical }}<0 \\ 0 & 0<\theta_{\text {electrical }}<\frac{180 \times\left(N_{S}-P\right)}{2 \times N_{S}} \\ N_{W} & \frac{180 \times\left(N_{S}-P\right)}{2 \times N_{S}}<\theta_{\text {electrical }}<180-\frac{180 \times\left(N_{S}-P\right)}{2 \times N_{S}} \\ 0 & 180-\frac{180 \times\left(N_{S}-P\right)}{2 \times N_{S}}<\theta_{\text {electrical }}<180 \\ 0 & 180<\theta_{\text {electrical }}<180+\frac{180 \times\left(N_{S}-P\right)}{2 \times N_{S}} \\ N_{w} & 180+\frac{180 \times\left(N_{S}-P\right)}{2 \times N_{S}}<\theta_{\text {electrical }}<360-\frac{180 \times\left(N_{S}-P\right)}{2 \times N_{S}} \\ 0 & 360-\frac{180 \times\left(N_{S}-P\right)}{2 \times N_{S}}<\theta_{\text {electrical }}<360 \\ 0 & \theta_{\text {electrical }}>360\end{cases}
$$

For the distributed windings based on the possibilities of the winding arrangement, $S^{*} \times\left(S^{*}-1\right) \times \ldots \times\left(S^{*}-N_{c p}\right)$, the winding linkage change, see figure 
2.13. It general for the first coil, it can be defined as equation (2-37). If the second coil be in the same slot as the first coil, the wilding linkage is the same as the first coil's winding linkage. If it is shifted to the right by one slot, the winding linkage area is defined as equation $(2-38)$. If the $(i+1)^{t h}$ coil is shifted in the slot to the right, clockwise, the winding linkage area is defined as equation (2.39). In the distributed windings the overall winding linkage area is the summation of all coil's single linkage area which is defined in equation (2-40). In equation (2-40), the number of coils per pole is $N_{c p}$ and $N_{W}$ is the number of the winding turns per pole.

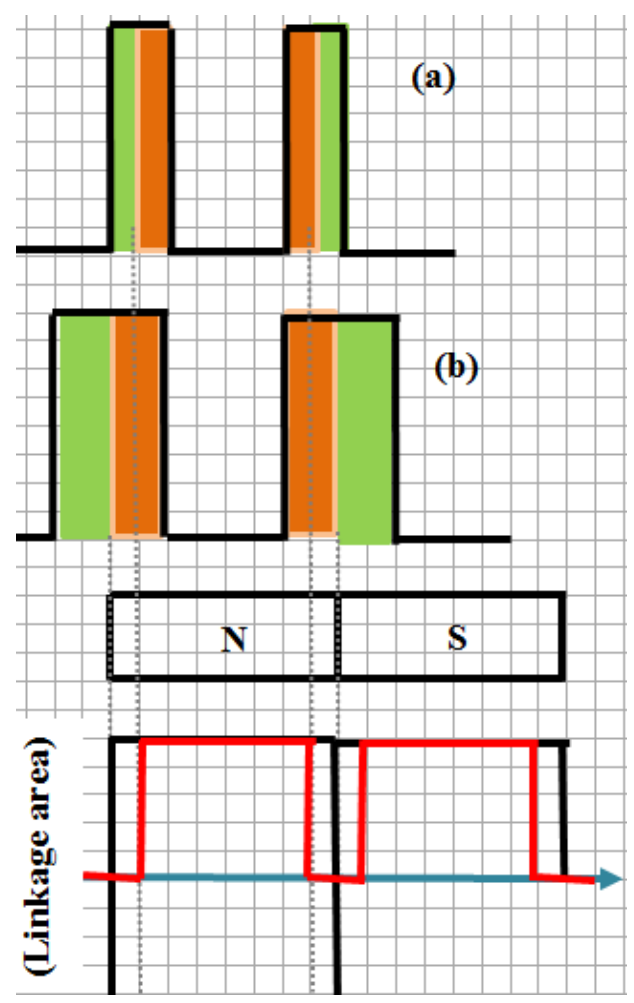

Figure 2.12 Winding linkage area of a coil for the concentrated winding with different slot pitch 
$W_{L A 1}=$

$$
\begin{aligned}
& 0 \quad 0<\theta_{\text {electrical }}<\frac{180 \times\left(N_{S}-P \times S^{*}\right)}{2 \times N_{S}} \\
& \frac{N_{w}}{N_{c p}} \quad \frac{180 \times\left(N_{S}-P \times S^{*}\right)}{2 \times N_{S}}<\theta_{\text {electrical }}<180-\frac{180 \times\left(N_{S}-P \times S^{*}\right)}{2 \times N_{S}} \\
& 0 \quad 180-\frac{180 \times\left(N_{S}-P \times S^{*}\right)}{2 \times N_{S}}<\theta_{\text {electrical }}<180 \\
& \left\{0 \quad 180<\theta_{\text {electrical }}<180+\frac{180 \times\left(N_{S}-P \times S^{*}\right)}{2 \times N_{S}}\right. \\
& \frac{N_{w}}{N_{c p}} \quad 180+\frac{180 \times\left(N_{S}-P \times S^{*}\right)}{2 \times N_{S}}<\theta_{\text {electrical }}<360-\frac{180 \times\left(N_{S}-P \times S^{*}\right)}{2 \times N_{S}} \\
& \mid \begin{array}{ll}
0 & 360-\frac{180 \times\left(N_{S}-P \times S^{*}\right)}{2 \times N_{S}}<\theta_{\text {electrical }}<360 \\
0 & \theta_{\text {electrical }}>360
\end{array} \\
& { }^{W A 2}=
\end{aligned}
$$

$$
\left\{\begin{array}{l}
0 \quad \frac{180 \times P}{N_{S}}<\theta_{\text {electrical }}<\frac{180 \times\left(N_{S}-P \times S^{*}\right)}{2 \times N_{S}}+\frac{180 \times P}{N_{S}} \\
\frac{N_{w}}{N_{c p}} \frac{180 \times\left(N_{S}-P \times S^{*}\right)}{2 \times N_{S}}+\frac{180 \times P}{N_{S}}<\theta_{\text {electrical }}<180-\frac{180 \times\left(N_{S}-P \times S^{*}\right)}{2 \times N_{S}}+\frac{180 \times P}{N_{S}} \\
0 \quad 180-\frac{180 \times\left(N_{S}-P \times S^{*}\right)}{2 \times N_{S}}+\frac{180 \times P}{N_{S}}<\theta_{\text {electrical }}<180+\frac{180 \times P}{N_{S}} \\
0 \quad 180+\frac{180 \times P}{N_{S}}<\theta_{\text {electrical }}<180+\frac{180 \times\left(N_{S}-P \times S^{*}\right)}{2 \times N_{S}}+\frac{180 \times P}{N_{S}} \\
\frac{N_{w}}{N_{c p}} 180+\frac{180 \times\left(N_{S}-P \times S^{*}\right)}{2 \times N_{S}}+\frac{180 \times P}{N_{S}}<\theta_{\text {electrical }}<360-\frac{180 \times\left(N_{S}-P \times S^{*}\right)}{2 \times N_{S}}+\frac{180 \times P}{N_{S}} \\
0 \quad 360-\frac{180 \times\left(N_{S}-P \times S^{*}\right)}{2 \times N_{S}}+\frac{180 \times P}{N_{S}}<\theta_{\text {electrical }}<360+\frac{180 \times P}{N_{S}} \\
0 \quad \theta_{\text {electrical }}>360+\frac{180 \times P}{N_{S}}
\end{array}\right.
$$


$W_{L A i}=$

$\left\{\begin{array}{l}0 \quad \frac{180 \times P \times i}{N_{S}}<\theta_{\text {electrical }}<\frac{180 \times\left(N_{S}-P \times S^{*}\right)}{2 \times N_{S}}+\frac{180 \times P \times i}{N_{S}} \\ \frac{N_{w}}{N_{c p}} \frac{180 \times\left(N_{S}-P \times S^{*}\right)}{2 \times N_{S}}+\frac{180 \times P \times i}{N_{S}}<\theta_{\text {electrical }}<180-\frac{180 \times\left(N_{S}-P \times S^{*}\right)}{2 \times N_{S}}+\frac{180 \times P \times i}{N_{S}} \\ 0 \quad 180-\frac{180 \times\left(N_{S}-P \times S^{*}\right)}{2 \times N_{S}}+\frac{180 \times P \times i}{N_{S}}<\theta_{\text {electrical }}<180+\frac{180 \times P \times i}{N_{S}} \\ 0 \quad 180+\frac{180 \times P \times i}{N_{S}}<\theta_{\text {electrical }}<180+\frac{180 \times\left(N_{S}-P \times S^{*}\right)}{2 \times N_{S}}+\frac{180 \times P \times i}{N_{S}} \\ \frac{N_{w}}{N_{c p}} 180+\frac{180 \times\left(N_{S}-P \times S^{*}\right)}{2 \times N_{S}}+\frac{180 \times P \times i}{N_{S}}<\theta_{\text {electrical }}<360-\frac{180 \times\left(N_{S}-P \times S^{*}\right)}{2 \times N_{S}}+\frac{180 \times P \times i}{N_{S}} \\ 0 \quad 360-\frac{180 \times\left(N_{S}-P \times S^{*}\right)}{2 \times N_{S}}+\frac{180 \times P \times i}{N_{S}}<\theta_{\text {electrical }}<360+\frac{180 \times P \times i}{N_{S}} \\ 0 \quad \theta_{\text {electrical }}>360+\frac{180 \times P \times i}{N_{S}}\end{array}\right.$

$W_{L A}(\theta)=\sum_{i=1}^{N_{c p}} W_{L A i}(\theta)$

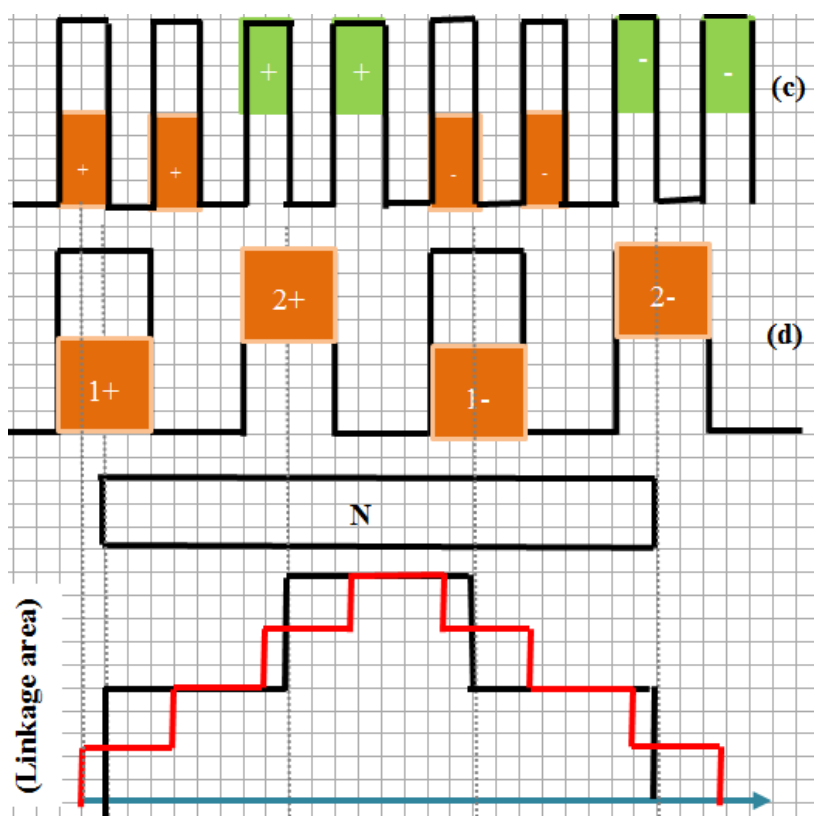

Figure 2.13 Linkage area Non-overlapping winding concentrated and distributed winding with different coil sections 


\subsubsection{Other consideration for selection of the proper winding arrangement}

\subsubsection{Effect of the number of the poles and slots on radial force and cogging torque}

Although the defined rules for selecting the number of poles and slots of the winding arrangement may result in ideal flux linkage in a PMSM, the mathematical relationship between the number of poles and slots to the cogging torque of the machine and to the radial force is a necessary constraint in the overall choice of the number of poles and slots[101]. The net radial force has little impact on the machine performance. However, in low audible noise application, the presence of radial force can limit the motor acoustic performance. On the other hand, the cogging torque which is the result of the stator slot interaction and the rotor magnetic field is the first source of torque ripple. Normally, it is considered as more of a negative impact on the machine performance than the radial force.

In reference [85], the author has derived the relationship between the cogging torque, number of slots and number of poles. The cogging torque tries to align the permanent magnet with the teeth to minimize the air gap reluctance. In order to identify the relationship between the cogging torque and the number of slots and the number of poles, assume that the cogging torque of the slots have the same shape but offset from each other by angular slot pitch angle. Furthermore, the cogging torque is periodic because the south pole creates the same cogging torque in reverse direction. As a result the frequency of the cogging torque is twice as the frequency of the fundamental electrical frequency. As a result, the net cogging torque, $T_{c o g}$, experienced by the all stator tooth can be written as: 


$$
T_{\operatorname{cog}}=\sum_{n=-\infty}^{\infty} T_{n} \times\left\{\sum_{k=0}^{N_{S}-1}\left(e^{-j 2 n \cdot k \cdot \frac{180 \times P}{N_{S}}}\right) e^{j n 2 \theta}\right\}
$$

where $\theta$ is the electrical angle, $k$ is the stator number of teeth number. The $T_{n}$ is the constant harmonic amplitude with the order of $n$.

The per-tooth harmonics that appear in the net cogging torque satisfy the simple relationship:

$$
G C D\left(N_{s}, n, P\right)=N_{s}
$$

where $G C D$, is the greatest common divisor operator.

The equation (2-42) implies that in the integral slot machines, i.e. $G C D\left(N_{s}, P\right)>1$, the cogging torque is higher than in the fractional slot machines. Physically, this is because in fractional slot machine, the portion of the cogging torque resulted from north pole is canceled out by the cogging torque of the south pole. The periodicity of the cogging torque in the integral slot machines is $\left(N_{s} / P\right)$.

In addition to the undesirable tangential force, i.e. the source of cogging torque, a machine may experience an undesirable radial force between the rotor and stator, this force varies as the rotor rotates. In this case, similar to the cogging torque, the net radial force is the summation of all the single radial forces on the stator teeth. The repetition of frequency of the radial force is similar to the cogging torque. Because we are facing a radial flux machine with curved stator teeth, the net radial force is better expressed in the $\mathrm{X}$ and $\mathrm{Y}$ directions separately as[82]: 


$$
\begin{aligned}
& \left.F_{X}=\sum_{n=-\infty}^{\infty} F_{n} \times\left\{\sum_{k=0}^{N_{S}^{S}-1} \cos \left(k \cdot \frac{180 \times P}{N_{S}}\right) \times e^{-j 2 n \cdot k \cdot \frac{180 \times P}{N_{S}}}\right) e^{j n 2 \theta}\right\} \\
& \left.F_{Y}=\sum_{n=-\infty}^{\infty} F_{n} \times\left\{\sum_{\substack{N_{S}-1 \\
k=0}}-\sin \left(k \cdot \frac{180 \times P}{N_{S}}\right) \times e^{-j 2 n \cdot k \cdot \frac{180 \times P}{N_{S}}}\right) e^{j n 2 \theta}\right\}(2
\end{aligned}
$$

where $\theta$ is the electrical angle, $k$ is the stator number of teeth. The $F_{n}$ is the constant harmonic amplitude with the order of $n$.

The harmonic that contribute to the net radial force satisfy:

$$
n=\frac{(2 q-1) N_{s} \pm 1}{P} \quad q \in\{1,3,5, \ldots\}
$$

Based on the equations (2-43-a) and (2-43-b), the rotor experience zero net radial force when $G C D\left(N_{s}, P\right)>1$. Physically, this means that there is a geometrical symmetry in the stator between the stator teeth around the air gap.

As it was explained, to have a lower cogging torque $G C D\left(N_{s}, P\right)<1$ and for having a zero net radial force $G C D\left(N_{s}, P\right)>1$. In addition, since machines with concentrated windings always have fractional type winding, i.e. $G C D\left(N_{s}, P\right)<1$. One should be aware of this fact during the machine design. These sub-harmonic force waves (i.e. force waves with longer wavelength than the pole width) will, if the machine is badly designed, create audible noise and vibrations. The important issue here is that the force wave, with its harmonics, should not excite any of the resonant frequency of the machine. In order to find a common choice between all of the contradiction, the skewing technique is one of the solutions. The skewing of stator slots along the axial direction linearly is common in PMSM with concentrated 
winding. The skewing can be also done in the rotor which is more common in tubular permanent magnet machine. In fractional slot permanent magnet machines, the skewing does not eliminate the radial forces but it produces an axial variation in it and reduces the net amplitude. Also, a linear skew in the stator slot can completely eliminates the net cogging torque if the maximum skew angle is properly selected. However doing so, will also affect the flux linkage and the back EMF of the machine since it acts similar to a low pass filter. This means that the skewing reduces the power density of the machine. In [98], it is proposed that the selection of high number of " $\mathrm{N}_{\mathrm{s}} / \mathrm{P}$ " for minimizing the back EMF filtering effect by skewing is proposed. However, in order to find the optimal skewing for the desired radial force, cogging torque and the back EMF shape the optimization process is necessary. In the next chapter, the fundamental rules for calculation the back EMF, cogging torque and radial force from numerical field computation, finite element method, is presented. In addition, a simple procedure for the calculation of the effect of the skewings from a 2$\mathrm{D}$ analysis is presented.

\subsection{Calculation of flux linkage}

Following the calculation of the winding function, the flux linkage per pole is calculated as:

$$
\lambda(\theta)=\int_{-\infty}^{\infty} W_{L A}(\tau) \cdot \phi_{g}(\theta-\tau) \cdot d \tau
$$

The concentrated windings have normally one or lower coil set per pole, $N_{c p}=1$, where the distributed windings have several coil sets per pole $N_{c p}>1$.

For the concentrated windings with full pitch winding area and, $W_{L A}=N_{w} \cdot A_{w}$, the flux linkage for a single coil is calculated as: 


$$
\lambda(\theta)= \begin{cases}-2 N_{w}\left|B_{g}\right| \cdot A_{w} \cdot \theta & 0<\theta<\frac{2 \pi}{P} \\ 2 N_{w}\left|B_{g}\right| \cdot A_{w} \cdot \theta & \frac{2 \pi}{P}<\theta<\frac{4 \pi}{P}\end{cases}
$$

where $A_{w}$ is the area of the winding function which is calculated for the central point of the slot. Here, $N_{w}$ is the number of armature winding turns per magnet pole. The number of turns, on the other hand, is connected to the current density of the machine. This is because it is the current density that determines the thermal constraint of the machine. In terms of the geometrical dimension and the current density, $J\left(A / \mathrm{mm}^{2}\right)$, the number of turns per coil, $\mathrm{N}_{\mathrm{w}}$, is defined as:

$$
N_{w}=\frac{N_{c p} \times l_{s} \times w_{s} \times J}{I_{r m s}}
$$

where $I_{r m s}$ is the root mean square of phase current. The value of the current density play intermediate role between machine power and thermal limitation.

\subsection{Calculation of back EMF of a single coil}

The induced open circuit voltage to the armature windings of the machine is the back EMF. The back EMF is calculated by means of Faraday's law as:

$$
E(\theta)=\frac{d \lambda(\theta(t))}{d t}
$$

The total back EMF in double layer winding, with all coils in series, is calculated by summation of all as:

$$
E_{T}(\theta)=\frac{d \lambda_{T}(\theta(t))}{d t}=\frac{N_{s}}{3} \times \frac{d \lambda(\theta(t))}{d t}
$$

For the single coil, concentrated winding with full pitch winding area and $W_{L A}=N_{w} \cdot A_{w}$, the back EMF is calculated as: 


$$
E(\theta)= \begin{cases}-2 N_{w}\left|B_{g}\right| \cdot A_{w} \cdot \omega_{m} \cdot \frac{P}{2 \pi} & 0<\theta<\frac{2 \pi}{P} \\ 2 N_{w}\left|B_{g}\right| \cdot A_{w} \cdot \omega_{m} \cdot \frac{P}{2 \pi} & \frac{2 \pi}{P}<\theta<\frac{4 \pi}{P}\end{cases}
$$

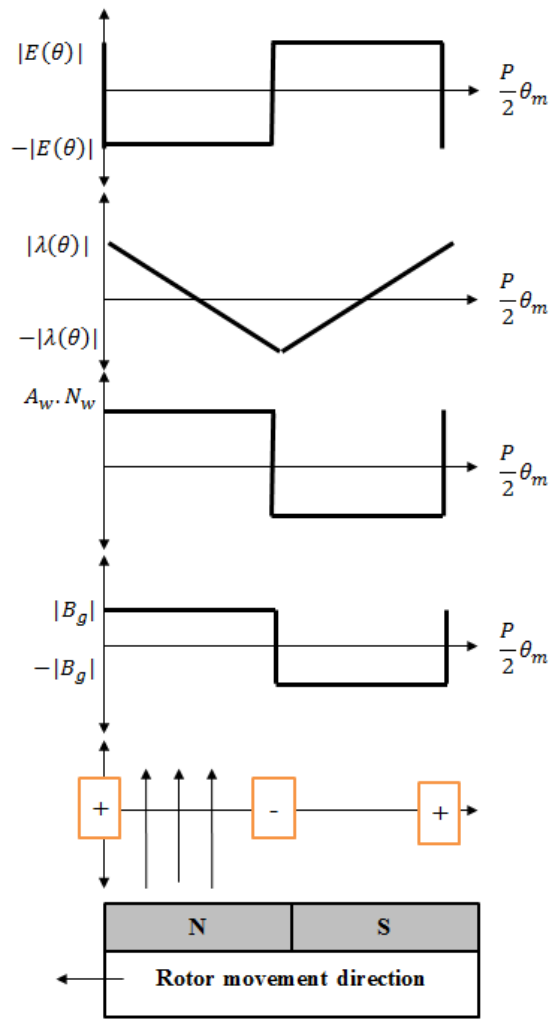

Figure 2.14 From magnet structure to back EMF creation in concentrated windings

\subsection{Calculation of power and torque for single coil}

The power of the machine for all magnetic poles for a one phase system is calculated as:

$$
\operatorname{Power}(\theta)=E_{T}(\theta) \times I_{\text {phase }}(\theta)
$$

In terms of the power and the mechanical speed, the electrical torque is calculated as:

$$
\operatorname{Torque}(\theta)=\frac{\operatorname{Power}(\theta)}{\omega_{m}}
$$


In general we can assume that:

$$
\frac{I_{\text {phase }}(\theta)}{E_{T}(\theta)}=K_{p}
$$

where $K_{p}$ is a constant number.

The above assumption means that if we are designing a machine with trapezoidal EMF, we need a step current. If we are designing a sinusoidal back EMF machine, we need AC current source. In general, in PMSM, $K_{p}<<1$, especially in high speed machines. As the $K_{p}$ is increased, the machines power density is increased. However, the heat generation and active losses of the windings also increase. By combining the (2-53) into the (2-51), therefore the power is calculated as:

$$
\operatorname{Power}(\theta)=K_{p} \times E_{T}(\theta)^{2}
$$

Equation (2-51) for the three phase machines can be restated as:

$$
\begin{aligned}
\operatorname{Power}(\theta) & =i_{a}(\theta) \times E_{T a}(\theta)+i_{b}(\theta) \times E_{T b}(\theta)+i_{c}(\theta) \times E_{T c}(\theta) \\
& =K_{p} \times\left[E_{T a}(\theta)^{2}+E_{T b}(\theta)^{2}+E_{T c}(\theta)^{2}\right] \\
& =K_{p} \cdot \max \left(E_{T}{ }^{2}\right) \cdot\left[\sin (\theta)^{2}+\sin (\theta+120)^{2}+\sin (\theta+240)^{2}\right] \\
& =\frac{3 K_{p} \max \left(E_{T}{ }^{2}\right)}{2}
\end{aligned}
$$

According to equation (2-48) and (2-55), in a three phase machine, the output power is related to the number of the windings turn per magnet poles, maximum current density and air gap flux density. On the other hand according to the (2-48) and (2-54), the power of the machine is connected to the maximum back EMF. The maximum back EMF is linked to the maximum speed and the flux linkage as well as the number of coils. The flux linkage is connected to the winding factor and to the reluctance network through equations (2-1) to (2-29). Consequently, in the classic design, the nominal power, the nominal torque and the maximum speed are assumed 
initially. Then equations (2-55) to (2-1) are reversely followed to obtain the dimensions of the machine in an iterative process.

\subsection{Summary}

The slot pitch, pole pitch, permanent magnet demagnetization and the dimension of the machine and the number of the turns/coil are the factors that affect the flux linkage. As the result, the back EMF of the machine is also affected. The back EMF directly affects the power of the machine from equation (2-55). Finding the best back EMF shape and amplitude for a nominal speed and torque concerning a multiobjective consideration of cogging torque, radial force and thermal limitation require an optimization process. Although, the optimization process can be launched from the introduced classic design procedure for the desired objectives like minimum cogging torque, minimum radial force and proper back EMF waveforms, the difficulties in the accurate calculation of the back EMF harmonics, cogging torque amplitude, radial force amplitude encourage us to expand the design problem into a numerical domain. In addition, the mutual integration of the drive and the machine being developed is one of the subjects that cannot be easily calculated from analytical expressions. Therefore, the classic design seems to be just a reasonable state for initialization of the design. In other words, the classic design enables us to quickly obtain a normal estimation of the final design. In the initial design stage, the general geometry of the machine, the initial dimension and the material for the nominal operating point are calculated based on the knowledge presented in this chapter. The final design comes from the advanced optimization process which covers higher dimensional objectives. 


\section{Chapter 3 Modeling of Permanent Magnet Synchronous Machine (PMSM)}

\subsection{Overview}

The co-design procedure of a PMSM includes employing a functional model of the machine that covers the thermal, electrical and magnetic aspects of the machine. In the next chapters it will be shown that how the physics-based model is employed in the heart of the design optimization system, observers, diagnosis and prognostic system.

The physics-based model, by definition, is an FE database model that can predict the machine operational as well as the internal conditions at different working points. The physics-based model could monitor the thermal, electrical and permanent magnet operating point in the real-time environment.

In this chapter, three low frequency electrical models of a PMSM are presented. These are to be utilized in solving the integrated motor drive system. The first method is the coupled field circuit method. The second method is called the physics-based method. And the third method is the well-known average $d-q$ model. The average $d-q$ model is utilized in design of speed control loop. And the other two models are employed for the verification and realization of the developed theories. The physicsbased method has superior characteristics to the coupled-field circuit method in the operational environment. The level of accuracy and the details in this model depends on the dimensions of the employed look-up Tables. That is why the physics based model is utilized for the development of physics-based observers, physics-based design optimization system and physics-based fault diagnosis and prognostic systems. The coupled field-circuit model is employed for verification of the physics-based model. The methods are implemented in the Simulink enviornment. In the coupled 
field circuit model, a functional block representing the FE model of the machine is directly coupled with external power electronics and the control system using the software "Magnet 2D"-Simulink link. The input to the block is voltage and load torque while the outputs are the phase currents and other mechanical quantities such as speed and position. The same time step was used to solve the field, circuit, power electronics and control equations together. The circuit equations in the FE domain consist of standard conductors, nonlinear core material and demagnetization models. The benefits obtained by this method are the ease of model construction and simulation, flexible options for post processing and the possibility of extending the finite element analysis to experts in power electronics or control systems without a comprehensive knowledge of FEM. This method is mainly used for the verification of the physics-based model.

In the physics-based model, the electrical machine is characterized by either differential equations or circuit elements. The parameters required by the model such as back EMF, dynamic inductance, cogging torque and cogging flux linkage and transfer demagnetization inductances are solved by direct-coupled field circuit analysis at each time step. A ladder thermal model was developed to predict the permanent magnet and winding temperature. In addition, a transfer demagnetization inductance in developed to measure the effect of stator winding loading on the permanent magnet remnant flux. Having the winding temperature the winding resistance is updated during simulations. Having the magnet temperature and the effect of armature reverse field on magnet, the magnets operating point is predicted. To calculate the required parameters for the physics-based model, coupled field and circuit equations for electromagnetic conductors are solved using nonlinear transient solutions. 


\subsection{Slow time-varying fields}

The slow varying fields are understood to be the field that does not lead to current redistribution [112-114]. On the other hand, we can say that there are no eddy current effects as the dimensions of the current carrying conductors that are smaller than the penetration depth of the field. The currents at those frequencies are distributed as in the DC case, uniformly over the whole surface of the conductor. Eddy current effects are included in the field equation with fast varying time dependence. The slow varying field belongs to as quasi-stationary group. Most of the electrical phenomena in energy systems can be located in quasi-static group. Slowly varying and periodic fields up to $10 \mathrm{kHz}$ are considered as quasi stationary. Electrical machines are in this group. In the quasi static fields the current displacement inside the conductive parts is negligible and the magnetic field $H$ outside the coil is exclusively excited by the free current density $J$. For the quasi-static fields, the Ampere law is valid.

$$
\nabla \times H=J+\left(\frac{d D}{d t} \approx 0\right)
$$

The determination of the quasi static field depends on our choice that whenever the current displacement $d D / d t$ can be ignored. It depends on the wavelength of the problem deliberated in frequency domain. If the wavelength is large enough compared to the physical dimension of the problem the field problem is called in the quasi-static problems. The quasi-static problems can be static, slowly varying transient and timeharmonic eddy current. All of these three categories can be used in low frequency modeling of electrical machines.

\subsection{Magneto dynamic fields (low frequency quasi-stationary fields)}

The basic laws of magneto-dynamic fields are Ampere's law, without consideration of time variation of electric displacement flux density $D$ i.e. $d D / d t \approx 0$ 
, Faraday's law, Magnetic flux continuity and the constitutive relations [112-116], see the Appendix II. In term of magnetic vector potential the magnetic flux density is:

$$
B=\nabla \times A
$$

By substituting equation (3-1) in Faraday's law we can obtain:

$$
\nabla \times E=-\frac{d B}{d t}=-\frac{d(\nabla \times A)}{d t}
$$

Now employing the Ohm law to calculate the eddy current $J_{e}$ yields:

$$
J_{e}=\sigma \cdot E=-\sigma \cdot \frac{\partial A}{\partial t}
$$

The Ampere's law can be rewritten by using equations (3-2) and (3-3), yielding the $A$-formulation for the quasi-stationary magnetic field in time domain:

$$
\nabla \times\left(\frac{1}{\mu} . \nabla \times A\right)+\sigma \cdot \frac{\partial A}{\partial t}=J_{e}
$$

The equation (3-6) is used for simplification as:

$$
\nabla \times(\nabla \times A)=\nabla(\nabla \bullet A)-\nabla^{2} A
$$

Using (3-6) in (3-5) and assuming coulomb gauge condition leads to:

$$
\nabla^{2} A-\mu . \sigma \cdot \frac{\partial A}{\partial t}=-\mu . J_{e}
$$

To include the magnetic material in equation (3-5) or (3-7), the demagnetization characteristic can be defined by:

$$
B=\mu_{0}((1+\varsigma) H+M)
$$

where the $\zeta$ is the magnet susceptibility, $M$ is the magnetization vector and $H$ is the field strength at the operating point. From (3-8) the remnant flux density is defined by:

$$
B_{r}=\mu_{0} \cdot M
$$


The incremental permeability is the slope of the demagnetization characteristic. It is:

$$
\frac{\partial B}{\partial H}=\mu_{0}(1+\varsigma)
$$

$\zeta$ is a very small positive number so that the apparent permeability of magnet is only slightly larger than the free space. The reluctivity is defined as:

$$
v=\frac{1}{\mu_{0}(1+\varsigma)}
$$

By applying equation (3-11) into the demagnetization characteristic, equation (38), we may have:

$$
H=v \cdot\left(B-\mu_{0} \cdot M\right)
$$

The Ampere law for a magneto static problem is:

$$
\nabla \times H=J_{e}
$$

Using (3-12) in (3-13) yields to:

$$
\nabla \times(v \cdot B)-\nabla \times\left(v \cdot \mu_{0} \cdot M\right)=J_{e}
$$

The term $\nabla \times\left(v \cdot \mu_{0} \cdot M\right)$ represents an equivalent source term, $J^{*}$ and can be identified as an equivalent magnetic current. Including this current source in equation (3-5) and assuming $\nu . \mu_{0}=\mu$ yields to the following:

$$
\sigma \cdot \frac{\partial A}{\partial t}+\nabla \times\left(\frac{1}{\mu} \cdot[\nabla \times(A-M)]\right)=J_{e}
$$

If there is motion of any material within the system, we can use the empirical expression for transformation of electric field between observers moving with respect to each other. Here the slave frame is moving with respect to the master frame by velocity of $\vartheta[112]$ : 


$$
E^{\prime}=E+\vartheta \times B
$$

Rewriting of equations (3-2) to (3-15) for $E^{\prime}$, the new magnetic field formulation is a achieved as:

$$
\sigma \cdot \frac{\partial A}{\partial t}+\nabla \times\left(\frac{1}{\mu} \cdot[\nabla \times(A-M)]\right)-\sigma \cdot \vartheta \cdot(\nabla \times A)=J_{e}
$$

Equation (3-17) is the complete form of the magnetic vector potential which contain magnetic material and the motion. Evidently, the areas that do not include the motion or the magnetic sources, their respective current density are removed.

\subsubsection{Calculation of stored magnetic energy}

From the Poynting's theorem the magnetic power volume density $p_{m}$ is assumed as:

$$
p_{m}=H \cdot \frac{\partial B}{\partial t}
$$

The corresponding magnetic energy volume density $w_{m}$ is calculated as:

$$
w_{m}=\int_{t=0}^{t_{1}} p_{m} d t=\int_{t=0}^{t_{1}} H \cdot \frac{\partial B}{\partial t} d t=\int_{B=0}^{B_{1}} H \cdot d B
$$

The corresponding stored magnetic energy is the integral over the entire volume of the problem of the magnetic energy density as:

$$
W_{m}=\int_{V} w_{m} \cdot d V
$$

According to (3-19), the energy is related to the area under the $H$ versus $B$ curve, i.e., at a point where the magnetic flux density is $B_{1}$, the magnetic energy volume density is:

$$
w_{m}=\int_{B=0}^{B_{1}} H(B) \cdot d B
$$


In this expression, $H(B)$ is the magnetic field intensity for a given magnetic flux density $B$. For isotropic soft magnetic materials, $H$ and $B$ are linear i.e. they always in the same direction and the energy density is positive. On the other hand, the energy density may be negative if permanent magnets are present.

\subsubsection{Force calculation}

A set of touching or embedded components completely surrounded by the material air, or up against an outer boundary is called body. The net force vector $F$ calculated for a solid body inside the machine results from the integration of the electromagnetic force density vector field over that body. The influence of the force density vector field on the body is the same as $F$ acting on the center of mass of the solid body, plus a torque vector with respect to the same point. There are three different methods that can be used to obtain the electromagnetic forces on bodies or

components. These are Lorentz, Maxwell stress tensor and Energy methods [117$125]$.

\subsubsection{Lorentz method for calculation of force}

This method is normally used to analyze the force when the materials are not permeable (i.e. when $\mu_{r}=1$ ). The Lorentz force density, $J \times B$, is considered only for current carrying components. On current carrying components, the force can be calculated using the Lorentz force density:

$$
f=J \times B
$$

From which we find the total magnetic force acting on a body by integration of (322) over its volume $V$ :

$$
F=\int_{V} f . d v=\int_{V} J \times B . d v
$$




\subsubsection{Maxwell stress for calculation of the force}

This method can be used to calculate the force almost for all bodies, regardless of the material. When the force on some components building up the body can be computed with the Lorentz method, the composite force on the body's other components can be gained from the vector subtraction of the Maxwell stress body force and the Lorentz forces. The Maxwell stress method is used to evaluate electromagnetic forces on bodies, based on an algorithm requiring that the body be completely surrounded by air, although it can touch some model boundaries. The Maxwell Stress force computation on a body is performed over the layers of air elements that are directly in contact with the body. This means that the computation ignores the boundaries of the body that lie on the outer boundary of the model, since there are no air elements in contact with those boundaries. However, it also means that the computation takes into account the surfaces of any air holes within the body, these surfaces being viewed as so many additional exterior boundaries [117].

On current carrying components, the force can be computed using the Lorentz force density from equation (3-22). From which we obtain the total magnetic force acting on a body by integrating over its volume $V$ from equation (3-23). From Ampere's law we have:

$$
J=-\nabla \times B / \mu
$$

When displacement currents are neglected for slow varying field, the usage of equation (3-24) in the (3-23) leads to:

$$
F=\frac{-1}{\mu} \int_{V} \nabla \times B \times B . d v
$$

In equation (3-25) it is shown that: 
$-\nabla \times B \times B=\nabla . T$

where $T$ is the second Maxwell stress tensor and can be defined by:

$T=B \cdot B-\frac{B^{2} \cdot I}{2}$

with $I$ the unit tensor. Indeed, we have:

$\nabla \cdot T=\nabla \cdot\left(B \cdot B-\frac{B^{2} \cdot I}{2}\right)=B \cdot \nabla \mathrm{B}+\nabla \cdot B \cdot B-\frac{1}{2} \nabla\left(B^{2}\right)$

Since $\nabla \bullet(A B)=A \bullet \nabla B+\nabla \bullet A B$ and $\nabla \bullet(a I)=\nabla a$ then:

$\nabla . \bullet T=B \bullet \nabla \mathrm{B}-B \bullet \nabla B$

Because $\nabla \cdot \bullet B=0$ and $\nabla\left(B^{2}\right)=2 B \nabla B$ then:

$\nabla \bullet T=-B \times \nabla \times \mathrm{B}$

Using $A \times(\nabla \times \mathrm{B})=\nabla B \bullet A-A \bullet \nabla \mathrm{B}$ and using the divergence theorem leads to:

$$
F=\frac{1}{\mu} \int_{V} \nabla \bullet T . d v=\frac{1}{\mu} \int_{S} T . d s
$$

where $S$ is the bounding surface of the body, this surface being entirely located in regions with relative permeability of 1 .

When the body encloses one or more air holes, the influence of the holes on the integrated force $F$ is theoretically zero. Indeed, the portion of the bounding surface $S$ in a body's hole can be arbitrarily selected as long as it is entirely confined in air and could thus be selected as becoming smaller and smaller in the middle of the hole until it is reduced to a point with zero area, which does not add any involvement to the resulting integrated force $F$. In using the Maxwell stress method to compute the force on a body, the surface integration in (3-31) may be performed on different surfaces surrounding the body and the resulting forces should theoretically be exactly the same 
as long as the relative permeability is unity in the surrounding region. In practice, however, the forces will be different because of local field errors, which can at times be very large. As an attempt to reduce the effect of these errors, it is beneficial to perform over the first layer of surrounding air elements a volume integral that amounts to averaging the forces calculated using many adjacent surfaces located in this first layer.

\subsubsection{Energy method}

The force can be easily achieved from the energy and a small displacement of the object of interest. The energy technique is founded on the space variation of the total energy when the object of interest is moved. If the stored energy is evaluated for the device in two positions separated by a small displacement provided the flux remains constant, then the difference in energy divided by the distance will give a value for the force. This approach to force calculation is widely employed in mechanics, where it is known as the method of virtual work. It relies on the assumption that the force does not change significantly during the motion and is thus valid for small displacements only. In the completely general case where displacements may take place in various directions, the force is a vector quantity, given by:

$$
F=\nabla W
$$

where $W$ is the total stored magnetic energy of the system, viewed as a function of the vector displacement $r$.

The validity of this method relies on the assumption that the force does not change significantly during the movement. The chosen locations should thus be close enough from each other but yet exhibit large enough energy differences in order to avoid a "catastrophic cancellation effect". A 5\% difference in energy is usually adequate. Since the FEM is based on the minimization of energy, the energy method is a good 
way to compute the force because it uses the total energy, which is usually correctly predicted. Moreover, several devices (e.g. motors) are usually solved at different positions and this lends itself well to the energy method. In order to get satisfactory force values using the energy method, the object on which the force is calculated should be translated at several neighboring locations around the position of interest and for each of them the energy should be taken. If a smooth curve is fitted to these several values, a more accurate gradient can be computed than by using a simple finite difference. In non-linear_problems, the current should be considered as a fixed quantity and therefore, the co-energy_is used to obtain the force by the energy method.

\subsubsection{Torque calculation}

The torque on a body is computed about the origin using:

$$
T_{0}=\int_{V} r_{0} \times f d v
$$

where $r_{0}$ is the vector going from the origin to an element of volume in the body, $V$ is the volume of the body and $f$ the force density[119], [121], [126].

\subsubsection{Cogging torque[101]}

Each time a rotor pole comes into line with a slot or a tooth, the torque will be zero by symmetry. As a result, the cogging torque period is the angle between two slot alignments. In one revolution of the rotor, each pole will bring into line with each slot at some point, hence there will be $P \times N_{s}=N_{a}$ number of alignments, where $P$ and $N_{s}$ are the number of poles and number of slots, respectively. Though, several

poles may align concurrently, in fact, $G C D\left(N_{s}, N_{a}\right)$ will align concurrently each time. Thus, the cogging torque period $T_{C}$ is given by: 


$$
T_{C}=\frac{2 \pi}{N_{a}}
$$

34)

$$
N_{a}=\frac{P \times N_{s}}{G C D\left(P, N_{s}\right)}
$$

The cogging torque is normally a small amount. For instance, a representative running torque is of the order of $10 \mathrm{Nm}$, whereas the corresponding cogging torque will be a ripple (always present in the running torque-angle curve with amplitude of the order of $0.1 \mathrm{Nm}$. It is therefore challenging to simulate the cogging torque precisely since a high accuracy level is required. Two methods are generally used in order to simulate the cogging torque:

- Execute a series of static simulations with the rotor angle parameterized in order to obtain the torque as a function of the rotor angle.

- Run a single velocity driven, transient with motion simulation and obtain the torque as a function of time. This method of solving the static parameterized transient problem with motion solver offer several advantages.

In both cases, the stator coils are set as being open-circuit. Simulated cogging torque is slightly smaller than the measured values. The reason is that the cogging torque is simulated with a model demonstrating a perfect motor, for instance with magnets perfectly positioned and having perfectly uniform and well-directed magnetizations. This theoretical cogging torque is very small when the motor is designed to minimize it, so it is likely that the measured cogging torque might essentially be due to slight imperfections in the magnets. In this case, the measured cogging torque will not have a simple period corresponding to the theoretical period and this can be confirmed by looking at the measured torque data. It is not usually 
possible to simulate the small imperfections in the actual motor because this would require a detailed knowledge of the imperfections, which is usually not available.

In order to calculate the effect of the skewing, 3-D FE analysis is required. Here a simplified solution is proposed to calculate the cogging torque of a skewed stator from the 2-D field analysis. If we have a machine with linear skewed stator slot, the teeth opening rotate according to skew angle as well. Therefore, the cogging torque rotates with skew angle in different axial length. Here the normalized cogging torque without the skewing is defined as:

$$
T_{C N}(\theta)=\frac{T_{C o g}(\theta)}{L_{s t}}
$$

If the teeth opening skewed linearly along the axial length, $l$, of the machine, then the skew angle can be defined as:

$$
\theta_{\text {skew }}=\frac{\alpha . l}{L_{s t}}
$$

where the $L_{s t}$ is the stack length and the $\alpha$ is the maximum skew angle. The resultant cogging torque is calculated by summation of all the normalized cogging torque values along the axial direction as:

$$
T_{\text {Cog }}=\int_{0}^{L_{s t}} T_{C N}\left(\theta-\frac{\alpha \cdot d l}{L_{s t}}\right)
$$

Equation (3-38) is valid as linear value similar a skew factor, equation (3-37), is created in the magnet poles.

\subsubsection{Calculation of flux linkage}

For a single filamentary current $\operatorname{loop} C$, the flux linkage $\phi$ is given by[127], [128]: 


$$
\phi=\int_{S} B \cdot d s=\int_{S} \nabla \times A \cdot d s=\oint_{C} A . d l
$$

where $A$ is the vector potential. For a stranded coil, the summation of the flux leads to:

$$
\phi=\sum_{\text {turns }_{C}} \oint_{A} A d l
$$

If there are $n\left(\right.$ turns $\left./ m^{2}\right)$ in the cross-section $S_{C}$ of a coil, the equation (3-40) converts to:

$$
\phi=\int_{S_{C} C} A \cdot d l \cdot n \cdot d S
$$

If each turn carries current $I$, then the current density is $J=n . I$. Also, $d l . d S=d v$, and therefore $J . d v=n . I . d l . d S$. Then, the flux linkage becomes:

$$
\phi=\frac{1}{I} \int_{V_{C}} A . J . d v
$$

where $V_{C}$ is the volume of the coil.

For stranded coil, the flux linkage corresponding to the total flux passing all the turns is calculated as:

$$
\lambda=N \cdot \phi
$$

\subsubsection{Calculation of back EMF}

The derivation of the flux linkage resulted just from magnets is called back EMF. The EMF is calculated as[129]:

$$
\operatorname{EMF}(\theta(t))=\frac{d \lambda\left(\theta_{r}(t)\right)}{d t}
$$

In the case of skewing described in equation (3-37), the EMF can be calculated from 2-D FE analysis by additional post processing as: 


$$
\operatorname{EMF}\left(\theta_{r}(t)\right)=\int_{0}^{L s t} \frac{E M F}{L_{s t}}\left(\theta_{r}(t)-\frac{\alpha \cdot d l}{L_{s t}}\right)
$$

where $\theta_{r}$ is the electrical angular position.

\subsubsection{Inductance calculation}

The low frequency inductances of electrical machines can be calculated from a non-linear transient FE analysis with motion. It also can be calculated either by static FE analysis, or analytical approaches[93], [130-132]. The FE approaches are the most reliable approaches since they could include the non-linearity in the solution. The inductance can be calculated from a single static FE analysis of the machine. However, the main misunderstanding in calculation of inductance from a single static FE analysis is the under-estimation of the saturation level, the role of the machine saliency and rotor position in inductance profile. In general, there are two solutions to this problem. The first solution is to perform a non-linear FE solution for a specific instantaneous current level and rotor angle. Following that, the permeability of each finite element is frozen and the linear solutions are done to calculate the inductance in that corresponding instantaneous current levels and rotor position. The calculated inductances represent the saturated level for that specific operating point. It means that an equivalent machine that has a 'linear' characteristic around the operating point has replaced the saturated machine. This equivalent machine has appropriate values of inductance, but the frozen permeability of finite elements must be updated if the machine moves significantly from the operating point that was used for the inductance calculation. Infinite solutions are required to calculate the continuous inductances for all current levels and rotor positions, although doing this for a discrete set of current level and rotor position and then linear or cubic interpolations will be a reasonable alternative approach. The second solution is basically based upon the assumption that 
the inductance does not change due to a small increment in the current level. Based upon this assumption of incremental flux linkage method or energy perturbation methods are well defined to calculate the inductance at a specific saturation level, instantaneous current level and rotor angle. To perform this, at first a nonlinear FE solution corresponding to a snapshot of current level and rotor angle is computed. A signature from machine such as flux, flux linkage or energy is kept. Another nonlinear FE solution is performed while a small current increment is added to the current level used in the first step. Again the same signatures from the machine are extracted and the values of inductances are calculated based on the difference of the new and old flux, energy or flux linkage definitions.

\subsubsection{Flux linkage method for inductance calculation}

From Faraday's law for the case of a single-turn coil with no internal resistance, we have:

$$
V=\frac{d \phi}{d t}
$$

If the coil is replaced by an N-turn closely wound coil, each loop will be linked by the same flux and will thus be the location of an induced EMF given by (3-46). Since all these EMF's are in the same direction, they will add up in series so that the total EMF induced in the coil will be:

$$
\begin{aligned}
& V=\frac{d \phi}{d t} \\
& V=N \frac{d \phi}{d t}=\frac{d \lambda}{d t}
\end{aligned}
$$

where according to (3-43) $\lambda=N \phi$ is the total flux linking the coil.

Now, supposing that the only time-varying quantity is the coil's current, therefore: 


$$
\lambda=\lambda\left[I(t), B_{0}(r)\right]
$$

where $B_{0}(r)$ is the non-uniform, stationary magnetic field remaining when $I(t)=0$ and caused by permanent magnets or coils with fixed current. Substituting (3-49) in (3-48) and using the chain rule leads to:

$$
V=\frac{d \lambda}{d I} \cdot \frac{d I}{d t}
$$

By defining the incremental inductance $I$ as:

$$
l=l\left[I(t), B_{0}(r)\right] \equiv \frac{d \lambda}{d I}
$$

The equation (3-50) can be re-written as:

$$
V=l \cdot \frac{d I}{d t}
$$

52)

The incremental inductance provides the change in flux corresponding to a small change in current (i.e. it is the local slope of the $\lambda(I)$ curve) about a particular operating point $\left(I, B_{0}\right)$ as well as the proportionality factor between the voltage and the proportion of change of the current shown in (3-50). If, in addition to the "background field coils "n" other coils are present, equation (3-48) is replaced by:

$$
V_{i}(t)=\frac{d \lambda_{i}(t)}{d t}
$$

where the flux linking coil " $\mathrm{i}$ " is:

$$
\lambda_{i}=\lambda_{i}\left[I_{1}(t), \ldots, I_{n}(t), B_{0}(r)\right]
$$

In (3-54), $I_{1}(t), \ldots, I_{n}(t)$ are the currents in each of the " $n$ "coils. Substituting (3-54) in (3-53) and using the chain rule for several variables leads to: 


$$
V_{i}(t)=\sum_{j=1}^{n} l_{i j} \frac{d I_{j}(t)}{d t}
$$

where the incremental inductance is this time set by:

$$
l_{i j}=l_{i j}\left[I_{1}(t), \ldots, I_{n}(t), B_{0}(r)\right] \equiv \frac{d \lambda_{i}}{d I_{j}}
$$

where $l_{i j}$ corresponds to the rate of change of $\lambda_{i}$ (flux linking coil " $i$ ") with respect to a small change in $I_{j}$ (current in coil “j”) about a specific operating point $I_{1}(t), \ldots, I_{n}(t), B_{0}(r)$

For a single coil consider the linear situation where $l=L$ is a constant. In this case, (3-54) can in general be written as:

$$
\lambda=L . I+\lambda_{0}
$$

where $L$ is the absolute inductance or simply the inductance and $\lambda_{0}$ resembles to a constant flux linkage through the coil, caused for example by the presence of a permanent magnet. If $\lambda_{0}=0$, one simply has:

$$
\lambda=L . I\left(\lambda_{0}=0\right)
$$

If $\lambda_{0} \neq 0$, one can implement a couple of static FE analysis solutions or a transient solution at a few time instants in order to obtain fluxes $\lambda_{1}$ and $\lambda_{2}$ corresponding to two different excitation currents $I_{1}$ and $I_{2}$. Inserting these values in (3-57) gives:

$$
\begin{aligned}
& \lambda_{1}=L \cdot I_{1}+\lambda_{0} \\
& \lambda_{2}=L \cdot I_{2}+\lambda_{0}
\end{aligned}
$$

and subtracting equation (3-60) from (3-59) leads to:

$$
L=\frac{\lambda_{2}-\lambda_{1}}{I_{2}-I_{1}}=\frac{\Delta \lambda}{\Delta I} \quad\left(\lambda_{0} \neq 0\right)
$$


Note that (3-61) corresponds to a local infinitesimal slope, those changes from one operating point to the other in the general nonlinear case.

\subsubsection{Energy method}

If there is a single energized coil in a model, the total stored magnetic energy $W$ can be employed to obtain its inductance (or the equivalent inductance of several coils) using the total current entering the machine. In order to show this, we start from the general expression relating the total magnetic energy $W$ and the total magnetic power $P$ :

$$
W=\int P \cdot d t=\int V \cdot I \cdot d t=\int l \cdot \frac{d I}{d t} \cdot I \cdot d t=\frac{1}{2} \int l \cdot \frac{d\left(I^{2}\right)}{d t} \cdot d t=\frac{1}{2} \int l \cdot d\left(I^{2}\right)
$$

From (3-62) the expression for the incremental inductance is defined by:

$$
l=2 \frac{d W}{d\left(I^{2}\right)}
$$

In the general, for nonlinear cases, similar techniques to those proposed for the flux linkage approach can be used with (3-63). For the linear case, where $l=L$ a constant is, we have from (3-62):

$$
W=\frac{1}{2} \cdot L \cdot I^{2}+W_{0}
$$

where $L$ is the absolute inductance or basically the inductance and $W_{0}$ resembles a constant energy term, caused for example by the presence of a permanent magnet. If $W_{0}=0$, one simply has:

$$
L=\frac{2 . W}{I^{2}}\left(W_{0}=0\right)
$$

If $W_{0} \neq 0$, one can perform a couple of static $\mathrm{FE}$ solutions or a transient $\mathrm{FE}$ solution at a few time instants in order to obtain the energies $W_{1}$ and $W_{2}$ 
corresponding to two different excitation currents $I_{1}$ and $I_{2}$. Inserting these values in (3-64) gives:

$$
\begin{aligned}
& W_{1}=\frac{1}{2} \cdot L \cdot I_{1}^{2}+W_{0} \\
& W_{2}=\frac{1}{2} \cdot L \cdot I_{2}^{2}+W_{0}
\end{aligned}
$$

By using (3-67) and (3-66), we finally have:

$$
L=2 \frac{W_{2}-W_{1}}{I_{2}^{2}-I_{1}^{2}}=2 \frac{\Delta W}{\Delta I^{2}} \quad\left(W_{0} \neq 0\right)
$$

\subsubsection{Impedance method}

The impedance is a third way to compute the inductance. Considering first the linear case, utilizing (3-55) for a single coil leads to:

$$
V=L \cdot \frac{d I}{d t}
$$

In a Time-harmonic context, the excitation voltage is sinusoidal periodic function, $V=V_{0} \cdot \cos (\omega t)$ and the resulting current has the form of $I=I_{0} \cdot \cos (\omega \cdot t)$. One can use phasors so that (3-69) will correspond to the real part of the complex equation:

$$
V=j \omega L . I
$$

where the complex voltage $V$ and current $I$ are given by

$$
\begin{aligned}
& V=V_{0} \cdot e^{j \omega t} \\
& I=I_{0} \cdot e^{j(\omega t+\varphi)}
\end{aligned}
$$

From which we have:

$$
L=\frac{1}{\omega} \cdot \operatorname{Im} g\left(\frac{V}{I}\right)=\operatorname{Im} g\left(\frac{V_{r m s}}{\omega \cdot I_{r m s}}\right)
$$

Note again that $\mathrm{V}$ and I are complex quantities. When the excitation is sinusoidal, 
equation (3-71) can be used at steady-state (i.e. once the non-repeating transients have stopped) even in a nonlinear transient context by taking the response signal's fundamental in order to obtain what would correspond to the inductance $L$ in a linear context. This is done by expressing the complex voltage and current in the time domain upon substitution of (3-71) and (3-72) into (3-73) leads to:

$$
L=\left(\frac{-V_{0} \cdot \sin (\varphi)}{\omega \cdot I_{0}}\right)
$$

In (3-74), $I_{0}$ is the amplitude of the current's fundamental and $\varphi$ is the phase shift between the current and voltage fundamentals. Note that for an inductance, $\varphi<0$ since the current lags the voltage.

\subsubsection{Open-circuited inductance calculation method}

If it is desired to find the inductance of an open-circuited coil, i.e. when $I=0$, none of the above methods can be used. In this case, one could set a very small perturbation current so that it would not perturb the fields. In the linear case, for which the inductance is independent of the current, the current can be set to any value.

\subsubsection{Voltage method}

The voltage method is based upon synchronous solutions of two separate successive nonlinear transient FE solutions with motion coupled with external circuit. This technique is a circuit-based inductance calculation one that uses the induced voltages in the windings due to the rotating rotor and a set of DC currents to evaluate the inductance at different rotor positions and saturation levels. This procedure can be summarized as:

Step1- Solve equations (3-17), (3-75) and (3-76) using a nonlinear transient FE analysis with motion coupled to circuit. This is done while the conductivity of the 
winding area is set to infinity. This assumption is based on the fact that the inductance calculation system does not include active losses and Ohmic voltage drops. This solution is done for a snapshot of current level for each discrete degree of rotations for a full electrical cycle. The dominated circuit equation can be expressed as:

$$
V_{1}=\frac{d \lambda}{d t}=\frac{d\left(\lambda_{s}+\lambda_{r}\right)}{d t}=\frac{d\left(l_{1} \cdot I+\lambda_{r}\right)}{d t}=I \cdot \frac{d\left(l_{1}\right)}{d t}+\frac{d\left(\lambda_{r}\right)}{d t}
$$

Step2- Repeat step one exactly for the same situation while the current in one of the phases is increased by a small increment $\varepsilon$. The dominated circuit equation similarly can be expresses as:

$$
V_{2}=\frac{d \lambda}{d t}=\frac{d\left(\lambda_{s}+\lambda_{r}\right)}{d t}=\frac{d\left(l_{2} \cdot(I+\varepsilon)+\lambda_{r}\right)}{d t}=(I+\varepsilon) \cdot \frac{d\left(l_{2}\right)}{d t}+\frac{d\left(\lambda_{r}\right)}{d t}
$$

where $I$ is the current and represent the saturation level, $\lambda_{r}$ are the pure magnet's

flux linkages, $\lambda_{s}$ are flux linkages due to stator winding and $V$ are the sub-winding and windings voltages.

If it is assumed that, the incremental current of one of the phases, $\mathcal{E}$, does not change the inductance of the machine for all of rotor positions i.e. $l_{1}=l_{2}=l$, then from equations (3-75) and (3-76) the corresponding inductance of that phase are calculated as:

$$
l=\int_{0}^{T k}(\varepsilon)^{-1} \cdot\left(V_{2}(\tau)-V_{1}(\tau)\right) \cdot d \tau
$$

\subsubsection{Coupling of slow varying field and circuit equations[93]}

In the general case, the magnetic and electric equations are solved simultaneously. The electromagnetic field equations are solved using a formulation with magnetic vector potential. The coupling is achieved by the conductor current expressed in terms of current density and the flux linkage expressed from the vector potential. Two 
approaches can be considered for choosing the unknown of the coupled system. In the first approach, the coupled equation system is only expressed in terms of the vector potential and scalar potential. In this approach, the resultant equations are symmetric positive definite but the electric circuit non-linearity cannot be reflected simultaneously with that of the magnetic circuit. In the second approach, the magnetic variables and the currents are kept as unknowns. In this approach, the resultant coupled equations are not positive definite, but the non-linearity of the magnetic materials and electric circuit can be considered simultaneously.

\subsubsection{Stranded conductors[133]}

The filamentary conductor sometimes referred to as stranded conductor. It consists of several turns of thin wire carrying the same current. In order to simplify the analysis, the eddy currents in filamentary conductors are not taken into account, but a constant current density is assumed. They are assumed too thin to allow eddy currents to appear. If we have a region k with $N . S_{k}$ filaments carrying a current $I_{k}$ and of area $S_{k}$, the current density will be considered as constant:

$$
J_{k}=\frac{N \cdot S_{k} \cdot I_{k}}{S_{k}}
$$

\subsubsection{Boundary conditions[134]}

The finite element (FE) tries to find the unknown function $\phi$ from a partial differential equation (PDE). In addition, $\phi$ should satisfy the equation $L . \phi=g$ within the prescribed solution region $R$. The choice of the boundary conditions not only influences the final solution, but can further reduce the analysis domain. The boundary condition can be imposed by three main groups:

Dirichlet boundary condition: it is characterized by assigning a determined value 
of the potential on a given boundary curve. In this way, this curve is characterized by a constant value of the potential. The equi-potential lines are tangential to such a boundary, in other words, no lines crosses this boundary. The Dirichlet condition is expressed as:

$$
\phi(r)=f(r)
$$

Neumann condition: This condition is assigned by fixing the normal derivative of potential on a given boundary curve. In other words, the line crosses the boundary in a known way.

The Neumann condition is expressed as:

$$
\frac{\partial \phi(r)}{\partial n}=g(r)
$$

The suitable choice of boundary conditions and the choice of periodic conditions can help reduce the analysis domain. For example in multi-pole symmetrical rotating machines the field analysis can be reduced to even number of the pole by using the periodic condition. For a $P / 2$ pole pair electrical machine in polar coordinate, the periodic boundary conditions are:

$$
A(r, \theta)=A\left(r, \theta+2 . k \cdot \frac{2 \pi}{P}\right) \quad(k=1,2,3, \ldots)
$$

The analysis can further be reduced to an odd number of poles by employing antiperiodic boundary conditions.

$$
A(r, \theta)=-A\left(r, \theta+2 .(k-1) \cdot \frac{2 \pi}{P}\right) \quad(k=1,2,3, \ldots)
$$

The periodic and anti-periodic boundary conditions are the family of Neumann boundary condition.

3.3.11 Finite element solution for slow time-varying fields [93], [133-136] 
Equation (3-17) represents the fundamental equations of the vector potential formulation for electromagnetic field. This equation is then solved using FEA which involve the following four steps for any problem:

- Discretizing the solution region into a finite number of sub-regions or elements.

- Deriving governing equations for a typical element.

- Assembling all elements in the solution region.

- Solving the system of equations.

The two-dimensional geometry is meshed by finite elements, consisting of first or second order triangular and quadrilateral elements. If possible, the cross section of the electromagnetic device is divided into $\mathrm{n}$ symmetry sectors, from which only one is modeled using FE and symmetry constraints are set to the periodic boundary.

\subsubsection{Finite element general consideration}

Although finite element is considered the most well-known and the most accurate tool in solving the slow time-varying fields, without some consideration the field solution we may achieve unreliable results. This consideration depends on which value should be calculated from the field domain.

\subsubsection{General consideration in using Lorenz force method}

The Lorentz force method[135] is less sensitive to mesh size than other force calculation methods, pending:

- The skin depth effect is correctly modeled;

- The geometry is appropriately modeled in high current density areas

- The fields are properly modeled in high induced current density areas, which might require higher polynomial order for $J$ in 2D, but especially in 3D.

\subsubsection{General consideration in using Maxwell stress tensor method}


The Maxwell stress method [118], [123] is more sensitive to mesh size than the Lorentz method, however, in cases where the skin depth effect is not accurately modeled, the former will provide better results than the latter.

- Evaluation of large forces on components by the Maxwell stress method is typically accurate. Though, when a small force is anticipated, the magnitude of the force may be at the numerical noise level and hence may be problematic to calculate precisely.

- Air gaps are the most critical parts of the problem to be modeled accurately.

- Multiple layers of elements must be used in the air gaps in order to properly model the large field gradients that might take place there. This can be done through the use of mesh control volumes and virtual air shell.

- Higher polynomial order can help, but multiple layers of elements could prove quite useful even when high order is used.

- All the surrounding air regions that will significantly contribute to the force components of interest should be properly refined, bearing in mind that these regions might not be the closest from the object on which the force is desired.

\subsubsection{General consideration in using energy method In force calculation}

When using the energy method[121], one must be careful as to avoid the cancellation problem in which the force is obtained comes from the subtraction of two energy values differing only in the say the fifth digit of their value. Centered finite differences should give good results with energy values differing from at least the third digit or about $5-10 \%$. In a rotary machine, this often corresponds to a rotation of about 1 degree. If the variance is higher, a new series of simulations should be done with at least twice the number of intermediate positions. 


\subsubsection{Accuracy of cogging torque}

Since cogging torque[126] is usually a small quantity, the model must be accurate enough to ensure that the cogging torque values are above the numerical error level. For this purpose, one cycle of cogging torque (or two cycles to be averaged) should be computed using at least 15 points per cycle and the accuracy should be ensured by:

- Refining the mesh locally in proportion to the magnitude of the $\mathrm{H}$ field. This invariably singles out the air gap area as requiring the greatest mesh density. Air regions inside the motor's inner radius or beyond the motor's outer radius can often be omitted to reduce the number of mesh elements. Using three to four layers in the air gap is very helpful.

\subsection{Thermal modeling}

Heating is a very frequent phenomenon in electromagnetic devices. In many situations, the evaluation of temperature is necessary to avoid over-temperature in structure and electrical insulations. The heat is the main reason for insulation deterioration. There are different sources of heat such as eddy current, conducting current, magnetic hysteresis and mechanical friction. Also there are three different ways that heat is transferred such as the conduction, radiation and convection are these ways.

\subsubsection{Thermal conduction}

Conduction is a process where the heat is transmitted inside a body or between different bodies having physical contact. The basic equation describing thermal conduction is $[129,130]$ :

$$
\text { c. } \frac{d T}{d t}+\nabla \bullet\left(-K_{c} . \nabla T\right)=Q
$$


where $c$ is the thermal capability $\frac{J}{m^{3} \cdot{ }^{o} \mathrm{C}}, K_{c}$ is the thermal conductivity, $\frac{W}{m \cdot{ }^{o} \mathrm{C}}, T$ is the temperature in ${ }^{o} C, Q$ is the thermal source volumetric density $\frac{W}{\mathrm{~m}^{3}}$.

If the $K_{c}$ be independent of the temperature and position, equation (3-83) can be written as:

$$
\nabla^{2} T-\frac{c}{K_{c}} \cdot \frac{d T}{d t}=\frac{Q}{K_{c}}
$$

This equating is similar to the equation (3-7) and can be solved in similar way in one software package.

\subsubsection{Convection transmission[138]}

Convection occurs when a fluid has contact with a heated solid body. There will be a constant movement where the heated particle will be replaced by cooler ones. The main effect is that heat is transmitted from the body to the fluid by following equation.

$$
K_{c} \cdot \frac{d T}{d t} \cdot \vec{n}=-h \cdot\left(T-T_{a}\right)
$$

where $h$ is the coefficient of heat transfer by convection in $\left(\frac{W}{m^{2} \cdot{ }^{o} C}\right), T$ is the temperature at the heated wall (C). $T_{a}$ is the temperature of the fluid at a point far from the wall (C). The quantity $h$ depends on the fluid properties, velocity and geometry. In practical application, $h$ is difficult to be evaluated numerically and it is normally determined experimentally.

\subsubsection{Radiation[138]}

As seen before, normally for the convection and conduction, at least two materials 
must be present in the system. This is not the case for radiation. A body emits electromagnetic waves. This radiation can reach another body. Part of these waves will be reflected and part will be absorbed by second body. This last portion will be transformed into thermal energy.

A body at temperature $T$ radiates energy to another at temperature $T_{a}$, involving it, according to the following expression.

$$
K_{c} \cdot \frac{d T}{d s} \cdot \vec{n}=\varepsilon \cdot \gamma \cdot\left(T^{4}-T_{a}^{4}\right)
$$

where $\gamma$ is the Stefan-Boltzmann constant. And $\varepsilon$ is the emittivity of the body. In electrical machines this term is normally neglected.

\subsubsection{Analytical calculation of thermal PDE equation[139]}

Equation (3-84) represents the general thermal equation of a PMSM. This equation is equivalent to magnetic field equation (3-7) and therfore can be solved in the same finite element domain as equation (3-7). Nevertheless, the wide usage of the embedded thermal model in control, diagnosis and prognostic problems, limit the use of the finite element solutions to the thermal equation to just the design stage. That is why the analytical solution of the thermal equation has received a serious attention. The analytical solution facilitates the use of the lumped parameter methods which is normally represented in sort of T-equivalent circuits shown in figures 3.1 and 3.3. The T-equivalent network represents the equivalent geometry of figure 3.2 and 3.4, respectively. The assumption is that, a PMSM is composed of several of this geometrical element and therefore the overall thermal network can be created by connection of all T-equivalent circuits.

To solve the conductive thermal equation for the figure 3.1 , several assumptions should be made. 
- The heat flows in the different coordinate directions are independent.

- The parameters of the T-equivalent circuit can be realized from the solutions of two boundary value problems. The first boundary value problem has no net external heat flow. The second boundary value problem has a net external heat flow. The superposition of the two solutions then yields the general solution for the temperature field distribution.

In the calculation of the parameters of the T-equivalent circuit in the $\mathrm{X}$ direction for the winding cross-section shown in Figurefigure 3.2, the first boundary value problem is defined as:

$$
\nabla^{2} T=0
$$

where the boundary conditions for the equation (3-87) are defined as $T=T_{0}$ at $A_{0} x$ and $T=T_{1}$ at $A_{1} x$.

The second boundary value problem is defined as:

$$
\nabla^{2} T=-Q / K_{x}
$$

where the boundary conditions for the equation (3-88) are defined as $T=0$ at both $A_{0} x$ and $A_{1} x$. Here, $Q$ is the power loss per unit area and $K_{x}$ the thermal conductivity.

For both boundary value problems it is assumed that there is no heat flow across the boundaries $A_{0} y$ and $A_{1} y$ i.e. $\frac{\partial T}{\partial y}=0$.

The thermal resistances in the T-equivalent network i.e. $R_{1} x, R_{3} x$ and $R_{m} x$ are then calculated, using the solutions of equations (3-87) and (3-88) in the form of:

$$
R_{1} x=\left(T_{1}-T_{m}\right) / f_{1}
$$




$$
\begin{aligned}
& R_{2} x=\left(T_{m}-T_{0}\right) / f_{1} \\
& R_{m} x=\left(\frac{T_{m}^{\prime}}{(Q . S)-R_{1} x}\right) \times\left(\frac{R_{2} x}{R_{1} x+R_{2} x}\right)
\end{aligned}
$$

where $f_{1}$ is the heat flux crossing boundary on $A_{0} x$ (or $A_{1} x$ ) and $T_{m}$ is the mean temperature of the bundle, which can be calculated from:

$$
T_{m}=\frac{1}{S} \int_{s} T(x, y) d x d y
$$

where $S$ is the cross sectional area.

Finally, by using the equations (3-89) to (3-92), the solutions of the (3-87) and (388) for the T-equivalent circuit for the trapezoidal cross-section of figure 3.2 is:

$$
\begin{aligned}
& R_{1} x=R_{2} x=\frac{b-a}{K_{x} \cdot h \cdot L_{s t} \cdot \ln (b / a)} \\
& R_{m} x=\left(\frac{-1}{3}\right) R_{1}
\end{aligned}
$$

Similarly, for the solutions in the Y-direction we have:

$$
\begin{aligned}
& R_{1} y=\frac{h}{2 K_{x} \cdot L_{s t} \cdot(b-a)}\left(1-\frac{2 a^{2} \ln (b / a)}{\left(b^{2}-a^{2}\right)}\right) \\
& R_{2} y=\frac{h}{2 K_{x} \cdot L_{s t} \cdot(b-a)}\left(\frac{2 b^{2} \ln (b / a)}{\left(b^{2}-a^{2}\right)}-1\right) \\
& R_{m} y \approx\left(\frac{-1}{6}\right)\left(R_{1} y+R_{2} y\right)
\end{aligned}
$$

The total thermal capacitance of the cylinder is independent from thermal Poisson equation and can be expressed in form of material weight density $\rho$ and specific heat capacity $c_{p}$ as:

$$
C=\frac{\rho \cdot c_{p} \cdot L_{s t} \cdot h \cdot(a+b)}{2}
$$


For conductor bundles having more complex cross-sections, numerical methods might be needed to calculate the mean temperature and therefore the circuit parameters.

The equations (3-87) and (3-88) can be similarly solved for a general cylindrical element. The reason for usage of the cylindrical element is that, the solid components of a PMSM are better matched with a general cylindrical component shown in figure 3.4. To obtain simple, but physically significant, expressions for the network of thermal resistances that describe the heat conduction across the general component, the following assumptions are made

- The heat flow in the radial and axial directions is independent.

- A single mean temperature defines the heat flow both in the radial and axial directions.

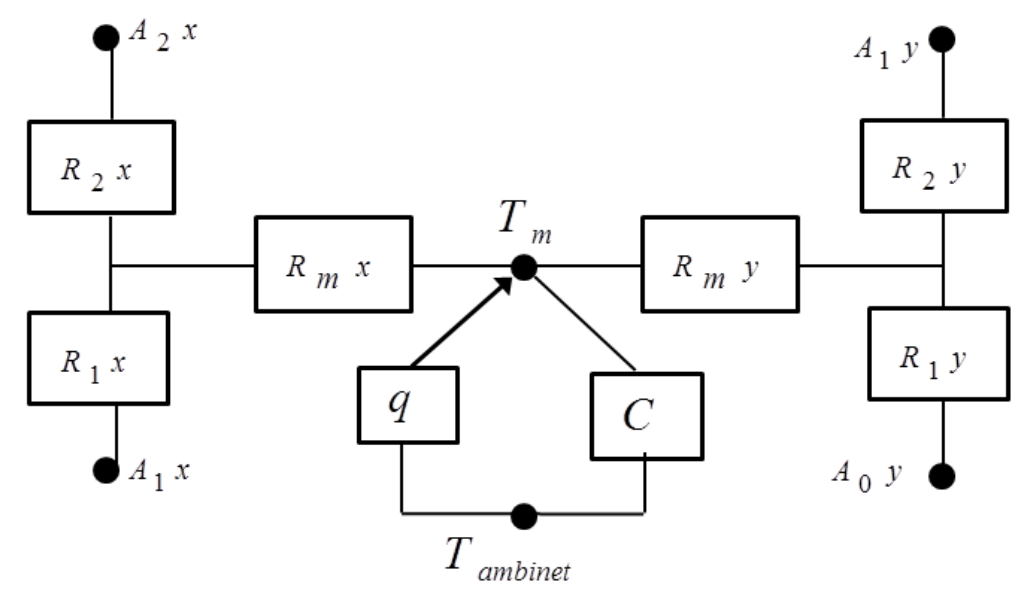

Figure 3.1 T-equivalent thermal network for trapezoidal geometrical element 


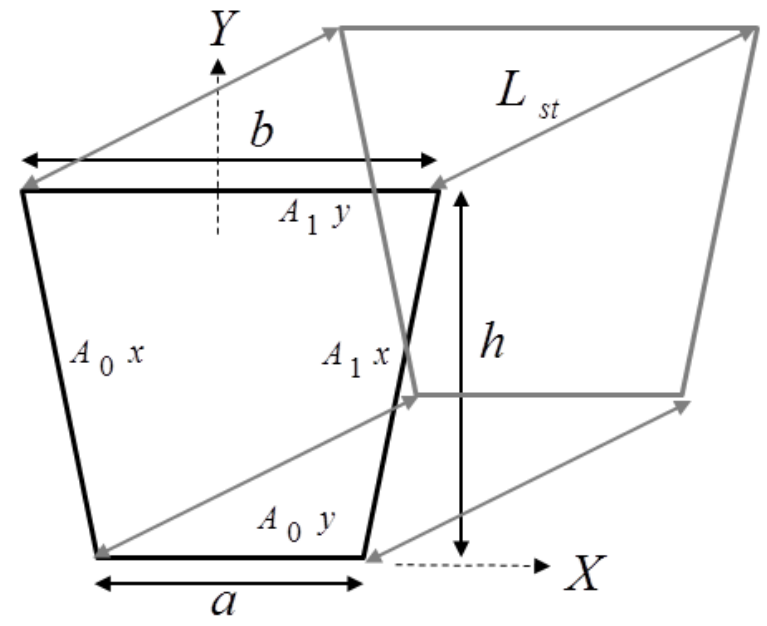

Figure 3.2 Trapezoidal geometrical element of a PMSM

- There is no axial heat flow. The thermal capacity and heat generation are uniformly distributed.

The solution for the arc segment cross-section shown in figure 3.4 is again a Tequivalent circuit, figure 3.3. It can be shown that the thermal resistances in the circumferential direction can be represented as:

$$
\begin{aligned}
& R_{1} \theta=R_{2} \theta=\frac{\alpha}{2 \cdot L_{s t} \cdot K_{\theta} \cdot \ln \left(r_{o} / r_{i}\right)} \\
& R_{m} \theta=\frac{-\alpha}{6 \cdot L_{s t} \cdot K_{\theta} \cdot \ln \left(r_{o} / r_{i}\right)}
\end{aligned}
$$

where $K_{\theta}$ is the average thermal conductivity and $L_{s t}$ the axial length of the conductor bundle. Similar analytical expressions can be derived for radial thermal parameters as:

$$
R_{1} r=\frac{1}{2 \cdot \alpha \cdot L_{s t} \cdot K_{r}}\left[1-\frac{2 r_{o}^{2} \ln \left(\frac{r_{o}}{r_{i}}\right)}{r_{o}^{2}-r_{i}^{2}}\right]
$$




$$
\begin{aligned}
& R_{2} r=\frac{1}{2 \cdot \alpha \cdot L_{s t} \cdot K_{r}}\left[\frac{2 r_{1}^{2} \ln \left(\frac{r_{o}}{r_{i}}\right)}{r_{o}^{2}-r_{i}^{2}}-1\right] \\
& R_{m} r=\frac{-1}{4 \cdot \alpha \cdot\left(r_{o}^{2}-r_{i}^{2}\right) L_{s t} \cdot K_{r}}\left[r_{i}^{2}+r_{o}^{2}-\frac{4 \cdot r_{1}^{2} \cdot r_{2}^{2} \ln \left(\frac{r_{o}}{r_{i}}\right)}{r_{o}^{2}-r_{i}^{2}}\right]
\end{aligned}
$$

The thermal capacitance can be expressed in form of material weight density $\rho$ and specific heat capacity $c_{p}$ as:

$$
C=\frac{\alpha \cdot \rho \cdot c_{p} \cdot \pi \cdot L_{s t} \cdot\left(r_{o}^{2}-r_{i}^{2}\right)}{2}
$$

In using these assumptions, the solution of the heat conduction equations in each of the axial and radial directions produces two separate three-terminal networks. In each network, two of the terminals have two one-dimensional networks are combined simply by connecting the points of mean temperature together.

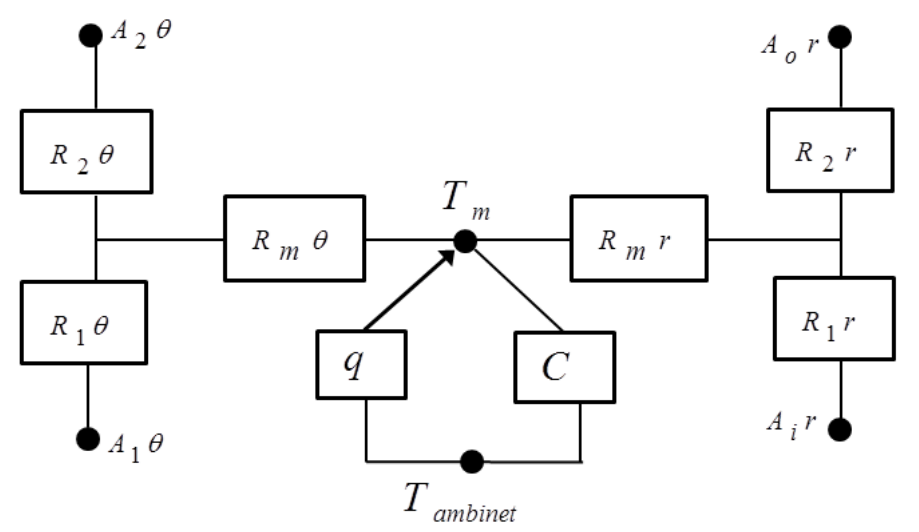

Figure 3.3 T-equivalent thermal network for cylindrical geometrical element 


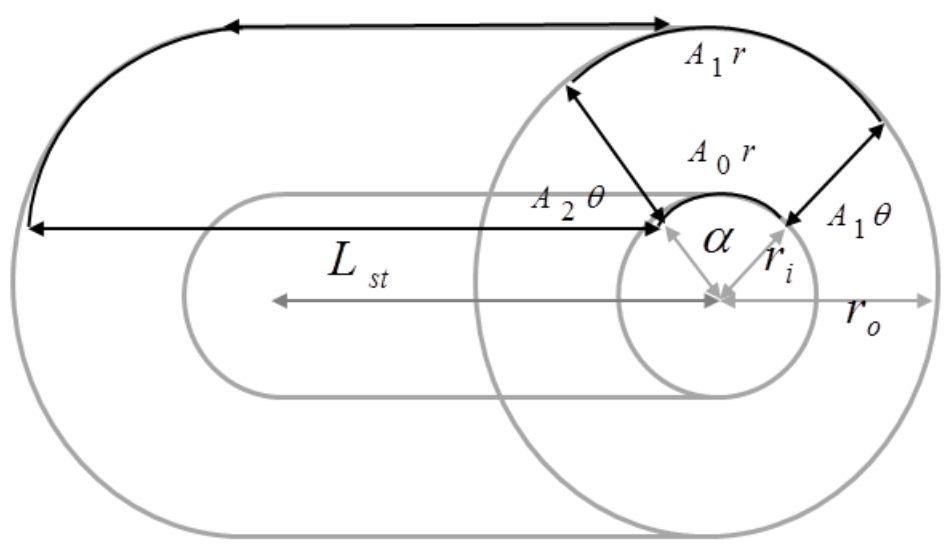

Figure 3.4 Cylindrical geometrical element of a PMSM

\subsubsection{Simplification conductive thermal resistance}

The numerical and experimental result show that the major part of the conductive heat transfer is through the radial direction. This helps to neglect the circumferential thermal resistances. The solution of equations (3-87) and (3-88) neglecting the circumferential term lead to a single thermal resistance. In this new formulation, it is assumed that the heat source is in the radial direction from $A_{i} r$ to $A_{o} r$. Also, the entire heat source is collected on the face $A_{i} r$. This means that the mean temperature is assumed to be:

$$
T_{m}=\left(T_{i} r+T_{o} r\right) / 2
$$

where $T_{i} r$ is the boundary temperature at $A_{i} r$ and $T_{i} r$ is the boundary temperature at $A_{o} r$

For the cylindrical element the simplified T-equivalent model is shown in figure 3.5. It can be shown that the single conductive thermal resistance can be calculated as:

$$
R=\left(R_{1} r+R_{2} r\right)+R_{m} r=\frac{\ln \left(r_{o} / r_{i}\right)}{\alpha \cdot K_{r} \cdot L_{s t}}
$$




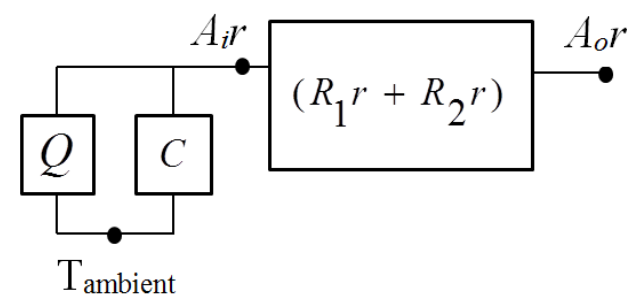

Figure 3.5 Simplified thermal element for the cylindrical geometrical element

\subsubsection{Complete thermal model of a PMSM}

The basis of the used thermal model, figure 3.3, is presented at[23], [138]. The complete model of the machine model consists of a network of twelve thermal resistances and seven thermal capacitances that make it possible to estimate the temperatures of magnets, copper and more even critical points inside the machine, accurately. The inputs to the thermal network are the active losses of the windings and the core losses equaled as the current source in thermal network and the out puts are the temperatures measured equaled to the voltages from the thermal network. The air gap resistance is empirically suggested in a form of:

$$
R_{a g}=\frac{g}{N_{n u} \cdot K_{a i r} \cdot A_{g}} \quad[W / K]
$$

where $R_{a g}$ is the air gap thermal resistance, $N_{n u}$ is the Nusselt number, $A_{g}$ is the air gap area, $g$ is the air gap length and $K_{\text {air }}$. is the thermal conductivity of the air gap.

The $R_{r s}, R_{m r}, R_{s h}, R_{s t}, R_{s y}, R_{u s}, R_{\text {case }}$ and $R_{\text {endcap }}$ are the conductive thermal resistances of the rotor core, magnets, shaft stator teeth, stator yoke, casing and the ending cap, respectively. These resistances are calculated based on the assumption that the heat transfer for a cylindrical element is done only in radial direction. These resistances are calculated from equation (3-108).

It is mentioned that before calculation of the conductive resistances, the equivalent 
geometry of the machine in which all the element parts were aligned in the radial direction in the form of cylindrical slices should be calculated. In reference [140], a good example of this kind of geometry conversion is presented.

$R_{\text {ambendcap }}$ and $R_{\text {amb }}$ are the respective convective resistance of the ending caps and the casing to the ambient temperature, respectively. The solution of the equation $(3-87)$ is expressed in form of:

$$
R_{\text {convective }}=\frac{1}{h_{\text {cnd }} \cdot A_{\text {cnd }}} \quad[\mathrm{W} / \mathrm{K}]
$$

where $h_{c n d}$ is the convection coefficient and the $A_{c n d}$ is the contact surface of the ending caps and the casing to the ambient.

The thermal capacitances of all elements can be given by equations (3-104). The resistive losses of the stator winding can be defined as:

$$
P_{\text {winding }}=\sum_{i=1}^{3} R_{i} \cdot I_{i}^{2}[W]
$$

where $R_{i}$ is the winding resistance at a given temperature and $I_{i}$ is the RMS value of the current for each phase.

Stator iron losses can be divided in losses due to hysteresis, $P_{h}$ and the eddy currents, $P_{e}$. The total stator iron losses as well as the hysteresis and eddy current losses are calculated by:

$$
P_{\text {core }}=k_{h} \cdot B_{\max }^{2} \cdot f+k_{e} \cdot B_{\max }^{2} \cdot f^{2}[W]
$$

where $B_{\max }$ represents the maximum magnetic flux density in the iron part of PMSM and it is calculated from the static FE analysis of machine under nominal current, $f$ is the electrical frequency, $k_{h}$ and $k_{e}$ are empirically derived parameters.

In inter-turn short circuit fault (ISCF) studies, for simulation of the shorted turn's 
temperature and its neighbor's turns, one needs to divide the machine windings into healthy phases and faulty phase. The faulty phase is divided into the three parts of the healthy turns non-adjacent to the faulty turns (BNA), healthy turns adjacent to faultyturns (BA) and faulty turns (FB). The schematic view of the thermal model of the winding in a faulty machine is depicted in figure 3.7. In this figure phases " $a$ " and "c" are healthy and phase "b" is faulty phase. Also in figure 2(b), the accumulative heat of phases "a" and "c" is $P_{H A C}, P_{H B N A}, P_{H B A}$ and $P_{F B}$ show the input power to the model from different sections of phase "b". $R_{w a}, R_{w a 1}, R_{w a 2}$ and $R_{w a 3}$, respectively indicate the end winding convective resistances of phases "a" and "c" together, phase "b" nonadjacent part, phase "b" adjacent part and faulty part of "b". These are calculated from equation (3-113) to (3-117). The respective conductive thermal resistances to these parts i.e. $R_{u s}, R_{w s h n}, R_{\text {wsh }}$ and $R_{w s f a}$ are calculated by equation (3-108).

$$
\begin{aligned}
& R w a_{0}=\frac{1}{15.5 \times S w a \times((0.29 \times v)+1))} \\
& R w a=\frac{3 \times R w a_{0}}{2} \\
& R w a_{1}=\frac{3 \times R w a_{0} \times N o s}{N o s-6} \\
& R w a_{2}=\frac{3 \times R w a_{0} \times\left(\frac{N o s}{6}\right)+\left(N_{f}+N_{s}\right)}{N_{s}} \\
& R w a_{3}=\frac{3 \times R w a_{0} \times\left(\frac{N o s}{6}\right)+\left(N_{f}+N_{s}\right)}{N_{f}}
\end{aligned}
$$

where Nos is the number of slots, $N_{s}$ is the number of healthy turns per coil and $N_{f}$ is number of faulty turns in faulty coil. Swa is the total area of end winding in 
adjacent to air. $v$ is the speed of inner air.

Here, Rhf is the conductive resistance between faulty turns and healthy turns in neighboring turns. This is calculated from equation (3-108) based on the equivalent assumption that a uniform electrical insulation separated this two parts radially.

\subsubsection{Solution of the thermal network [139]}

The choice of the proper geometrical element and the number of geometrical element in a PMSM depends on the machine shape. However, after decomposition of the machine geometry to the trapezoidal or cylindrical element the complete thermal network of the PMSM will be established from the connection of all T-equivalent networks. The individual node temperatures in T-equivalent network are defined by a set of heat balance equations in the form of:

$$
C_{i} \frac{d T}{d t}=\frac{1}{R_{i j}}\left(T_{j}-T_{i}\right)+Q_{i} i, j=1, \ldots, n
$$

where $C_{i}$ is node thermal capacitance, $T_{i}$ is the temperature at node $i \cdot R_{i j}$ is the is thermal resistance between adjoining nodes $i$ and $j, Q_{i}$ is the heat generation at node $i$.

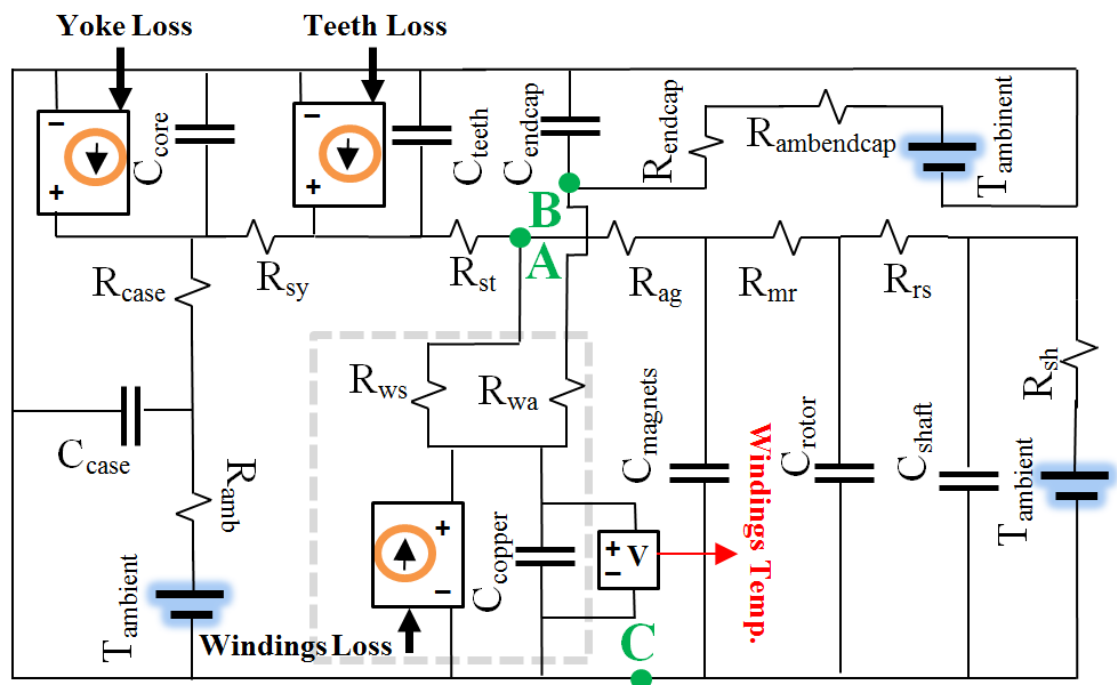


Figure 3.6 Healthy Transient thermal model of the machine

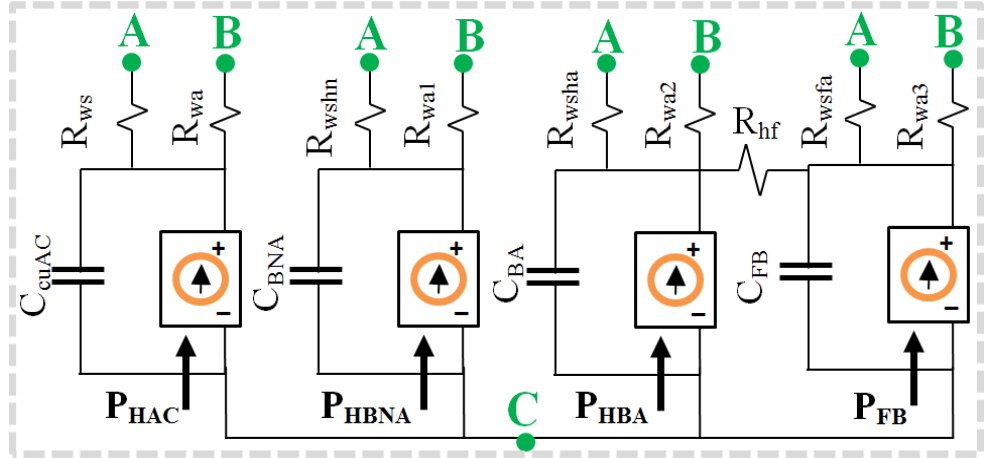

Figure 3.7 Modified transient thermal model of the windings for ISCF studies

In the final thermal network some nodes have no thermal capacitance and heat generation associated with them. On eliminating these mathematically, the system can be reduced to linear differential equations, expressed in matrix form as

$$
[C] \frac{d[T]}{d t}=\left[Y_{r}\right][T]+[Q] i, j=1, \ldots, n
$$

where $[C]$ is the column matrix of thermal capacitance; $\left[Y_{r}\right]$ is a square matrix of inter-nodal conductance; $[Q]$ is a column matrix of thermal generators.

\subsection{Modeling of demagnetization of magnets}

- $\quad$ Thermal demagnetization[22]

In general, in life assessment studies of PM materials the relation of temperature and PM loss can be categorized in three classes of reversible losses, irreversible but recoverable losses and irreversible and unrecoverable losses. The reversible loss is defined for all the operating points above the knee point. It is the loss that is recovered when the magnet returns to its original temperature. Irreversible but recoverable losses are defined as partial demagnetization of the magnet from exposure to high or low temperatures. These losses are only recoverable by re-magnetization and are not recovered when the temperature returns to its original value. These losses occur when 
the operating point of the magnet falls below the knee of the demagnetization curve. Irreversible and unrecoverable losses occur in magnets exposed to very high temperatures and are not recoverable by re-magnetization. Here, for physical modeling both the reversible and irreversible loss is considered. As shown in figure 3.8 , a change in temperature causes the working point to shift on the working line from $\mathrm{P}_{1}$ to $\mathrm{P}_{2}$. As long as the working point stays above the knee point, the changes in flux density are reversible, i.e. after cooling the flux density returns to its original value. In all other cases any change in flux density is irreversible (irreversible magnetic losses).

\section{- Reverse field demagnetization[22]}

External magnetic fields in repulsion modes will lead to demagnetizing effect on permanent magnets. As shown in figure 3.8, when operating point moves from P1 to $\mathrm{P} 2$ due to a strong reverse magnetic field, the remnant flux density $\mathrm{B}_{\mathrm{r}}$ is decreased to $\mathrm{B}_{\mathrm{r}}$ and irreversible demagnetization occurs. When the strong reverse field is removed, the new operating point is then established at P3 instead of P1.

Figure 3.9 shows a typical demagnetization curves for sintered magnet on a $\mathrm{Nd}$ Fe-B base was used here. In order to have a closed form for this data, it is preferred to interpolate this surface in the form of:

$$
H=p_{00}+p_{10} \cdot T+p_{01} \cdot B_{r}+p_{11} \cdot T \cdot B_{r}+p_{02} \cdot B_{r}^{2}
$$

where $B_{r}$ is the remnant flux density, $T$ is the temperature and $H$ is normal flux intensity. A typical sintered magnet on a Nd-Fe-B, $\left[p_{00}, p_{10} . p_{01}, p_{11}, p_{02}\right]$ can be $[-$

$$
1218.4,7.5,148.6,-4,535]
$$

From figure 3.8 it is obvious that the average remnant flux density can be used to define the operating point of the magnet. The resultant flux density on the face of PM 
has two parts. The first part belong to the armature windings direct reverse flux which is perpendicular on the magnet face, $\bar{B}_{a r}$. The other part belongs to initial PM remnant flux density $\bar{B}_{m r}$. The resultant normal magnetic flux density $\bar{B}_{r r}$ is simply calculated by summation of these two as:

$$
\bar{B}_{r r}=\bar{B}_{m r}+\bar{B}_{a r}+\frac{1}{\tau_{m} s+1} \times\left(\min \left(D_{r i s k}\right) \mid \min \left(D_{r i s k}\right)<0\right)
$$

where $\bar{B}_{m r}(\theta)$ is calculated from a transient FE analysis with motion of the PMSM for zero armature reaction at standard room temperature of $20^{\circ}(\mathrm{C})$ for full electrical rotation. $\bar{B}_{m r}(\theta)$ is calculated in the outer face of cubic magnet. This face is perpendicular to the direction of magnetic flux path. The conditional memory function $\left(\min \left(Q_{\text {risk }}\right) \mid \min \left(Q_{\text {risk }}\right)<0\right)$ is a negative value that indicates the minimum value of all past resultant magnet flux density on the face of PM experienced below the knee point. This memory function is activated when $D_{\text {risk }}$ falls below zero. This interprets the physics of irreversible demagnetization as explained in figure 3.8.b. In last section $n$ of this chapter the calculation of the $\bar{B}_{a r}$ and $D_{\text {risk }}$ is explained. $\tau_{m}$ is the time constant of PM demagnetization and " $s "$ is the Laplace operator.

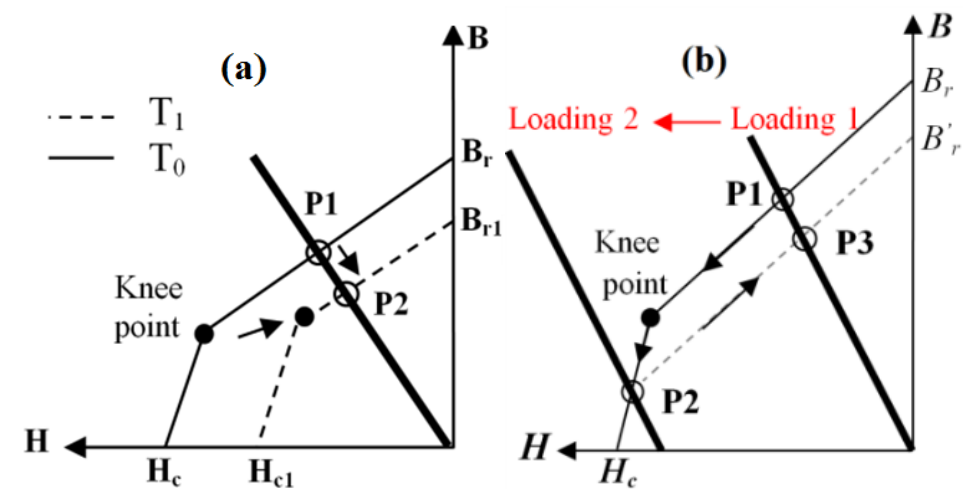


Figure 3.8 Reversible demagnetization process of a typical sintered magnet on a Nd-Fe-B base at two temperatures $(\mathrm{T} 1>\mathrm{T} 0)$ (a), irreversible demagnetization due to direct reverse field (b)

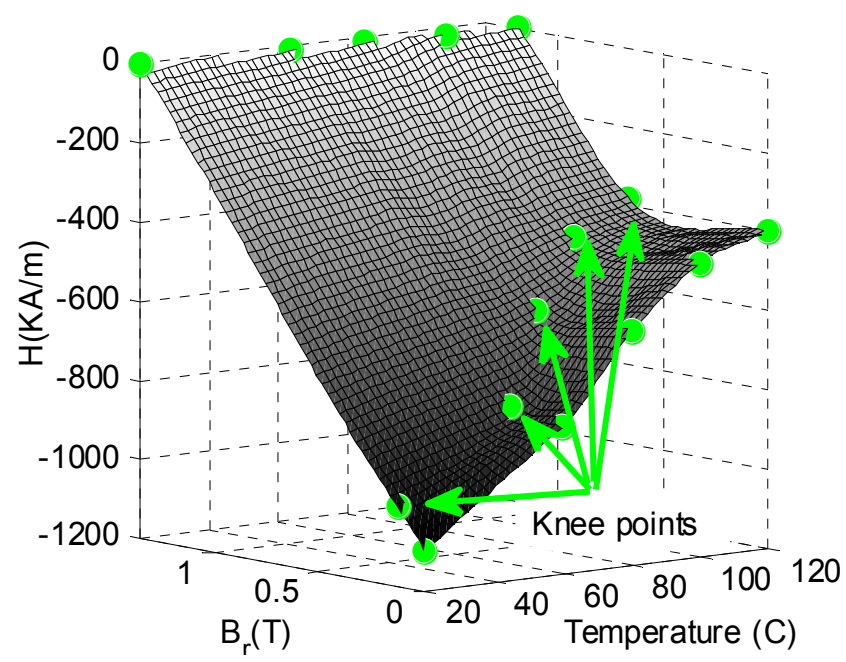

Figure 3.9 Typical demagnetization surface for sintered magnet on a Nd-Fe-B base, $\mathrm{B}(\mathrm{H})$ at different temperatures [4]

\subsection{Analytical voltage equation of healthy PMSM[83]}

A 2-pole, PMSM is illustrated in figure 3.10. The 3-phase, wye-connected stator winding and permanent magnet motor are shown in figure 3.11. It is a synchronous machine. The stator winding are identical windings displaced $120^{\circ}$, each with equivalent $N_{s \text { turns and resistance }} r_{s}$. For our analysis we will assume that the stator winding are sinusoidal distributed. The three sensors presented in the figure are the Hall Effect sensors. When the North Pole is under the sensor, its output is non-zero; with South Pole under the sensor the output is zero. In many applications the sensor is supplied from an inverter that is switched at a frequency corresponding to the rotor speed. The state of the three sensors is used to define the switching logic for the inverter. 


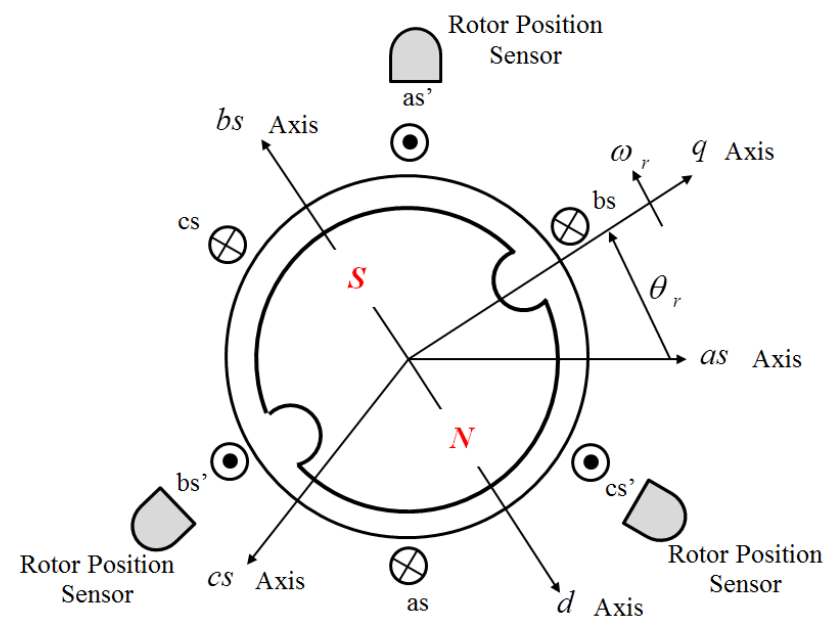

Figure 3.10 Schematic view of a 2-pole, PMSM

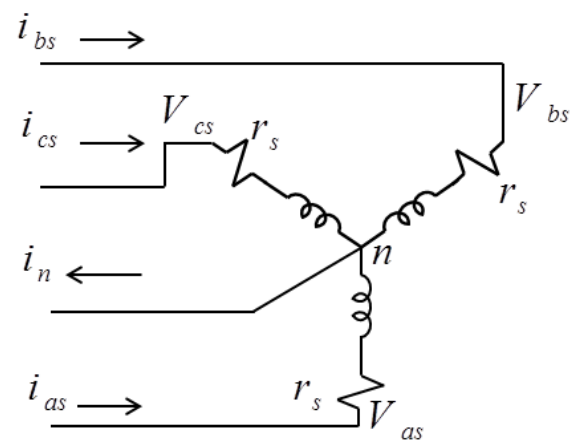

Figure 3.11 3-phase balance winding of a PMSM

In a real machine, the sensors are not positioned over the rotor as shown in the figure. Instead, they are located over a ring that is attached on the shaft external to the stator windings and magnetized as the rotor. It is essential to start the voltage and the torque equations that can be used to define the behavior of the permanent magnet synchronous machine. The voltage equation in machine variables is:

$$
V_{a b c s}=r_{s} \cdot i_{a b c s}+p \lambda_{a b c s}
$$

where the $r_{s}$ and the term $\lambda_{a b c s}$ look like:

$$
r_{s}=\operatorname{diag}\left[r_{s}, r_{s}, r_{s}\right]
$$


The flux linkage might be written as

$$
\lambda_{a b c s}=L_{s} \cdot i_{a b c s}+\lambda_{s}^{\prime \prime}
$$

where $L_{s}$ is the $3 \times 3$ inductance matrix that may be written in the form of:

$$
L_{s}=\left[\begin{array}{ccc}
L_{l s}+L_{A}-L_{B} \cdot \cos 2 \theta_{r} & \frac{-1}{2} L_{A}-L_{B} \cos 2\left(\theta_{r}-\frac{\pi}{3}\right) & \frac{-1}{2} L_{A}-L_{B} \cos 2\left(\theta_{r}+\frac{\pi}{3}\right) \\
\frac{-1}{2} L_{A}-L_{B} \cos 2\left(\theta_{r}-\frac{\pi}{3}\right) & L_{l s}+L_{A}-L_{B} \cdot \cos 2\left(\theta_{r}-\frac{2 \pi}{3}\right) & \frac{-1}{2} L_{A}-L_{B} \cos 2\left(\theta_{r}+\pi\right) \\
\frac{-1}{2} L_{A}-L_{B} \cos 2\left(\theta_{r}+\frac{\pi}{3}\right) & \frac{-1}{2} L_{A}-L_{B} \cos 2\left(\theta_{r}+\pi\right) & L_{l s}+L_{A}-L_{B} \cdot \cos 2\left(\theta_{r}+\frac{2 \pi}{3}\right)
\end{array}\right]
$$

Also

$$
\lambda_{m}^{\prime \prime}=\lambda_{m}^{\prime}\left[\begin{array}{c}
\sin \left(\theta_{r}\right) \\
\sin \left(\theta_{r}-\frac{2 \pi}{3}\right) \\
i n\left(\theta_{r}+\frac{2 \pi}{3}\right)
\end{array}\right]
$$

where $\lambda_{m}^{\prime}$ is the amplitude of the flux linkage established by the permanent magnet as viewed from the stator windings. In other words, the magnitude of the $p \lambda_{m}^{\prime \prime}$ will be the open circuit voltage induced in each stator phase voltage.

The PMSM has the physical look of a 3-phase synchronous machine that is supplied from an inverter that converts a DC voltage to 3-phase alternative-voltage (ac) with frequency corresponding instantaneous to rotor speed. The electromagnetic torque in $a-b-c$ frame of reference is defined as:

$$
\begin{gathered}
T_{e}=\left(\frac{P}{2}\right)\left\{\frac { ( L _ { m d } - L _ { m q } ) } { 3 } \left[\left(i_{a s}^{2}-\frac{i_{b s}^{2}}{2}-\frac{i_{c s}^{2}}{2}-i_{a s} \cdot i_{b s}-i_{a s} \cdot i_{c s}+2 i_{b s} \cdot i_{c s}\right) \sin 2 \theta_{r}\right.\right. \\
\left.\left.+\frac{\sqrt{3}}{2}\left(i_{b s}^{2} \cdot i_{c s}^{2}-2 i_{a s} i_{b s}+2 i_{a s} i_{c s}\right) \cdot \cos 2 \theta_{r}\right]+\lambda_{m}^{\prime}\left[\left(i_{a s}-\frac{i_{b s}}{2}-\frac{i_{c s}}{2}\right) \cdot \cos \theta_{r}+\frac{\sqrt{3}}{2}\left(i_{b s}-i_{c s}\right) \sin \theta_{r}\right]\right\}
\end{gathered}
$$


where the magnetizing inductances are defined as

$$
\begin{aligned}
& L_{m d}=\frac{3 \times\left(L_{A}-L_{B}\right)}{2} \\
& L_{m d}=\frac{3 \times\left(L_{A}+L_{B}\right)}{2}
\end{aligned}
$$

The mechanical system is defined as:

$$
\begin{aligned}
& T_{l}-T_{e}=\left(\frac{2}{P}\right) \times\left[J \frac{d \omega_{r}}{d t}+B . \omega_{r}\right] \\
& \omega_{r}=\frac{d \theta_{r}}{d t}
\end{aligned}
$$

The $\theta_{r}$ is the electrical rotor position. $\omega_{r}$ is the electrical angular velocity $(\mathrm{rad} / \mathrm{s})$. $J$ is the inertia of the rotor $\mathrm{Kg} \cdot \mathrm{m}^{2}$ and the connected load to it. $B$ is the friction coefficient N.m.s.

\section{7 d-q transform[83]}

The usage of the rotor frame of reference $\left(\mathrm{dq}_{\mathrm{q}} \mathrm{s}^{\mathrm{r}}\right)$ allows the familiarity with salient operating features of inverter-motor combination without becoming associated with harmonics of the phase voltage that occur due to the switching of the inverter. A change of variables that formulates a transformation of 3-phase variables of stationary circuit elements to the arbitrary reference frame may be expressed as:

$$
f_{q d 0}=K_{s} \cdot f_{a b c s}
$$

where

$$
\left(f_{q d 0 s}\right)^{T}=\left[f_{q s}, f_{d s}, f_{0 s}\right]
$$




$$
K_{s}=\frac{2}{3}\left[\begin{array}{ccc}
\cos (\theta) & \cos \left(\theta-\frac{2 \pi}{3}\right) & \cos \left(\theta+\frac{2 \pi}{3}\right) \\
\sin (\theta) & \cos \left(\theta-\frac{2 \pi}{3}\right) & \cos \left(\theta+\frac{2 \pi}{3}\right) \\
1 & 1 & 1
\end{array}\right]
$$

It can be shown that for the inverse transformation we can have:

$$
K_{s}=\frac{2}{3}\left[\begin{array}{ccc}
\cos (\theta) & \sin (\theta) & 1 \\
\cos \left(\theta-\frac{2 \pi}{3}\right) & \sin \left(\theta-\frac{2 \pi}{3}\right) & 1 \\
\cos \left(\theta+\frac{2 \pi}{3}\right) & \sin \left(\theta+\frac{2 \pi}{3}\right) & 1
\end{array}\right]
$$

The angular velocity, $\omega$ and the angular displacement $\theta$, of the arbitrary reference frame are related by:

$$
\theta=\int_{0}^{t} \omega(\xi) \cdot d \xi+\theta(0)
$$

where $\xi$ is a dummy variable of integration. The term $f$ in (3-133) can represent either voltage, current, flux linkage or electric change. The subscript $T$ denotes the transpose of the matrix. The subscript ' $s$ ' is the stationary frame of the reference. 


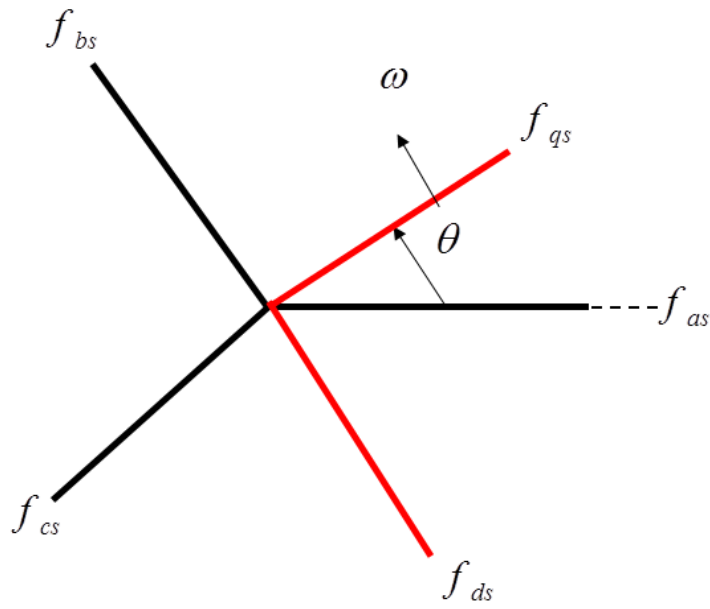

Figure 3.12 Phasor transformation of 3-phase balance a-b-c frame to d-q frame of reference

\subsubsection{Transformation between reference frames [83]}

In some derivations and analyses, it is convenient to relate variables in one reference frame to variables in another reference frame directly, without involving the a-b-c variables in the transformation. In order to start this transformation between any two frames of reference, let " $x$ " denote the reference frame from which the variables are being transformed and let "y" denote the reference frame to which the variable are being transformed.

$$
f_{q d 0}^{y}={ }^{x} K^{y} \cdot f_{q d 0 s}^{x}
$$

Also, at the same time the reverse transformation is valid.

$$
f_{q d 0}^{x}={ }^{y} K^{x} \cdot f_{q d 0 s}^{y}
$$

The transformation ${ }^{x} K^{y}$ is simply calculated as:

$$
{ }^{x} K^{y}=K_{s}^{y} \cdot\left(K_{s}^{x}\right)^{-1}=\left[\begin{array}{ccc}
\cos \left(\theta_{y}-\theta_{x}\right) & -\sin \left(\theta_{y}-\theta_{x}\right) & 0 \\
\sin \left(\theta_{y}-\theta_{x}\right) & \cos \left(\theta_{y}-\theta_{x}\right) & 0 \\
0 & 0 & 1
\end{array}\right]
$$




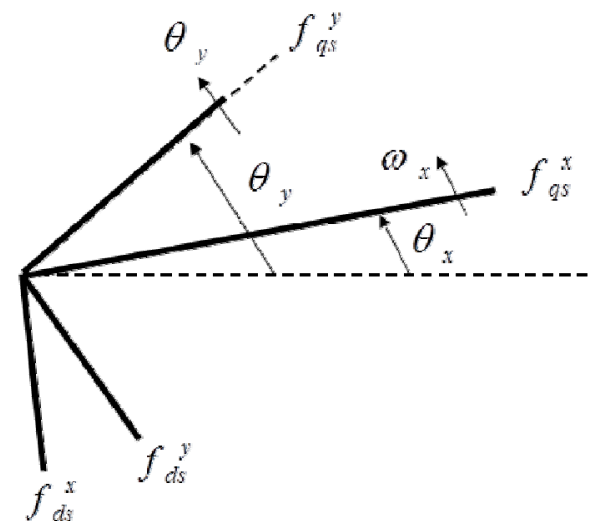

Figure 3.13 Transformation between two $d q 0 s^{x}$ and $d q 0 s^{y}$ frame of reference

\section{8 d-q model of healthy PMSM[83]}

By applying the equations (3-132) to (3-136) to equations (3-121) to (3-126), the machine voltage equation is derived as:

$$
\begin{aligned}
& V_{q d 0 s}^{r}=r_{s} \cdot i_{q d 0 s}^{r}+\omega_{r} \cdot \lambda_{q d s}^{r}+p \lambda_{q d 0 s}^{r} \\
& \left(\lambda_{q d s}^{r}\right)^{T}=\left[\lambda_{d s}^{r},-\lambda_{q s}^{r}, 0\right] \\
& \lambda_{q d 0 s}^{r}=\left[\begin{array}{ccc}
L_{l s}+L_{m q} & 0 & 0 \\
0 & L_{l s}+L_{m d} & 0 \\
0 & 0 & L_{l s}
\end{array}\right] \cdot\left[\begin{array}{c}
i_{q s}^{r} \\
i_{d s}^{r} \\
i_{0 s}^{r}
\end{array}\right]+\lambda_{m}^{r}\left[\begin{array}{l}
0 \\
1 \\
0
\end{array}\right]
\end{aligned}
$$

The subscript " $r$ " denotes the rotor frame of reference.

By more simplifications, the voltage equation in rotor frame of reference may be written directly as:

$$
\begin{aligned}
& V_{q s}^{r}=r_{s} \cdot i_{q s}^{r}+\omega_{r} \cdot \lambda_{d s}^{r}+p \lambda_{q s}^{r} \\
& V_{d s}^{r}=r_{s} \cdot i_{d s}^{r}-\omega_{r} \cdot \lambda_{q s}^{r}+p \lambda_{d s}^{r} \\
& V_{0}=r_{s} \cdot i_{0 s}+p \lambda_{0 s}
\end{aligned}
$$

where

$$
\lambda_{q s}^{r}=L_{q} \cdot i_{q s}^{r}
$$




$$
\begin{aligned}
& \lambda_{d s}^{r}=L_{d} \cdot i_{d s}^{r}+\lambda_{m}^{r} \\
& \lambda_{0 s}=L_{l s} \cdot i_{0 s}
\end{aligned}
$$

where

$$
\begin{aligned}
& L_{q}=L_{l s}+L_{m q} \\
& L_{d}=L_{l s}+L_{m d}
\end{aligned}
$$

Because $p \lambda_{m}^{\prime r}=0$ we can have:

$$
\begin{aligned}
& V_{q s}^{r}=\left(r_{s}+p L_{q}\right) i_{q s}^{r}+\omega_{r} L_{d} i_{d s}^{r}+\omega_{r} \lambda_{m}^{r} \\
& V_{d s}^{r}=\left(r_{s}+p L_{d}\right) i_{d s}^{r}-\omega_{r} L_{q} i_{q s}^{r} \\
& V_{0 s}=\left(r_{s}+p L_{l s}\right) i_{0 s}
\end{aligned}
$$

The expression for the electromagnetic torque may be written as:

$$
T_{e}=\left(\frac{3}{2}\right)\left(\frac{P}{2}\right)\left(\lambda_{m}^{r} . i_{q}^{r}+\left(L_{d}-L_{q}\right) i_{q s}^{r} i_{d s}^{r}\right)
$$

As mentioned in the previous section, the state of the sensor provides information regarding the position of the poles and thus the position of the " $d$ " and " $q$ " axes . In other words, when the machine is supplied from an inverter, it is possible, by controlling the firing on the inverter, to change the values of $V_{q s}^{r}$, and $V_{d s}^{r}$. Recall that $\theta_{r}$ in the transformation equation to the rotor reference frame can be written as:

$$
\omega_{r}=\frac{d \theta_{r}}{d t}
$$

For purpose of discussion, let us assume that the angle of the three phase applied stator voltage is $\theta_{e v}$. When the machine is supplied from an inverter, the stator voltage will have a stepped waveform. Nevertheless, the above voltages may be considered as the fundamental components of these stepped phase voltage. Also 


$$
\omega_{e}=\frac{d \theta_{e v}}{d t}
$$

The PMSM is, by definition, a device where the frequency of fundamental component of the applied stator voltages corresponds to the speed of the rotor and we understand this is accomplished by firing the inverter supplying the machine. Therefore in PMSM $\omega_{e}=\omega_{r}$. If the voltage equations are substituted into $K_{s}^{r}$ we obtain.

$$
\begin{aligned}
& V_{q s}^{* r}=\sqrt{2} V_{s} \cos \phi_{v} \\
& V_{d s}^{* r}=-\sqrt{2} V_{s} \sin \phi_{v}
\end{aligned}
$$

where

$$
\phi_{v}=\theta_{e v}-\theta_{r}
$$

We can adjust $\phi_{v}$ by appropriate firing of the inverter, thereby changing the phase relationship between the fundamental component of the 3-phase stator voltage and rotor permanent magnet axis. In most applications, however, $\phi_{v}$ is fixed to zero so that as far as the fundamental component is concerned $V_{d}^{r}=0$ and $V_{q}^{r}=\sqrt{2} V_{s}$.

\subsection{Healthy phase variable motor model in a-b-c frame of reference[141-148]}

The increase in switching frequencies of modern inverters has led to a growth in the power densities of machines with a corresponding decrease in the size of the circuitry. The physical modeling of the machines is playing a serious role in the design of an integrated drive system. To solve the limitations of analytical designs as well as standard d-q modeling, a low frequency physical phase variable motor model was developed by Mohammed et al. [141-148]. The phase variable machine model is based on three-phase axis theory instead of two phases that are present in the d-q 
models, however the line-line modeling approach reduce the size of the modeling problem from usage of $3 \times 3$ matrices to $2 \times 2$ ones. The model can forecast the low frequency performance such as torque pulsations, current ripples but cannot predict the high frequency effects such as motor current spikes, ground currents and inverter induced overvoltage. To predict these effects, the machine model should reflect not only the material nonlinearity as well as geometrical and slotting effects but also the effects of inter-winding capacitances, conductor types, distributed effects, winding and insulator configuration and material characteristics.

As stated earlier, d-q modeling of the machines is not satisfactory as it completely ignores harmonics in the machine operational parameters. It assumes sinusoidal distribution of air gap flux density and obviously all the derived waveforms from it. Furthermore, the d-q model becomes mathematically inaccurate when the symmetry of mutual inductances gets removed in some specific design applications[148]. Also, during the transient conditions, when the stator and rotor mmfs are not constant in magnitude or in angle with respect to each other, then the harmonic components are generated in the electrical and mechanical system. These harmonic components cannot be described precisely using d-q model. Hence, a new modeling method was presented called the physical phase variable model to symbolize the machine in threephase reference frame (a-b-c frame of reference) as a substitute of regular d-q frame of reference. Unlike the d-q model, the phase variable machine model reflects the rotor position dependence of inductances and flux linkage, low frequency saturation, eddy currents, cogging torque and geometrical effects such as slotting.

The FE model is also considered as a physical model. Though, it is very time consuming computationally. This is surely not desired in machine modeling. Another purpose of developing the phase variable model was to improve the speed of process. 
FE model takes a long time to attain a solution while phase variable model reaches steady state within a very short time compared to FE model. It is as fast as d-q model.

System simulators such as Simulink-MATLAB provide a user-friendly interface for the dynamic modeling of electrical machines. In Simulink, there are various types of power electronic blocks, ready signal processing methods, in addition to the variety of powerful mathematical tools which can be used to study an integrated motor drive system.

Circuit method of implementing the physical phase variable model in Simulink is described below. Equations (3-158) through (3-160) describe the system of electrical equations governing the phase variable model of the machine in the a-b-c frame of reference.

$$
\begin{aligned}
& V_{a b c}=R_{a b c} \cdot i_{a b c}+\frac{d \lambda\left(i_{a b c}, \theta_{r}\right)}{d t} \\
& \lambda\left(i_{a b c}, \theta_{r}\right)=\lambda_{s}\left(i_{a b c}, \theta_{r}\right)+\lambda_{r}\left(\theta_{r}\right) \\
& T_{e}=\left(\frac{P}{2}\right) \times\left(i_{a b c}^{T} \cdot \frac{d\left[\lambda_{r}+L_{a b c} \cdot\left(i_{a b c}, \theta_{r}\right)\right]}{d \theta_{r}}\right)+T_{c o g}\left(\theta_{r}\right)
\end{aligned}
$$

where, $V_{a b c}$ is the terminal voltage vector, $\theta_{r}$ is the electrical angular velocity, $i_{a b c}$ is the current vector in a-b-c frame of reference. Here, $\lambda_{r}\left(\theta_{r}\right)$ is the flux linkage of the armature winding resulted from just permanent magnet. Also, $\lambda_{s}\left(i_{a b c}, \theta_{r}\right)$ is the flux linkage of the armature winding resulted from itself, $P$ is the number of the poles and $T_{\operatorname{cog}}(\theta)$ is the cogging torque. The mechanical equations are similar to the equations (3-129) and (3-130). The terms $\lambda\left(i_{a b c}, \theta_{r}\right)$ is defined as: 


$$
\lambda\left(i_{a b c}, \theta_{r}\right)=\left[\begin{array}{c}
\lambda_{a} \\
\lambda_{b} \\
\lambda_{c}
\end{array}\right]=\left[\begin{array}{lll}
L_{a a}\left(i_{a b c}, \theta_{r}\right) & L_{a b}\left(i_{a b c}, \theta_{r}\right) & L_{a c}\left(i_{a b c}, \theta_{r}\right) \\
L_{a b}\left(i_{a b c}, \theta_{r}\right) & L_{b b}\left(i_{a b c}, \theta_{r}\right) & L_{b c}\left(i_{a b c}, \theta_{r}\right) \\
L_{a c}\left(i_{a b c}, \theta_{r}\right) & L_{b c}\left(i_{a b c}, \theta_{r}\right) & L_{c c}\left(i_{a b c}, \theta_{r}\right)
\end{array}\right] \cdot\left[\begin{array}{c}
i_{a} \\
i_{b} \\
i_{c}
\end{array}\right]+\left[\begin{array}{c}
\lambda_{r a}\left(\theta_{r}\right) \\
\lambda_{r b}\left(\theta_{r}\right) \\
\lambda_{r c}\left(\theta_{r}\right)
\end{array}\right]
$$

\subsection{Phase variable model based line-line voltage modeling for healthy PMSM without access to neutral point $[141-148]$}

The line-line modeling of equations (3-158) to (3-160), enables us to reduce the volume of the model and also facilitate the coupling of the phase variable model with external circuits. The expanded form of the equation (3-158) is:

$$
\begin{aligned}
& {\left[\begin{array}{l}
V_{a} \\
V_{b} \\
V_{c}
\end{array}\right]=\left[\begin{array}{ccc}
r_{a} & 0 & 0 \\
0 & r_{b} & 0 \\
0 & 0 & r_{c}
\end{array}\right] \cdot\left[\begin{array}{c}
i_{a} \\
i_{b} \\
i_{c}
\end{array}\right]+\frac{d}{d t}\left[\begin{array}{c}
\lambda_{a} \\
\lambda_{b} \\
\lambda_{c}
\end{array}\right]} \\
& V_{a b}=V_{a}-V_{b}
\end{aligned}
$$

Assuming:

$$
\begin{aligned}
& V_{a b}=V_{a}-V_{b} \\
& V_{b c}=V_{b}-V_{c} \\
& i_{c}=-i_{a}-i_{b}
\end{aligned}
$$

166)

By subtraction of the first and second row of the equation (3-162) we have:

$$
V_{a b}=r_{a} \cdot i_{a}+\frac{d \lambda_{a}}{d t}-r_{b} \cdot i_{b}-\frac{d \lambda_{b}}{d t}
$$

By subtraction of the second and third row of the equation (3-162) and assuming (3-165), we have:

$$
V_{b c}=r_{b} \cdot i_{b}+\frac{d \lambda_{b}}{d t}-r_{c} \cdot\left(-i_{a}-i_{b}\right)-\frac{d \lambda_{c}}{d t}
$$


The Matrix form of the equations (3-166) and (3-167) is:

$$
\left[\begin{array}{l}
V_{a b} \\
V_{b c}
\end{array}\right]=\left[\begin{array}{cc}
r_{a} & -r_{b} \\
r_{c} & r_{b}+r_{c}
\end{array}\right] \cdot\left[\begin{array}{l}
i_{a} \\
i_{b}
\end{array}\right]+\frac{d}{d t}\left[\begin{array}{ccc}
1 & -1 & 0 \\
0 & 1 & -1
\end{array}\right] \cdot\left[\begin{array}{c}
\lambda_{a} \\
\lambda_{b} \\
\lambda_{c}
\end{array}\right]
$$

The combination of the equation (3-169) with the equation (3-161) and solving it for the current leads to:

$$
\begin{aligned}
& {\left[\begin{array}{l}
i_{a} \\
i_{b}
\end{array}\right]=\int_{0}^{t}\left\{\left[\begin{array}{ll}
L_{a a}-L_{a c}-L_{b a}+L_{b c} & L_{a b}-L_{a c}-L_{b b}+L_{b c} \\
L_{b a}-L_{a c}-L_{b c}+L_{c c} & L_{b b}-L_{b c}+L_{c c}-L_{b c}
\end{array}\right]^{-1} \times\right.} \\
& \left.\left\{\left[\begin{array}{l}
V_{a b} \\
V_{b c}
\end{array}\right]-\left[\begin{array}{cc}
r_{a} & -r_{b} \\
r_{c} & r_{b}+r_{c}
\end{array}\right] \cdot\left[\begin{array}{l}
i_{a} \\
i_{b}
\end{array}\right]-\frac{d}{d t}\left[\begin{array}{ccc}
1 & -1 & 0 \\
0 & 1 & -1
\end{array}\right] \cdot\left[\begin{array}{l}
\lambda_{r a} \\
\lambda_{r b} \\
\lambda_{r c}
\end{array}\right]\right\} \cdot d \tau\right\}
\end{aligned}
$$

\subsection{Phase variable model based line-line voltage modeling for Faulty PMSM without access to neutral point}

Iron saturation, vibration, Magnet demagnetization, electrical resistance change and reluctance changes have been the main interest in physics-based modeling of PMSM machine either by numerical or analytical approach. The Iron saturation, vibration and reluctance change due to eccentricity do not have a real time constant. PM demagnetization and winding resistance have a temperature-dependent time constant which is dependent to the mass, geometry of machine and material properties. The magnets coercivity can be significantly reduced with a temperature elevation of a few tens of degrees Celcius. Over temperature of winding can also burns the winding insulations in a few second. The over temperature of PM happens several seconds after the windings completely heated up due to inter-turns fault. Provided the reaction time of diagnosis system is much lower than the thermal time constant of the windings, therefore, the thermal modeling of the PM concerning fault is not in the first degree of importance. In addition, in almost all of wisely designed PMSM without field weakening windings, the reverse field cannot drop the PM 
operation point below the knee point. However, reversible or irreversible demagnetization modeling due to the reverse field or long term over-temperature helps to enhance the reliability and also reduce the cost of PMSM in design stage.

Computer simulations of inter-turn fault can be accomplished either using finiteelement (FE) models or analytical models. Analytical methods, although broadly used, are unable to precisely model a machine. Generally, the usage of FE method in modeling of electrical machines for fault diagnosis purposes can be classified into four major categories i.e. full FE models, d-q models, the FE-based phase variable model and the field reconstruction method (FRM). The full FE model in a coupled field circuit analysis offer the most accurate modeling methods. A simple d-q model comparing to the others shows great flexibility but it is not useful enough for fault diagnosis purposes under a phase unbalancing condition. FRM is used to estimate the flux linking each stator slot, which is then compared with real measured values to arrive at a fault decision. This method is computationally less intensive comparing to full FE model because only one stator slot is performed in just FE domain and the fields in the other stator slots are reconstructed using this information. Comparing to full FE model and FRM, FE-based phase variable model is less time consuming and more flexible where it can easily be recreated in integrated circuits for variable speed applications. Comparing to a simple d-q model it shows similar simulation run time[83].The FE-based phase variable model of PM machines as a database representation of the transient operation of the electrical machine[141]. The FE-based phase variable model is equipped with additional elements considering the effects of core loss and saturation. In this work, the winding temperature is included to this model. The creation of this model consists of two general steps. In the first step, a non-linear transient FE analysis is done, to calculate the cogging torque, EMF, flux 
linkage and the inductance Matrix of the machine. In the next step these calculated parameters are retrieved in 1D look up Tables inside circuit simulators and the machine voltage and motion equations are simulated employing these look up Tables. For more details refer to [146]. In the FE domain the effects of core saturation, the slotting and also the location and the number of shorted turns are taken into account.

The Inter-turn faults increase the slot leakage flux and cause iron saturation. Under inter-turn fault, the inductances are no longer functions of the number of turns and slot geometry only but are also dependent on the material property and the location of the faulted turns in the slot. Here, for initial fault studies a FE-based model is used. The creation of this model consists of two general steps. In the first step, a non-linear transient FE analysis is performed to calculate the cogging torque, EMF, PM flux linkage and the inductance matrix of the machine. In the next step these calculated parameters are collected in look up Tables to be employed in the machine equation (3-170). The inductances are calculated as a function of rotor position, $\theta$ and the saturation level $\left(i_{d}, i_{q}\right)$. During the inductance calculation from FE analysis, the resultant flux from positive $i_{d}$ and the fault current, $i_{f}$, are set to be exactly in opposite with PM flux linkage. The resultant flux from $i_{q}$ is set to be perpendicular with the PM flux linkage. The line-line model of a Faulty 3-phase PMSM without access to the neutral point under ISCF is expressed as:

$$
\begin{aligned}
& {\left[\begin{array}{l}
i_{a} \\
i_{b} \\
i_{f}
\end{array}\right]=A^{-1}\left\{\int_{0}^{t}\left[\begin{array}{l}
V_{a b} \\
V_{b c} \\
0
\end{array}\right]-B \cdot\left[\begin{array}{l}
i_{a} \\
i_{b} \\
i_{f}
\end{array}\right]-\frac{d}{d t}\left[\begin{array}{cccc}
1 & -1 & 0 & 0 \\
0 & 1 & -1 & 0 \\
0 & 0 & 0 & 1
\end{array}\right] \cdot\left[\begin{array}{l}
\lambda_{r a} \\
\lambda_{r b} \\
\lambda_{r c} \\
\lambda_{r f}
\end{array}\right]\right\}} \\
& i_{a}+i_{b}+i_{c}=0
\end{aligned}
$$




$$
\begin{aligned}
& {\left[\begin{array}{c}
E_{a a^{\prime}} \\
E_{b b^{\prime}} \\
E_{c c^{\prime}} \\
E_{f f^{\prime}}
\end{array}\right]=\frac{d}{d t}\left[\begin{array}{c}
\lambda_{r a} \\
\lambda_{r b} \\
\lambda_{r c} \\
\lambda_{r f}
\end{array}\right]} \\
& T_{e}=\left(\frac{P}{2}\right) \times\left(i_{a b c f}^{T} \cdot \frac{d\left[\lambda r_{a b c f}(\theta)+L_{a b c f} \cdot\left(i_{a b c f}, \theta_{r}\right)\right]}{d \theta_{r}}\right)+T_{c o g}\left(\theta_{r}\right)
\end{aligned}
$$

where the $\lambda r_{a b c f}, A, B$ and $i_{a b c f}$ are defined as:

$$
\begin{aligned}
& \lambda r_{a b c f}(\theta)=\left[\begin{array}{c}
\lambda_{r a}(\theta) \\
\lambda_{r b}(\theta) \\
\lambda_{r c}(\theta) \\
\lambda_{r f}(\theta)
\end{array}\right] \\
& {\left[\begin{array}{c}
E_{a a^{\prime}}(\theta) \\
E_{b b^{\prime}}(\theta) \\
E_{c c^{\prime}}(\theta) \\
E_{f f^{\prime}}(\theta)
\end{array}\right]=\frac{d}{d t}\left[\begin{array}{l}
\lambda_{r a}(\theta) \\
\lambda_{r b}(\theta) \\
\lambda_{r c}(\theta) \\
\lambda_{r f}(\theta)
\end{array}\right]} \\
& i_{a b c f}=\left[\begin{array}{l}
i_{a} \\
i_{b} \\
i_{c} \\
i_{f}
\end{array}\right] \\
& L_{a b c f}\left(\theta, i_{a b c f}\right)=\left[\begin{array}{llll}
L_{a a}\left(\theta, i_{a b c f}\right) & L_{a b}\left(\theta, i_{a b c f}\right) & L_{a c}\left(\theta, i_{a b c f}\right) & L_{a f}\left(\theta, i_{a b c f}\right) \\
L_{a b}\left(\theta, i_{a b c f}\right) & L_{b b}\left(\theta, i_{a b c f}\right) & L_{b c}\left(\theta, i_{a b c f}\right) & L_{b f}\left(\theta, i_{a b c f}\right) \\
L_{a c}\left(\theta, i_{a b c f}\right) & L_{a b}\left(\theta, i_{a b c f}\right) & L_{c c}\left(\theta, i_{a b c f}\right) & L_{c f}\left(\theta, i_{a b c f}\right) \\
L_{a f}\left(\theta, i_{a b c f}\right) & L_{a c}\left(\theta, i_{a b c f}\right) & L_{a b}\left(\theta, i_{a b c f}\right) & L_{f f}\left(\theta, i_{a b c f}\right)
\end{array}\right] \\
& A\left(\theta, i_{a b c f}\right)=\left[\begin{array}{ccc}
L_{a a}-L_{a c}-L_{b a}+L_{b c} & L_{a b}-L_{a c}-L_{b b}+L_{b c} & L_{a f}-L_{b f} \\
L_{b a}-L_{a c}-L_{b c}+L_{c c} & L_{b b}-L_{b c}+L_{c c}-L_{b c} & L_{b f}-L_{c f} \\
L_{a f}-L_{b f} & L_{b f}-L_{c f} & L_{f f}
\end{array}\right] \\
& B=\left[\begin{array}{ccc}
R_{a} & -R_{b} & 0 \\
R_{c} & R_{b}+R_{c} & 0 \\
0 & -R_{f s c} & R_{f}+R_{f s c}
\end{array}\right]
\end{aligned}
$$




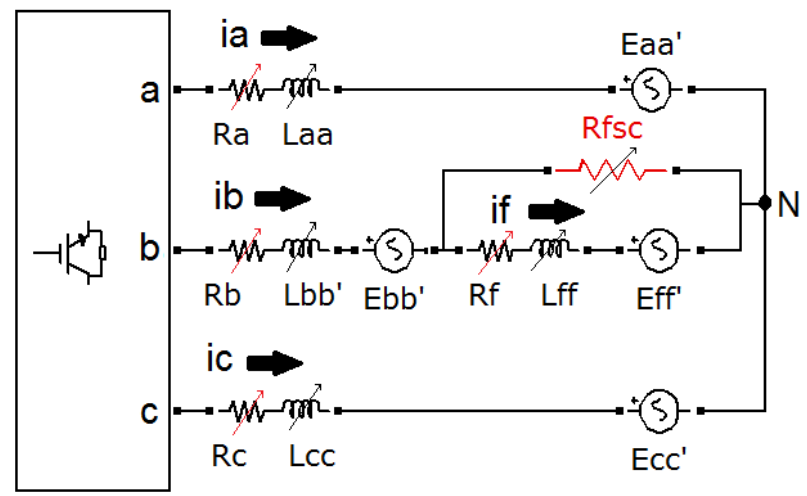

Figure 3.14 Inter-turn for conventional PMSM drives supplied by a 3-phase inverter

\subsection{Phase variable model based line-line voltage modeling for faulty PMSM with access to neutral point}

Typically a 3-phase fault-tolerant PMSM have a sectional structure with concentrated windings without overlapping in order to have electrical and magnetic isolation between phases [148]. In fault-tolerant machines it is preferred to supply the phase separately. For this reason each phase is supplied separately by a H-bridge converter. The schematic view of this configuration is viewed in figure 3.15 . The circuit modeling of such system can be described as:

$$
\left[\begin{array}{c}
i_{a} \\
i_{b} \\
i_{f} \\
i_{c}
\end{array}\right]=A^{-1}\left\{\int_{0}^{t}\left[\begin{array}{l}
V_{a a^{\prime}} \\
V_{b b^{\prime}} \\
V_{f f^{\prime}} \\
V_{c c^{\prime}}
\end{array}\right]-B \cdot\left[\begin{array}{c}
i_{a} \\
i_{b} \\
i_{f} \\
i_{c}
\end{array}\right]-\frac{d}{d t}\left[\begin{array}{cccc}
1 & 0 & 0 & 0 \\
0 & 1 & 0 & 0 \\
0 & 0 & 1 & 0 \\
0 & 0 & 0 & 1
\end{array}\right] \cdot\left[\begin{array}{c}
\lambda_{r a} \\
\lambda_{r b} \\
\lambda_{r f} \\
\lambda_{r c}
\end{array}\right]\right\}
$$

where $f$ is the index of shorted turns; $R_{f s c}$ is the fault resistance on the shorted turns. Here, $E_{f f^{\prime}}$ are the coils EMF, respectively.

The corresponding resistance and inductance matrices are defined by: 


$$
L_{a b c f}\left(\theta, i_{a b c f}\right)=\left[\begin{array}{llll}
L_{a a}\left(\theta, i_{a b c f}\right) & L_{a b}\left(\theta, i_{a b c f}\right) & L_{a f}\left(\theta, i_{a b c f}\right) & L_{a c}\left(\theta, i_{a b c f}\right) \\
L_{a b}\left(\theta, i_{a b c f}\right) & L_{b b}\left(\theta, i_{a b c f}\right) & L_{b f}\left(\theta, i_{a b c f}\right) & L_{b c}\left(\theta, i_{a b c f}\right) \\
L_{a f}\left(\theta, i_{a b c f}\right) & L_{b f}\left(\theta, i_{a b c f}\right) & L_{f f}\left(\theta, i_{a b c f}\right) & L_{f c}\left(\theta, i_{a b c f}\right) \\
L_{a c}\left(\theta, i_{a b c f}\right) & L_{b c}\left(\theta, i_{a b c f}\right) & L_{f c}\left(\theta, i_{a b c f}\right) & L_{f f}\left(\theta, i_{a b c f}\right)
\end{array}\right]
$$

183)

$$
B=\left[\begin{array}{cccc}
R_{a} & 0 & 0 & 0 \\
0 & R_{a} & 0 & 0 \\
0 & 0 & R_{a} & 0 \\
0 & 0 & 0 & R_{a}
\end{array}\right]
$$

It is mentioned that the matrix $\mathrm{B}$ can be thermal dependent where the resistance of the machine is continuously updated by an embedded thermal network. In the last section of this chapter this is explained in a comprehensive manner.

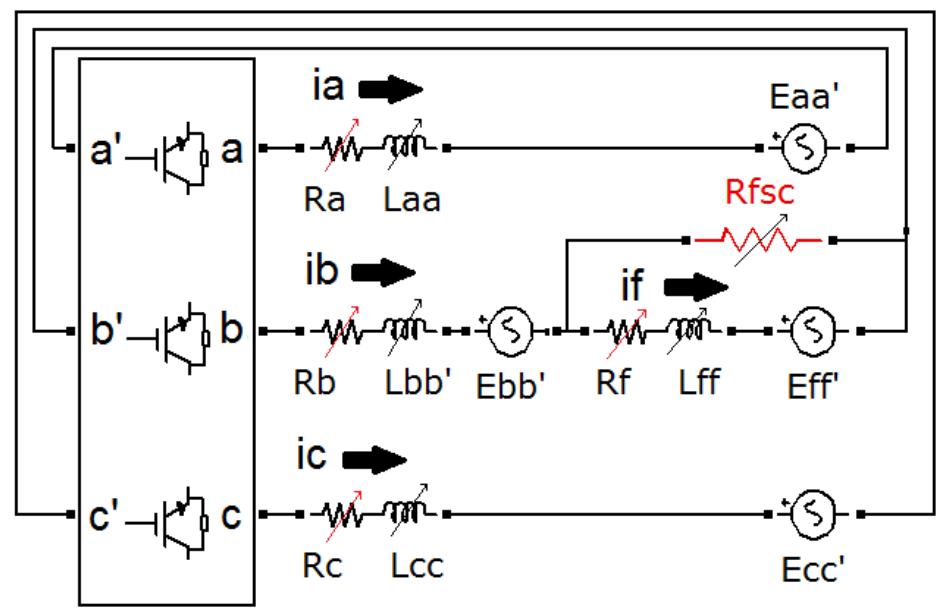

Figure 3.15 Inter-turn fault model for conventional fault tolerant machines supplied by a three single H-bridge inverters

\subsection{Thermo-electric modeling of PMSM taking into accounts the demagnetization issue}

The healthy phase variable satisfies the equation (3-170). The equation (3-170) covers the saturation of iron. However, the thermal aspects and the demagnetization issues are to be added to create the complete physics-based model. In order to create 
the thermo-electric version of the normal phase variable model, the thermal model should embed inside equation (3-170). Doing so, the equivalent resistance matric is not constant anymore, but the elements of this matrix in equations, (3-180) and (3184) change with the temperature as:

$$
R(T)=R_{0}(1+\rho \Delta T)
$$

where $R_{0}$ is the winding temperature at a certain temperature, $\rho$ is the constant coefficient and $\Delta T$ is the difference between instantaneous winding temperature and the certain temperature.

Having the mean temperature of the magnets it is possible to predict the average magnets operating point; however the effect of reverse armature field on magnet should be considered. In order to calculate the effect of reverse armature field on the face of magnet equation (3-121) should be calculated. In order to calculate $\bar{B}_{r r}$, firstly, $\bar{B}_{m r}$ and $\bar{B}_{a r}$ should be calculated. Here, $\bar{B}_{m r}$ can be simply calculated from a non-linear transient FE- analysis with motion neglecting the thermal effects and reverse field as a function of rotor position. A 1-D look up Table is used to collect the $\bar{B}_{m r}$ in Simulink domain. In order to calculate the $\bar{B}_{a r}\left(\theta, i_{d}, i_{q}\right)$ on the PM face, a transfer inductance matrix $\left(\mathrm{T}_{\mathrm{W}-\mathrm{M}}\right)$, equation $(3-186)$, is defined to reflect the winding flux linkage to the PM face by using equation (3-187). In (3-187), $S_{\text {magnet }}$ is the outer face of cubic magnet which is perpendicular to the magnetic flux.

$$
\begin{aligned}
& T_{W-M}=\left\lfloor L_{m a}\left(\theta, i_{d}, i_{q}\right) \quad L_{m b}\left(\theta, i_{d}, i_{q}\right) \quad L_{m c}\left(\theta, i_{d}, i_{q}\right)\right\rfloor \\
& \bar{B}_{a r}\left(\theta, i_{d}, i_{q}\right)=\frac{\left(T_{W-M} \times\left[i_{a}, i_{b}, i_{c}\right]^{T}\right)}{S_{\text {magnet }}}
\end{aligned}
$$

where $L_{m a}, L_{m b}, L_{m c}$ are the mutual inductance between armature and the artificial coil 
located on the side of the magnet. The surface area of artificial coils equals to $S_{\text {magnet }}$. $i_{a}, i_{b}$ and $i_{c}$ are the armature phase currents. $i_{d}$ and $i_{q}$ are the direct and the quadrature current in rotor frame of reference.

Now that the, $\bar{B}_{a r}$ is calculated it is possible to calculate the $\bar{B}_{r r}$ from equation (3121), however, the changes in $\bar{B}_{r r}$ strongly depend on magnet specific temperature data and magnet geometry, therefore it is necessary to modify the $\bar{B}_{r r}$ with respect to the operational temperature. Here, $F_{T B}(T)$ is defined to adapt $\bar{B}_{r r}$ in new temperatures as:

$$
\bar{B}_{r r \text { modified }}\left(T, \theta, i_{d}, i_{q}\right)=\bar{B}_{r r}\left(\theta, i_{d}, i_{q}\right) \times \bar{F}_{T B}(T)
$$

Here, $F_{T B}(T)$ is a quadratic function that modify the $\bar{B}_{r r}$ with respect to PM operational temperature.Also, $F_{T B}(T)$ is a normalized function that is calculated from magnetic data for $\mathrm{H}=0(\mathrm{~A} / \mathrm{m})$. In order, to calculate this function, firstly the values of remnant flux at different temperature for $\mathrm{H}=0(\mathrm{~A} / \mathrm{m})$ is derived from $\mathrm{PM}$ datasheets, then these values are normalized with the remnant flux at $20^{\circ}$ (C) and finally a quadrature function is fitted to these data. $F_{T B}(T)$ may be expressed in the form of :

$$
F_{T B}\left(T_{m a g}\right)=P_{10} \cdot\left(T_{m a g}-20\right)^{2}+P_{20} \cdot\left(T_{m a g}-20\right)+P_{30}
$$

where, $T_{\text {mag }}$ is the mean magnet temperature and for the studied PM, $\left[P_{10}, P_{20}, P_{30}\right]$ are $[-4.5478 \mathrm{e}-6,-0.00079133,1.001]$

For partial demagnetization assessments, after calculation of the average normal $\bar{B}_{r r \text { mod ified }}$, it is needed to calculate the partial normal $\bar{B}_{r r \cdot \bmod i f i e d}$ in the face of the magnet. It is done by multiplying of the average $\bar{B}_{r r \text { mod ified }}$ to another normalized function i.e. $\left|F\left(B_{\min }\right)\right|$. The $\left|F\left(B_{\min }\right)\right|$ enables us to forecast the minimum normal 
magnetic flux density on the face of the magnets from the average magnetic flux density. In order to calculate $\left|F\left(B_{\min }\right)\right|$ several steps are done. Firstly, static 2-D FE analysis with respect to different values of $i_{d}$ is done and the magnetic flux density in the outer face of cubic magnet which is perpendicular to the magnetic flux is sampled. In the next step, the average value of each function corresponding to each $i_{d}$ is calculated. Following this, the minimum value of each function corresponding to each $\mathrm{i}_{\mathrm{d}}$ is derived and it is divided by the average value of each function. The result of this division is called $\left|F\left(B_{\text {min }}\right)\right|$. The $\left|F\left(B_{\text {min }}\right)\right|$ is used to calculate the minimum normal magnetic flux density from the average $\bar{B}_{r r \text { modified }}\left(T, \theta, i_{d}, i_{q}\right)$ as:

$$
\begin{aligned}
& B_{r}\left(T_{\text {mag }}, \theta, i_{d}, i_{q}\right)=\bar{B}_{r r \text { modified }}\left(T_{\text {mag }}, \theta, i_{d}, i_{q}\right) \times\left|F\left(B_{\text {min }}\right)\right| \\
& \left|F\left(B_{\text {min }}\right)\right| \text { may express in the form of: } \\
& \left|F\left(B_{\min }\right)\right|=P_{11} .\left(i_{d}\right)^{2}+P_{21} .\left(i_{d}\right)+P_{31}
\end{aligned}
$$

where $i_{d}$ is the direct current and for the studied magnet $\left[P_{11}, P_{12}, P_{13}\right]$ is $[-0.00017585$, $-0.00042233,0.98577]$.

As it was explained in before the demagnetization is happed when the normal magnetic flux density on magnets drops below the knee point. In order to calculate the risk of demagnetization, in the first step, it is needed to evaluate the operational knee point with respect to temperature changes. Here, the knee point of the PM at different temperatures is derived from PM data sheet and then a quadratic function is fitted to this data in a form of:

$$
B_{\text {knee }}\left(T_{\text {mag }}\right)=P_{12} \cdot\left(T_{\text {mag }}\right)^{2}+P_{22} \cdot\left(T_{\text {mag }}\right)+P_{32}
$$

where $T$ is the magnet temperature and for the studied magnet $\left[\mathrm{P}_{1}, \mathrm{P}_{2}, \mathrm{P}_{3}\right]$ are [1.8409e-6, - 0.0057953, 0.012371]. 
After calculation of knee point using equation (3-192), at the operational PM temperature, $\mathrm{T}$, the distance between the $B_{r}\left(T_{\text {mag }}, \theta, i_{d}, i_{q}\right)$ and the $B_{\text {knee }}\left(T_{\text {mag }}\right)$ is defined as the demagnetization risk function, $D_{\text {risk }}\left(T_{m a g}, \theta, i_{d}, i_{q}\right)$, as:

$$
D_{\text {risk }}\left(T_{\text {mag }}, \theta, i_{d}, i_{q}\right)=B_{\text {knee }}\left(T_{\text {mag }}\right)-B_{r}\left(T_{\text {mag }}, \theta, i_{d}, i_{q}\right)
$$

The $D_{\text {risk }}\left(T_{\text {mag }}, \theta, i_{d}, i_{q}\right)$ can be directly or indirectly after some modification be used to ensure a reliable and optimal flux weakening control at different ambient temperatures at which the PMSM is operating.

Now that the $D_{\text {risk }}$ is determined, it is possible to calculate the equation (3-193). Indeed, the equation (3-193) could predict if the power density of the magnet is reduced due to the irreversible demagnetization. If demagnetization happens the demagnetization factor $K_{d}$ as the relationship between the old and new flux density on the face of the magnet can be expressed in form of:

$$
K_{d}=\frac{\bar{B}_{m r}(\theta) \cdot F_{T B}(T)+\left[-1<\min \left(D_{r i s k}\right)\right] \mid \min \left(D_{r i s k}<0\right)}{\bar{B}_{m r}(\theta) \times F_{T B}(T=20)}
$$

The equation (3-194) is a simple illustration of the magnet remnanet flux density variations due to demagnetization. Evidently, the, flux linkage of the winding as defined in chapter 2 is proportional to this value. Therefore, the model described in equation (3-170) is simply modified concerning demagnetization issue. The modified line-line modeling of a 3-phase PMSM without access to the neutral point may be expressed as:

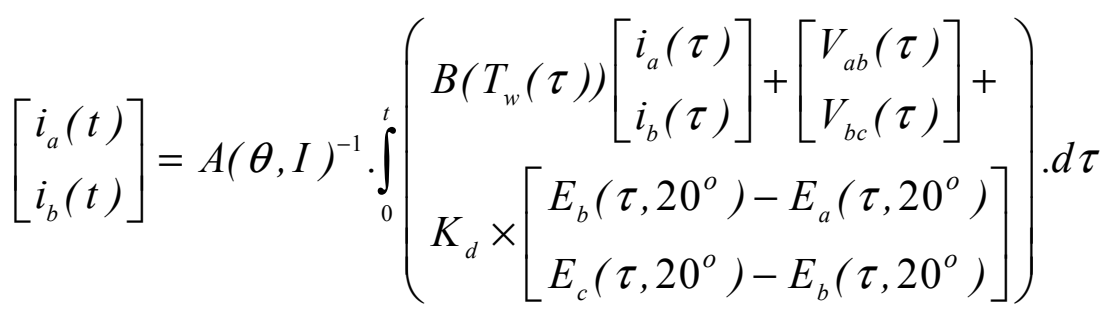


Similarly the torque equation can be modified as:

$$
\begin{array}{r}
T_{m}=\left[0.5 p i_{a b c}^{T} \frac{d L_{a b c}(\theta)}{d \theta} i_{a b c}+i_{a b c}^{T} \frac{K_{d} \times d \lambda_{\text {Rabc }}\left(\theta, 20^{\circ}\right)}{d \theta}\right] \\
+K_{d} \times T_{c o g}\left(\theta, 20^{\circ}\right)[N . m]
\end{array}
$$

where "a", "b" and "c" are phase indices. Also $\mathrm{V}_{\mathrm{ab}}$ and $\mathrm{V}_{\mathrm{bc}}$. are the line-to-line terminal voltage of the machine and $\mathrm{E}_{\mathrm{a}}, \mathrm{E}_{\mathrm{b}}$ and $\mathrm{E}_{\mathrm{c}}$ are the phase-neutral EMF which is equal to $d \lambda_{r}\left(\theta, 20^{\circ}\right) / d \theta . d \lambda_{r}\left(\theta, 20^{\circ}\right)$ is the magnet flux linkages for which the magnet temperature is uniformly set to $20^{\circ} . i_{a}$ and $i_{b}$ are the phase currents, $\mathrm{T}_{\mathrm{w}}$ is the temperature of the armature windings, tis the integration operator, $\theta_{m}=2 \theta / P$ and $\omega_{\mathrm{m}}$ are the mechanical rotor position and speed, respectively. Here $T_{\operatorname{cog}}$ is the cogging torque for which the magnet temperature is uniformly set to $20^{\circ}$ and "I" is $\left(i_{d}, i_{q}\right)$. The matrix $A(\theta, I)$ is the equivalent inductance matrix and $B(T)$ is the equivalent resistance matrix, $K_{d}$ is defined as the demagnetization factor. These parameters are defined as:

$$
\begin{aligned}
A & =\left[\begin{array}{cc}
L_{a a}-L_{a b}-L_{a c}+L_{b c} & , L_{b a}-L_{a c}-L_{b b}+L_{b c} \\
L_{a b}-L_{c a}-L_{b c}+L_{c c} & , L_{b b}-L_{b c}-L_{c b}+L_{c c}
\end{array}\right] \\
B & =\left[\begin{array}{cc}
-R_{a} & R_{b} \\
-R_{c} & -R_{b}-R_{c}
\end{array}\right]
\end{aligned}
$$




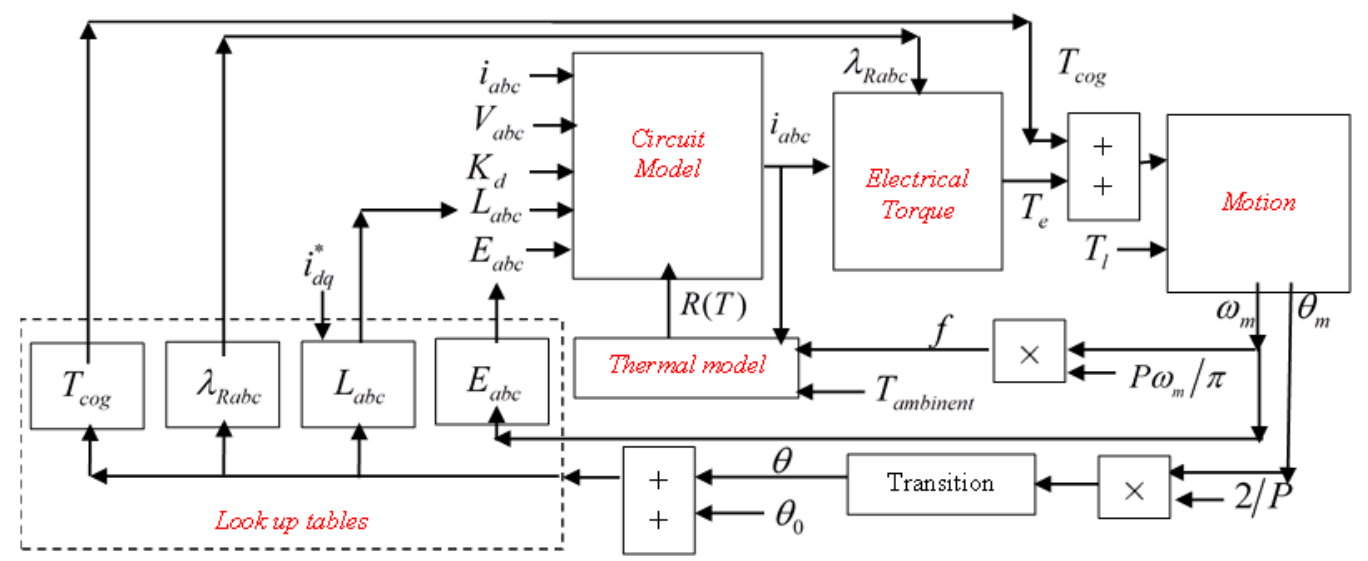

Figure 3.16 Thermo-electric modeling of PMSM taking into accounts the demagnetization issue

\subsection{Summary}

In this chapter, the basic models required for the development of the physicsbased optimization tool, the observers and the estimators, was developed. In the designed models the physical aspects such as the effect of the machine geometry, the core material, the magnet materials and the thermal aspects of the machine were considered in detail. In addition, the d-q mode and the time step FE models were presented for the development of the speed control and the verification systems. The calculation of the machine parameter as the function of the armature current, ambient temperature and rotor position were explained. 


\section{Chapter 4 Control of Permanent Magnet Synchronous Machines}

\subsection{Introduction to the PMSM-drive system}

Interest in Multi-phase PMSM-drive systems[83] has significantly increased in recent decades. The key applications of multi-phase PMSM-drive systems are in electric vehicles, electric traction, hybrid-electric vehicles and the appliance industry. Multi-phase PMSM-drive systems have several advantages. These include high reliability, power density and low torque ripples at higher frequencies.

The PMSM-drive system is defined as a permanent magnet synchronous machine (PMSM) used in the framework of a drive system with rotor position feedback. There are varieties of PMSM-drive system. Generally, these drives may be described by the block diagram in figure 4.1. It consists of four major parts: a power converter, a PMSM, sensors, a control algorithm. The power converter transforms power from electrical source to proper form and gives it to the PMSM which, in turn, converts electrical energy to mechanical energy. One of the noticeable features of the PMSMdrive is the rotor position sensor (or at least the observer). Based upon the rotor position and the command signal, which maybe the torque or voltage or speed command, the control algorithm determines the gate signal to each semiconductor in the power electronic converter.

The configuration of the control algorithm determines the class of drive system. There are three classes; voltage source inverter drives (VSI), current source inverter drives (CSI) and CSI-based-VSI. Both VSI and CSI may be used with PMSM with either sinusoidal or non-sinusoidal EMF waveform. PMSM with a sinusoidal EMF might be controlled somehow to achieve nearly constant torque. The usage of PMSM with a sinusoidal EMF is preferable in applications including flux-weakening and 
high speeds operations. The PMSM with non-sinusoidal back-EMF offers reduced inverter size and reduced losses and higher power density therefore they are preferred where weight or space is a constraint. The usage of cheap Hall rotor position sensors is another benefit that a PMSM with non-sinusoidal EMF offers.

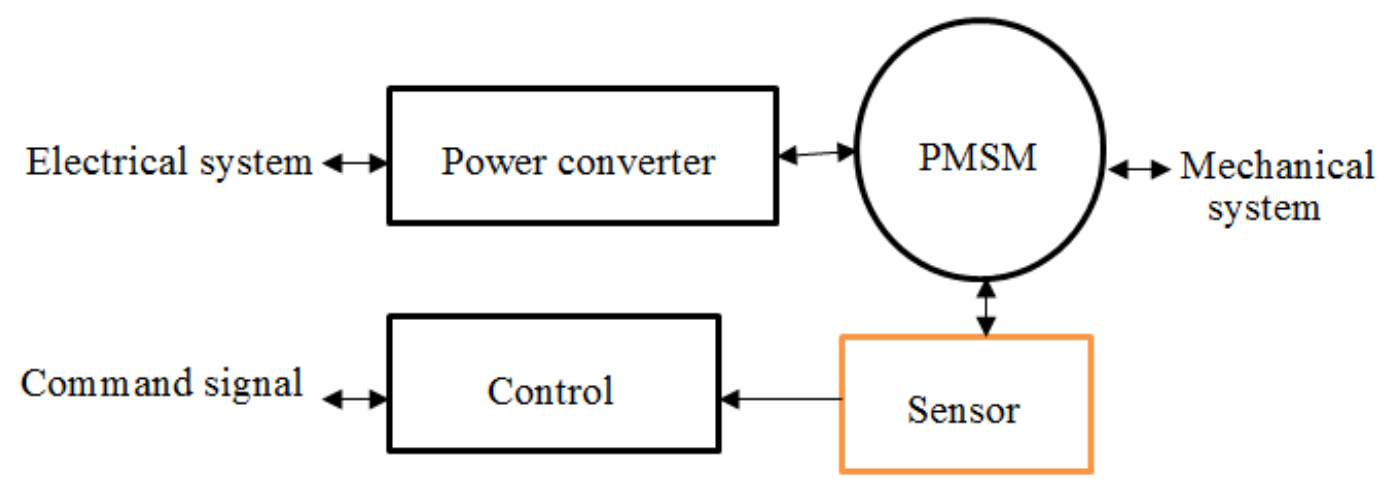

Figure 4.1 PMSM-drive power train

\subsection{Voltage source inverter (VSI) drive for control of PMSM[83]}

In general, in the 3-phase voltage source inverters (VSI) power is supplied from the utility through a transformer. The transformer output is then rectified using a Diode or Thyristor bridges. The rectified output is connected to the de link filter, which may be simply an LC filter. It may also include a stabilizing RLC filter. The filtered rectifier output is used as dc voltage source for the six-switch inverter, which drives the 3-phase PMSM. As shown in figure 4.2, the rotor position, the measured speed and the reference speed are the input to the controller. Based on these inputs, the controller determines the switching states of each of the inverter semiconductor and/or the Thyristors in the DC block. The command signal to the converter and DC block may be quite varied depending upon the structure of the controls in the system in which the drive is embedded. It will often be torque the command; other inputs to the control algorithms may include the dc link voltage, rectifier voltage in DC block. 


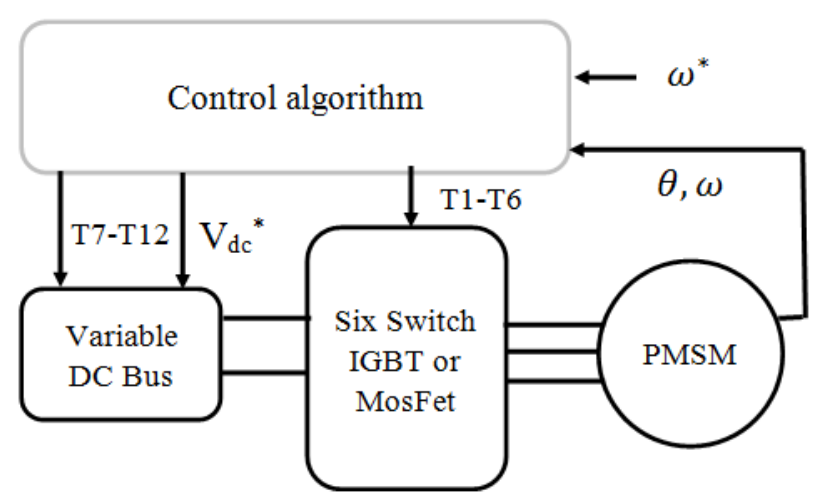

Figure 4.2 Voltage source inverter (VSI) drive for control of PMSM

\subsection{Current-source inverter (CSI) drive for control of PMSM[83]}

Technically, CSI have several discriminative features over VSI. Firstly, the torque is controlled with the same band width by which the stator currents are controlled. In fact, it is often the case that for the practical purposes the torque control is instantaneous. Secondly, is system robustness with regard to changes in machine parameters is high. For instance, CSI are insensitive to parameter variations in the stator leakage inductance or stator resistance. Current regulated drives are also more stable during machine internal faults. For instance, in the event of turn-to-turn short circuit fault within the machine, the currents are automatically limited, which prevents damage to the drive. The phase currents are also automatically limited during start up.

In CSI the speed error is sent to a PI controller. The output is the torque command. A current command synchronizer is then used to determine the q-axis current required to obtain the desired torque with subject to command q-axis current limitation. In CSI controller, the command d-axis current is determined by flux weakening control generally when the phase-neutral EMF reaches the phase-neutral terminal voltages. Based upon the difference between the commanded and actual current, the current regulation, which is normally a Hysteresis or Delta modulation, determines the states 
of the switches T1 to T6.

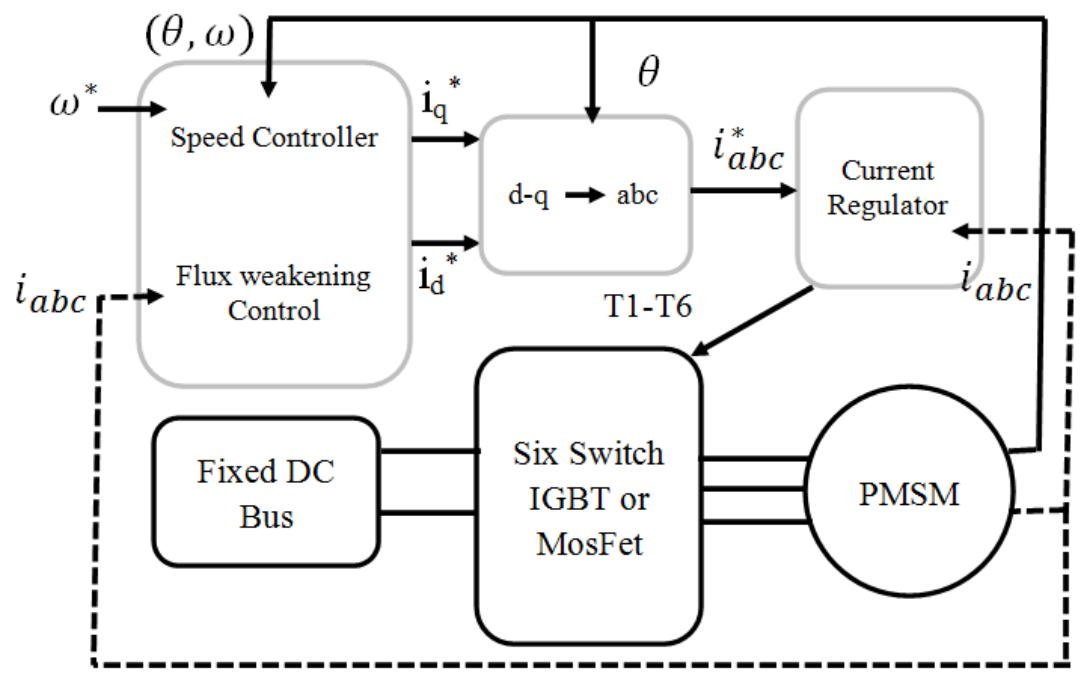

Figure 4.3 Current regulated drive

\subsection{Conventional CSI-based-VSI drive systems[83]}

Another technique to implement a CSI drive is to employ a current control loop on a VSI inverter drive. This is called CSI-based-VSI which is illustrated in figure 4.4. Based upon the commanded q- and d-axis current and the measured q-and d-axis current, the $\mathrm{q}$ and $\mathrm{d}$ - axis reference voltage command are determined. The inverse $\mathrm{d}-\mathrm{q}$ transform is used to calculate the variable voltage command in the a-b-c frame. This is then scaled to calculate the instantaneous duty cycles, or the sine-triangle modulation. As mentioned earlier, the commanded q- and d-axis voltage are in the rotor frame of reference and therefore it needs to be transformed into a stationary frame if the usage of space vector modulation is preferred.

It is beneficial to compare the CSI and CSI-based methods. There are two main differences between the CSI and CSI-based-VSI drives. Firstly, in the CSI-based VSI drives, the terminal voltage is calculated inside controller while in the CSI drive there is a need to measure the terminal voltage of the machine using shunt resistances or 
potential transformers. Secondly, the control algorithm in the CSI is simpler than the CSI-base voltage source inverter drives. The tendency to CSI-based-VSI has been higher in modern drives which are mainly due to the elimination of the need for the terminal voltage measurement.

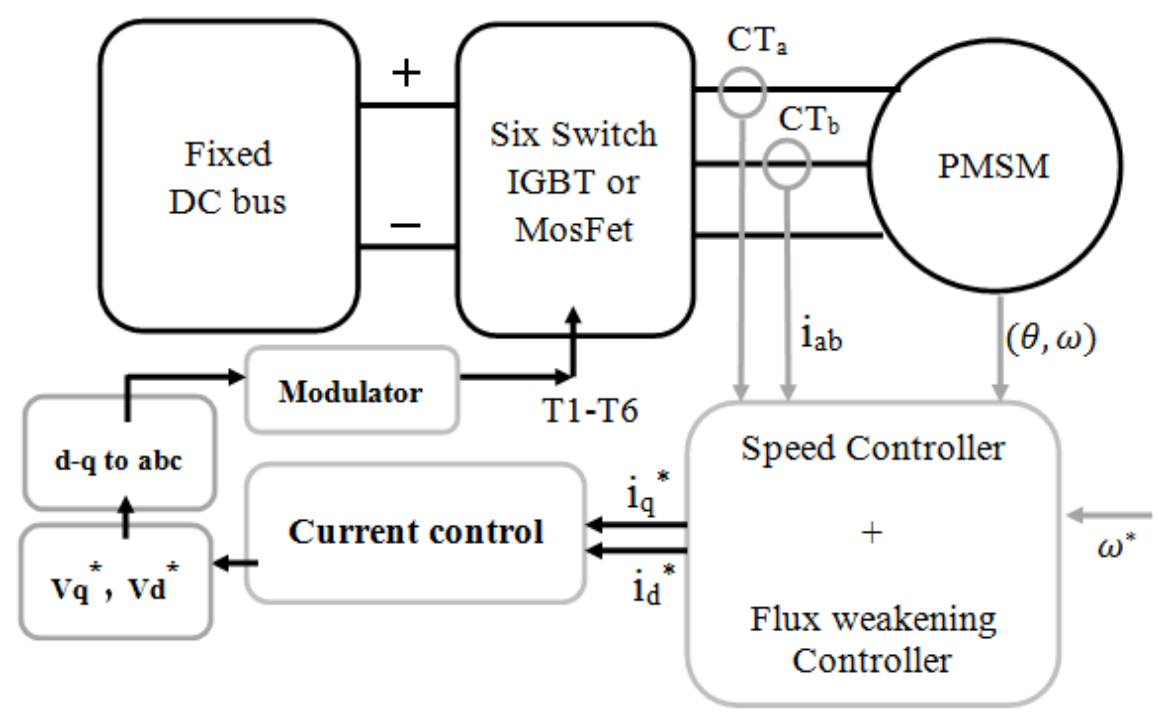

Figure 4.4 Current regulator based voltage source inverter

\subsection{Modern CSI-based-VSI drive systems}

Figure 4.5 illustrates the schematic view of modern PMSM-drive systems with detailed control algorithms that may exist inside it. In general, for speed control, the rotor two parameters are needed i.e. the phase current of the machine and the rotor position. The phase current is measurable by two current transformer or alternatively by shunt resistances. The rotor position can be measured by several methods. It can also be estimated by senseless control method. The speed is calculated by derivation of the incremental rotor position. The speed PI controller is adjusted to determine the command q- axis from the speed error. In addition, in applications requiring the flux weakening operation, this control can be added in parallel with the speed controller to facilitate the speed controllability if DC bus voltage limitation of the drive was met. 
The other role of the flux weakening control is defined when fault diagnosis or prognosis system is trigged by a fault. The fault diagnosis or prognosis system send the message to the flux weakening and to the speed controller in a certain procedure in order to reduce the effect of the fault on the machine. In addition the thermal model of the machine can be embedded in the drive control for fault diagnosis and physicsbased control methods. Because in the model-based sensorless control, fault diagnosis and fault prognostic systems the terminal voltages are needed, the CSI-based VSI drives are preferred to the CSI. In addition, a model-based demagnetization assessment is embedded inside the drive to prevent the irreversible demagnetization during the flux weakening operation and/or high ambient temperature. In this chapter, the demagnetization assessment and control is presented. The model-based rotor position estimation and the model-based fault diagnosis and prognostic system are presented in the later chapters.

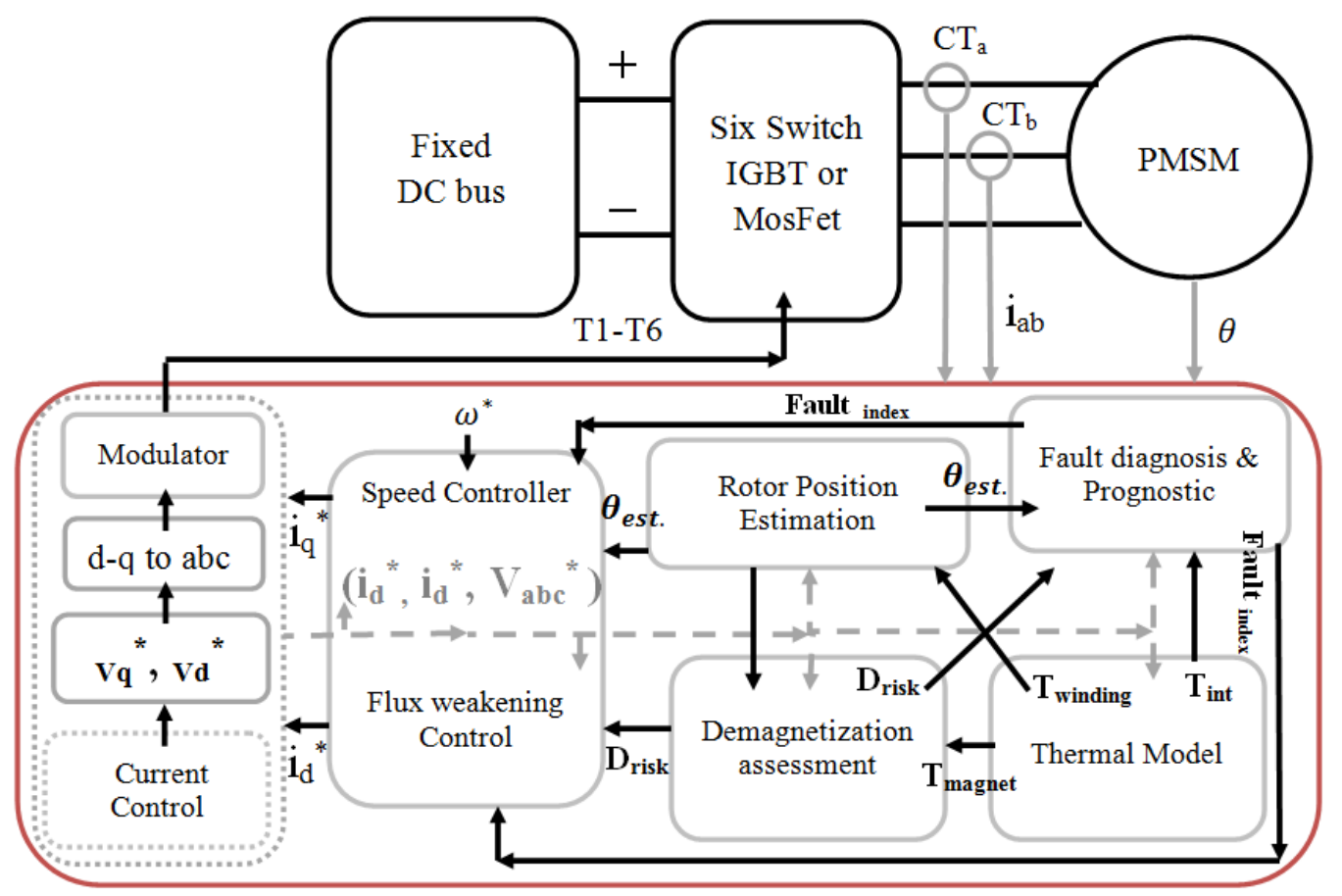

Figure 4.5 Detailed PMSM-VSI drive with current control loop with additional control and diagnosis systems 


\subsection{Modulation techniques}

In order to determine the switching state of the inverter from the commanded current or voltage, the use of the modulation technique is necessary[79,62]. The modulation technique is for a PMSM with fundamental sinusoidal EMF waveform. The traditional modulation techniques in CSI-based-VSI are 180-degree, the duty cycle modulation (PWM) and the space vector modulation[83].

\subsubsection{0-degree modulation technique}

The 180-degree modulation technique is the simplest technique in terms of generating the signal required to control the inverter. It is the based upon the use of the relatively inexpensive Hall Effect position sensors. Therefore this technique is a cheap one. Moreover, because the switching speed of the semiconductors corresponds to the speed of the machine, fast semiconductor switching is not necessary and switching losses will therefore be insignificant. In this modulation technique the inverter switching is six times of the fundamental frequency of applied voltage. However, the inverter does produce considerable harmonic content that will result in increased machine losses. In the 180- degree modulation, the on or off status of the semiconductor is directly tied to electrical rotor position, which is accomplished through the use of the Hall-effect sensors. These sensors can be configured to have a logical high output when they are under a south magnetic pole and a logic zero when they are under a north magnetic pole of the permanent magnet. The drive is extremely easy to implement in hardware; however, at the same time it is difficult to utilize in speed control system, because the fundamental component of the applied voltage cannot be regulated unless a phase controlled rectifier is used. Even though this is certainly possible, it is conventionally preferred to control the applied voltage from the inverter rather than the rectifier because it minimizes the total number of the 
controlled power semiconductor device. In addition, the harmonic content of the phase current, the speed ripples in a PMSM with sinusoidal back EMF becomes high when the drive voltage is modulated by this method. In order to control the amplitude of the fundamental component of the applied voltage or a duty cycle PWM can be used. In this case, the gate drive signals T1-T6 are modulated in order to control the amplitude of the applied voltage. By utilizing the PWM, the amplitude of the applied voltage is readily varied. However, due to the increased switching frequency, the switching loss in the converter is increased. The losses in the machine will be similar to the 180-degree modulation without PWM. This modulation is preferred in VSI drives for PMSM with trapezoidal back EMF[65].

\subsubsection{Sine triangle modulation}

A sine triangle modulation, can be also be used to control the amplitude of the voltage applied to the machine. However, Hall Effect sensors are not generally adequate to sense the rotor position because the phase leg duty cycle is continuous function of the rotor position. For this reason, a resolver or an optical encoder, or observer must be used as a rotor position sensor. Although, it increases the cost of the system and also increases the switching losses of the electronic devices, this technique has does have an advantage in that the low frequency harmonic content of the machine current are generally reduced. Thereby reducing losses in the machine with sinusoidal back EMF are well as reducing the acoustic noise and torque ripple.

\subsubsection{Space vector modulation strategy}

The final voltage source modulation strategy is space vector modulation. This strategy is designed to accept q-and d-axis voltage command in the stationary frame as an input which is obtained by transforming the voltage command in the rotor reference frame and utilizing frame-to-frame transformation and the fast average of 
the output q- and d-axis voltage are equal to the input, provided the peak commanded line-neutral input voltage magnitude is less than a certain value. If this certain limit is exceeded, the q- and d-output voltage vector retains its commanded direction, but the amplitude is limited. The fast average $\mathrm{q}$ - and d-axis voltage of the introduced modulation technique may be expressed as[83]:

$$
\left\{\begin{array}{l}
\bar{V}_{d s}^{r}=-\bar{V}_{d c} \cdot m \cdot \sin \left(\phi_{v}\right) \\
\bar{V}_{q s}^{r}=\bar{V}_{d c} \cdot m \cdot \cos \left(\phi_{v}\right)
\end{array}\right.
$$

where

$$
m= \begin{cases}\frac{2}{\pi} & \text { 180 degree modulation } \\ \frac{2 d}{\pi} & \text { Duty cycle modulation } \\ \frac{d}{2} & \text { Sine triangle modulation } \mathrm{d} \leq 1 \\ \frac{2 f(d)}{\pi} & \text { Sine triangle modulation } \mathrm{d}>1 \text { and } f(d)<1 \\ \frac{d}{2} & \text { Third Harmonic injection } \mathrm{d} \leq 1 \\ \frac{V_{s p k}^{*}}{V_{d c}} & \text { Space vector modulation } V_{s p k}^{*} \leq \frac{V_{d c}}{\sqrt{3}} \\ \frac{1}{\sqrt{3}} & \text { Space vector modulation } V_{s p k}^{*}>\frac{V_{d c}}{\sqrt{3}}\end{cases}
$$

The $\phi_{\mathrm{v}}$ is the angle that is used to set converter voltage or current angle and the rotor position. Beside the values of the commanded q- and d-axis current, the $\phi_{\mathrm{v}}$ has direct influence on the actual applied values of the reference direct and the quadrature current when $d-q$ to $a-b-c$ transformation is used. In surface mounted permanent magnet machines with no saliency, in order to get the maximum torque per Ampere, the reference direct current is set to zero. As long as the $\phi_{\mathrm{v}}$ is properly set to transform this zero current to a-b-c frame of reference, the maximum torque per Ampere is achieved. If not, a direct current is mistakenly applied to the machine which is a 
source of a non-reasonable heat and also the overall flux linkage reduction in the machine. Here, $V_{s p k}^{*}$ is the space vector calculated from the reference direct and the quadrature current in the rotor frame of reference.

\subsection{Flux weakening control}

The pure constant PM Field excitation confines the controllability of PMSM at higher speed than the nominal speed if the DC bus voltage of the drive is fixed and/or limited in a certain boundary. In addition, for higher speeds than nominal speed, PMSM are generally operated in such a way that the armature currents are set with large negative direct-axis component. This is to weaken the flux linkage of the armature windings. This is called flux weakening. This approach might cause to the irreversible demagnetization. To prevent the partial or global irreversible demagnetization of PMs, many solutions were proposed in terms of rotor structures of PMSM. In [1], a new design concept of PM machine for flux-weakening operation has been proposed. The solution was aimed to minimize the amount of required demagnetizing current for a given level of flux weakening. It is done by the altering the flux path of magnets. Although the copper loss reduced but also the risk of damaging the permanent magnets is eliminated, the power density of the machine is decreased. In [149] a magnetic structure termed the consequent-pole PM machine was explored. Due to its particular permanent magnet configuration, this machine allows for a wide range of control of the air-gap flux with minimum field ampere-turn requirements and without brushes or slip rings. This magnetic structure is stated as to have inherent field weakening capability. In [149], several design shapes were reviewed concerning their flux weakening abilities. For instances, changing the stator winding configurations; or by altering the reluctance path of the permanent magnets; 
or by means of a field winding; or by using a two-section rotor PMSM.

In [150], it is stated that for the maximum torque field-weakening control, the optimal design of interior PM motor design concerning high-saliency ratio, gives promising results for applications requiring a wide field-weakening range.

The machine design side approaches were applied on different types of PM motor designs, which may lead to increased manufacturing cost due to the complicated rotor structure or additional field weakening windings.

On the other hand, many control strategies and algorithms have been developed for flux-weakening operation of PMSM [151-158]. In [154], a feed-forward current regulated flux-weakening control algorithms were proposed to improve the torque production capability of the PMSM-drive at high speeds, the efficiency of the this method robustly is associated with the accurate knowledge of the machine parameters where the control performance is degraded significantly as errors between the assumed and actual parameters are enlarged.

In [151], a current-regulated flux-weakening method is developed. In this method, a negative current component is applied on direct-axis to reduce the resulting magnet's flux linkage. This armature reaction effect was used to extend the operating speed range of PMSM and relieve the current regulator from saturation that is subject to occurring at high speeds. The feed forward decoupling compensation were proposed in[155], [156]. In these flux-weakening schemes, the demagnetizing current command was calculated based upon the mathematical model of the PM motor and, consequently, the performance of the PMSM drive system was strongly dependent on the motor parameters and sensitive to the operating conditions. $\operatorname{In}[158]$, a fluxweakening control for surface mounted PMSM, which was relatively simple and did not require the knowledge of the machine and inverter parameters was proposed. In 
addition, the miscellaneous voltage drops such as semiconductors voltage drops, current sensor voltage drops and those caused by the dead-time in the switching strategy were automatically included into the calculation of the direct-axis current. However, the proposed feedback control suffers from control error. Hence, the stability of the overall drive system would be a problem.

In [157], a voltage-based flux-weakening control method was proposed. It is based on a voltage regulator using the voltage error signals between the voltage command and the allowable maximum output voltage. The output of the voltage regulator is set to determine the required amount of the d-axis current.

Both voltage-based and current-based flux-weakening control methods need additional PI regulator, integrator or low pass filter to generate the d-axis current command. This leads to increased complexity in the overall control system. Moreover, the controller could operate appropriately when they are well-tuned. In addition, the magnet's demagnetization prevention under high ambient temperature is not predicted in these controllers.

\subsection{Current control in CSI-based VSI[65]}

Conventionally after determination of command q- and d- current by respective speed control loop and flux weakening control, two current regulators in the rotating reference frame are required to calculate the $\mathrm{q}$ - and d-axis voltage command. Unfortunately, the q- and d-axis current cannot be truly controlled independently due to their cross-coupling effects inside the PMSM. The cross-coupling increases with the increase in rotor speed. As a result, the dynamic performance of current and torque response is degraded at high speeds without decoupling control. This effect is more obvious at PMSM with higher saliency ratio.

As discussed in the chapter 3, the dynamic equation of a PMSM in the rotor 
reference frame can be written in the matrix form as:

$$
\left\{\begin{array}{l}
V_{d s}^{r}=\left(r_{s}+p L_{d}\right) i_{d s}^{r}-\omega_{r} L_{q} i_{q s}^{r} \\
V_{q s}^{r}=\left(r_{s}+p L_{q}\right) i_{q s}^{r}+\omega_{r} L_{d} i_{d s}^{r}+\omega_{r} \lambda_{m}^{\prime r}
\end{array}\right.
$$

where for interior PMSM, saliency, $L_{d} \neq L_{q}$ and for surface mount PMSM $L_{d}=L_{q}$ and in steady state $p L_{d}=0$ and $p L_{q}=0$. The electromagnetic torque is expressed in terms of steady state currents in form of:

$$
T_{e}=\left(\frac{3}{2}\right)\left(\frac{P}{2}\right)\left(\lambda_{m}^{\prime} \cdot i_{q}^{r}+\left(L_{d}-L_{q}\right) i_{q s}^{r} \cdot i_{d s}^{r}\right)
$$

The current regulators are normally a PI controller. In a variable speed-drive, another PI controller is used to control the speed. The conventional control scheme assumes that the torque and the stator flux can be controlled undependably. In the surface mounted PMSM with $L_{d}=L_{q}$, this is simply achievable through controlling the decoupling $\mathrm{q}$ - and $\mathrm{d}$-axis current. This is performed by feed-forward compensation. The feed-forward compensation adds additional term tor the q- and daxis command voltages in order to equalize the control loop to the machine equation. The cross coupling might be cancelled in steady state through the feed-forward voltage command which are determined by the output of the current regulators. Doing this the CSI based VSI control loop is defined as:

$$
\left\{\begin{array}{l}
V_{d s}^{r *}=\left(K_{p}+\frac{K_{i}}{s}\right) \cdot\left(i_{d s}^{r^{*}}-i_{d s}^{r}\right)-\omega_{r} \cdot L_{q} \cdot i_{q s}^{r} \\
V_{q s}^{r^{*}}=\left(K_{p}+\frac{K_{i}}{s}\right) \cdot\left(i_{q s}^{r^{*}}-i_{q s}^{r}\right)+\omega_{r}\left(\lambda_{m}+L_{d} \cdot i_{d s}^{r}\right)
\end{array}\right.
$$

The feed forward compensation leads to a linear relationship between the q- and d-axis currents. There are other methods for decoupling to establish a linear relation between the q-and d-axis current. For example, this is done by bypassing the direct 
role of the q- or d-axis current in the control loop. In [159], it was assumed that by fixing the q-axis command voltage, the command d-axis current can be used as the single control parameter for the whole current regulator. From equation (4-3), it is observed that by fixing the q-axis voltage and neglecting $r_{s}$, a relationship between the d-axis current and the speed is achievable. Therefore, in this method, the speed can be controlled by d-axis current rather than conventional q-axis current. The determination of the proper value for the q-axis command voltage is an issue in this method.

\subsection{Demagnetization control for reliable flux weakening control in PM synchronous machine}

As previously discussed, the flux weakening control in a PMSM-drive system is assisted for two important reasons. Firstly, it helps to reduce the back electromotive force (EMF) at higher speeds than nominal speed. This is very desirable in PMSM especially with saliency; and it is done due to the limitations that exist in increasing the DC bus voltage of the inverter. Secondly, this control might be employed during an inter-turn fault conditions in order to reduce the unwanted circulating current in shorted turns. The flux weakening capability in PMSM designs are different. In some special PMSM designs it can reduce up to $100 \%$ of the PM remnant flux density. This

powerful flux weakening is capable of PM irreversible demagnetization if underestimated.

There are several reasons for PM demagnetization in a PMSM such as low quality of magnet, magnet geometry inside a PMSM, PM thickness, ambient temperature, reverse armature field, radiation and mechanical stress in addition to other factors. The most important traits for PM demagnetization in a PMSM-drive are the reverse 
magnetic loading and over temperature. In PMSM-drive, the reverse magnetic loading on PM is intentionally imposed by flux weakening control and the temperature is unintentionally imposed by Ohmic loss, core loss, PM eddy current loss and the ambient temperature.

Having realistic information about the PM situation by offline or online PM demagnetization assessment, leads to several advantages. In the design stage using offline demagnetization assessment, more reliable and optimal designs can be achieved. This means that, the risk of partial demagnetization can be reduced in the operational environment. Also, the choice of larger machine volume and/or thicker PM, which is a direct consequence of PMSM design for the worse operational case, can be reconsidered. It also leads to wider torque-speed range without sacrificing the other objectives of the design. In operational environments, using online demagnetization assessment, the reliability of the PMSM equipped with flux weakening control at variable ambient temperature and/or emergency conditions is guaranteed. Consequently, the offline or online demagnetization assessments and control are vital key to the realistic, optimal and reliable flux weakening control in PMSM drive with high flux weakening capability. In chapter 3, real-time estimation of the operating point on the face of the PM in PMSM-drive using a physics-based model was presented. The estimated PM operating point is used for reliable flux weakening control which is presented in this chapter. For demagnetization control the following steps are necessary:

- Design of the q-and d-axis current limiter

- Modeling of the magnet

- Calculation of the demagnetization risk

- Calculations of transfer Inductances 
- Calculation of operational PM temperature

- Modeling of PMSM taking into account the demagnetization issue

In chapter 3 , the thermal and the reverse field demagnetization effects were explained. Furthermore, the calculation of the risk of demagnetization using a physics-based model was presented. Also, the calculation of the required parameter for model evaluation and the modification of the normal FE-based phase variable model concerning demagnetization were presented in chapter 2 . In this chapter, a current limiter is designed for demagnetization control. A case of the study is presented and finally the results and conclusions are presented in detail.

\subsubsection{Design of current limiter for reliable flux weakening control}

The flux weakening research area included two domains. The first domain concentrates on the rectification of the PMSM design for reducing the risk of demagnetizing and/or increasing the flux weakening capability of the machine. The second area focuses on control strategies to widen the torque-speed profile and to apply proper the remedial actions during abnormalities. As mentioned earlier, in PMSM CSI-based VSI drives, the commanded q- axis current, $i_{q 0}^{*}$, is an indication of the torque and it is calculated from the speed error using a PI controller. On the other hand, the commanded d-axis current, $i_{d 0}^{*}$, is used for flux weakening control in order to reduce the flux linkage to the armature winding due to PM remnant flux density. As mentioned earlier, decreasing the reference, $i_{d 0}^{*}<0$, takes the operating point toward the knee point in two ways i.e. it heats up the magnet and it produce a direct reverse armature field on magnets. Therefore, in order to avoid dropping the operating point below the knee point and at the same time use the flux weakening control in any ambient temperature, it is needed to properly limit the $i_{d 0}^{*}$. This is performed by 
knowing the fact that how far the magnet operating point is allowed to be from knee point. This means that $D_{\text {risk }}-B_{o} . B_{o}>0$ as a constant security margin. The minimum $B_{\mathrm{o}}$ can be zero. Here, a PI controller is designed to prevent dropping the operating point below, $B_{\text {knee }}+B_{o}$. The output of this PI controller is a positive direct current, $i_{d r}^{*}>0$, see figure 4.6. The PI controller was designed in equation (4-6). The $i_{d r}^{*}$ is the main inputs of a current limiter that bound the reference $i_{d 0}^{*}$ and $i_{q 0}^{*}$, see figure 4.7. Also, $I_{\max }$ is the maximum phase current and $i_{d \max }$ is the maximum allowed direct current, see figure 4.7.

$$
i_{d r}^{*}=K_{p} \cdot\left[\left(D_{r i s k} \leq B_{0}\right) \times D_{r i s k}\right]+\frac{K_{i}}{\tau} \int\left[\left(D_{r i s k} \leq B_{0}\right) \times D_{r i s k}\right] . d t
$$

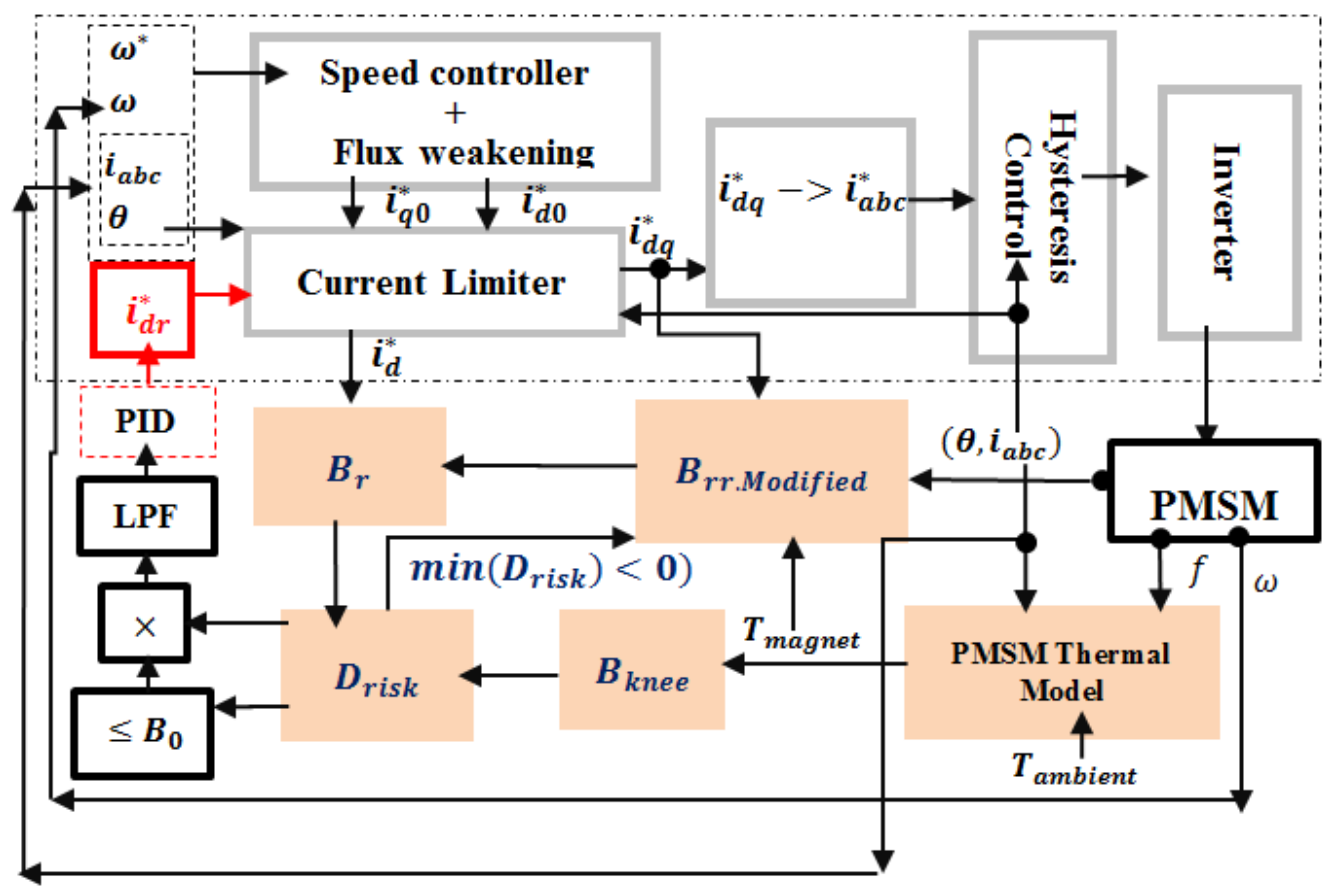

Figure 4.6 Demagnetization control in PMSM based current regulated drive 


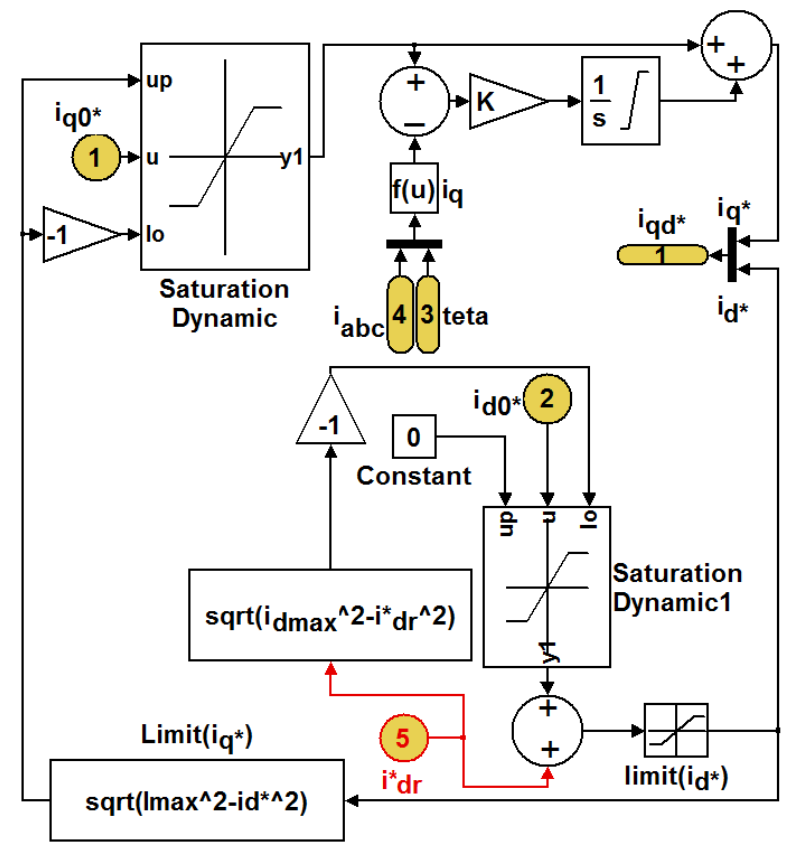

Figure 4.7 Schematic diagram of current limiter

\subsubsection{Case of study}

A 860-W, 6-pole, 85-V V-type PMSM was tested. The PM demagnetization security margin for $\left(T_{\text {magnet }}, i_{d}^{*}\right)=(1200,0)$ and for $\left.T_{\text {magnet }}, i_{d}^{*}\right)=(300,-25)$ is around $0.1-\mathrm{T}$. The maximum phase current, $I_{\max }$, is $27-\mathrm{A}$; the maximum direct current, $I_{\mathrm{dmax}}$ , is $-25-\mathrm{A}$. The maximum speed is $123(\mathrm{rad} / \mathrm{s})$. The rated speed is $70(\mathrm{rad} / \mathrm{s})$, the resistance/phase at $20^{\circ}(C)$ is $0.0476(\Omega)$, the stator core material is M19 and the rotor is steel 1018. The shaft is stainless steel. The complete machine geometry as well as the winding arrangement is illustrated in figures 4.8(a) and 4.8(b) and Table 1, respectively. The machine stack length is $25.4(\mathrm{~mm})$. The current density is 3.5 ( $\left.\mathrm{A} / \mathrm{mm}^{2}\right)$; winding factor is 0.7 ; area of slot is $372\left(\mathrm{~mm}^{2}\right)$; number of conductors per slot is 26 .

The time constant of PM demagnetization, equation (3-121), $\tau_{m}$, is $0.01(\mathrm{~s})$. In 
simulations in order to keep the accuracy using a low sampling time while simulating a relatively large time span, the capacitances of thermal network are reduced to accelerate the machine thermal process. For simulation studies, a hysteresis current regulator was used in a 3-phase IGBT AC drive. Neglecting the control algorithm of flux weakening, the value of $i_{d 0}^{*}$ is selected manually. The value of $i_{q 0}^{*}$ is calculated from the speed error using a PI controller. The load, $T_{L}$, is modeled as $K \times \omega_{m}$ where $K$ is a constant number.

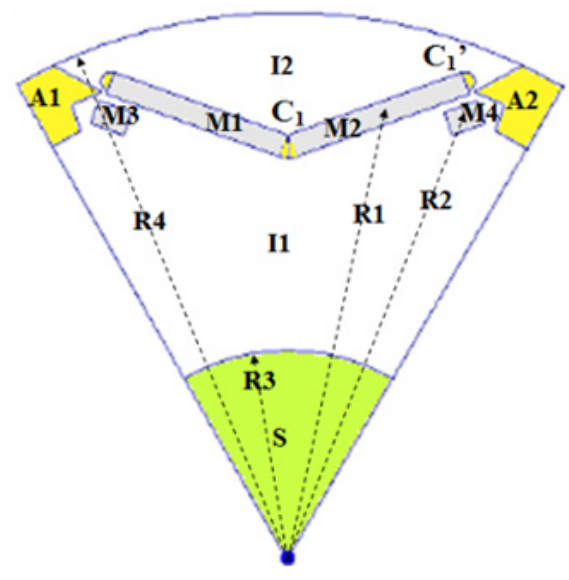

(a)

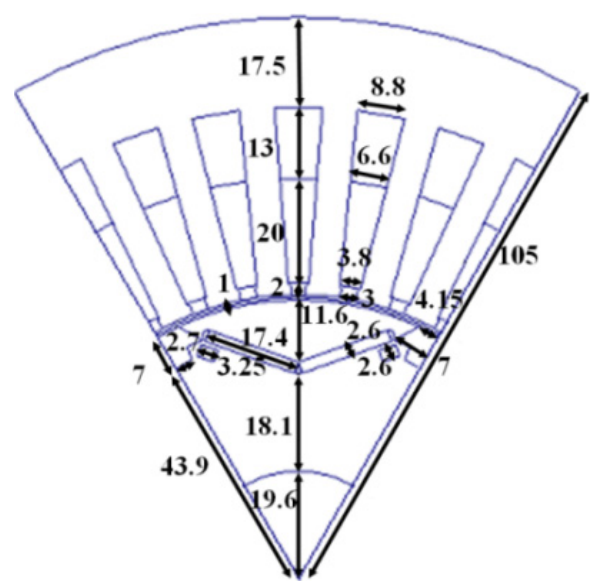

(b)

Figure 4.8 Rotor geometry (a) dimension (b) of V-type PMSM, artificial coil $\mathrm{C} 1-\mathrm{C} 1$ ' is located on flux barrier areas of M2

Table 4.1 Winding arrangement of the studied machine

\begin{tabular}{|c|c|c|c|c|c|c|c|c|c|c|}
\hline $\begin{array}{c}\text { Slot } \\
\text { Number }\end{array}$ & 0 & 1 & 2 & 3 & 4 & 5 & 6 & 7 & 8 & 9 \\
\hline Top & $a+$ & $\mathrm{a}+$ & c- & $c-$ & $\mathrm{b}+$ & $\mathrm{b}+$ & a- & a- & $\mathrm{c}+$ & $\mathrm{C}+$ \\
\hline Bottom & b- & $a+$ & $\mathrm{a}+$ & $\mathrm{a}+$ & c- & c- & $\mathrm{b}+$ & $\mathrm{b}+$ & a- & a- \\
\hline
\end{tabular}

\subsubsection{Model of the magnet}

Figure 4.9 shows a typical demagnetization curves for sintered magnet on a $\mathrm{Nd}$ Fe-B base used in the simulations. In order to have a close form for this data, it is preferred to interpolate this surface in the form of figure 4.10 by using equation (3- 
118). Here, $B_{r}$ is the remnant flux density, $T$ is the temperature and $H$ is normal flux intensity. Also, $\left[P_{00}, P_{10}, P_{01}, P_{11}, P_{22}\right]$ are the constant vector of the interpolated surface as $[-1218.4,7.5,148.6,-4,535]$.

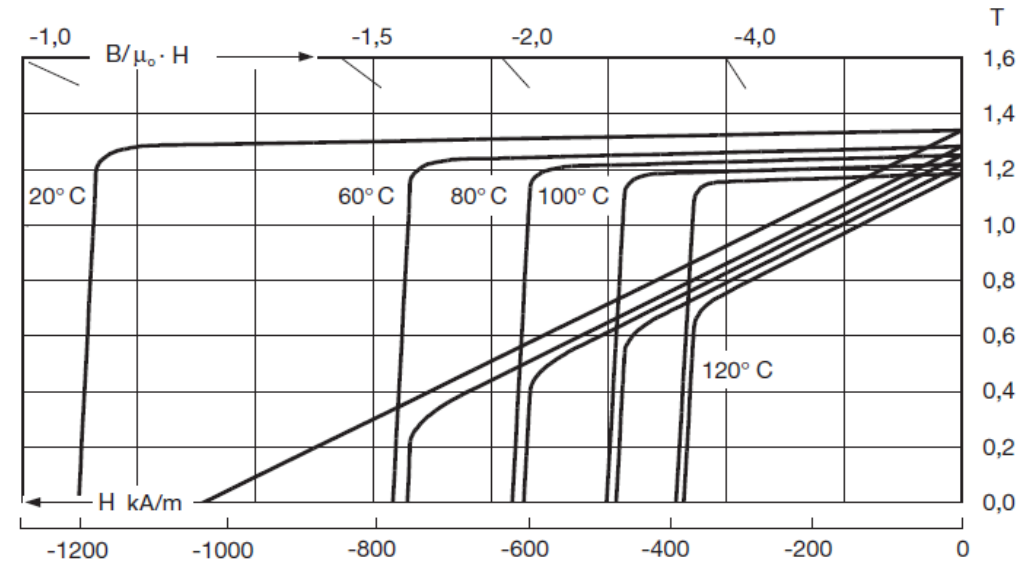

Figure 4.9 Typical demagnetization surface for sintered magnet on a Nd-Fe-B base, $\mathrm{B}(\mathrm{H})$ at different temperatures

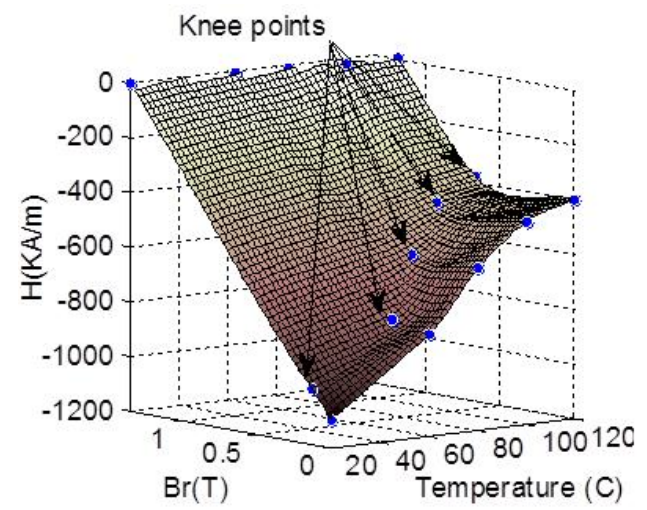

Figure 4.10 The closed form 3-D shape of the figure 4.9

\subsubsection{Calculations of transfer inductances}

In order to calculate $T_{W-M}$, equation (3-171), an artificial coil with one turn is placed in the PM sides where the normal surface vector of this coil is perpendicular to PM surface, see coils C1-C1' in figure 4.8(a). The surface area of this coil is $S_{\text {magnet }}$. This coil is better to be placed in the magnet flux barrier areas. The proposed model 
can be run at different snapshots of phase current, $I_{i}=\left(i_{d i}, i_{q i}\right)$, each for one electrical cycle. In equation (3-77), $T_{k}$ sets for one electrical cycle with respect to rotation speed and discrete rotation angle. Here the time step FE analysis is set to one electrical degree per $\mathrm{d} \tau$. The respective inductance of each phase for different current snapshots is then saved 3-D lookup Tables. A linear interpolation is used to interpolate the value of inductance at different rotor positions and snapshots of current break points. As presented in chapter 3, the voltage based inductance calculation method has two steps. The first and the second steps are respectively shown in figures 4.11 and 4.12. In this figure, iM1 represents the current to the coils $\mathrm{C} 1-\mathrm{C} 1$ '. $\mathrm{iM} 2$ is similar to iM1 for another artificial coil.

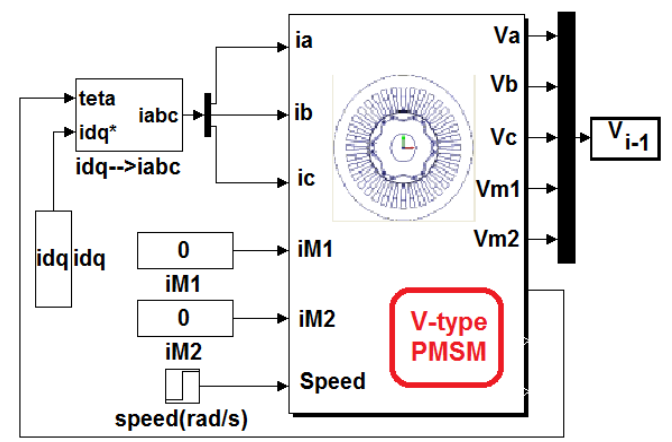

Figure 4.11 Inductance calculation subsystems, step 1

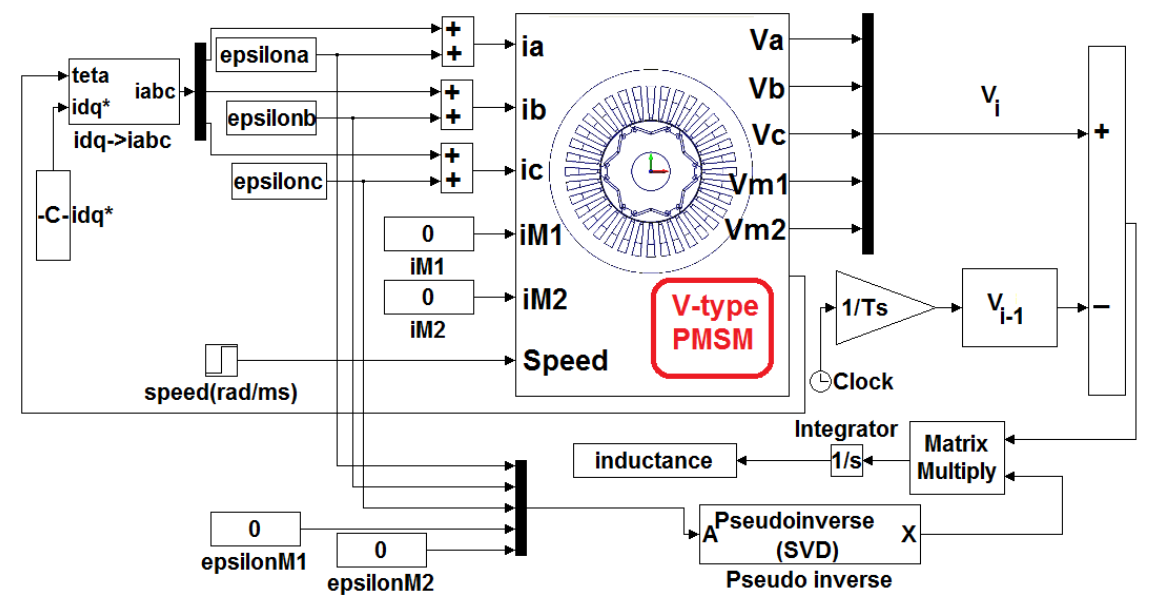

Figure 4.12 Inductance calculation subsystems, step 2 


\subsubsection{Calculation of operational PM temperature}

The basis of the used thermal model, figures 4-14 and 4-15, is presented at chapter 3. It is mentioned that before calculation of the conductive resistances, the equivalent geometry of the machine in which all the element part were aligned in radial direction in a form of a cylindrical slices should be calculated. This is particularly important in the rotor side. The rotor geometry conversion is done by assumption of equal surface area in real and equivalent model, compare figures 4.13 and 4.8(a). Comparing figures 4.13 and 4.8(a), $A_{e q}$ is the equivalent air gap area for the $A_{1}$ and $A_{2}, M_{e q}$ is the equivalent magnet area for the $M_{1}$ and $M_{2} \cdot M_{e q 3}$ and $M_{e q 4}$ are the equivalent magnet area for the $M_{3}$ and $M_{4}$ areas. The inputs to the thermal network are the active losses of the windings and the core losses. It is equivalent to the current source in thermal network. The outputs are the temperatures. It is equivalent to the voltages in the thermal network. $R_{a g}+R\left(A_{e q}\right)$ is the air gap thermal resistance. In figures 4.14 and 4.15 these two resistances are connected together. $\mathrm{A}_{\mathrm{g}}$ and $A_{e q}$ are the air gap area, $g$ is the air gap length and $K_{\text {air }}$ is the thermal conductivity of the air gap. The $R_{s t}, R_{s y}, R_{\text {case }}$ and $R_{\text {endcap }}$ are the conductive thermal resistances of the stator teeth, stator yoke, casing and the ending cap respectively. The $R\left(M_{e q}\right), R\left(S_{e q}\right)$, and $R\left(I_{e q 1}\right)$

to $R\left(I_{e q 6}\right)$ are the rotor resistances respectively, see figures 4.6 and 4.9.b. $R_{\text {endcap }}$ and $R_{a m b}$ are the respective convective resistance of the ending caps and the casing to the ambient environment, respectively. 


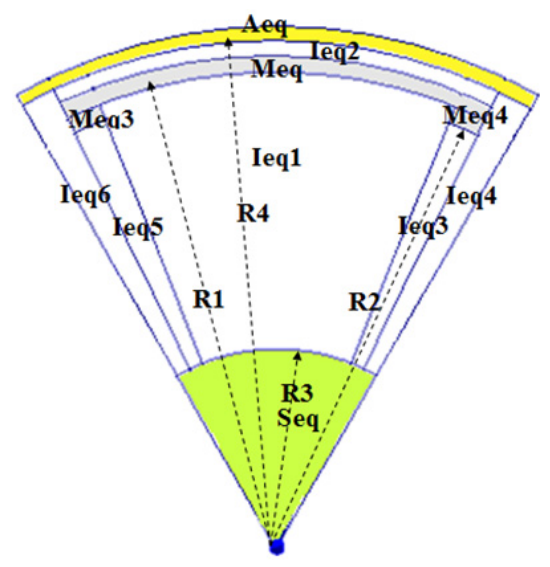

Figure 4.13 Geometry and dimension of the studied machine in (mm)

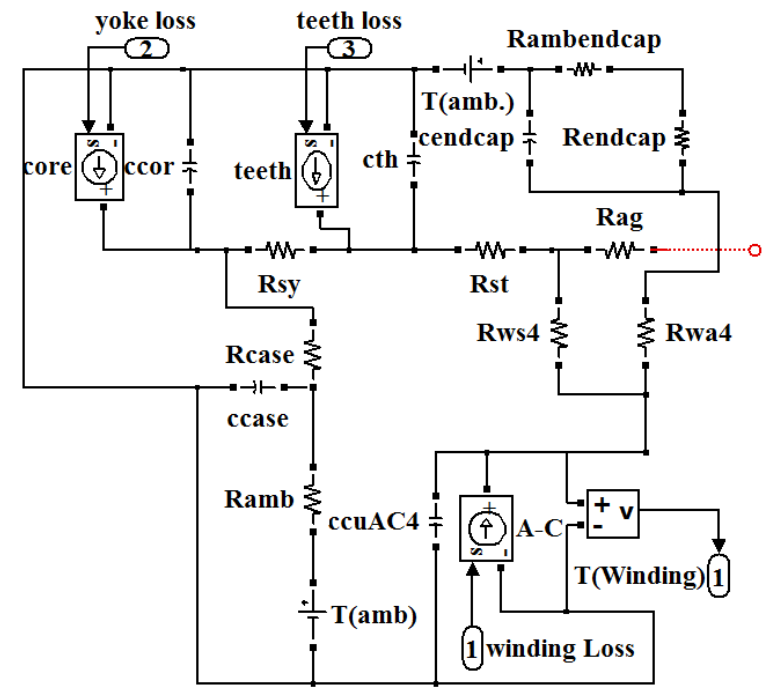

Figure 4.14 Transient thermal model of the stator

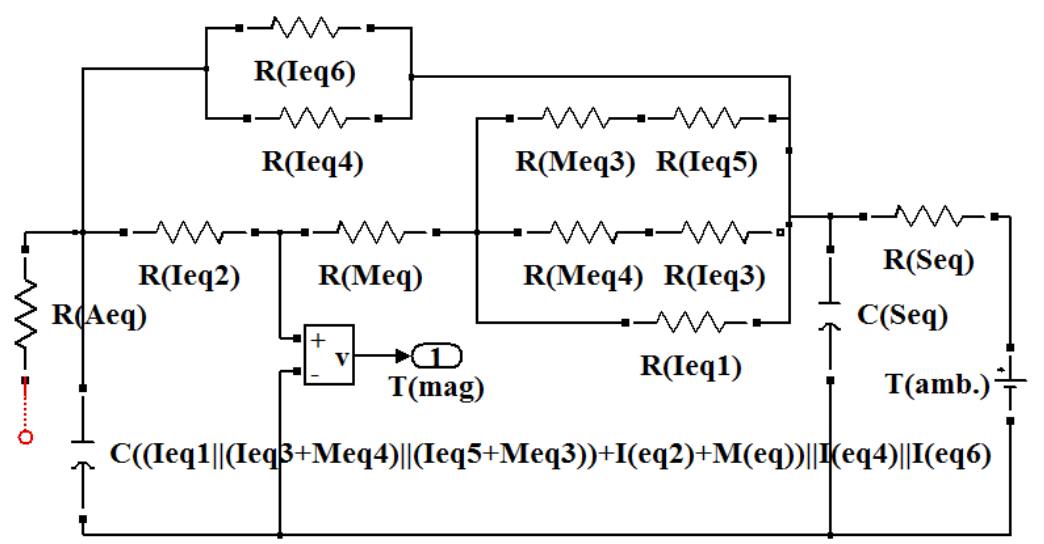

Figure 4.15 Transient thermal model of the rotor for the V-type PMSM 


\subsubsection{Simulation results}

Figure 4.16.a shows the $F_{T B}(T)$, equation (3-174). This is applied in equation (3173). Figure 4.16.b shows knee points as a function of temperature. This is applied in equation (3-177). Figure 4.16.c shows the average magnetic flux density on the face of the magnet along a line perpendicular to the flux path. Figure 4.16.d shows the minimum values for each curve depicted in figure 4.16.c. The reason that the minimum values are selected is to highlight where it is under higher demagnetization risk. Although the average remnanet flux density of magnets might be higher than the knee point, this assumption is made to prevent partial demagnetization. Figure 4.16.d is applied in the equation (3-175). The machine basic FE-based characteristics are illustrated in figure 4.17.a to 4.17.d. These values are collected in 1-D look up Tables as illustrated in Figure 3.16. Figure 4.18 shows the mutual inductance between the armature coil "a" and the artificial coil " $\mathrm{C} 1-\mathrm{C} 1$ "” located on flux barrier areas of $\mathrm{M}_{2}$. The mutual inductance between other phases and the "C1-C1"” are similar with 120 degree phase shift. These inductances create the $\mathrm{T}_{\mathrm{W}-\mathrm{M}}$ matrix in equation (3-171). Three 3-D look up Tables are used to collect these inductances.

Figure 4.19 and figure 4.20 indicate the armature self and mutual inductances respectively. These values together with other self and mutual inductances are employed in nine 3-D look-up Tables. The self and mutual inductance between other phases have 120 degree phase shift. All the inductances are calculated corresponding to similar rotor positions. From the inductance profile it is seen that the satutration not only change the value of inductances but it aslso shift the inductances. Also, it is seen that the satuation completely change the harmonic orders of self and mutual inductance profiles of phases. 


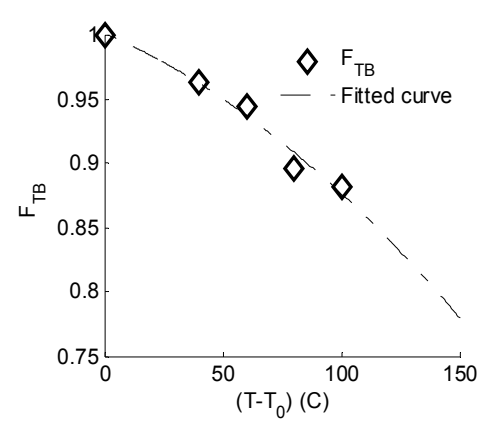

(a)
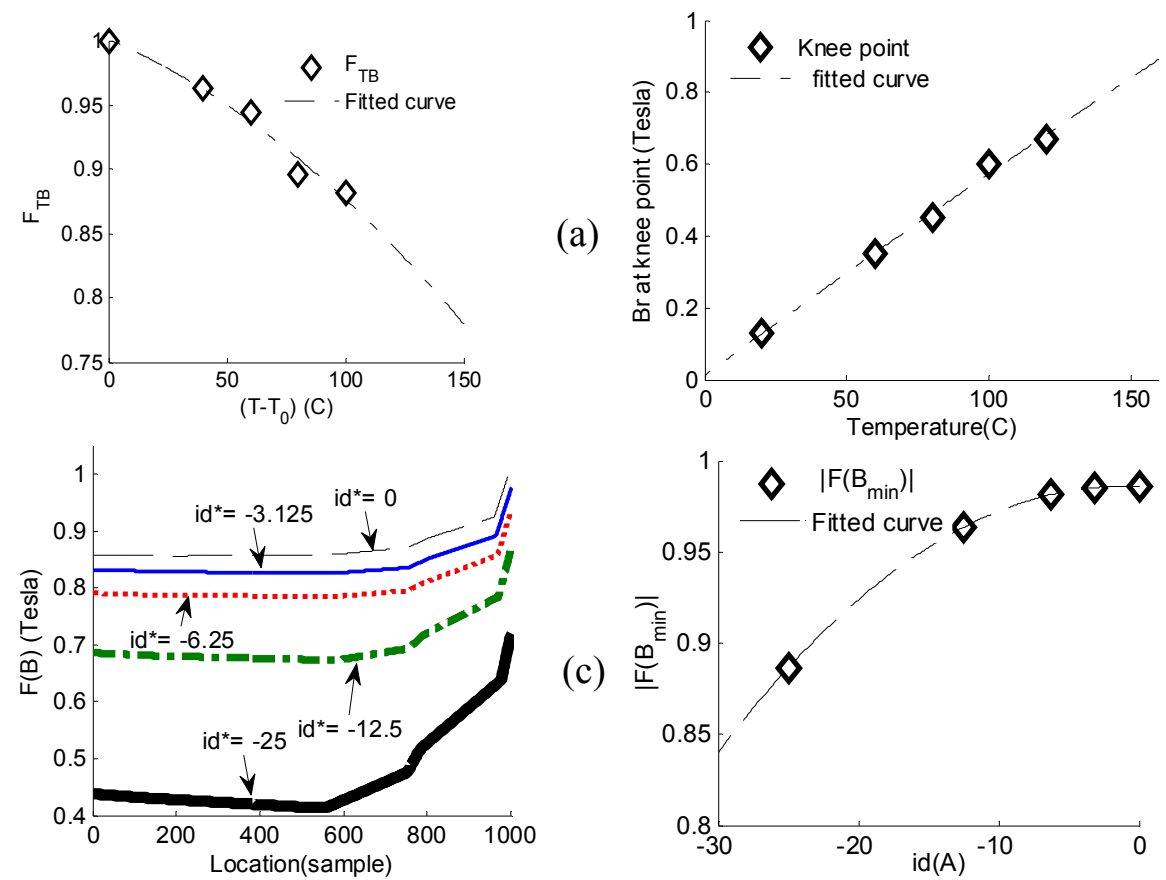

Figure 4.16 Relation between remnant flux density of the studied PM and temperature at $T_{0}=0^{\circ}(C), \mathrm{H}=0(\mathrm{~A} / \mathrm{m})(\mathrm{a})$, remnant flux density of magnet versus temperature at knee point (b) Flux density function on the face of magnet $M_{2}$ as a function of $i_{d}(\mathrm{c})$, Minimum flux density function on the face of $M_{2}$ as a function of $i_{d}(\mathrm{~d})$

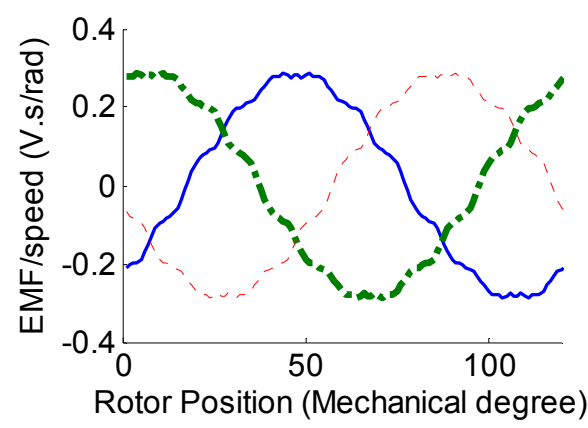

(a)

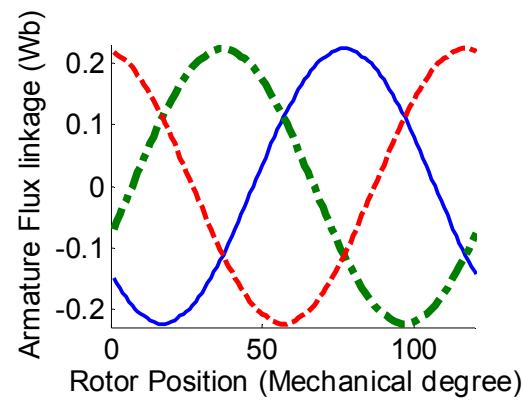

(b)
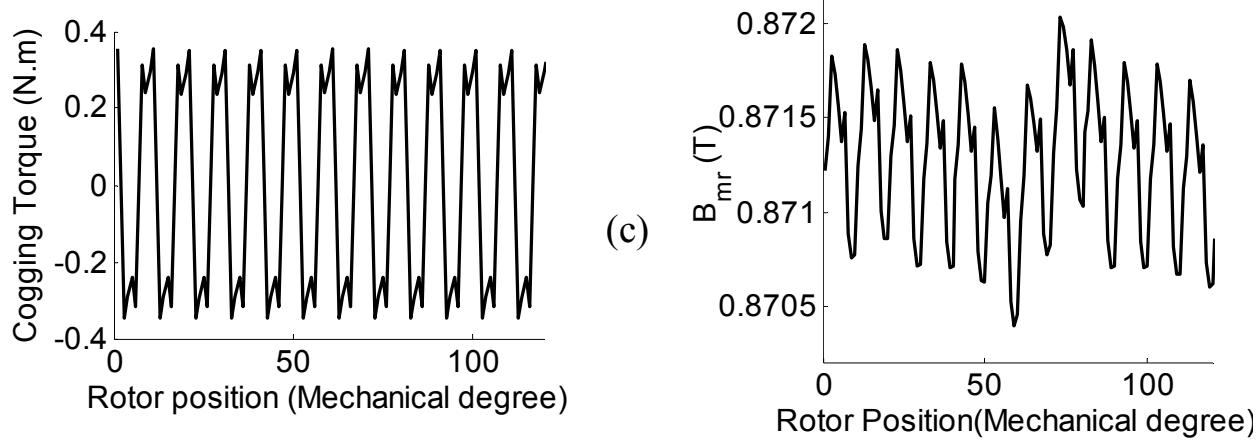

Figure $4.17 E_{a b c}(\mathrm{a}), \lambda_{R a b c}(\mathrm{~b}), T_{c o g}(\mathrm{c})$ and $B_{m r}(\mathrm{~d})$, calculated from a non-linear transient FE analysis with motion phase " $b$ " 


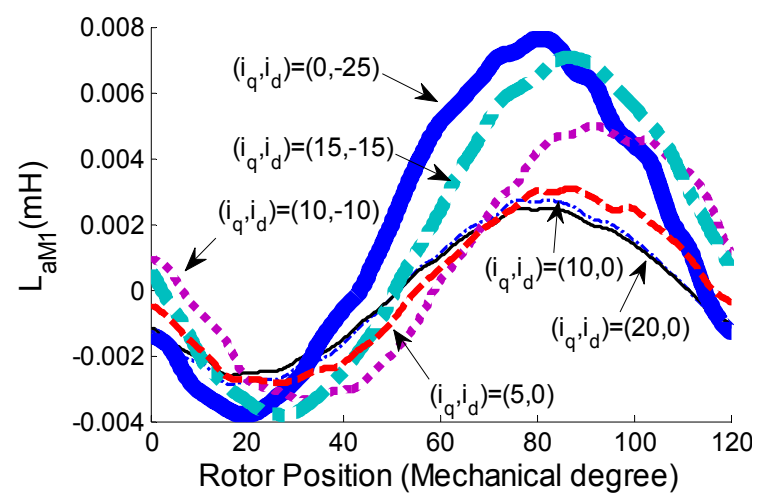

Figure 4.18 Mutual inductance between armature phase "a" and the magnet artificial coil C-C' on $\mathrm{M}_{2}$ at different current status

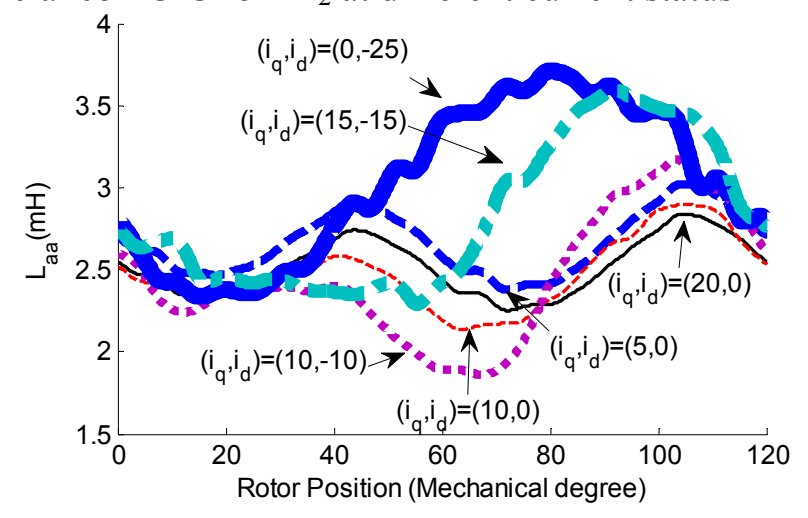

Figure 4.19 Self-inductance of armature phase "a" at different current status

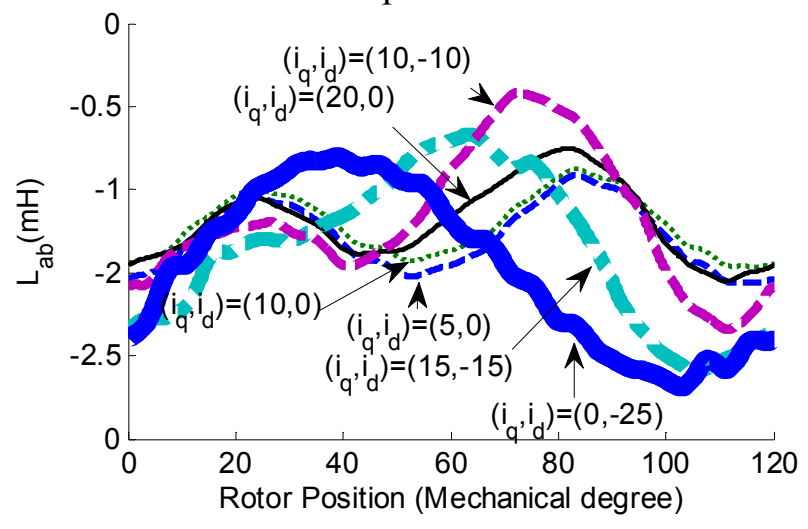

Figure 4.20 Mutual inductance between armature phase "a" and the armature

In order to evaluate the performance of the proposed flux weakening assessment and control, three scenarios are considered as:

Scenario 1: This scenario is designed to simulate the progressive and irreversible 
demagnetization during machine operation. In this case, the current limiter is deactivated. The progressive and irreversible demagnetization happens when the ambient temperature and absolute direct current, $i_{d}^{*}$, are constantly very high and, the load and speed reference are constant. For simulations, the ambient temperature sets to $T_{\text {ambient }}=60^{\circ}(C)$ for $\mathrm{t}>0(\mathrm{~s})$. The reference direct current, $i_{d}^{*}$, sets to $-25(\mathrm{~A})$ for $t>0$ (s). The load torque and the reference speed are $T_{L}=2(N . m)$ and $\omega_{m}=40(\mathrm{rad} / \mathrm{s})$ for $\mathrm{t}>0(\mathrm{~s})$. The response of the PMSM to scenario 1 for a $0.8(\mathrm{~s})$ simulation time is illustrated in figures 4.21.a through 4.21.i. Figure 4.21.a shows the movement of the magnetic operating point on the B-H curve of the magnet. Figure 4.21.b shows the movement of the knee point on the B-H curve of the magnet during operation. Figure 4.21.c shows the difference between figures 4.21.a and 4.21.b. Figures 4.21.d and 4.21.e show the EMF and the phase current of the machine during operation, respectively. Figures 4.21.f and 4.21.g show the torque and the speed of the machine during operation, respectively. The study of figures 4.21 reveals that without proper demagnetization control, the studied V-type PMSM will collapse in a short periods of time when it is working in a high ambient temperature with high absolute value $i_{d}^{*}$.

Scenario 2: This scenario is designed to simulate the physics of irreversible and not progressive demagnetization when the current limiter is not activated. For simulations, the ambient temperature sets to $T_{\text {ambient }}=60^{\circ}(C)$ for all $\mathrm{t}>0(\mathrm{~s})$. In this case, for $0<\mathrm{t}<0.3(\mathrm{~s})$, the $i_{d}^{*}$ sets to zero. For $0.3<\mathrm{t}<0.5(\mathrm{~s}), i_{d}^{*}$ sets to $-20(\mathrm{~A})$ and, for $\mathrm{t}>0.45(\mathrm{~s})$ it returns to zero again. The load torque and the reference speed respectively are $T_{L}=2(\mathrm{~N} . \mathrm{m})$ and $\omega_{m}=40(\mathrm{rad} / \mathrm{s})$. The response of the PMSM to scenario 2 for a 
0.8(s) simulation time is illustrated in figures 4.22.a to 4.22.i. Figure 4.22.a shows the movement of the magnetic operating point on the B-H curve of the magnet. Figure 4.22.b shows the movement of the knee point on the B-H curve of the magnet during operation. Figure 4.22.c shows the difference between figures 4.22.a and 4.22.b. Figures 4.22.a to 4.22.c reveal that, when the absolute $i_{d}^{*}$ is increased at $\mathrm{t}=0.3(\mathrm{~s})$ the magnet operating point drops below the knee point and irreversible demagnetization occurs; when the $i_{d}^{*}$ is set back to zero at $\mathrm{t}=0.5(\mathrm{~s})$, the operating point is unable to come back to its initial condition. Figures 4.22.d and 4.22.e show the EMF and the phase current of the machine during operation, respectively. Figures $4.22 \mathrm{f}$ and $4.22 . \mathrm{g}$ show the torque and the speed of the machine during operation, respectively. By studying of figures 4.22.d to 4.22.g is can be concluded that the main reason for increasing the phase current is the decrease of the EMF during a constant load and speed operation. The reason for EMF decrease is the irreversible demagnetization that is occurred during $0.3<\mathrm{t}<0.5(\mathrm{~s})$. Figures, 4.22.h and 4.22.i show the reference direct current and operational armature self and mutual inductances. The inductances are a function of rotor position and $\left(i_{d}, i_{q}\right)$.

Scenario 3: This scenario is exactly the same as the scenario 2 but the current limiter is activated to prevent irreversible demagnetization. The value of security margin, $B_{o}$, is set to 0.1 (Tesla). In this situation the value of the $i_{d}^{*}$ and $i_{q}^{*}$ are controlled by current limiter before transferring to current regulator. The comparisons of figures 4.22.a to 4.22.i with their respective figures of 4.23.a to 4.23.i prove that the current limiter could prevent irreversible demagnetization. This is performed by automatic regulation of $i_{d}^{*}$ shown in figure 4.23.h. 


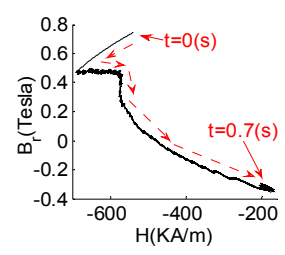

(a)

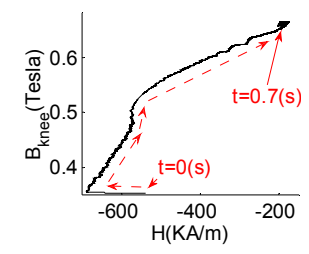

(d)

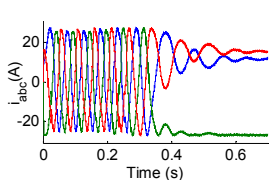

(g)

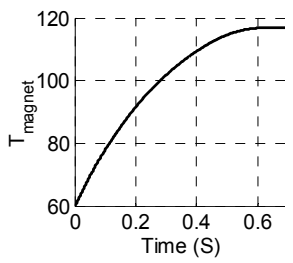

(b)

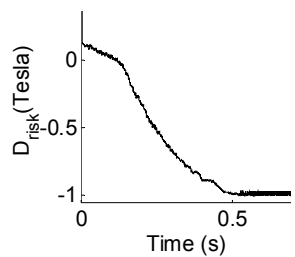

(c)

(e)

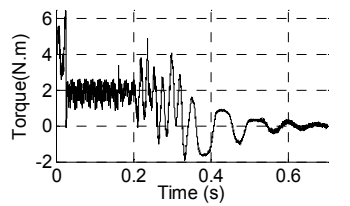

(f)

(h)

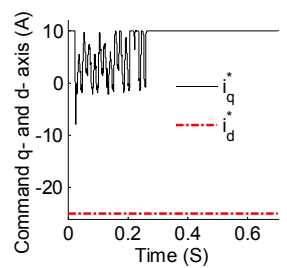

(c)

(b)

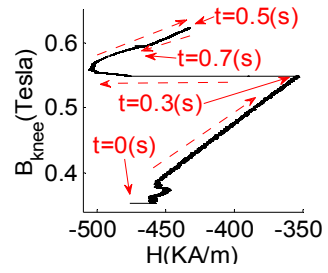

(e)

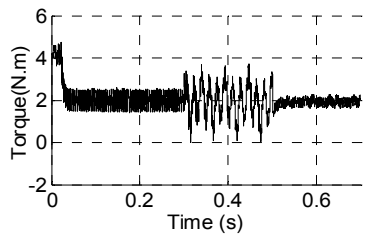

(f)

(h)

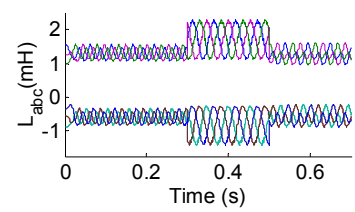

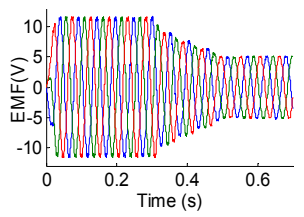

(d)

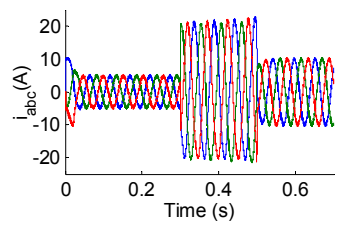

(g)

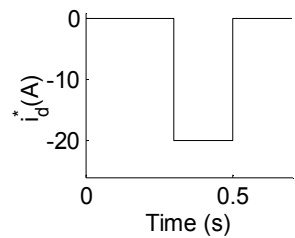

demagnetization, the current limiter is deactivated for all $t>0$ (s) 


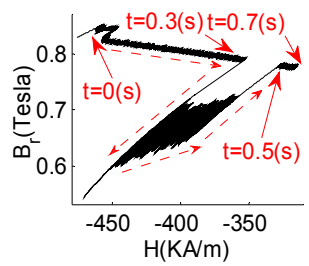

(a)

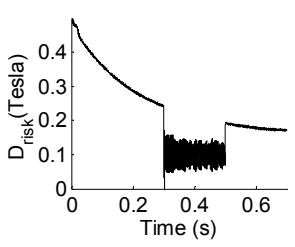

(d)

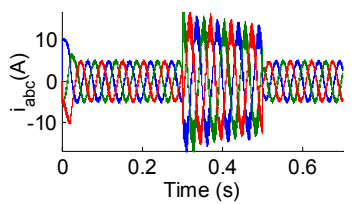

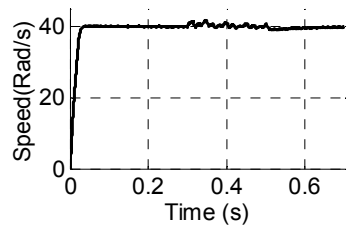

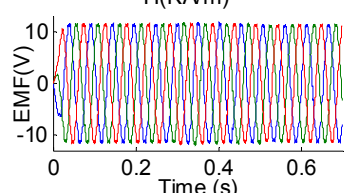

Figure 4.23

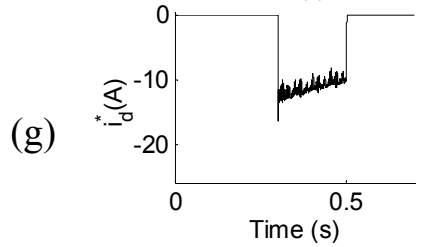

(b)

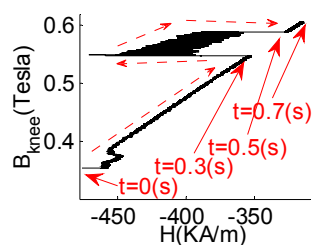

(e)

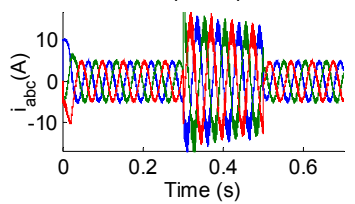

(h)

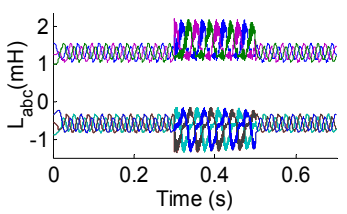

rreversible demagnetization, the current limiter is activated for all $\mathrm{t}>0$ (s)

Similar experiments are performed using the full time-stepping FE-analysis. The results of the developed model agree with that of the time-stepping FE model. The flux path for the respective currents of $\left(i_{d}^{*}, i_{q}^{*}\right)=(-25,0)$ and $\left(i_{d}^{*}, i_{q}^{*}\right)=(0,0)$ are shown in figure 4.24.a and 4.24.b respectively. The magnet temperature is $20^{\circ}(\mathrm{C})$. From 4.24(b) is observed that this amount of direct current is able to reduce the armature flux linkage is close to zero. The absolute flux density for the respective current $\left(i_{d}^{*}, i_{q}^{*}\right)=(-25,0)$ is shown in figure 4.25 . From this figure it is observed that the minimum flux density on the face of PM reaches $0.44[\mathrm{~T}]$ which proves the calculated results from analytical model.

In this PMSM design, the magnetic flux closes almost its entire path through magnets. It is the reason that the demagnetization and flux weakening are strongly related. Although, this high flux weakening capability in this design gives promising advantages for high speed and fault tolerance operation of PMSM, the design of realtime demagnetization control for this PMSM design is vital to prevent most probable 
demagnetization in highly variable-temperature operational environments. In addition, the developed current limiter gives more freedom to PMSM designers to reach to much more optimal designs without scarifying the magnet thickness and machine volume. It also ensures the PMSM drivers to steadily use the machine in harsh operational environments. The result has shown that without using a real-time demagnetization control the risk of demagnetization in a PMSM machine design with high flux weakening and demagnetization capability goes very high especially when the ambient temperature is high. Using the developed demagnetization control algorithm, the risk of PM demagnetization operating on harsh operational condition can be reduced.

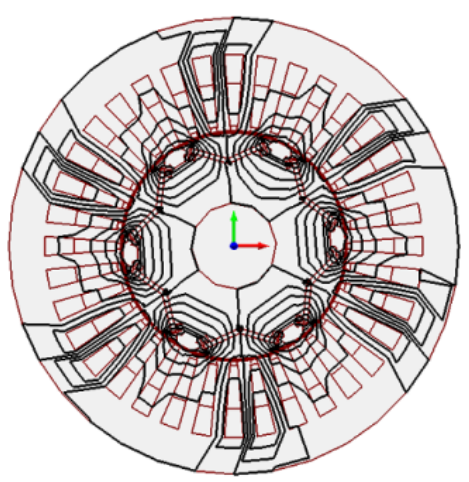

(a)

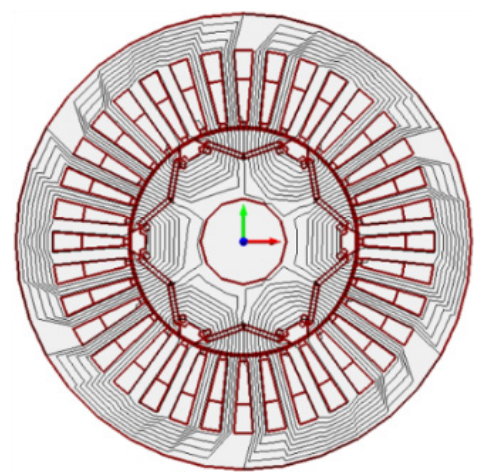

(b)

Figure 4.24 Absolute flux density on the magnets for the V-type PMSM for $\left(i_{d}^{*}\right.$, $\left.i_{q}^{*}\right)=(-25,0)$ and $T_{\text {magnet }}=20^{\circ}(C)$ and for $\left(i_{d}^{*}, i_{q}^{*}\right)=(0,0)$ and $T_{\text {magnet }}=20^{\circ}(C)$.

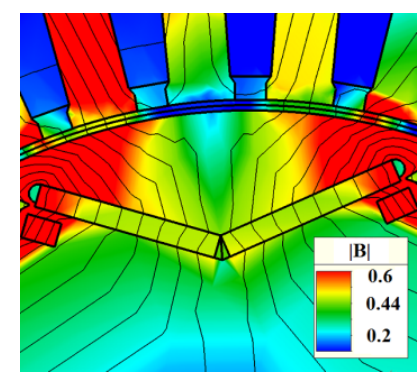

Figure 4.25 Magnetic flux picture (b) for the V-type PMSM for $\left(i_{d}^{*}, i_{q}^{*}\right)=(-25,0)$ and $T_{\text {magnet }}=20^{\circ}(C)$. 


\subsubsection{Demagnetization assessment in different PMSM designs}

In order to compare the demagnetization in different PMSM design, a flat-type PMSM and a U-type PMSM are designed. The back EMF- and the current density and the stators of these machine is similar to the V-type PMSM shown in figure 4.8. The rotors of these designs are shown in figure 4.26 and 4.27 and 4.28, respectively.

For comparison of the reaction of designs to the physics of irreversible and not the progressive demagnetization during PMSM-drive operation, initially, for $0<\mathrm{t}<0.3$ (s) the $i_{d}^{*}$ is set to zero. For $0.3<\mathrm{t}<0.45$ (s) it is -20 (A) and, for $\mathrm{t}>0.45$ (s) it sets to zero again. The load torque is $T_{L}=2(N \cdot m)$. The response of the PMSM to this scenario for a $0.8(\mathrm{~s})$ simulation time is illustrated in figures 4.29 and 4.30 .
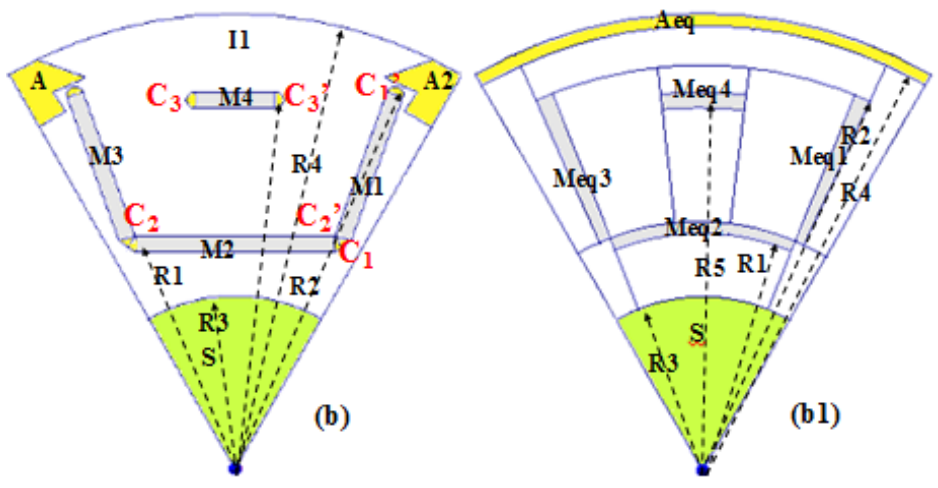

Figure 4.26 Rotor geometry of double PMSM, artificial coil C1-C1' to C4-C4' is located on flux barrier areas of $\mathrm{M}_{1}$ to $\mathrm{M}_{4}(\mathrm{~b})$, equivalent geometry of double PMSM (b1)

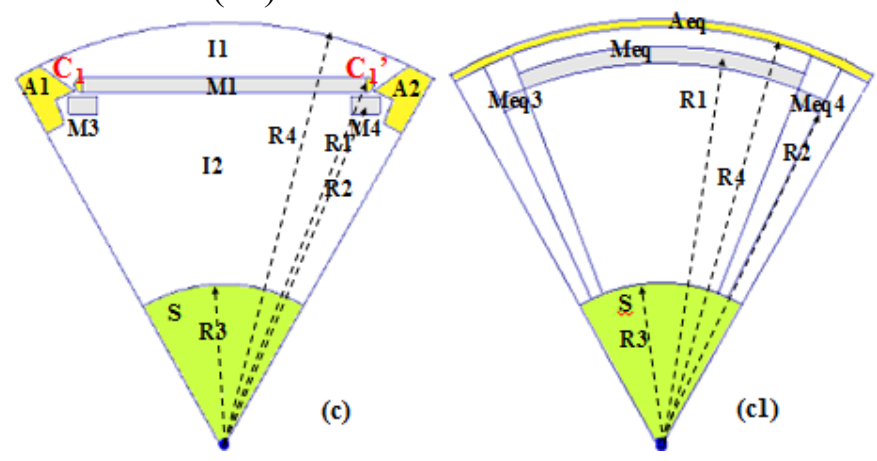

Figure 4.27 Rotor geometry of flat PMSM, artificial coil C1-C1' is located on flux barrier areas of $\mathrm{M}_{1}(\mathrm{c})$ : equivalent geometry of flat PMSM (c1) 


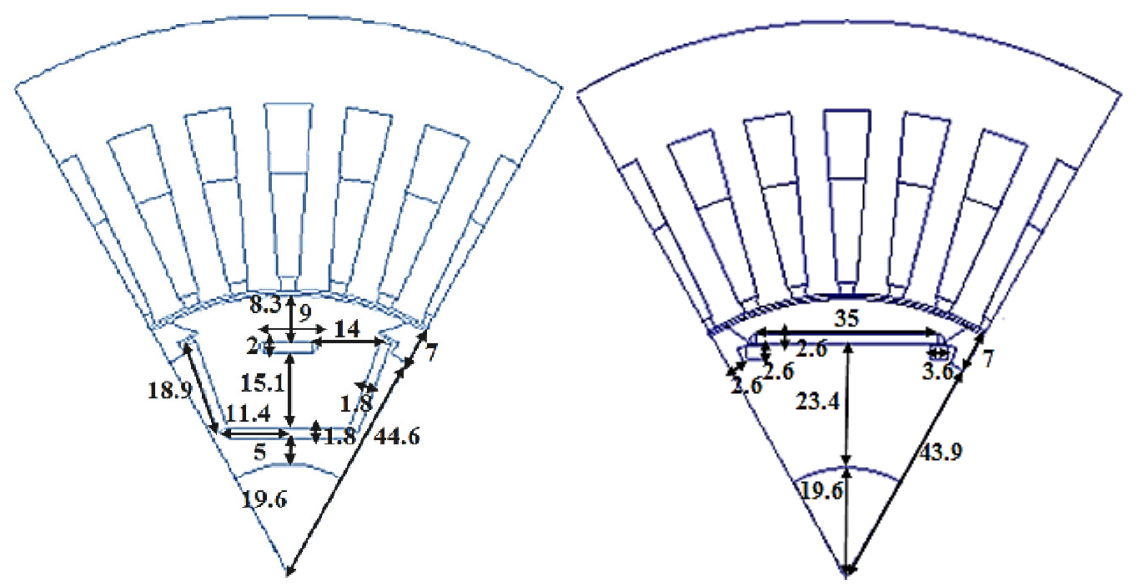

Figure 4.28 The dimension of the double-type and the flat PMSM

The operational B-H curves and the demagnetization risk function of the studied machines are shown in figure 4.29. It can be seen when the $i_{d}^{*}$ is increased at $\mathrm{t}=0.3(\mathrm{~s})$ the magnet operating point drops below the knee point and irreversible demagnetization occurs; when the $i_{d}^{*}$ is set back to zero at $\mathrm{t}=0.45(\mathrm{~s})$, the operating point is unable to come back to its initial condition. Figure 4.30 show the EMF and the $i_{q}^{*}$ in double, flat and V-type PMSM, respectively. By comparison of EMF and command q-axis current is can be concluded that the main reason for increasing the $i_{q}^{*}$ is the decrease of the EMF for a constant load and speed. The reason for EMF decrease is the irreversible demagnetization that occurred during $0.3<\mathrm{t}<0.45$ (s).
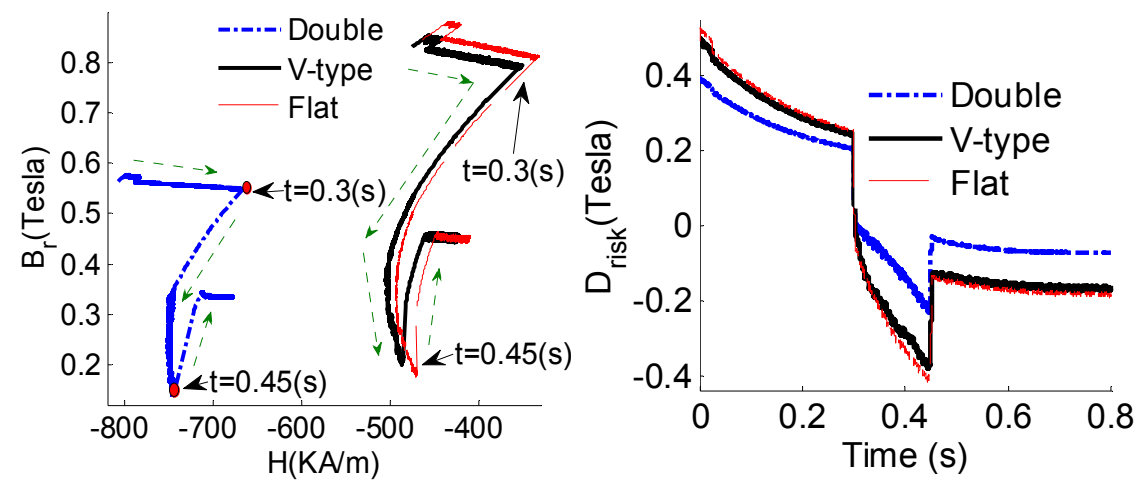

Figure 4.29 Irreversible and not progressive demagnetization 

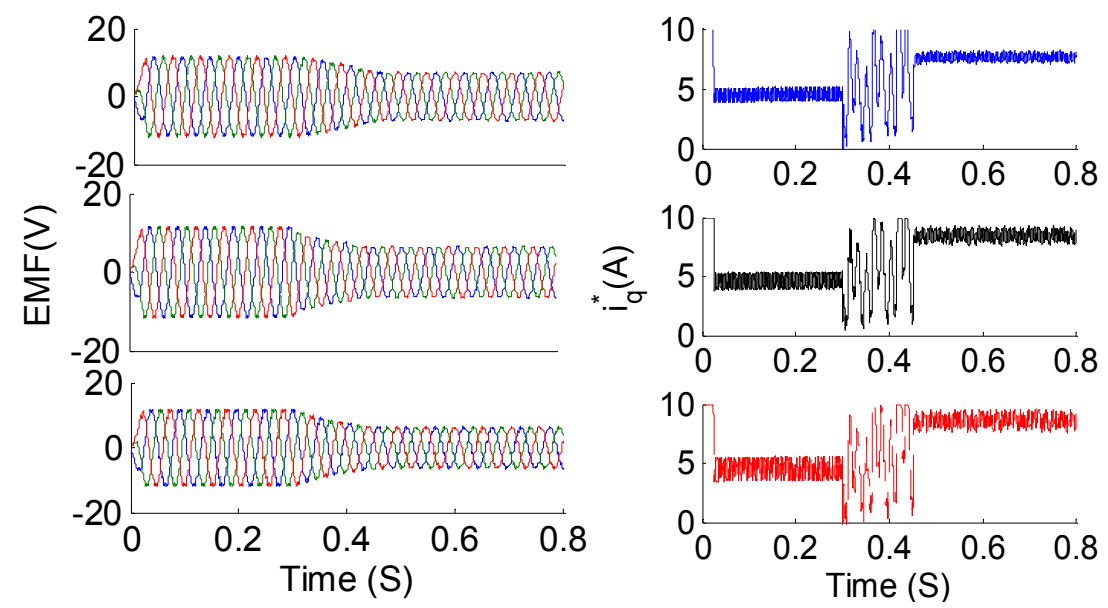

Figure 4.30 Irreversible and not progressive demagnetization

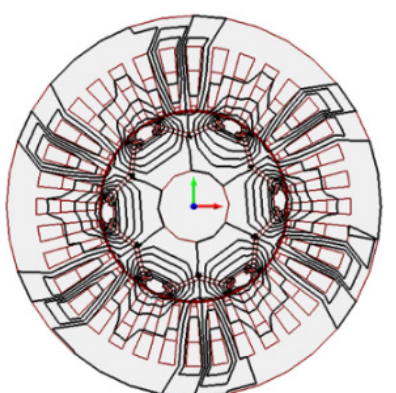

(a)

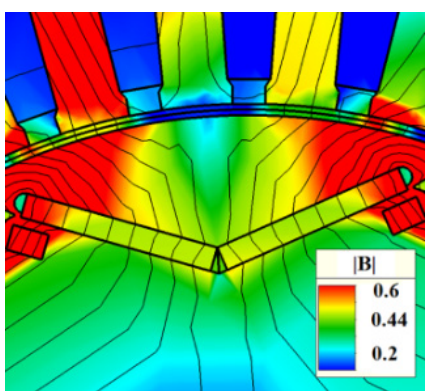

(a1)

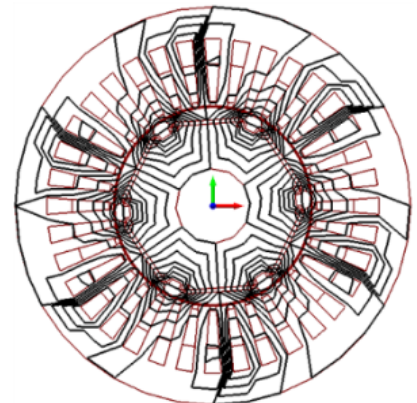

(b)

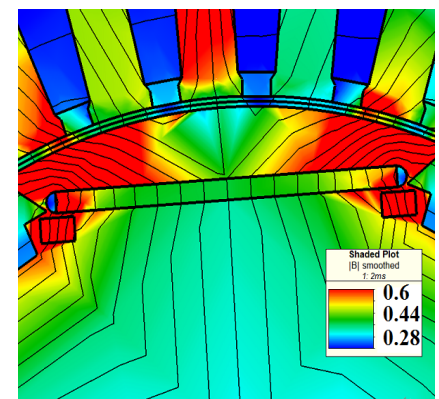

(b1)
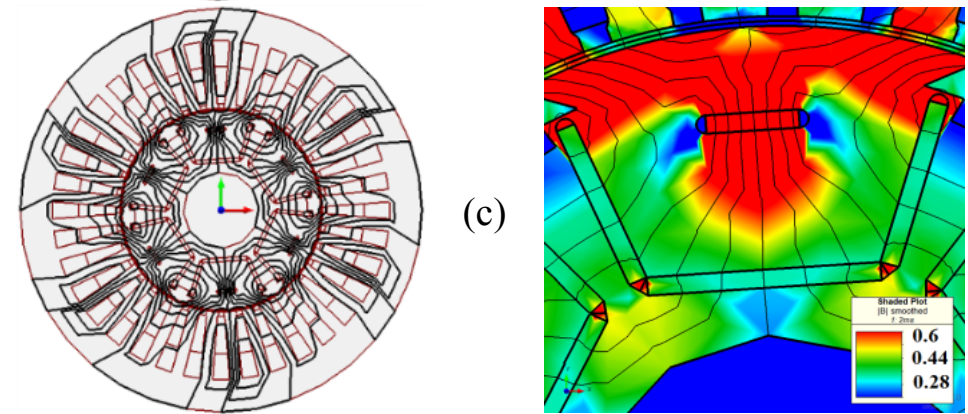

(c1)

Figure 4.1 Magnetic flux picture for the three PMSM in (a), (b) and (c) and absolute flux density on the magnets in (a1), (b1), (c1). All for the ( $\left.i_{d}^{*}, i_{q}^{*}\right)=(-25,0)$ and $\mathrm{T}_{\text {ambient }}=20^{\circ}(\mathrm{C})$ 
The flux path and the absolute flux density of the PMSM designs are shown in figure 4.30. The reference $\left(i_{d}^{*}, i_{q}^{*}\right)$ is $(-25,0)$ and the magnet temperature is $20^{\circ}(\mathrm{C})$. Form figures 4.31.a, 4.31.b and 4.31.c it is observed that this amount of direct current is able to reduce the armature flux linkage almost close to zero. Moreover, it is observed that for these PMSM designs, the magnetic flux closes almost its entire path through magnets. The double and the V-type designs are the worst and the best designs, respectively in terms of reverse demagnetization. The calculated results from the field domain verify the calculated results from analytical approach.

\subsection{Summary}

The current technologies in the PMSM drive systems were reviewed. The permanent magnet (PM) demagnetization control for optimal and reliable flux weakening control in permanent magnet synchronous machines (PMSM) is presented. A physics-based model was developed to estimate the magnetic operating point of the PM poles during the operation of PMSM. The model was enabled to dynamically estimate the average and the partial PM demagnetization due to reverse armature field as well as the PM over temperature. The model is a function of the physical geometry and material of the PMSM, physics of demagnetization and the ambient temperature. The demagnetization assessment and control were verified on a V-type PMSM using the modified FE-based coupled thermal-field-circuit phase variable model and the time step FE analysis. Three PMSM design were compared concerning demagnetization issue. It was observed that increasing the flux concentration factor in

the design of PMSM by enlarging the magnet contact area increase the risk of demagnetization. 


\section{Chapter 5 Sensorless Control of PM Synchronous Machines by Physics-based EMF Observer}

\subsection{Overview}

A sensorless rotor position estimation and speed control of three phase radial flux surface mounted permanent magnet synchronous machine (SMPMSM) is presented. A novel thermo-electric physics-based modeling approach was developed to evaluate more realistic information about the instantaneous behavior of the machine during operation. The extracted information from the physics-based models was employed in the back electromotive force (EMF) observer. The mathematical modeling, stability and transient analysis of the observer are developed and described in details. The sensorless operation of a $0.2(\mathrm{~kW})$ SMPMSM was verified numerically and experimentally for medium low, low and very low speeds at different operational contingencies. The results show that, in spite of significant machine model variations and increased noise level especially at very low speeds, the accuracy of estimated speed and rotor position using the developed observer are acceptable.

\subsection{Sensorless rotor position estimation and control in PMSM}

In general, the rotor position estimation techniques can be divided into two classes of model-based, non-model based (saliency based) methods. The Machine's EMF, flux linkage, or inductance estimation is the basis of model-based class. The signal processing of current response due to the high frequency voltage signal injection is the basis of the non-model based class which is suitable only for the PMSM with saliency (IPMSM) $[152,153]$. These methods can be found in the literature as flux estimation from the voltage model of PMSM[162]; inductance based estimators[163], extended Kalman filters[78], open loop or closed loop state observers[156,157], 
model reference adaptive schemes [166], artificial intelligence-based estimators [167] and sliding mode observers [75].

Frequently used methods for rotor position estimation of a SMPMSM at medium and high speed is established through the model-based EMF observers [168]. Usually the EMF is observed by simple open loop observers. However, these suffer from several disadvantages. These include simultaneous adjustments of the observer and speed controller, unwanted machine model variations due to the temperature, saturation, flux linkage harmonics as well as the interaction of the speed PI controller and observer especially at low speeds. Indeed, the domination of resistive and inductive voltage drops at low speeds [168] prevents the usage of these simple observers.

To resolve the problem of open loop observers especially at low speeds, several methods were used. These include a combination of the model-based and non-model based methods [169] or the use of close loop observers which are the extended version of the open loops observers[164]. In closed loop observers, the problem of stator resistance mismatches or even the machine parameter mismatch or system noise was resolved for low speed applications. However, these have higher computational complexity and also these are almost mode selective with respect to different speeds[164]. In general, the inconsistency of both open loop and closed loop observers was that the observer must be faster than the observed system. However it should be slow enough to suppress underestimated electrical noise and mechanical vibrations. Concerning this puzzle, the employment of linear low pass filters with fixed cut-off frequency for a wide speed range was under serious limitation.

This chapter presents a position estimation and sensorless speed control of SMPMSM using a physics-based observer. The physics-based observer was created 
by the combination of the physics-based model of the machine and a hysteresis estimator. The physics-based model of the machine was created from the phasevariable saturation dependent inductances, temperature-dependent winding resistance and the EMF. The EMF was calculated by hysteresis estimator based on the difference of the measured and estimated currents from the real machine and the observer, respectively. The inductance was calculated from an offline non-linear transient finite element analysis with motion and retrieved by look up Tables inside the observer. The real time winding temperature was estimated from an embedded transient lumped thermal network obtained from the geometry of the machine and material properties. During angle calculations, the underestimated electrical noises are canceled out using an adaptive angle compensation system. The developed sensorless speed control algorithm was implemented on a $0.2 \mathrm{~kW}$ SMPMSM machine using the hardware interface (dSPACE1103). The rotor position estimation and speed controllability over a medium to very low speed band are studied. In addition, the transient response and robustness of machine-drive system with respect to load and voltage disturbances are discussed in detail.

\subsection{Physics-based sensorless control of PMSM}

For design of the physics-based sensorless control, the physics-based model of the machine was used to design a EMF observer. The line-line thermoelectric electrical modeling of a 3-phase PMSM ignoring demagnetization and without access to the neutral point can be described as:

$$
\begin{aligned}
{\left[\begin{array}{l}
i_{a}(t) \\
i_{b}(t)
\end{array}\right]=} & A\left(\theta(\tau), i_{d}(\tau), i_{q}(\tau)\right)^{-1} \times \\
& \int_{0}^{t}\left\{B(T(\tau)) \cdot\left[\begin{array}{c}
i_{a}(\tau) \\
i_{b}(\tau)
\end{array}\right]+\left[\begin{array}{c}
V_{a b}(\tau) \\
V_{b c}(\tau)
\end{array}\right]+\left[\begin{array}{c}
E_{b}(\theta(\tau))-E_{a}(\theta(\tau)) \\
E_{c}(\theta(\tau))-E_{b}(\theta(\tau))
\end{array}\right]\right\} \cdot d \tau \\
i_{a}+i_{b}+i_{c}= & 0
\end{aligned}
$$


where

$$
\begin{aligned}
& A\left(\theta, i_{d}, i_{q}\right)=\left[\begin{array}{cc}
L_{a a}-L_{a c}-L_{b a}+L_{b c} & L_{a b}-L_{a c}-L_{b b}+L_{b c} \\
L_{b a}-L_{a c}-L_{b c}+L_{c c} & L_{b b}-L_{b c}+L_{c c}-L_{b c}
\end{array}\right] \\
& B(T)=\left[\begin{array}{cc}
-r_{a}(T) & r_{b}(T) \\
-r_{c}(T) & -r_{b}(T)-r_{c}(T)
\end{array}\right]
\end{aligned}
$$

where $\mathrm{a}, \mathrm{b}$ and $\mathrm{c}$ are phase indices. $V_{a b}, V_{b c}$ are the line-line terminal voltage of the machine. Here, $E$ is the phase side EMF. $\mathrm{i}_{\mathrm{a}}$ and $\mathrm{i}_{\mathrm{b}}$ are the phase currents.

In the above equation, $T$ is the temperature of the windings, $\tau$ is the integration variable, $\theta$ is the rotor position. The real inductance matrix, $A$, is a function of $\left(\theta, i_{d}, i_{q}\right) \cdot\left(i_{d}, i_{q}\right)$ indicates the saturation level. The real resistance matrix, $B$, is a function of temperature. In chapter 3 the calculations of inductances and the dependence of the resistances to temperature were explained, comprehensively.

The hysteresis estimator for EMF calculation was designed based on a stator current tracking scheme. Due to the fact that only the stator currents are directly measurable in a PMSM drive, the hysteresis band was selected on the real stator current trajectory. In this way, when the difference between the actual and estimated currents become lower or higher than a hysteresis band, a positive or negative value is added to the EMF in a very short periods of time. This is to force the current estimation error to become zero and the estimated currents track the measured ones regardless of any disturbances and uncertainties of the drive system, see figure 5.1. The physics-based EMF Observer was designed similar to the line-line machine voltage equations of PMSM as: 


$$
\begin{aligned}
& {\left[\begin{array}{l}
\hat{i}_{a}(t) \\
\hat{i}_{b}(t)
\end{array}\right]=\hat{A}\left(\theta(\tau), i_{d}(\tau), i_{q}(\tau)\right)^{-1} \times} \\
& \int_{0}^{t}\left\{\hat{B}(T(\tau)) \cdot\left[\begin{array}{l}
\hat{i}_{a}(\tau) \\
\hat{i}_{b}(\tau)
\end{array}\right]+\left[\begin{array}{c}
V_{a b}^{*}(\tau) \\
V_{b c}^{*}(\tau)
\end{array}\right]+\left[\begin{array}{l}
\hat{E}_{b}(\hat{\theta}(\tau))-\hat{E}_{a}(\hat{\theta}(\tau)) \\
\hat{E}_{c}(\hat{\theta}(\tau))-\hat{E}_{b}(\hat{\theta}(\tau))
\end{array}\right]\right\} \cdot d \tau \\
& \hat{i}_{a}+\hat{i}_{b}+\hat{i}_{c}=0
\end{aligned}
$$

where

$$
\begin{aligned}
& \hat{A}\left(\hat{\theta}, i_{d}, i_{q}\right)=\left[\begin{array}{ll}
\hat{L}_{a a}-\hat{L}_{a c}-\hat{L}_{b a}+\hat{L}_{b c} & \hat{L}_{a b}-\hat{L}_{a c}-\hat{L}_{b b}+\hat{L}_{b c} \\
\hat{L}_{b a}-\hat{L}_{a c}-\hat{L}_{b c}+\hat{L}_{c c} & \hat{L}_{b b}-\hat{L}_{b c}+\hat{L}_{c c}-\hat{L}_{b c}
\end{array}\right] \\
& \hat{B}(\hat{T})=\left[\begin{array}{ll}
-r_{a}(\hat{T}) & r_{b}(\hat{T}) \\
-r_{c}(\hat{T}) & -r_{b}(\hat{T})-r_{c}(\hat{T})
\end{array}\right] \\
& {\left[\begin{array}{l}
\hat{E}_{a} \\
\hat{E}_{b} \\
\hat{E}_{c}
\end{array}\right]=\frac{a_{i} \cdot V_{d c}}{2} \cdot\left[\begin{array}{l}
\hat{E}_{a 0} \\
\hat{E}_{b 0} \\
-\hat{E}_{b}-\hat{E}_{a}
\end{array}\right]+\frac{a_{i} \cdot g \cdot V_{d c} \cdot s}{2\left(\left(s / \omega_{l}\right)+1\right)} \cdot\left[\begin{array}{l}
\hat{E}_{a 0} \\
\hat{E}_{b 0} \\
-\hat{E}_{b}-\hat{E}_{a}
\end{array}\right]}
\end{aligned}
$$

In the above equations, $\hat{A}$ is the equivalent inductance matrix, $\hat{B}$ is the equivalent resistance matrix and $\left(i_{d}, i_{q}\right)$ is the equivalent saturation level. $V_{d c}$ is the fixed DC voltage supplying the fully controlled inverter. The superscript '*' denotes a command variable. The hat "^" indicates the estimated variables. The vectors $\hat{E}_{a 0}$ and $\hat{\mathrm{E}}_{\mathrm{b} 0}$ are defined as:

$$
\left\{\begin{array}{l}
\hat{E}_{a 0}=1 \forall\left(\hat{i}_{a}-i_{a}\right)>h,-1 \forall\left(\hat{i}_{a}-i_{a}\right)<-h, 0 \forall\left|i_{a}-\hat{i}_{a}\right|<h \\
\hat{E}_{b 0}=1 \forall\left(\hat{i}_{b}-i_{b}\right)>h,-1 \forall\left(\hat{i}_{b}-i_{b}\right)<-h, 0 \forall\left|i_{b}-\hat{i}_{b}\right|<h
\end{array}\right.
$$

where $h>0$ is the hysteresis band, $\omega_{l}$ is the cutoff frequency of the high pass filter. The $\omega$ must be sufficiently small to preserve the necessary component but large enough to eliminate the low-frequency component. The proper choice of $\omega_{l}$, helps to diminish the effect of the large hysteresis band and to diminish the unwanted effects 
due to the difference between reference and the actual voltage. By subtraction of equation (5-5) from equation (3-170) and assuming $V_{a b}=V_{a b}^{*}, V_{b c}=V_{b c}^{*}$, $A\left(\theta, i_{d}, i_{q}\right)=\hat{A}\left(\hat{\theta}, i_{d}, i_{q}\right), B(T)=\hat{B}(\hat{T})$ and $y \approx 1$, the motion equation is expressed as:

$$
\dot{M}=A\left(\theta, i_{d}, i_{q}\right) \cdot\left\{\begin{array}{l}
B(T) \cdot M-\frac{d A\left(\theta, i_{d}, i_{q}\right)}{d t} \cdot \frac{d \theta}{d t} \cdot M-\frac{d A\left(\theta, i_{d}, i_{q}\right)}{d\left(i_{d}, i_{q}\right)} \cdot \frac{d\left(i_{d}, i_{q}\right)}{d t} \cdot M \\
+\left[\begin{array}{l}
\hat{E}_{a}-E_{a}-\hat{E}_{b}+E_{b} \\
\hat{E}_{b}-E_{b}-\hat{E}_{b}+E_{b}
\end{array}\right]
\end{array}\right\}
$$

where motion, $M$, is defined as:

$$
M=\left[\begin{array}{l}
i_{a} \\
i_{b}
\end{array}\right]-\left[\begin{array}{l}
\hat{i}_{a} \\
\hat{i}_{b}
\end{array}\right]
$$

If the hysteresis band, $h>0$, is small enough and $A\left(\theta, i_{d}, i_{q}\right)$ in boundary of $t-d \tau<t<t+d \tau$ is constant then $\dot{M} \cdot M \leq 0$ and the physics-based model was synchronized with the actual machine and as a result:

$$
\left[\begin{array}{l}
\hat{E}_{a}-E_{a}-\hat{E}_{b}+E_{b} \\
\hat{E}_{b}-E_{b}-\hat{E}_{b}+E_{b}
\end{array}\right]=\left[\begin{array}{l}
0 \\
0
\end{array}\right]
$$

Because in (5-9) we chose, $\hat{E}_{c}=-\hat{E}_{b}-\hat{E}_{a}$ and assuming $E_{c}=-E_{b}-E_{a}$, the explicit solution was calculated as:

$$
\left[\begin{array}{c}
\hat{E}_{a} \\
\hat{E}_{b}
\end{array}\right]=\left[\begin{array}{c}
E_{a} \\
E_{b}
\end{array}\right]
$$

By converting equation (5-14) to stationary frame of reference we have:

$$
\left[\begin{array}{l}
\hat{E}_{\alpha} \\
\hat{E}_{\beta}
\end{array}\right]=\left[\begin{array}{rrr}
\cos (\theta) & \cos \left(\theta-\frac{2 \pi}{3}\right) & \cos \left(\theta-\frac{4 \pi}{3}\right) \\
-\sin (\theta) & -\sin \left(\theta-\frac{2 \pi}{3}\right) & -\sin \left(\theta-\frac{4 \pi}{3}\right)
\end{array}\right] \cdot\left[\begin{array}{c}
\hat{E}_{a} \\
\hat{E}_{b} \\
\hat{E}_{c}
\end{array}\right]
$$


where $\theta$ is the constant reference rotor position which was calculated from equation $(5-22)$.

\subsubsection{Adaptive angle compensator}

An adaptive angle compensator was designed. This increases the robustness of the proposed physics-based sensorless rotor position estimation and furthermore, helps to remove the remaining high frequency noise during angle calculations. It was mentioned that, the observer band width may miss by direct usage of linear low pass filters. For design of this system, the assumption was that the estimated EMF phasors in stationary frame of reference should always be perpendicular to each other. This is

equal to $\dot{\hat{E}}_{\alpha}=-\omega \cdot \hat{E}_{\beta}$ and $\dot{\hat{E}}_{\beta}=\omega \cdot \hat{E}_{\alpha}$. The estimated EMF can be compensated to always satisfy these criteria during angle calculations. From these two criteria, the linear compensation equations are defined in stationary $(\alpha-\beta)$ plane using derivation definition as:

$$
\begin{aligned}
& \dot{\hat{E}}_{\alpha}=-\omega \cdot \hat{\hat{E}}_{\beta}-O_{g}\left(\omega_{r e f}\right) \cdot\left(\hat{\hat{E}}_{\alpha}-\hat{E}_{\alpha}\right) \\
& \dot{\hat{E}}_{\beta}=\omega \cdot \hat{\hat{E}}_{\alpha}-O_{g}\left(\omega_{r e f}\right) \cdot\left(\hat{\hat{E}}_{\beta}-\hat{E}_{\beta}\right)
\end{aligned}
$$

where $\mathrm{O}_{\mathrm{g}}$ is the positive variable observer gain as a function of reference speed, $\omega_{\text {ref }}$. Here, $\hat{\hat{E}}_{\alpha}$ is the compensated $\hat{E}_{\alpha}$ and $\hat{\hat{E}}_{\beta}$ is the compensated $\hat{E}_{\beta}$. In order to consider the stability of the adaptive part the positive Lyapunov function which is defined as:

$$
V=\left(\hat{\hat{E}}_{\alpha}-\hat{E}_{\alpha}\right)^{2}+\left(\hat{\hat{E}}_{\beta}-\hat{E}_{\beta}\right)^{2}+A_{l}\left(\omega_{r e f}\right) \cdot(\hat{\omega}-\omega)^{2}
$$

where $\hat{\hat{E}}_{\alpha}-\hat{E}_{\alpha}, \hat{\hat{E}}_{\beta}-\hat{E}_{\beta}$ and $\hat{\omega}-\omega$ are the dynamic errors and $A_{l}\left(\omega_{\text {ref }}\right)$ is the positive 
variable which is a function of reference speed. To satisfy the Lyapunov stability criterion, $V^{T} \cdot \dot{V}<0$, we should have $\dot{V}<0$ that means:

$$
\begin{aligned}
\dot{V}= & -O_{g}\left(\omega_{r e f}\right) \cdot\left(\left(\hat{\hat{E}}_{\alpha}-\hat{E}_{\alpha}\right)^{2}+\hat{E}_{\beta}^{2}\right)+(\hat{\omega}-\omega) \\
& \times\left(-\left(\hat{\hat{E}}_{\alpha}-\hat{E}_{\alpha}\right) \cdot \hat{E}_{\beta}+\left(\hat{\hat{E}}_{\beta}-\hat{E}_{\beta}\right) \cdot \hat{E}_{\alpha}+A_{l}\left(\omega_{r e f}\right) \cdot(\dot{\hat{\omega}}-\dot{\omega})\right)
\end{aligned}
$$

If we assume that the speed varies slowly with respect to electrical constraints i.e. $\dot{\omega}=0$, then in order to make $\dot{V}<0$, we should have:

$$
\left(-\left(\hat{\hat{E}}_{\alpha}-\hat{E}_{\alpha}\right) \cdot \hat{E}_{\beta}+\left(\hat{\hat{E}}_{\beta}-\hat{E}_{\beta}\right) \cdot \hat{E}_{\alpha}+A_{l}\left(\omega_{r e f}\right) \cdot(\dot{\hat{\omega}}-\dot{\omega})\right)=0
$$

By simplification of equation (5-20), the adaptive compensation law was calculated as:

$$
(\dot{\hat{\omega}}-\dot{\omega})=\hat{\omega}=\frac{\left(\hat{\hat{E}}_{\alpha}-\hat{E}_{\alpha}\right) \cdot \hat{E}_{\beta}-\left(\hat{\hat{E}}_{\beta}-\hat{E}_{\beta}\right) \cdot \hat{E}_{\alpha}}{A_{l}\left(\omega_{\text {ref }}\right)}
$$

For proper dynamic response of the adaptive angle compensator, the gains, $A_{l}\left(\omega_{r e f}\right)$ and $O_{g}\left(\omega_{r e f}\right)$ should be chosen properly. By choosing smaller value for the $O_{g}\left(\omega_{\text {ref }}\right)$, the higher order harmonics was eliminated when the $A_{l}\left(\omega_{\text {ref }}\right)$ was small enough. The value of $A_{l}\left(\omega_{\text {ref }}\right)$ should be chosen properly in order to prevent the premature saturation of the integrators or adding extra delay in the system. The Simulink implementation of the adaptive angle compensator was illustrated in figure

\section{2 .}

Following angle compensation, the rotor position angle can be estimated as:

$\theta=a \tan 2=\left(\hat{\hat{E}}_{\alpha} / \hat{\hat{E}}_{\beta}\right), \theta=0 \forall \frac{d \theta_{r}}{d t}>0, \theta=\frac{2 \pi}{3} \forall \frac{d \theta_{r}}{d t}<0$

In order to prevent the chattering problem in a digital control system with finite 
switching frequency, a linear mean using Pade approximation [170] was added to the estimation approximation path, see figure 5.3. The Pade approximation is a rational function that can be thought of as a generalization of a Taylor polynomial. A rational function is the ratio of polynomials. Because these functions only use the elementary arithmetic operations, they are very easy to evaluate numerically. The mathematical description of this approximation is:

$$
\text { Output }=\frac{1-e^{-T_{d} \cdot s}}{T_{r} \cdot s} \cdot \text { Input }
$$

where $T_{r}$ determines the running window width in frequency domain, $T_{d}$ is the deadtime and $s$ is the Laplace operator.

\subsubsection{Stability of the observer}

With the positive definite function like $V=\frac{S^{T} . S}{2}$, the necessary criteria for Lyapunov stability of the observer was calculated as $0>g>-1$. In addition, the adequate criterion are calculated as $((1+g) / h)>\left(2\left|E_{a}-E_{b}\right| / a_{i} \cdot V_{d c}\right)$ and $((1+g) / h)>\left(2\left|E_{b}-E_{c}\right| / a_{i} \cdot V_{d c}\right)$. The adequate criteria imply that the hysteresis band should be enough small to make the left side of the equation larger than the right side. $0<a_{i} \leq 1$ was a reference speed-dependent parameter that adjust the fixed $V_{d c}$ at different speed bands. The properly chosen $a_{i}$ improve the dynamic response especially at low speeds. For very low speeds, $a_{i} \rightarrow 0$ and for high speeds $a_{i} \rightarrow 1$ improves the response of the system. In the physics-based observer, the equivalent resistance was adjusted at different winding temperature; though there may still a difference between the real and estimated temperature. An adjustment factor, 
$0.8<y<1.2$, can be multiplied to the equivalent resistance matrix to ensure us that the observer was always a bit faster than the actual machine. The improper choice of $y$ produce unwanted oscillations in the dynamic response of the system.

\subsubsection{Calculation of the parameters for physics-based observer}

As was shown in the motion equation of the observer, the accuracy and the convergence of the observer, depends of the following conditions and assumptions:

$$
\begin{aligned}
& V_{a b}=V_{a b}^{*} \& V_{b c}=V_{b c}^{*} \\
& A\left(\theta, i_{d}, i_{q}\right)=\hat{A}\left(\hat{\theta}, i_{d}, i_{q}\right) \\
& B(T)=\hat{B}(\hat{T}) \\
& 0>g>-1 \\
& ((1+g) / h)>\left(2\left|E_{b}-E_{c}\right| / a_{i} \cdot V_{d c}\right) \&((1+g) / h)>\left(2\left|E_{b}-E_{c}\right| / a_{i} \cdot V_{d c}\right)
\end{aligned}
$$

The respective necessary and adequate conditions (5-24-d) and (5-24-e) are achievable given the assumptions (5-24-a), (5-24-b) and (5-24-c) are satisfied. The $V_{a b}^{*}$ and $V_{b c}^{*}$ are accessible from inside the drive current control loop in a CSIbased VSI. Therefore the assumption (5-24-a) was achievable simply. The voltage $V_{a b}^{*} \quad$ and $V_{b c}^{*}$ must be retuned if the long length cable or line inductors are used between the drive and the PMSM and the drive hardware.

In order to satisfy the condition (5-24-b), accurate knowledge of the inductance and inductance variation with saturation is needed. Here, the voltage-based inductance calculation method explained in chapter 3 , was used to evaluate the instantaneous inductances as a function of the rotor position and saturation. Fortunately, this technique enables us to evaluate the inductance at different rotor positions and saturation levels. This technique accounts for the effect of magnets on the saturation - 


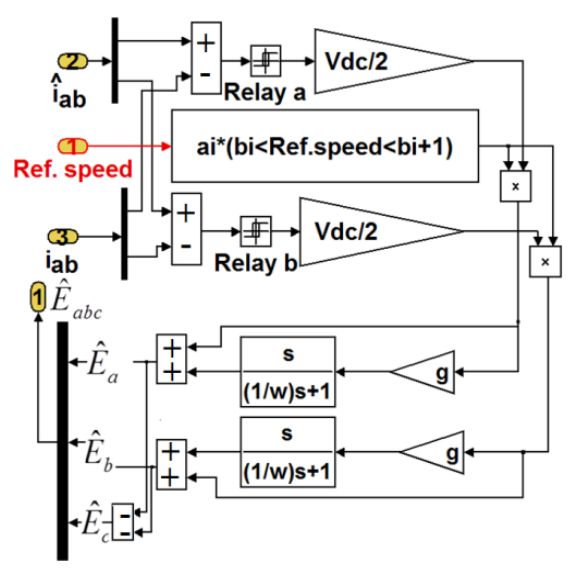

Figure 5.1 Hysteresis EMF estimator subsystem

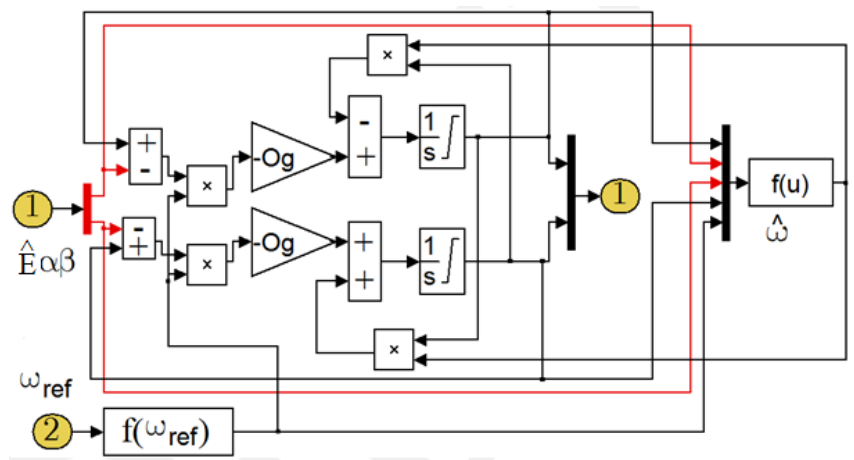

Figure 5.2 Adaptive angle compensator subsystem

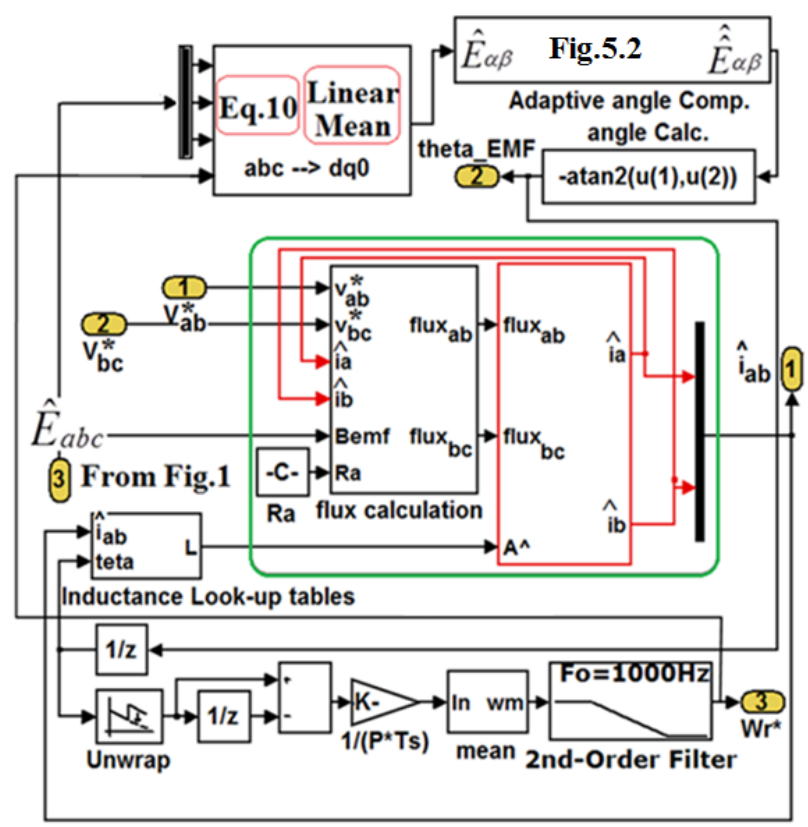

Figure 5.3 Combination of Physics-based PM circuit model, adaptive angle compensator, and hysteresis estimator subsystems 
of the machine. The respective inductance of each phase for different current snapshots was then saved in multidimensional array, 3-D lookup tables. Simply, the linear interpolation can be used to interpolate the value of inductance at different rotor positions and snapshots of the current break points. In order to reduce the number of field computations required by the inductance determination method, the inductances can be chosen as a function of $i_{d}$ and $i_{q}$ instead of $i_{a b c}$. This is useful especially in the case of vector control which is used in drive control loops. Moreover, in order to have an exact symmetrical inductance matrix, i.e. $L_{a b}=L_{b a}$, the average value, $L_{a b}^{a v}=\left(L_{a b}+L_{b a} / 2\right)$, is assumed for both. The inductance look up Tables are all independently excited by the instantaneous value of each phase current and by the rotor position which was calculated from sensorless algorithm, see figure 5.3. It was mentioned that in the FE domain the initial rotor position used for inductance calculation must be added to estimated rotor position and then it was applied on the look-up Tables.

Open loop observers suffer from sensitivity to system noise, changes in the stator resistance and changes in Magnet's Residual induction $\left(B_{m r}\right)$. The changes in $B_{m r}$ due to normal or over temperature strongly depend on magnet specific temperature data and magnet geometry. In general, the rate of change of the stator resistance per degree Celsius was much greater than the rate of change of the $B_{m r}$ in rare earth magnets and therefore the latter can be ignored. Although the thermal time constant of the machine was much lower than the electrical and the mechanical parts, during a long term operation the stator resistance can increase with up to $50 \%$ due to heat. The problem with changing resistance becomes evident at low speed as the voltage drop 
over the stator resistance then is of the same magnitude as the EMF. As a result, the need for a lumped-parameter transient thermal model for SMPMSM synchronous machine was necessary. In order to satisfy the condition (5-24-c), the estimation of the winding temperature was required. It was developed by using an embedded thermal network of the machine. The evaluation of the thermal network was explained earlier in chapter 3. Although, the methods used for calculation of the winding temperature was accurate enough to satisfy the assumption made for convergence of the observer, the tuning factor $0.8<y<1.2$, was used for final tuning of the observer in real-time environment.

\subsection{Case of study}

A $250[\mathrm{~W}]$, 10-pole, $42[\mathrm{~V}]$ surface mount permanent magnet synchronous machine (SMPMSM) is tested. The rated speed is $420(\mathrm{rad} / \mathrm{s})$, the resistance/phase at $20^{\circ}(C)$ is $0.0625(\Omega)$, the stator core material is M19, The rotor is steel 1018 , the shaft was stainless steel and the magnets are uniform Sm2Co17, with remnant flux density of magnet $B_{m r}$ at $20^{\circ}(C)$ equals to 1.07 Tesla. The magnet's intrinsic coercivity, $H_{c i}$ equals to $26(\mathrm{kOe})$ or $2.1(\mathrm{kA} / \mathrm{m})$. The complete machine geometry as well as the winding arrangement was illustrated in figure 5.4.

\subsection{Simulation and experimental results}

Three ranges of speed are tested i.e. medium low speed in that $10<\mid$ Reference speed $(\mathrm{rad} / \mathrm{s}) \mid<20$, low speed in that $2<\mid$ Reference speed $(\mathrm{rad} / \mathrm{s}) \mid<10$ and very low speed in that $0.5<\mid$ reference speed $(\mathrm{rad} / \mathrm{s}) \mid<2$. For adjustment of the band width of the speed PI controller for wide speed range, the proportional gain of the speed PI controller varies with speed in such a way that in high/low speeds, lower/higher proportional gains was multiplied with the speed error. The Simulink set up of the 
sensorless schema are shown in Figures 5.5. The depicted self and mutual inductances in figures 5.6 and 5.7 at different saturation level were calculated from a transient FE analysis with motion. The saturation level refers to $I_{i}=\left[i_{a}, i_{b}, i_{c}\right]=\left[K_{i},-0.5 K_{i},-0.5 K_{i}\right]$ for $K_{i}=1,2,4,8,16$. The flux linkage and the cogging torque of the machine are shown in figure 5.8.

Figures 5.9 to 5.16 shows the simulation and experimental results for speed, rotor position, phase currents, EMF, operational inductances and estimation error for the constant load (20\% of nominal) and step changes in reference speed inside the boundary of $2<\mid$ Reference speed $(\mathrm{rad} / \mathrm{s}) \mid<13$. In these figures, the thickness of the measurement solid line has $10 \%$ error, side to side. In percent, the studied boundary of speed was $0.1(\%)<\mid$ reference speed/rated speed $\mid<3.11(\%)$. It was seen that, the lower the speed, the higher current THD and the estimation error will be. Moreover, these results prove the controllability of the speed as well as the high accuracy of estimated rotor position for medium low and low speeds.

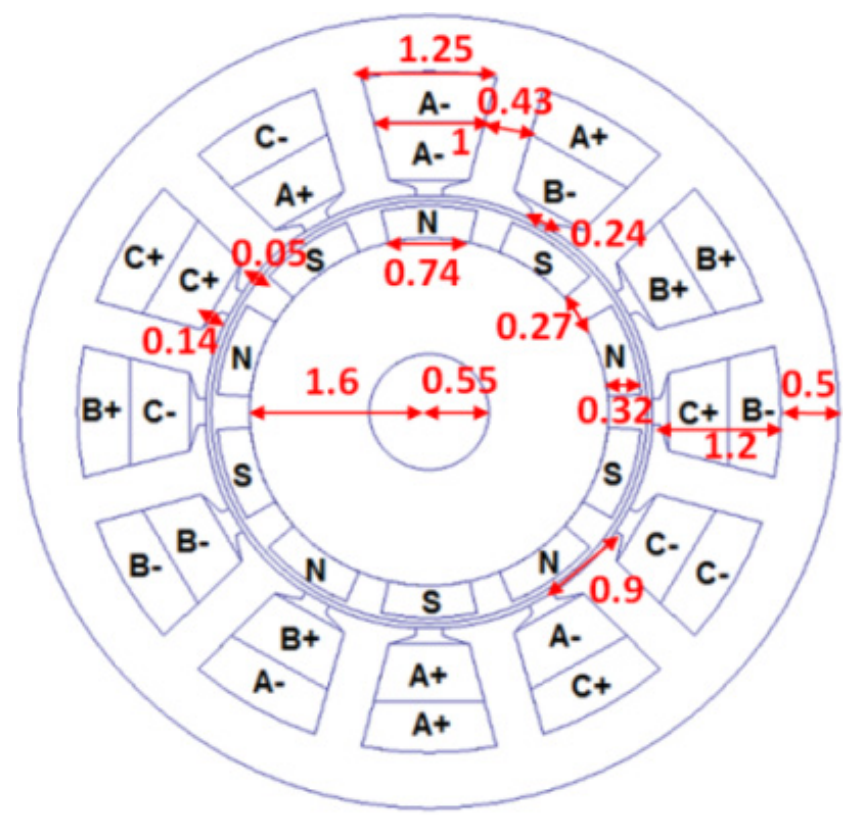

Figure 5.4 Geometry $(\mathrm{Cm})$ and winding arrangement of the studied machine 


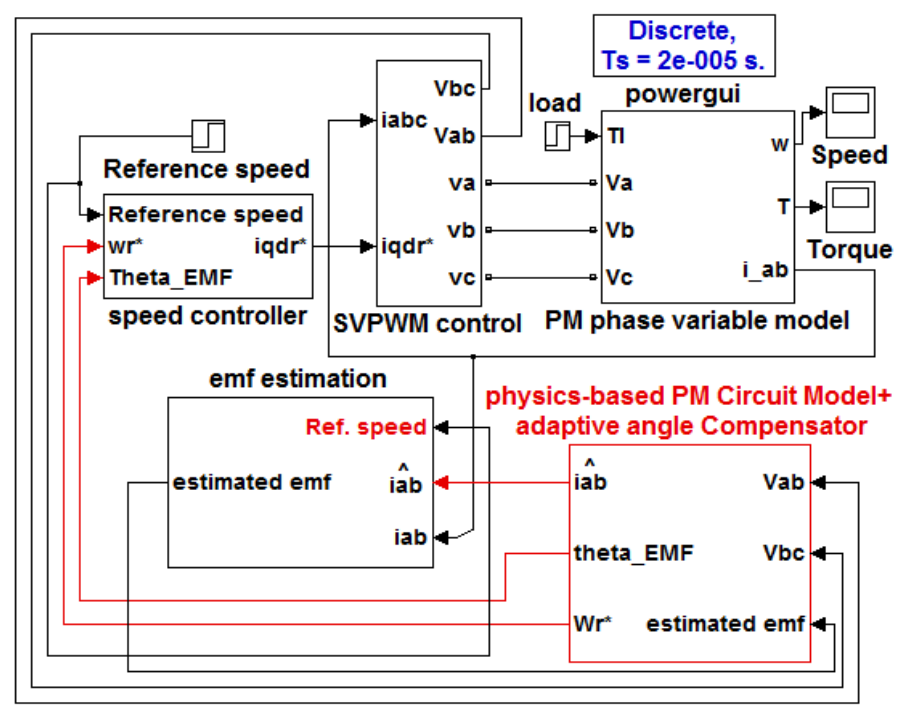

Figure 5.5 Sensorless speed control in Simulink domain

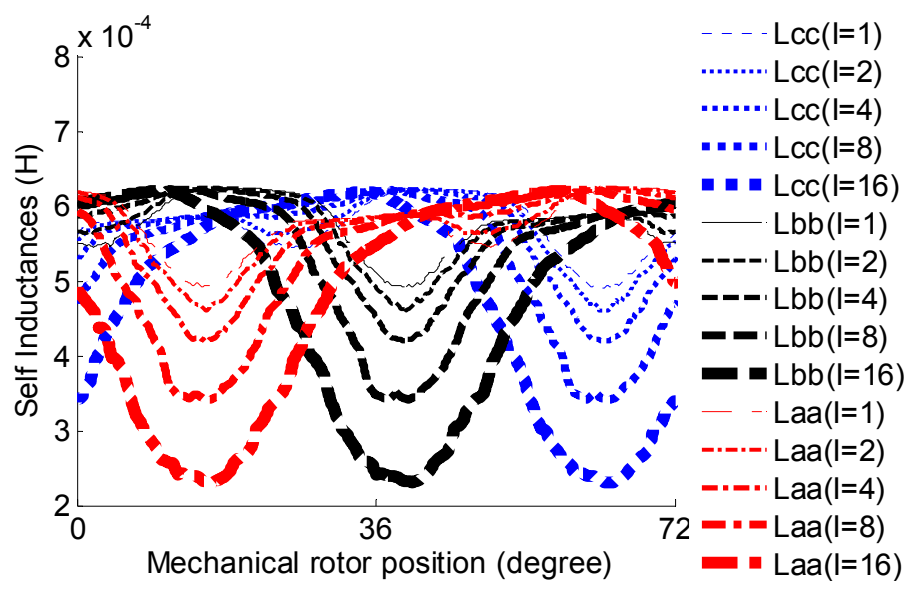

Figure 5.6 Calculated self-inductance at five saturation level

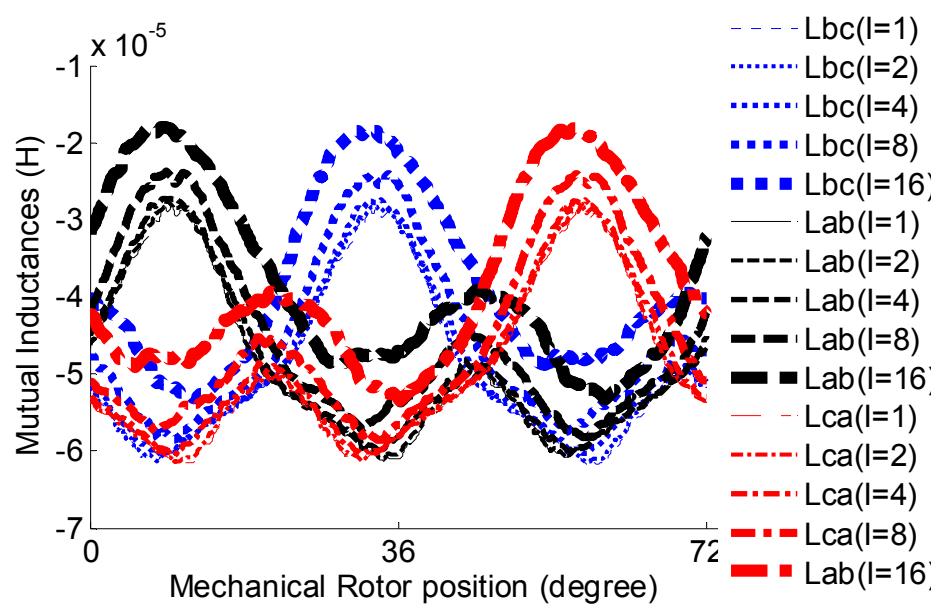

Figure 5.7 Calculated mutual inductance at five saturation level 


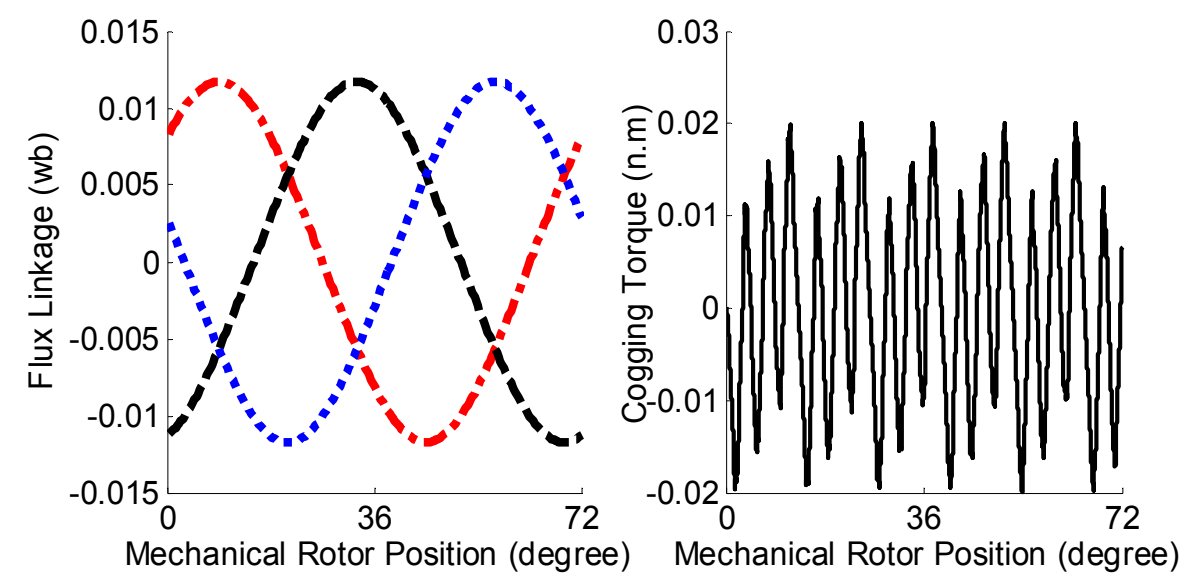

Figure 5.8 Flux linkage and cogging torque of the studied machine

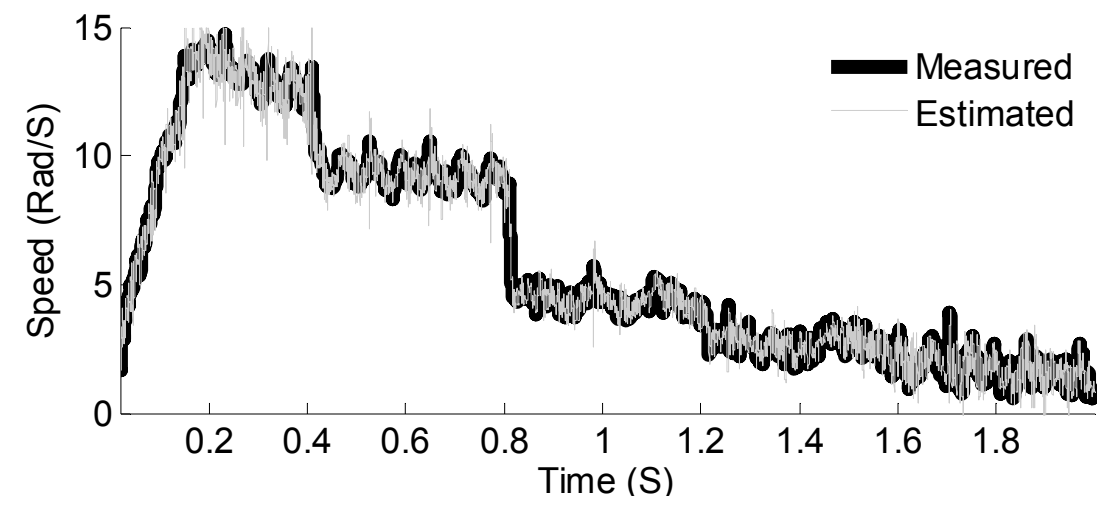

Figure 5.9 Estimated versus measured speed changes due to Step change in reference speed for $20 \%$ nominal load (simulation)

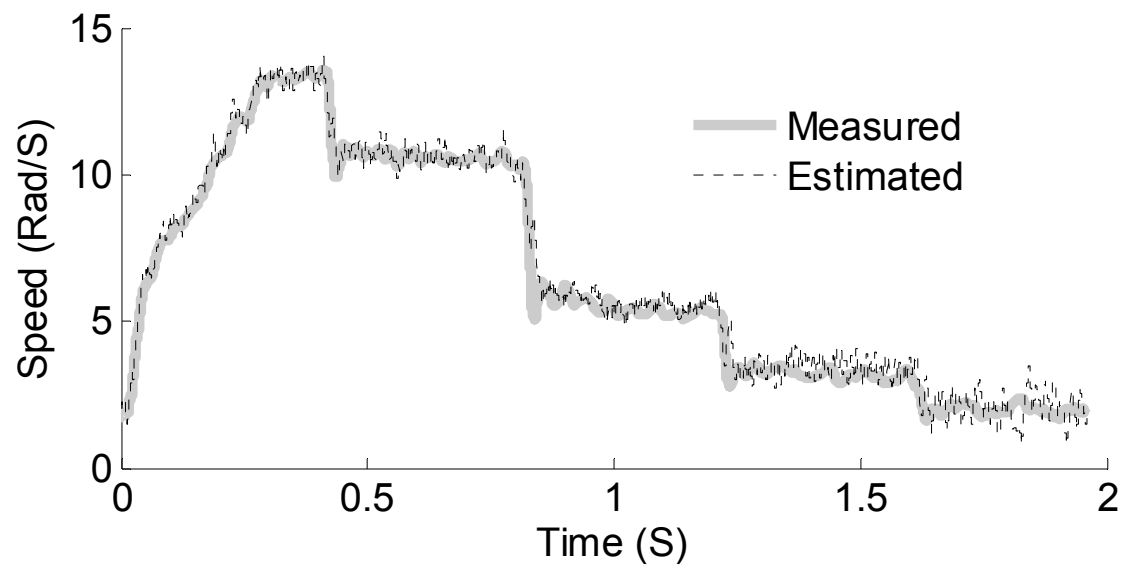

Figure 5.10 Estimated versus measured speed changes due to Step change in reference speed for $20 \%$ nominal load (experimental) 


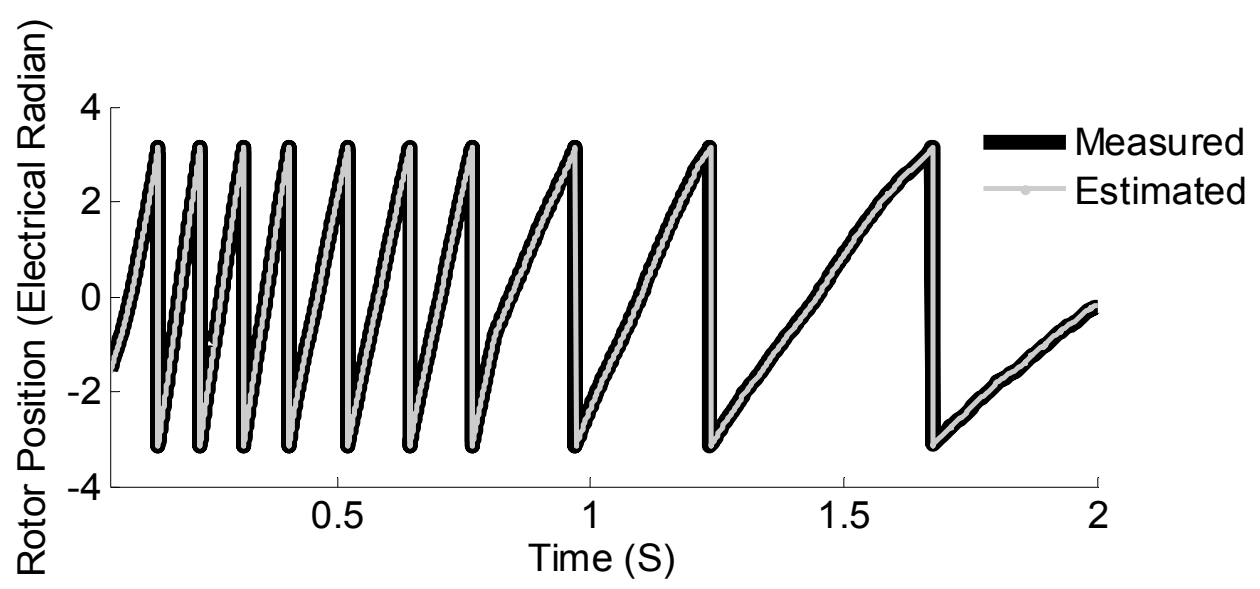

Figure 5.11 Rotor position changes due to Step change in reference speed for $20 \%$ nominal load (Simulation)

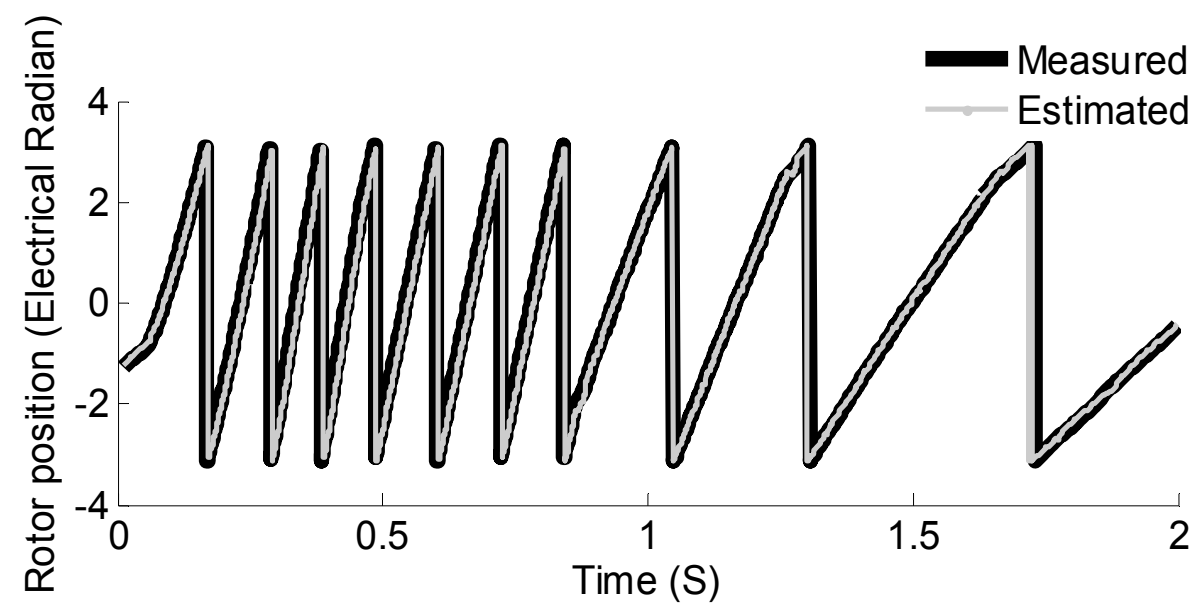

Figure 5.12 Rotor position changes due to Step change in reference speed at $20 \%$ nominal load (Experimental)

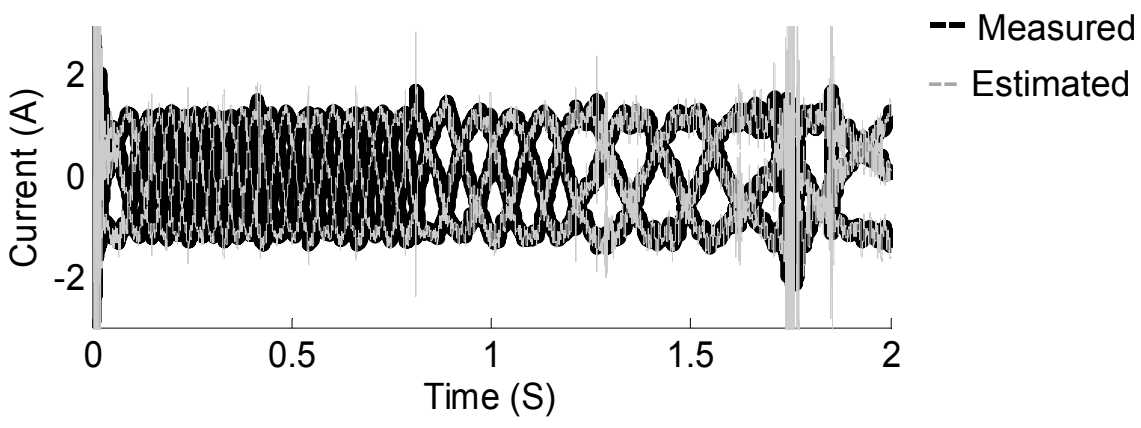

Figure 5.13 Phase current changes due to Step change in reference speed under $20 \%$ nominal load (Experimental) 


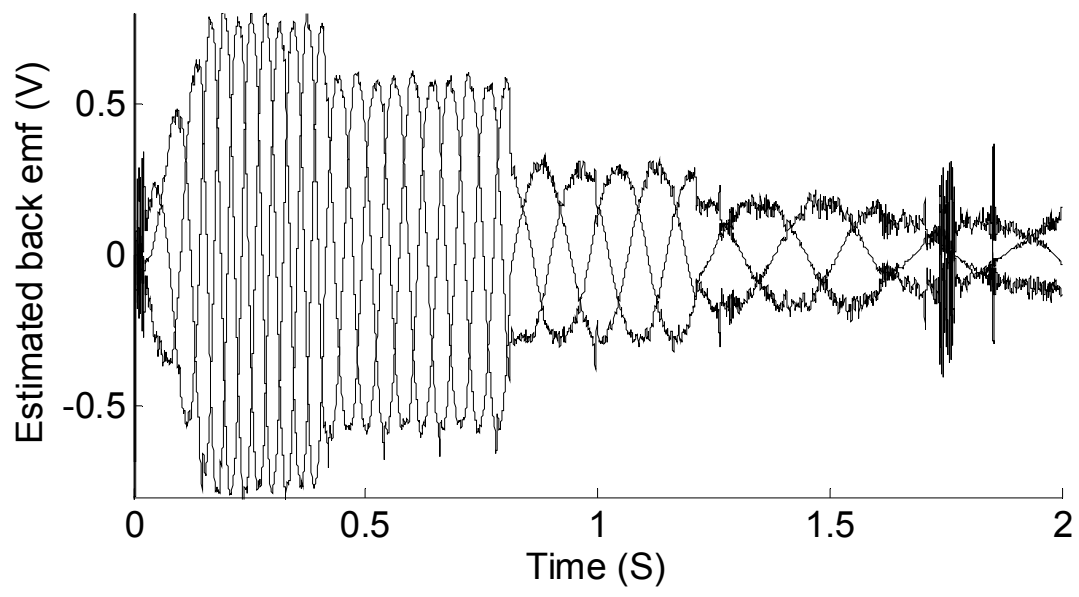

Figure 5.14 EMF changes due to Step change in reference speed under $20 \%$ nominal load (Experimental)

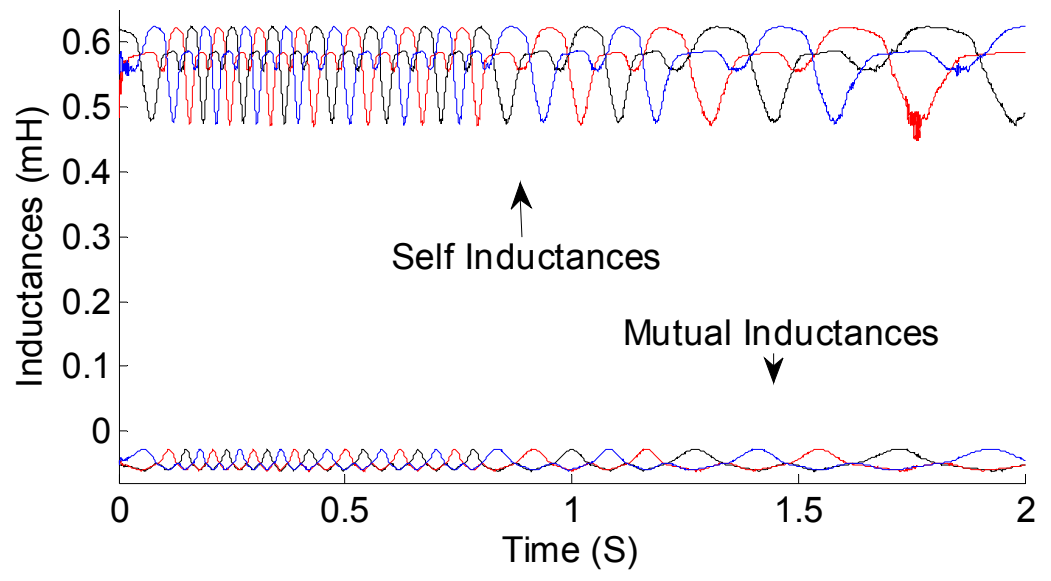

Figure 5.15 Operational inductance due to Step change in reference speed under $20 \%$ nominal load (Experimental)

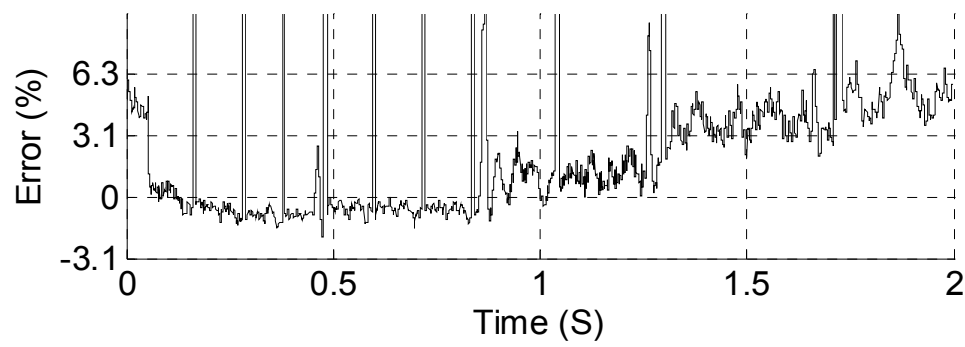

Figure 5.16 Presented error due to Step change in reference speed under $20 \%$ nominal load (Experimental)

Figure 5.17 shows the experimental results of the estimated versus measured rotor 
positions for very low speed, $0.5(\mathrm{rad} / \mathrm{s})$ i.e. 0.1 percent of the rated speed. During this experiment it was seen that with a fixed adaptive angle, the compensator constants, $A_{l}$ and $O_{g}$, the lowest possible speed by this method was $0.5(\mathrm{rad} / \mathrm{s})$ in which the loadability decreases to $10 \%$ of the nominal load. One of the main problems in the very low speed applications is seen in figure 5.18. In this figure, the estimated EMF was polluted by an incredible level of noise. Indeed by choosing the larger and smaller values for $A_{l}\left(\omega_{\text {ref }}\right)$ and $O_{g}\left(\omega_{\text {ref }}\right)$, the noise cancellation will be improved and the load-ability will be increased to nominal value. The larger and smaller values for $A_{l}\left(\omega_{\text {ref }}\right)$ and $O_{g}\left(\omega_{\text {ref }}\right)$ will slow down the rotor position estimation at medium and higher speeds, therefore adjustment of these two parameters for different reference speed changes was necessary. The lower/higher speeds, the larger/smaller and smaller/larger values for $A_{l}\left(\omega_{\text {ref }}\right)$ and $O_{g}\left(\omega_{\text {ref }}\right)$ was needed. Figure 5.19 illustrate the presented error with respect to figure 5.19. The maximum error was under six percent.

Figure 5.20 shows the validation of the introduced technique for the step load changes from $20 \%$ to $100 \%$ of nominal load. Figure 5.21 and figure 5.22 show the respective inductance and current for this operating condition. From figures 5.21 and 5.23 , the respective change of the inductance profile and the current was visible. In another study, the sudden voltage interruption to zero for a short period of time for the medium speed was studied. This was created by blocking the command signal to the inverter switches for $1(\mathrm{~ms})$. The results of this test appear in figure 5.23 on top of the same operating condition but without fault. It can be observed that firstly the rotor position estimation never loses and secondly after eight electrical cycles the speed of the faulted system matches with the normal system. In addition, the transient behavior of the estimation system was shown in figure 5.24. The estimation system could 
follow the measured rotor position after $3.5(\mathrm{~ms})$. Here, the sample time of the estimator was $0.02(\mathrm{~ms})$ and the hysteresis band was 0.001 . In the speed controller, the sample time $0.1(\mathrm{~ms})$; PWM switching frequency=25 $(\mathrm{kHz})$; the dead-time between the upper and the lower switches in 3-phase AC drive was $1(\mu s)$. Here, the number of data in look-up tables for one electrical cycle was chosen as 25000 . In addition, the full healthy thermal model was used. However, the number of data in the look-up tables as well as the thermal model using thevenin theorem could be reduced. In this way achieving to lower calculation time was possible.

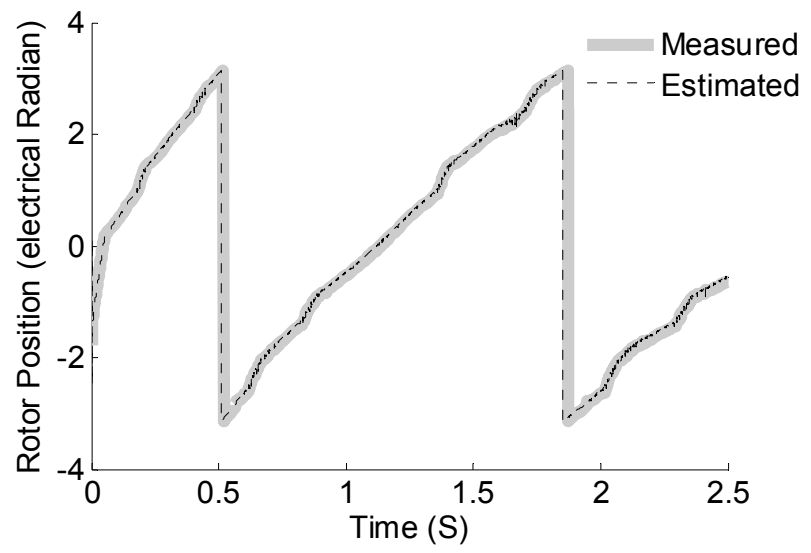

Figure 5.17 Rotor position for reference speed of $0.5(\mathrm{rad} / \mathrm{s})$ under nominal load (Experimental)

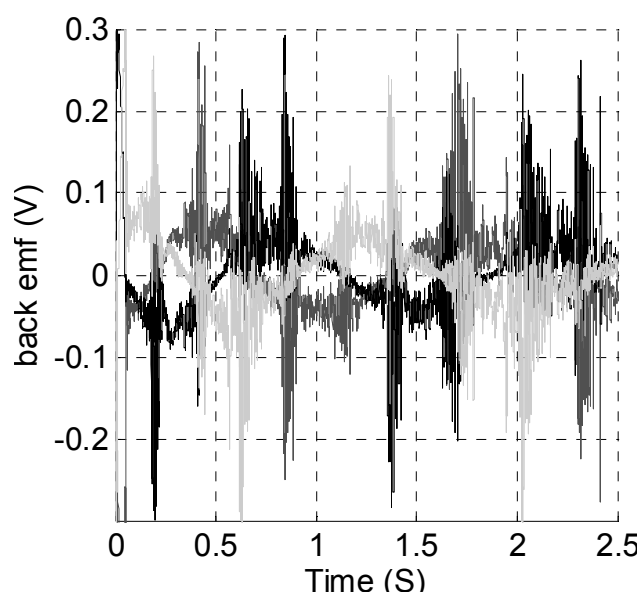

Figure 5.18 Estimated EMF for reference speed of $0.5(\mathrm{rad} / \mathrm{s})$ under nominal load (Experimental) 


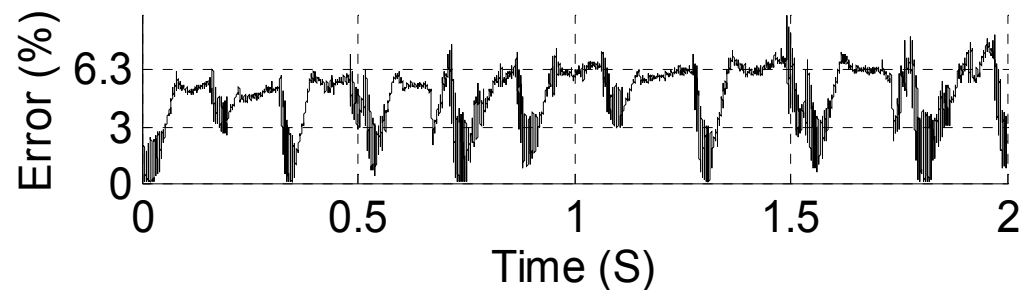

Figure 5.19 Presented error $(\%)$ for the reference speed of $0.5(\mathrm{rad} / \mathrm{s})$ under nominal load (Experimental)

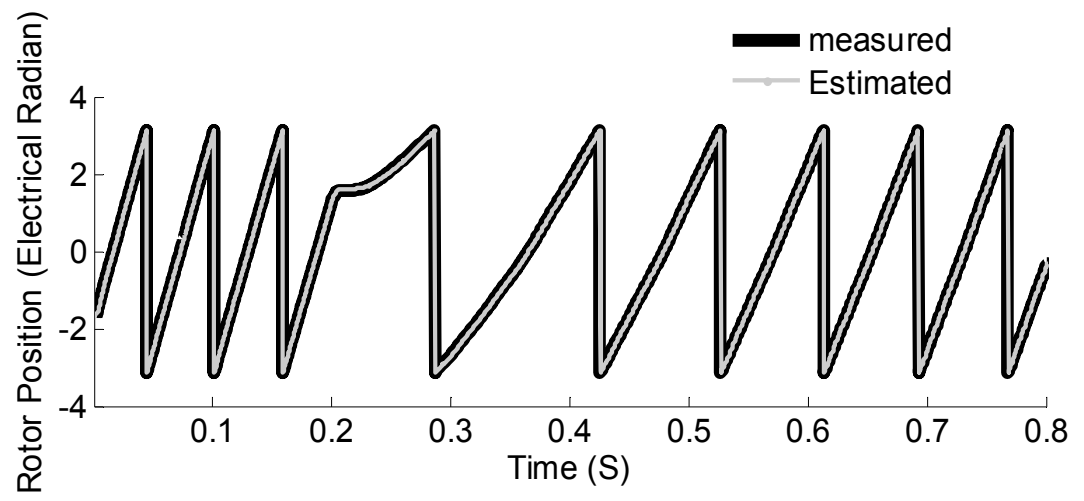

Figure 5.20 Rotor position changes due to Step change in load from $20 \%$ to $100 \%$ of nominal load at 0.2 (s) for reference speed of $20(\mathrm{rad} / \mathrm{s})$ (Experimental)

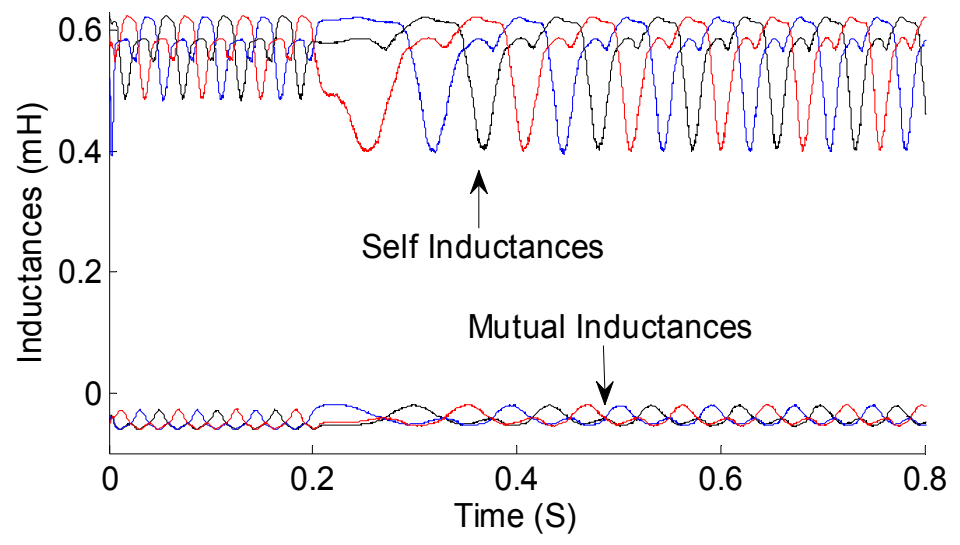

Figure 5.21 Operational inductance profile for Step change in load from $20 \%$ to $100 \%$ of nominal load at 0.2 (s) for reference speed of $20(\mathrm{rad} / \mathrm{s})$ (Experimental)

Figures 5.25 show the response of the sensorless control for the very slow reference speed reversal from $3(\mathrm{rad} / \mathrm{s})$ to $-3(\mathrm{rad} / \mathrm{s})$. Figure 5.26 show the respective estimation error of figure 5.25. Figures 5.27 show the response of the sensorless 
control for the very fast (step) reference speed reversal from $-3(\mathrm{rad} / \mathrm{s})$ to $3(\mathrm{rad} / \mathrm{s})$.

Figure 5.28 show the respective estimation error of figure 5.27. In figures 5.25 and 5.27 , the thickness of the measurement line has 10 percent error side to side.

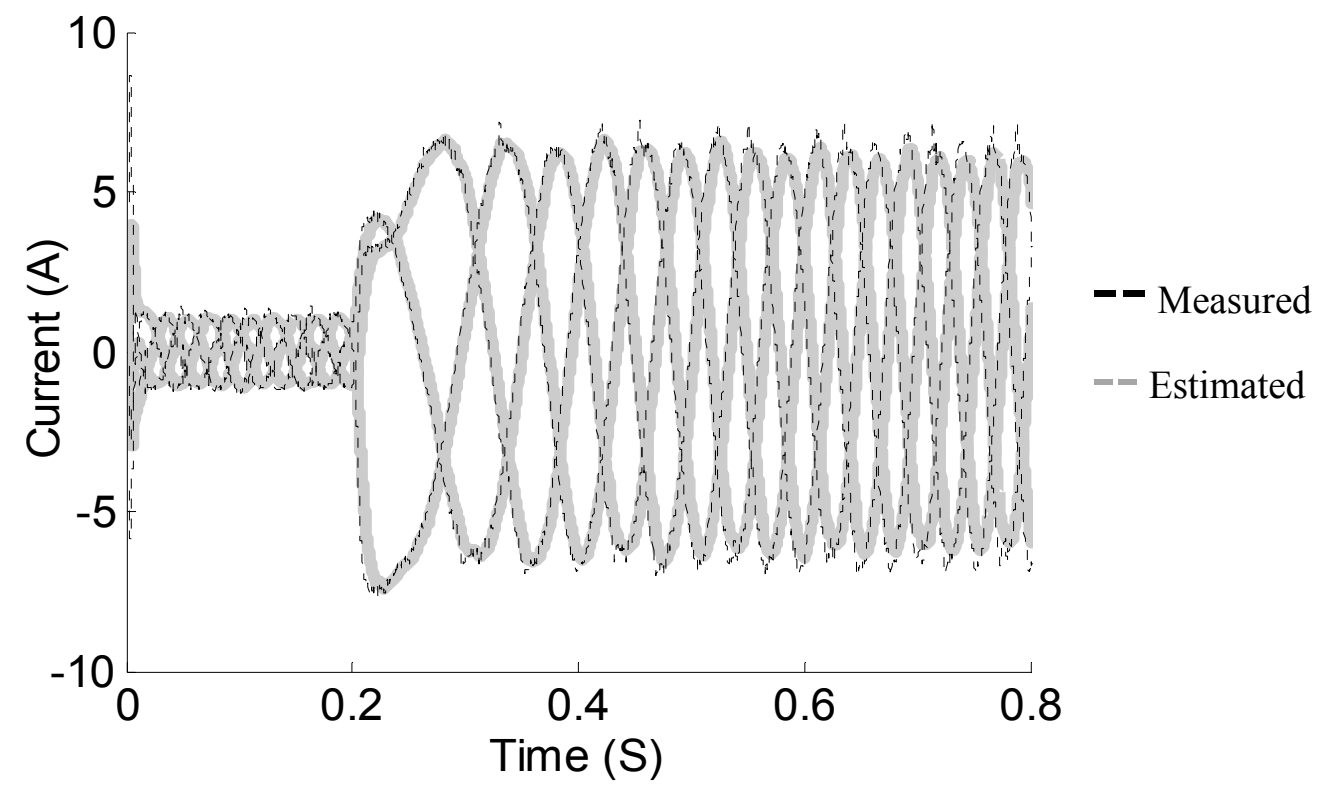

Figure 5.22 Current changes due to Step change in load from $20 \%$ to $100 \%$ of nominal load at 0.2 (s) for reference speed of $20(\mathrm{rad} / \mathrm{s})$ (Experimental)

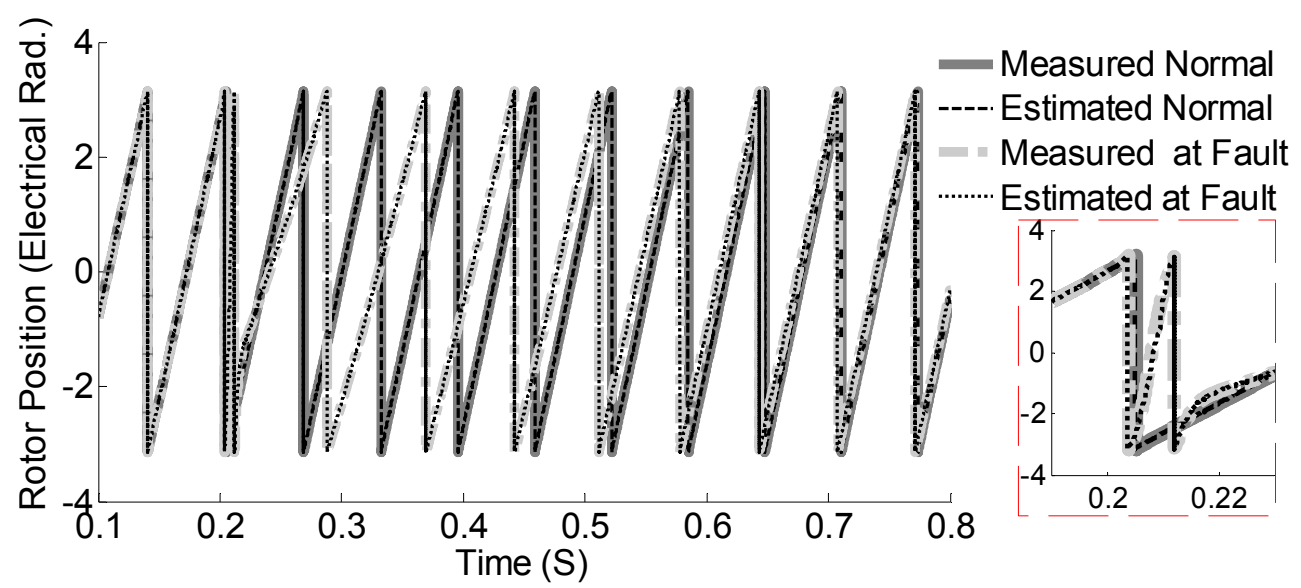

Figure 5.23 Operation of the sensorless operation under $20 \%$ nominal load under sudden voltage interruption to zero at $0.2<\mathrm{t}<0.21$ for speed of 20 $(\mathrm{rad} / \mathrm{s})$ 


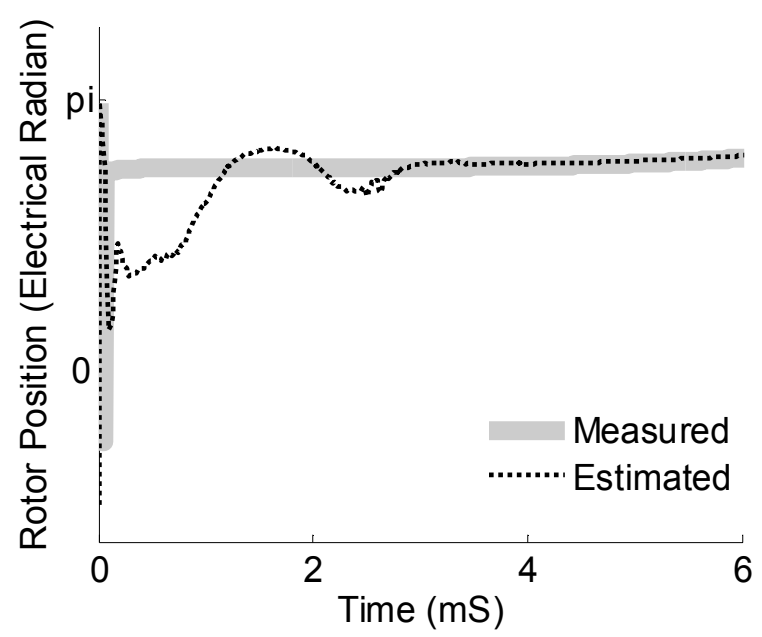

Figure 5.24 Initial transient during rotor position estimation

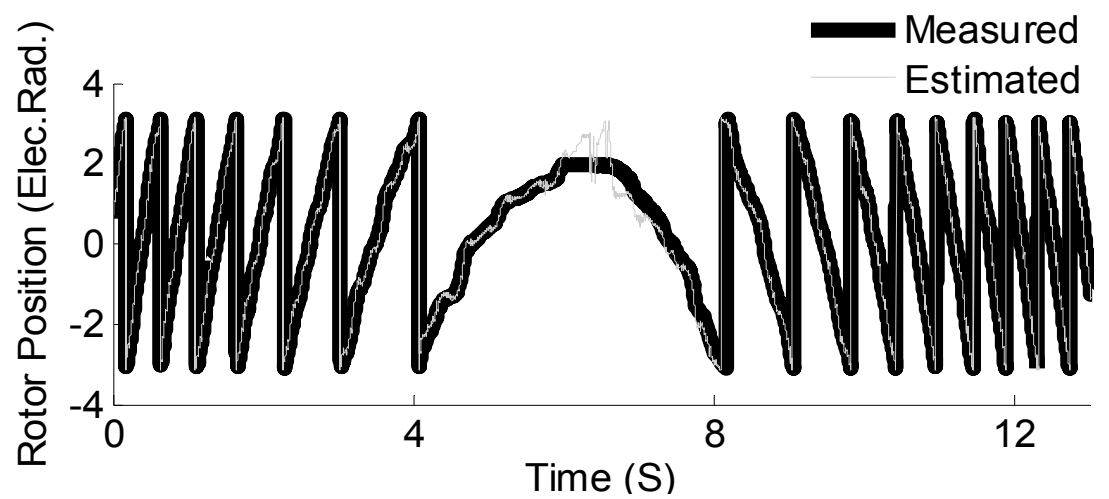

Figure 5.25 Rotor position for very slow reference speed reversal from 3 to -3 $(\mathrm{rad} / \mathrm{s})$ under nominal load (Experimental)

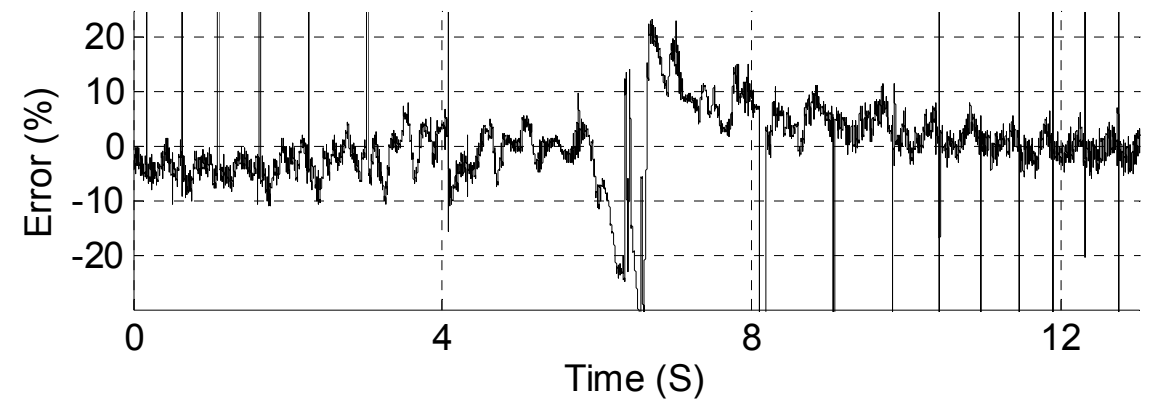

Figure 5.26 presented error due to very slow reference speed reversal from 3 to -3 $(\mathrm{rad} / \mathrm{s})$ under nominal load (Experimental) 


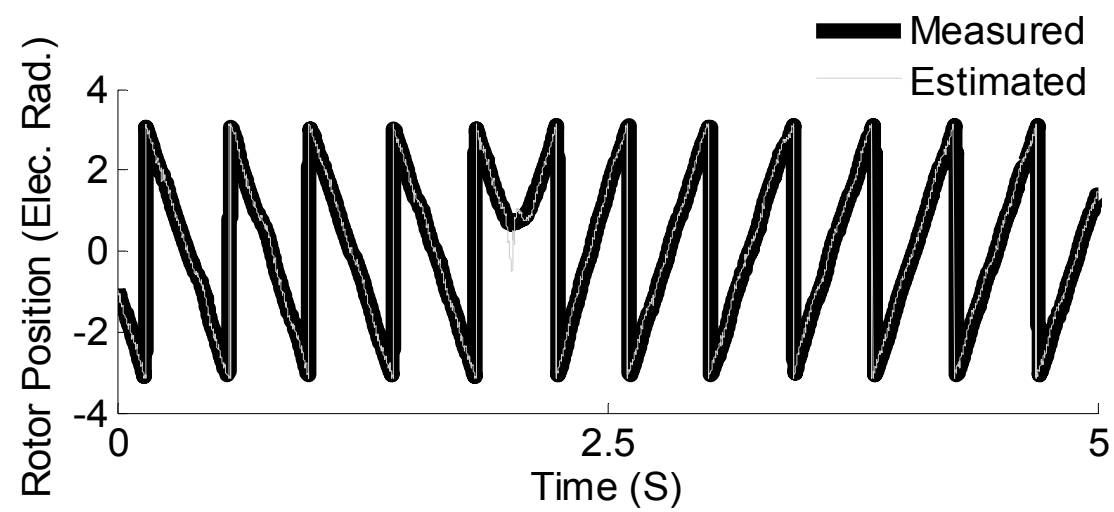

Figure 5.27 Rotor position for fast reference speed reversal from -3 to $3(\mathrm{rad} / \mathrm{s})$ under nominal load (Experimental)

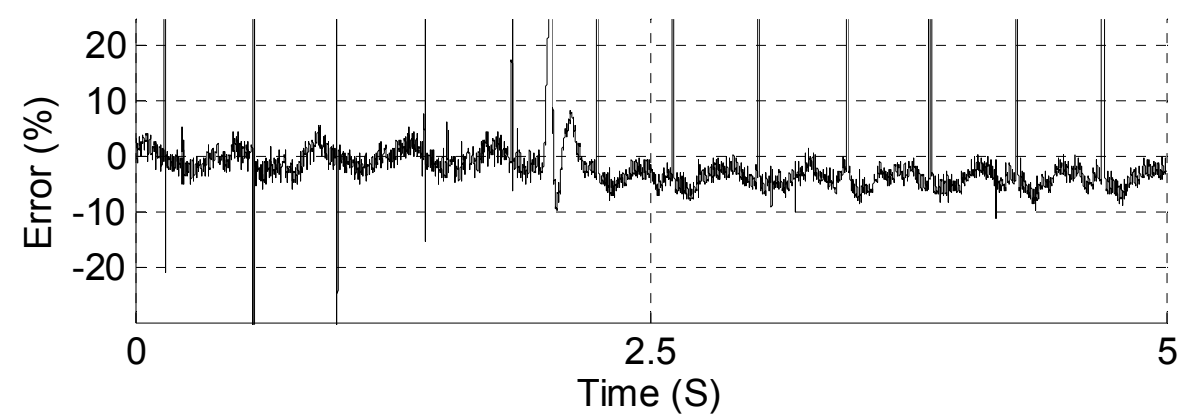

Figure 5.28 Presented error due to fast reference speed reversal from -3 to 3 $(\mathrm{rad} / \mathrm{s})$ under nominal load (Experimental)

\subsection{Summary}

A position sensorless control using a physics-based observer was developed. The will increase the applicability of the estimation based EMF observers for mediumlow, low and very low speeds. A novel thermoelectric physics-based modeling approach was developed to evaluate more realistic information about the instantaneous behavior of the machine during operation. For removing high frequency noise during angle calculations without missing the observer band width an adaptive angle compensator was designed. The simulation and experimental results under different speed, loading and voltage variation show acceptable accuracy. The proposed physics-based sensorless drive provides a rotor-position estimate, which was 
safe from system noise. It can also estimate the precise speed, because it can follow the machine model variations. The temperature was estimated using an embedded transient thermal model. The introduced sensorless control eliminates the need for simultaneous observation of the stator resistance, stator inductance and the rotor flux magnitude. 


\section{Chapter 6 Inter-turn Fault Detection in PM Synchronous Machines by Physics-based EMF Estimation}

\subsection{Overview}

In this chapter, the inter-turn short circuit fault detection in permanent magnet synchronous machines using an open loop physics-based back electromotive force estimator was presented. The back electromotive force estimator was designed based upon a current mode tracking scheme. The thermal and saturation aspects of the machine were considered in the design of the estimator. The design procedure and stability criteria of the estimator are presented here in detail. The fault detection was carried out based on the difference between the estimated back electromotive force and a reference back electromotive force. A $0.8(\mathrm{~kW})$ permanent magnet synchronous machine was studied experimentally and numerically studied under different interturn fault and operational contingencies. The numerical modeling was accomplished by a finite element-based model coupled with the thermal network and polluted with inter-turn fault. The acceptable agreement between the simulated and experimental result validates the modeling process. The back electromotive force estimator fault detection system has led to discriminative inter-turn fault signatures in a fraction of second for wide speed range even in the presence of harmonic loads and dynamic eccentricities.

\subsection{Inter-turn fault diagnosis in PMSM}

The progressive behavior of inter-turn short circuit fault (ISCF) [171] and the irreversible demagnetization of permanent magnet $(\mathrm{PM})$ are the main reasons that forces to shut down or immediately start the critical remedial actions in permanent magnet synchronous machine (PMSM) if the fault was detected and diagnosed as 
inter-turn short circuit. The intensity of ISCF in a PMSM changes with the change in the PMSM design parameters. For example, the comparison between two PMSM with distributed and the concentrated windings show that the typical copper slot fill factor in distributed windings was about $34 \%$ to $45 \%$ where in the concentrated windings it was around $50 \%$ to $65 \%$. The slot fill factor in concentrated windings was higher particularly when the segmented stator structure was used. This means that the distance between the neighbor conductors in a slot was lower in concentrated windings and therefore one can conclude that inter-turn fault growth rate in the concentrated windings may be higher than that of the distributed windings. However, the non-overlapping and concentrated windings, particularly the single layer windings, provide many advantages in terms of fault tolerance. Single layer windings provide very low mutual coupling between the various phases. The winding's coils are physically separated and therefore the shorted turns contact was limited to faulty coil[100]. Other design parameters of a PMSM such as turn resistance, flux linkage per turn and saturation level of the machine also affect the intensity of the inter-turn fault [172]. On the other hand, the intensity of ISCF was also related to the PMSM speed, the ambient temperature and drive control loop. For example, because of high ambient temperature a few ISCF may lead to catastrophic damage in a few seconds while in another situation because of low ambient temperature and higher heat transfer rate even several shorted turns might not lead to quick insulation deterioration.

Vibration and thermal analysis has been proven as reliable methods in multi-fault diagnosis of induction machines [173]. Such approaches can be extended for PMSM. However, consideration of the cost of extra sensors and additional hardware interfaces in the overall cost of fault detection systems was a serious objective. Hence, the usage 
of already available signals from the machine drive systems has been extensively encouraged in many investigations [174].

Although the investigation in off-line periodic insulation inspection was well satisfied, the natural limitation on these methods has encouraged many investigations toward development of on-line turn fault detection methods [175]. Most of the techniques deliberate inter-turn fault studies in utility-driven motor applications and only a few turn fault detection methods for motor-drive applications [176]. Besides the research on a turn fault detection method for close loop PMSM-drive applications still remains unfulfilled.

The motor-drive systems necessitate compound analysis to address problems such as high noise/signal ratio, non-stationary behavior of fault signatures, dependability of fault signature frequencies to operating points and more importantly the closed-loop control effects, etc.[177]. Typically, the closed-loop motor-drive systems contain a slow outer low-bandwidth speed-regulating loop which generates the torque command for the fast inner high-bandwidth current-regulating loop. Generally, the speed oscillation caused by the incipient turn fault was very small in magnitude and in almost all cases; the oscillating frequency was well above the bandwidth of the speed loop. The bandwidth of the fast inner current loop, however, was usually much higher than the frequency of the incidents that appears due to the inter-turn fault. Therefore, the actual current of the machine was forced to be exactly the same with the current command. This regulating effect of the current regulator can suppress any effect induced by the stator turn fault. This idea was employed in stator turn-fault detector and locator in multi-machine-drive systems[177]. However for the real-time implementation, the aforementioned approach suffers from issues such as obtaining the characteristics of the healthy motor-drive, compensation for dc voltage variation 
and accounting for various inverter non-ideal behaviors[177]. On the other hand, in variable speed drives the stator current and voltage frequencies locally or globally varies depending on the applied load and speed command. In fault conditions, the fault signatures shift in the current spectrum and make the fault signature tracking difficult. Even in steady state conditions the fault signatures in the current spectrum oscillates in presence of harmonic loads and/or speed oscillations[178]. In addition to this, the noise and various harmonic contents in the current spectrum due to harmonic loads and machine nonlinearities diminish the accuracy of most frequency analysesbased methods [179]. The time-frequency analysis has been introduced as an alternative solution to frequency-analysis methods because apparently the phase currents and modulated operating-point-dependent fault signatures are non-stationary signals. The time-frequency spectrum analysis was typically shown to be practical for commercial implementations. However, the complex time-frequency characteristics during the experimental stage, hardware limitations of low-cost real-time monitoring systems and the need for the base analytical frequencies and/or the base spectrums related to faults make these methods quite cumbersome in PMSM machines [180].

The model-based fault diagnosis systems have been emerged as a possible solution to end up the limitations of spectrum-based fault diagnosis systems in variable-speed drives. In model-based techniques, input to the machine was also fed to the model as well. Signals from both the real machine and the model are learned simultaneously and send to the fault decision making process [181].

In fault detection, the two important factors include the choice of proper signal and the decision making technique [181]. In recent literature [181-190], the extraction of fault features from different signals in both induction and PM machines was typically satisfied. However, redundancy in fault detection systems by adding search 
coil,[188], the need for additional measurement system[186], underestimation of fault and non-sinusoidal armature flux linkage harmonics as well as the adverse effect of unbalance in the supply voltage [185], underestimation of non-stationary behavior of fault on the reference voltage as well as the poor results during transients [183], poor result during transients[190], computational complexity[184], the tuning of the lowpass filters, nonlinearities in the reference system and inherit asymmetric of drive system[187], the need for large volume of training data[182], unsuitability for online application [189] are some of the limitations that exists in these methods.

In PMSM when an ISCF with zero resistance occurs, an unwanted current start circulating through the shorted turns to create a flux linkage out of phase in PM flux linkage. This causes a combination of minor local quadrature and major direct magnetization to appear in the iron. Depending on the ratio between the quadrature and direct demagnetization changes due to the fault and the inverter drive control loop, the inter-turn fault signatures are distributed between the current, voltage and electromagnetic torque. The degree of this distribution depends on the bandwidth of the outer speed and inner current loop PI controller, the operating point of the machine. Therefore the combined usage of current, voltage and speed in form of an observer scheme were proposed in [191-193]. In[193] the EMF estimation was used for a PMSM-drive system. However, the lack of accuracy on the estimator parameter predictions, underestimation of the thermal effects and the load variations has limited the method to just steady state operation of the machine and also the method fails to detect the emerging faults [192].

In order to design a sensitive model-based EMF estimation system for a 3-phase PMSM, a computationally light but accurate model that can handle the nonlinearities, thermal aspects and unbalancing conditions of the PMSM was needed. Analytical 
modeling methods, although broadly used, are not precise. The FE-based models alternatively can be classified into four major categories i.e. full finite element(FE) models, data-based models like FE-based d-q models, the FE-based phase variable model, the field reconstruction method (FRM), and reluctance network based models[181].

The finite element in a coupled field-circuit analysis domain offers the most accurate modeling methods but it was extremely heavy for real-time applications. A simple FE-based d-q model was computationally very light but it was not useful enough for fault diagnosis purposes under a phase unbalancing condition. The FRM was used to estimate the flux linkage in each stator slot. The reluctance network models and the FRM methods are computationally less intensive compared to the full FE model because only one stator slot was modeled. Also the numerical modeling of the reluctance network model requires less computational burden than the FE models. Nevertheless, these are still computationally heavier methods than data-based models. The FE-based phase variable model show good computational time. This FE-based phase variable model was a data-based model which has shown to be suitable for fault diagnosis studies as well as modeling of machine-drive systems[181], [188].

After measurement or estimation of fault signal, the two major decision making techniques include the hard threshold-based techniques and the knowledge-based techniques. The measured fault signal can directly or indirectly after signal processing or feature extraction can be employed in these methods. The knowledge based systems are more accurate but the threshold-based systems are computationally more desirable [181].

In this chapter, the employed signal for fault detection was the phase side back electromotive force (EMF). The decision making technique was a threshold-based 
one. The fault index was average differences between the real time estimated and a reference EMF. The reference EMF was initially calculated either by an FE analysis, or by real measurement at a known speed from the healthy machine, or by estimation of EMF from healthy machine. The latter was preferable because it bypass all the uncertainties that are not included in the estimator design or calibration stage. The reference EMF was collected in three 1-D look up Table. The look up Table was controlled by speed and rotor position. The EMF estimation system covers all the nonlinearities, thermal aspect of the machine and therefore as long as there was no fault inside the machine the real machine EMF and the estimated EMF are very close. This prevents wrong fault alarm. However, when the inter-turn fault was occurred inside the real machine because of the difference between the machine model employed in EMF estimator and the actual machine, the estimated EMF differ from the reference EMF. The complete description of the reasons and the physical meaning of these differences are presented in section (V).

In this chapter, the software simulation was assisted in prediction of what that probably happens to the actual machine under an ISCF. This helps choosing the proper test set up in order to prevent potential damage to the actual PMSM during experimental ISCF studies. The simulation and experimental results are compared.

\subsection{Inter-turn fault modeling for 3-phase PMSM without access to neutral point}

The Inter-turn faults increase the slot leakage flux and cause iron saturation. Under inter-turn fault, the inductances are no longer functions of the number of turns and slot geometry only. However, they are also dependent on the material property and the location of the faulted turns in the slot. Here, for initial fault studies a FEbased model was used. The creation of this model consists of two general steps. In the first step, a non-linear transient FE analysis was performed to calculate the cogging 
torque, EMF, PM flux linkage and the inductance matrix of the machine. The employed equations for calculation of these parameters presented in earlier in chapter 3 sections 3.4 to 3.10 . In the next step, these calculated parameters are collected in look up tables to be employed in the machine voltage equations (3-180) and the motion equations (3-181). For further details, the reader should consult chapter 3 section 3.21.4. The utilized technique for inductance calculation is an FE-based technique which was presented in chapter 3 section 3.10 . The inductances were calculated as a function of rotor position, $\theta$ and the saturation levels $\left(i_{d}, i_{q}\right)$. The resultant flux from positive $i_{d}$ and $i_{f}$ are set to be exactly in opposite with PM flux linkage. The resultant flux from $i_{q}$ was set to be perpendicular with the PM flux linkage.

\subsection{Design of EMF estimation system for inter-turn fault detection}

The EMF estimator was designed based on a stator current tracking scheme. Due to the fact that, the stator currents are directly measurable in a PMSM drive, the tracking path was selected on the real stator current trajectory. When the difference between the actual the estimated currents become nonzero, a PI controller compensates the EMF in a very short period of time. This is to force the current estimation error to become zero. In a healthy PM machine without access to neutral the line-line voltage formulation can be used for design of estimator as:

$$
\begin{aligned}
& {\left[\begin{array}{l}
\hat{i}_{a}(t) \\
\hat{i}_{b}(t)
\end{array}\right]=\hat{A}\left(\theta(\tau), i_{d}(\tau), i_{q}(\tau)\right)^{-1} \times} \\
& \qquad \int_{0}^{t}\left\{\hat{B}(T(\tau)) \cdot\left[\begin{array}{c}
\hat{i}_{a}(\tau) \\
\hat{i}_{b}(\tau)
\end{array}\right]+\left[\begin{array}{c}
V_{a b}^{*}(\tau) \\
V_{b c}^{*}(\tau)
\end{array}\right]+\left[\begin{array}{c}
\hat{E}_{b}(\hat{\theta}(\tau))-\hat{E}_{a}(\hat{\theta}(\tau)) \\
\hat{E}_{c}(\hat{\theta}(\tau))-\hat{E}_{b}(\hat{\theta}(\tau))
\end{array}\right]\right\} . d \tau \\
& \hat{i}_{a}+\hat{i}_{b}+\hat{i}_{c}=0
\end{aligned}
$$


where

$$
\begin{aligned}
& \hat{A}\left(\hat{\theta}, i_{d}, i_{q}\right)=\left[\begin{array}{cc}
\hat{L}_{a a}-\hat{L}_{a c}-\hat{L}_{b a}+\hat{L}_{b c} & \hat{L}_{a b}-\hat{L}_{a c}-\hat{L}_{b b}+\hat{L}_{b c} \\
\hat{L}_{b a}-\hat{L}_{a c}-\hat{L}_{b c}+\hat{L}_{c c} & \hat{L}_{b b}-\hat{L}_{b c}+\hat{L}_{c c}-\hat{L}_{b c}
\end{array}\right] \\
& \hat{B}(\hat{T})=\left[\begin{array}{cc}
-r_{a}(\hat{T}) & r_{b}(\hat{T}) \\
-r_{c}(\hat{T}) & -r_{b}(\hat{T})-r_{c}(\hat{T})
\end{array}\right]
\end{aligned}
$$

In the above equations, $\hat{A}$ is the equivalent inductance matrix which was initially calculated offline from transient $\mathrm{FE}$ analysis with motion. Here, $\hat{B}$ is the equivalent resistance matrix it is estimated resistance by the thermal network. Also, $\left(i_{d}, i_{q}\right)$ is the equivalent saturation level. The $L$ demonstrates the inductance symbol, and ' $r$ ' is the resistance symbol, with the indices ' $a, b$ and $c$ " belongs to the phase names. ' $\tau$, is the integration index. ' $V$ ' is the voltage index, and $E$ is the back EMF index. The superscript '*' denotes a command variable. The hat "^’ indicates the estimated variables.

In terms of the difference between the estimated and measured currents, the EMF was estimated as:

$$
\left[\begin{array}{l}
\hat{E}_{a} \\
\hat{E}_{b} \\
\hat{E}_{c}
\end{array}\right]=K_{z} \cdot \omega \cdot\left(K_{p}+\frac{K_{i}}{s}\right)\left[\begin{array}{l}
\operatorname{sign}\left(\hat{i}_{a}-i_{a}\right) \\
\operatorname{sign}\left(\hat{i}_{b}-i_{b}\right) \\
-\hat{E}_{b}-\hat{E}_{a}
\end{array}\right]+\frac{1-e^{-T_{d} \cdot s}}{T_{r} \cdot s} \cdot\left[\begin{array}{l}
\hat{E}_{a} \\
\hat{E}_{b} \\
0
\end{array}\right]
$$

Here, the term $\left(1-e^{-T_{d} \cdot s}\right) /\left(T_{r} . s\right)$ is a linear mean approximation that was added to resolve the chattering problem in a digitalized control system with finite switching frequency, $T_{r}$ determines the running window width in the frequency domain, " $S$ " was the Laplace operator, $T_{d}$ was the dead-time, $\omega=P . \omega_{m} / 2$ was the electrical angular velocity. The superscript '*' denotes a command variable. The hat "^, indicates the estimated variables. $\omega_{m}(\mathrm{rad} / \mathrm{s})$ was the mechanical angular velocity. $t$ 
was the time index. $K_{p}$ and $K_{i}$ are the gains of the PI controller. Also, $K_{z}$ was the input gain.

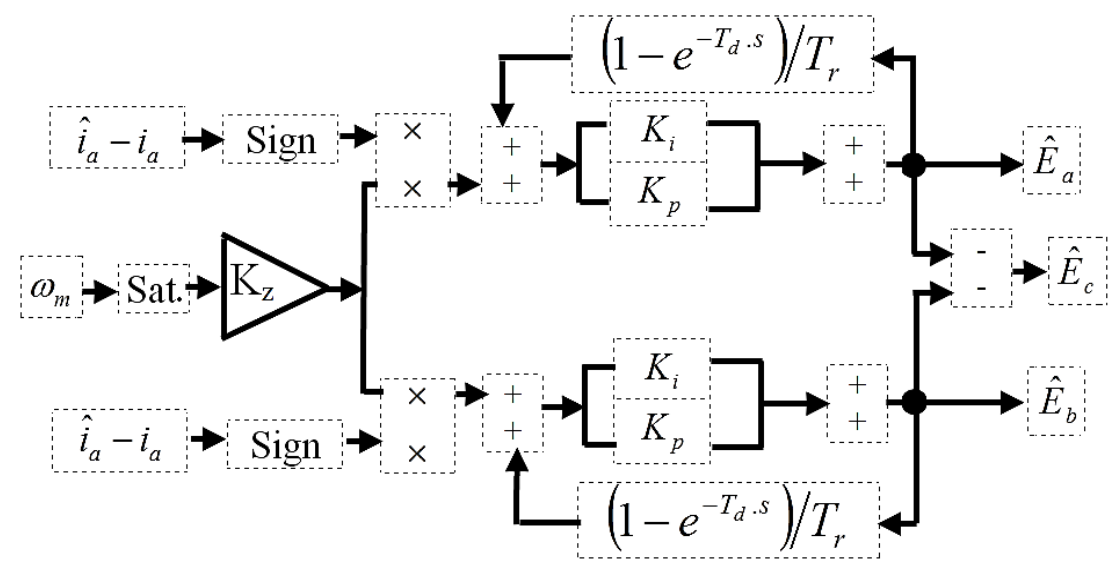

Figure 6.1 Schematic diagram of equation (6-5)

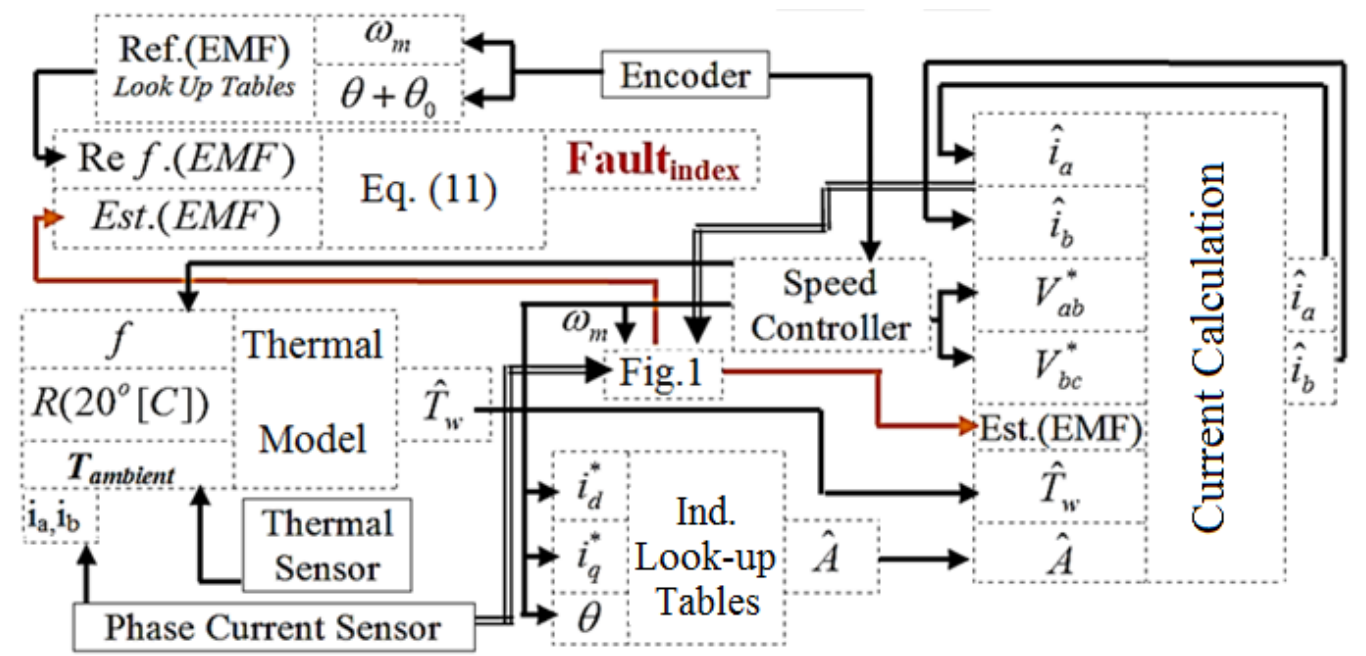

Figure 6.2 Schematic diagram of the whole EMF-based diagnosis system

\subsubsection{Stability criteria of the estimator}

By subtraction of equation (6-1) from the machine equation (3-170) and assuming $V_{a b}=V_{a b}^{*}, V_{b c}=V_{b c}^{*}, A\left(\theta, i_{d}, i_{q}\right)=\hat{A}\left(\hat{\theta}, i_{d}, i_{q}\right), B(T)=\hat{B}(\hat{T})$, the faulty part does not exist and $(d A / d t)=0$, the motion equation was expressed as: 


$$
\dot{M}=A\left(\theta, i_{d}, i_{q}\right) \cdot\left\{B(T) \cdot M+\left[\begin{array}{l}
-\hat{E}_{a}+E_{a}+\hat{E}_{b}-E_{b} \\
-\hat{E}_{b}+E_{b}+\hat{E}_{b}-E_{b}
\end{array}\right]\right\}
$$

Where motion, $M$, is defined as:

$$
M=\left[\begin{array}{l}
\hat{i}_{a} \\
\hat{i}_{b}
\end{array}\right]-\left[\begin{array}{l}
i_{a} \\
i_{b}
\end{array}\right]
$$

For a healthy PMSM one can assume that:

$$
\begin{aligned}
& A^{-1}\left(\theta, i_{d}, i_{q}\right)=\hat{A}^{-1}\left(\theta, i_{d}, i_{q}\right)=\left[\begin{array}{cc}
\frac{4}{9 L_{s}} & \frac{2}{9 L_{s}} \\
\frac{-2}{9 L_{s}} & \frac{2}{9 L_{s}}
\end{array}\right] \\
& B(T)=\hat{B}(\hat{T})=\left[\begin{array}{cc}
-R & R \\
-R & -2 R
\end{array}\right]
\end{aligned}
$$

Using (6-6), by the definition of the Lyapunov function as $V=\left(M^{T} \cdot M\right) / 2$, the necessary criteria for the stability of the estimator was calculated as:

$$
\frac{K_{z} \cdot \omega \cdot\left(K_{p}+\frac{K_{i}}{s}\right)}{1+\frac{e^{-T_{d} \cdot s}-1}{T_{r} . s}}>\frac{\left|E_{a a^{\prime}}-E_{b b^{\prime}}\right|_{\max }}{\sqrt{3}}
$$

From equation (6-10), one can choose $K_{p}=1$ and $K_{i} \geq \frac{e^{-T_{d} \cdot s}-1}{T_{r} . s}$ then in order to guarantee the stability $K_{z}>\frac{\left|E_{a a^{\prime}}-E_{b b^{\prime}}\right|_{\max }}{\sqrt{3} \omega}$. Knowing that $e^{-T_{d} \cdot s}<1$, the $K_{i}>0$ always satisfies the stability criterion. However, in practice for implementation of the term $e^{-T_{d} \cdot s}$ the usage of n-order approximations was usual. Here, the Pade approximation was used.

The n-order Pade approximation of the time delay, $e^{-T_{d} \cdot s}$, was expressed by equation (6-11). The step response of this approximation for the $T_{d}$ equal to $2(\mathrm{~ms})$ 
was illustrated in figure 6.3.a. The enlarged view of figure 6.3.a in the vicinity of peak was depicted in figure 6.3.b. It was seen that, the 1st order approximation satisfies the assumed condition for the time delay i.e. $e^{-T_{d} \cdot s}<1$. However it has has a relatively large setting time. For this reason the higher approximation orders are preferred to lower orders concerning the computational time step. Unfortunately, the higher approximation orders create a small overshoot in the response of the approximated delay. The higher order of approximation, the higher overshoot will be. The maximum overshoot for a 5 th-order approximation was around 2.5 percent, see figure 6.3.b. This overshoot pushes the system to unstable areas because the assumed condition, $e^{-T_{d} \cdot s}<1$, was not valid during the overshoot from the beginning. To resolve this issue, the choice of $K_{i} \geq \frac{O_{s}}{T_{r}}$ was necessary to guarantee the stability of the EMF observer.

$$
P_{n, T_{d}}(s)=\frac{1-\frac{T_{d} s}{2}+p_{2}\left(T_{d} s\right)^{2}+(-1)^{n+1} P_{n}\left(T_{d} s\right)^{n}}{1+\frac{T_{d} s}{2}+p_{2}\left(T_{d} s\right)^{2}+P_{n}\left(T_{d} s\right)^{n}}
$$

Finally, for $K_{p}=1$ if $K_{i} \geq \frac{O_{s}}{T_{r}}$ and only if the gain $K_{z}$ become larger than the maximum phase EMF to neutral at the speed of one $(\mathrm{rad} / \mathrm{s})$ the stability of the estimator was guaranteed. The assumed conditions are stable during the short-circuit fault as well because the inter-turn fault will never lead to a phase EMF increase.

\subsection{Fault index}

The difference between the estimated and the reference EMF can be used in many ways as the fault index. Linear average value of the EMF differences normalized with mechanical angular velocity was introduced as the fault index as: 


$$
\text { Fault }_{\text {index }}=\frac{u(t) \cdot\left(1-e^{-T_{0} \cdot s}\right)}{\left(T_{0} \times \omega_{m}\right) \cdot s}
$$

where $T_{0}$ was the averaging period, " $s$ " is Laplace operator, the $u(t)$ is the difference between the estimated and the reference EMF which is separately defined for each phase as:

$$
u(t)=\hat{E}-E
$$

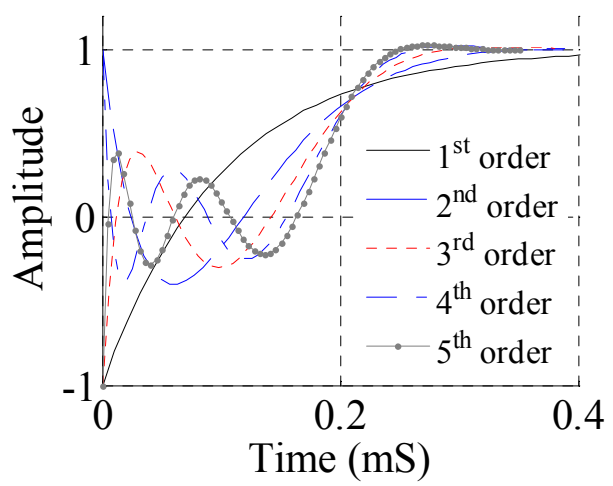

(a)

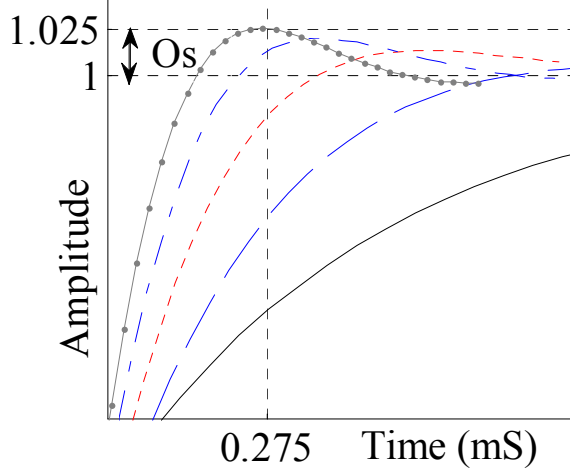

Figure 6.1 Step response of Pade approximation of time delay $\left(e^{-T_{d} \cdot s}\right)$

\subsection{Experimental results}

The studied case was a $0.8(\mathrm{~kW}), 10(\mathrm{Amps})$ PMSM connected to a CSI-based-VSI drive. In PMSM, the resistance per phase is $1.75(\Omega)$ and the maximum speed 20 $(\mathrm{rad} / \mathrm{s})$. The machine's PM is N45. The stator core was made of M19 and the rotor was made of steel 1018. The geometry, dimension and the winding arrangement and flux contour of the machine was presented in figure 6.4. The number of conductors per slot was 38. The winding factor was around 0.6; the phase side flux linkage is illustrated in figure 6.5.a. The cogging torque was illustrated in figure 6.5.b. The self and mutual inductance of phase "a" and phases "a-b" at different saturation levels are depicted in figures 6.5.c and 6.5.d, respectively. As seen in these figures, the inductances are function of the rotor position, $\mathrm{i}_{\mathrm{d}}$ and $\mathrm{i}_{\mathrm{q}}$. The other phase's self and 
mutual inductances have $120^{\circ}$ electrical degree phase shift. The inductances are collected in 3-D look-up Tables. The rotor position was measured by a magnetic encoder and $i_{d}^{*}$ and $i_{q}^{*}$ are measured from the current control loop located inside the speed controller, respectively. The winding temperature was simultaneously updated from the solution healthy transient thermal model of the machine. Using equation (3170), the windings resistances was calculated at the same time as well. For tuning the estimator, in equation (6-10), $K_{z}>20, K_{p}=0.1, K_{i}=100, T_{r}=0.5(m s)$ and $T_{d}=0.1(\mathrm{~ms})$. The PWM switching frequency was $10(\mathrm{kHz})$. The $\mathrm{T}_{0}$ in the equation (6-11) was one second. The sampling frequency was $20(\mathrm{kHz})$. Here, $F_{s}$ was defined as the number of shorted turns in the faulty phase over the total number of turns per phase. $R_{f s c}$ was the fault resistance. The maximum operating speed and the experiment duration was carefully chosen using computer simulations to avoid machine damage during experiment. The dSPACE1103 was used for estimator. The dSPACE1104 was used for the speed controller. By connecting different ISCF leads with different resistance, $R_{f s c}$, it was possible to change the fault intensity.

In the first experiment, the $F_{s}$ was fixed to 0.49 and the fault resistance $R_{f s c}$ was changed from infinity to low values. The $F_{s}=0$ and/or $R_{f s c}=\infty(\Omega)$ was defined as no fault (NF) situation, the $F_{s}=0.49, R_{f s c}=10(\Omega)$ was defined as low fault (LF) intensity situation. Here, the $F_{s}=0.49, R_{f s c}=5.6(\Omega)$ is defined as medium fault (MF) intensity situation. Also, $F_{s}=0.49, R_{f s c}=1.8(\Omega)$ is defined as high fault (HF) intensity situation and the $F_{s}=0.49, R_{f s c}=0.05(\Omega)$ is defined as very high fault (VHF) intensity situation. 
Figures 6.6.a to 6.6.d, figures 6.7.a to 6.7.d, figures 6.8.a to 6.8.d and figures 6.9.a to 6.9.d show the experimental results for the estimated versus the reference EMF in the faulty phase for different fault intensities and for the respective mechanical speeds of $0.75,2,4$ and $8(\mathrm{rad} / \mathrm{s})$. Physically, the fault intensity was associated with the flux linkage per turn, the electrical resistance per turn, speed, fault resistance and the selfinductance of each turn.

The difference between the estimated and the reference EMF in the faulty phase can be considered as the fault indicator. By comparison of figures 6.6 to 6.9 , one can state that the fault indicator was responsive for wide speed range.

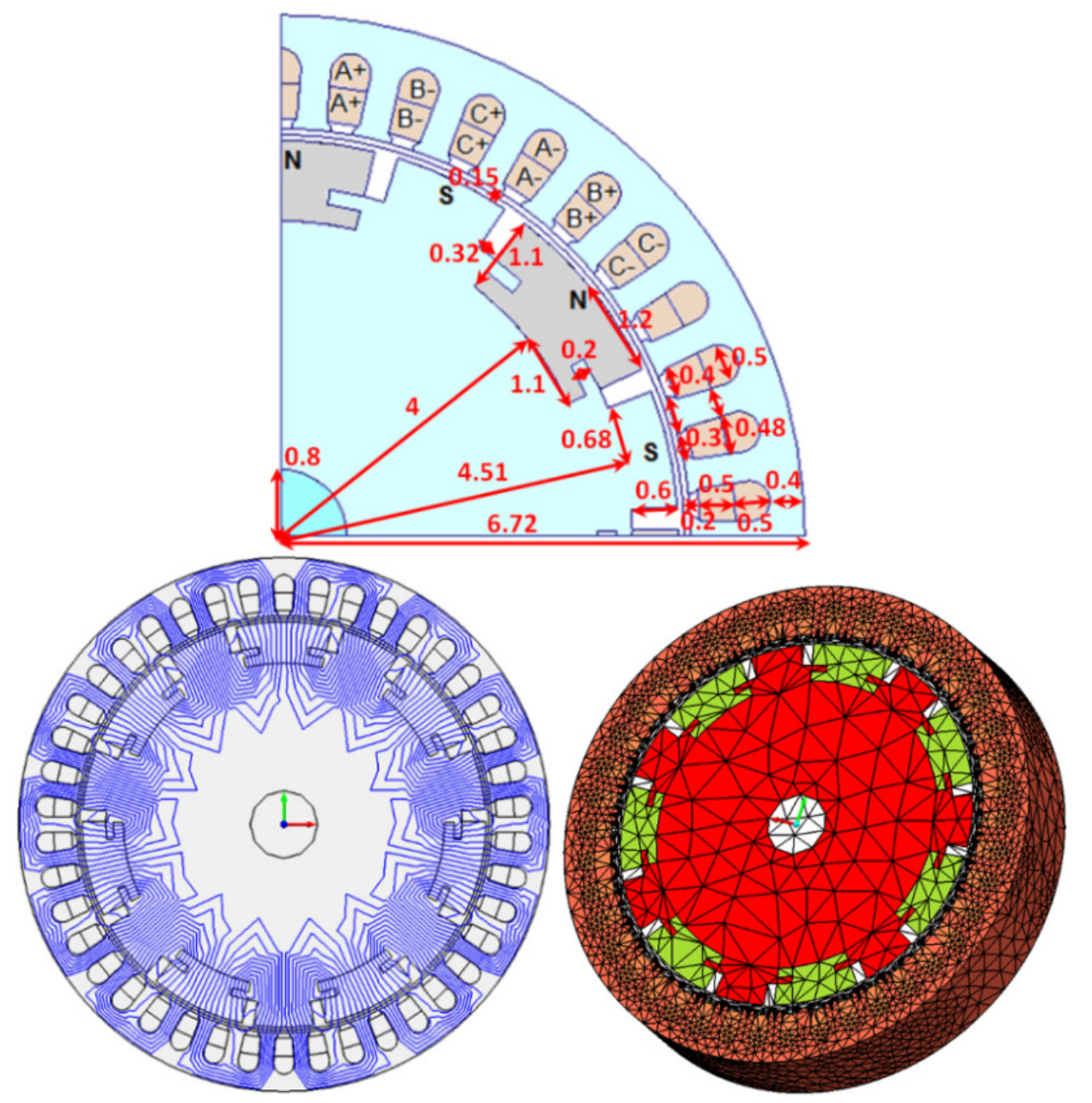

Figure 6.2 The geometry of the machine in $(\mathrm{cm})$ and the winding arrangement 
The fault detection system was sensitive to the intensity of the fault. The higher fault intensity, the higher fault indicator will be.

At low speeds, the Ohmic voltage drops may be dominant to the EMF voltage drops especially at high currents and high ambient temperature. Therefore, the difference between the estimated and actual EMF is more influenced by the differences between the actual machine resistance and the resistance estimated from the thermal model.
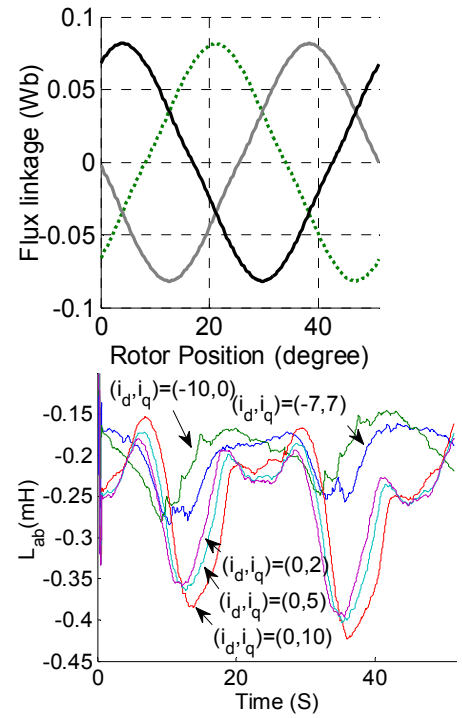

(a)

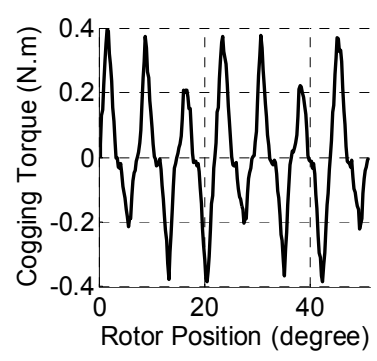

(c)

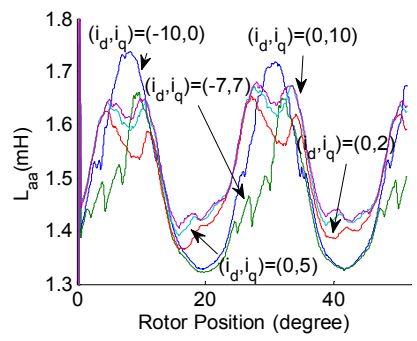

(b)

Figure 6.3 flux linkage(a), Cogging Torque (b), $\mathrm{L}_{\mathrm{aa}}$ at different saturation levels(c) and $\mathrm{L}_{\mathrm{ab}}$ at different saturation levels (d)

The shorted turn's EMF, shorted turns resistive and inductive voltage drops are by passed by resistance, $R_{f s c}$, during the fault. Therefore, the fault indicator value may be interpreted in three ways. The summation of the shorted turn's voltage elements was a positive voltage that circulates a negative $i_{f}$ inside the $R_{f s c}$. This causes an overall voltage drop equal to $R_{f s c} \times i_{f}$. The amount of the fault indicator value was a direct consequence of $R_{f s c} \times i_{f}$. 
Secondly, the reverse flux linkage produced by the shorted turns opposes the PM flux linkage to reduce the flux linage variations in the shorted turns, i.e. ultimately it tries to completely cancel out the shorted turn flux linkage variations. This reverse flux was created by the inductive part of the shorted turns. It also reduces the PM flux linkage in the healthy parts in the faulty phase which are out of phase with the shorted turn's reverse flux. This decrease was directly reflected on the fault indicator in all phases. In addition, worse condition happens when the summation of inductive voltage drop of the shorted turn tries to completely cancel out the shorted turn flux linkage variations. In this case, a significant performance variation occurs through a significant EMF reduction in the healthy parts as well.

The reasons for the changes in the estimated EMF in the healthy phases due to inter-turn fault in faulty phase may be interpreted in three ways.

Related to the Ohmic voltage drops, after an overall machine temperature rise due to the ISCF the actual machine resistance becomes higher than what was estimated from healthy thermal model used in estimator and therefore the resistive voltage drop in the healthy phases of the actual machine would be higher than that of the model given the machine and the model voltage terminals and phase currents are the same. As a result, the estimated EMF from the model in the healthy phases will be higher than that of the actual machine EMF. This effect was more visible at low speeds because the resistance voltage drops was generally dominant over the machine EMF.

Due to decreased saturation level that happens in vicinity of the shorted turns inside the iron parts, the actual inductances of the phases become higher than the estimated inductance. In this situation, the inductive voltage drop of the actual machine would be higher than that of the model and as a result the estimated EMF in the healthy phase from the model will be higher than that of the actual machine EMF 
required the machine and the model currents are the same. This effect was more visible when the flux weakening control was weak. This increase was indirectly reflected on the estimated EMF.

The reverse flux linkage produced by the shorted turns partially opposes the PM flux linkage in healthy phases. This decrease was directly reflected on the estimated EMF.

In conclusion, it can be said that in the healthy phases the inductive and resistive voltage drops effects increase the estimated EMF in healthy phase while the reverse flux and effect decrease the estimated EMF in healthy phase. The resultant influence of these positive and the negative portions was related to the machine characteristics, speed, fault intensity, ambient temperature and the time constant of temperature rise. During experiments it was seen that the reverse flux effects are dominant to the inductive and resistive voltage drops and as a result the EMF in faulty phases are decreased as well. The experimental result of this statement was illustrated in figure 6.10. In this figure the phase "b" was faulty and the phases "a" and "c" are healthy.

In addition, it was observed that the method was robust with respect to the harmonics presented in the speed and current. figure 6.11.a, 6.11.b and 6.11.c respectively show the estimated versus reference EMF, machine speed and phase currents for a non-linear and non-periodic harmonic load condition. In this figure the reference speed was $2 \mathrm{rad} / \mathrm{s}$ but due to the non-linear harmonic load, the speed closes to zero in some time intervals. Evidently, the presented non-stationary harmonics in the phase current make the usage of phase current as the fault signature under serious limitation.

The experimental result of the transient behavior of the estimated versus the reference EMF for the speed of $4 \mathrm{rad} / \mathrm{s}$ was illustrated in figure 6.12 . In this figure a 
transient high fault (HF) situation was intentionally added at 0.45 (s) and it was removed at $0.9(\mathrm{~s})$. It was seen that the estimator instantly react to fault.

The operational fault index calculated from equation (6-11) was depicted figure 6.12. In this Figure, two cases were studied for the speed of $3 \mathrm{rad} / \mathrm{s}$. In the case1, the LF with $F_{s}=0.49$ and $R_{f s c}=10(\Omega)$ was appeared at $2(\mathrm{~s})$, another fault with $F_{s}=0.1$ and $R_{f s c}=0.15(\Omega)$ was added to it at $6(\mathrm{~s})$. In the case 2 , the LF with $F_{s}=0.49$ and $R_{f s c}=10(\Omega)$ was appeared at $2(\mathrm{~s})$, another fault with $F_{s}=0.05$ and $R_{f s c}=0.15(\Omega)$ was added to it at 6(s). It was seen that the fault index was sensitive to the low number of the shorted turns. In addition, the number of the shorted turns was reflected on the fault index.

In order to compare the usage of the EMF, current, or voltage as the fault signature, the respective experimental result of the voltage terminal and the phase current of the Figures 6.6.b and 6.9.b was illustrated in figure 6.14.a, 6.14.b, 6.14.c and 6.14.d. The comparison of these Figures generally reveals that the EMF variation was more sensitive to the fault than the voltage and current. The EMF and the current are more responsive to fault at high speeds. Similar conclusion was valid for higher fault intensity conditions. It was mentioned that in the studied case, some unwanted harmonics was presented in current. It is due to the natural dynamic eccentricity in PMSM, low resolution of encoder specially at low speeds and high cogging torque in the studied PMSM. 


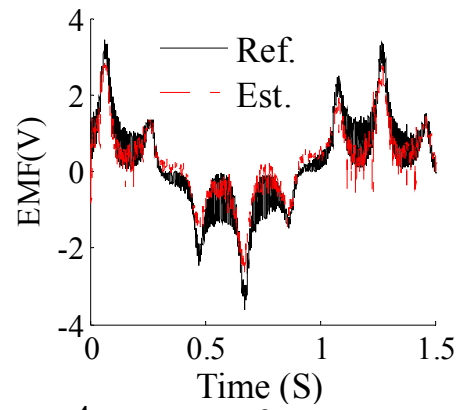

(a)

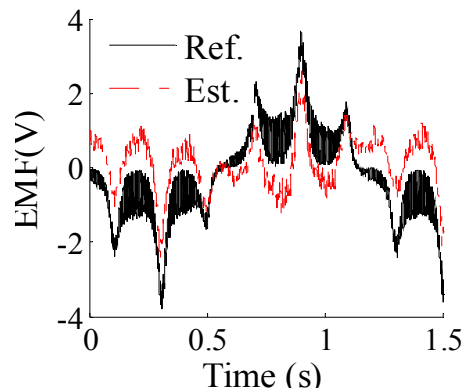

(b)

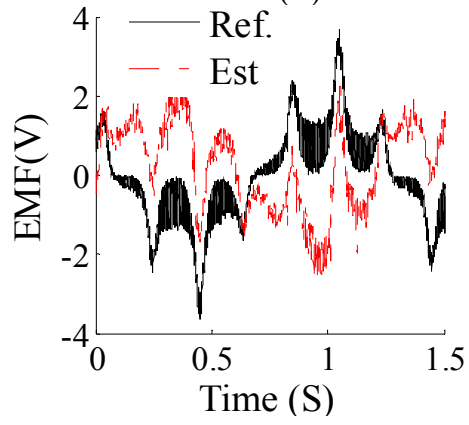

(c)

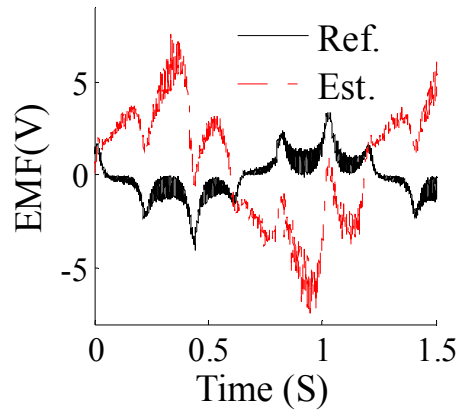

(d)

Figure 6.4 Reference versus estimated EMF in faulty phase with different fault intensity, NF (a), LF (b), MF (c), HF (d), all for speed of $0.75 \mathrm{rad} / \mathrm{s}$

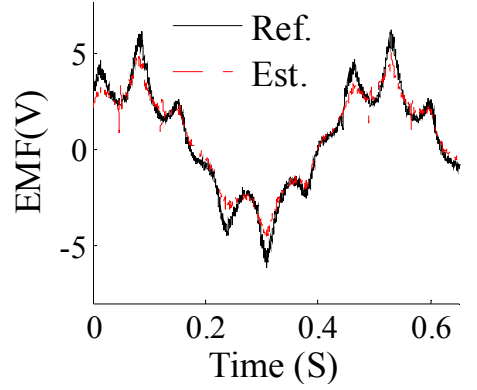

(a)

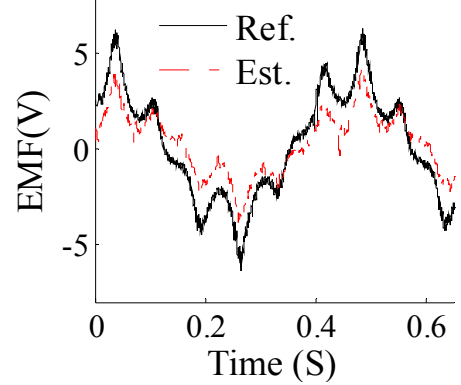

(b)
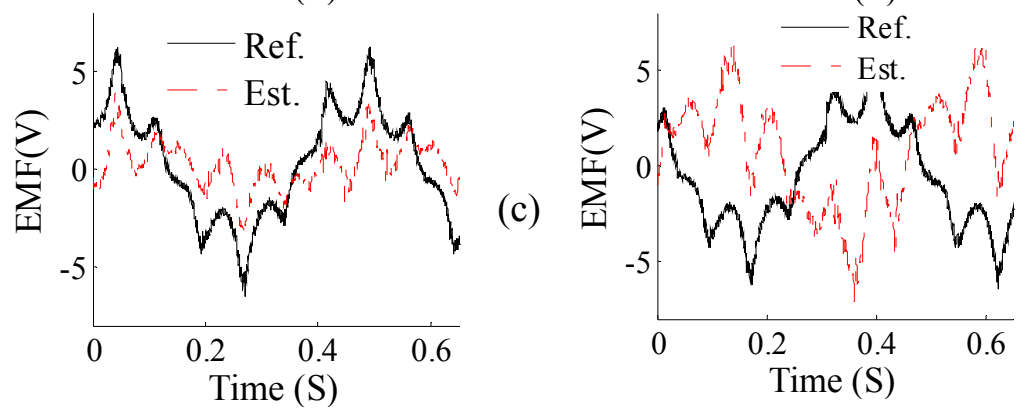

(d)

Figure 6.5 Reference versus estimated EMF in faulty phase with different fault intensity, NF (a), LF (b), MF (c), HF (d) all for speed of $2 \mathrm{rad} / \mathrm{s}$ 


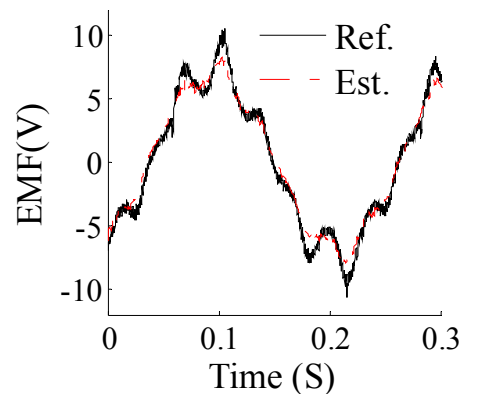

(a)

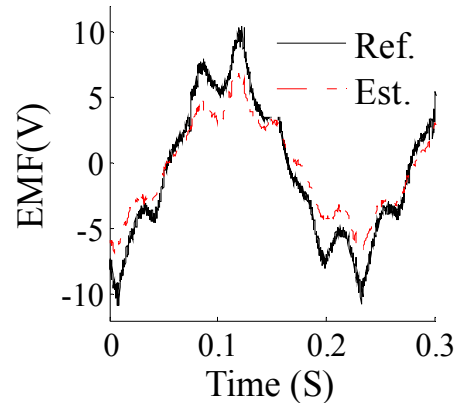

(b)
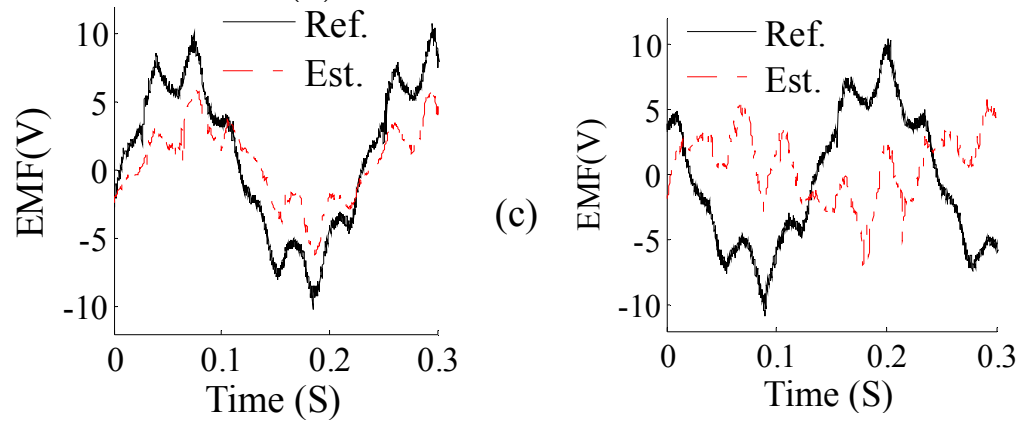

(d)

Figure 6.6 Reference versus estimated EMF in faulty phase with different fault intensity, NF (a), LF (b), MF (c), HF (d) all for speed of $4 \mathrm{rad} / \mathrm{s}$

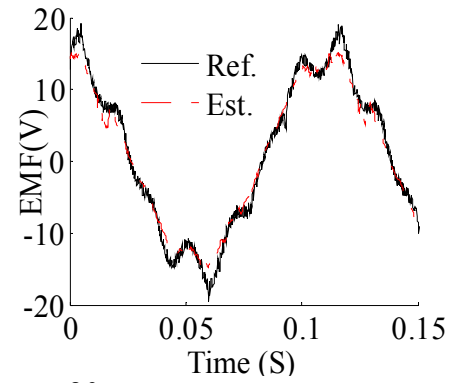

(a)

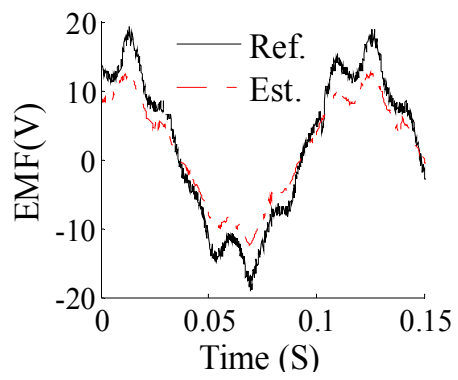

(b)
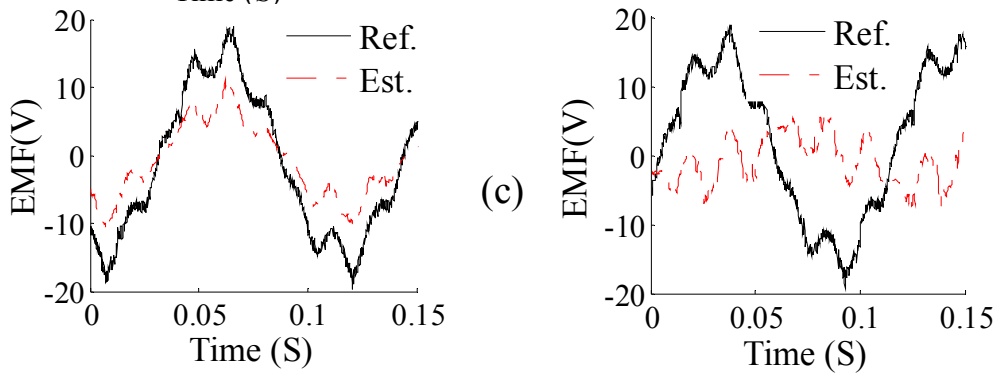

(d)

Figure 6.7 Reference versus estimated EMF in faulty phase with different fault intensity, H (a), LF (b), MF (c), HF (d) all for speed of $8 \mathrm{rad} / \mathrm{s}$ 


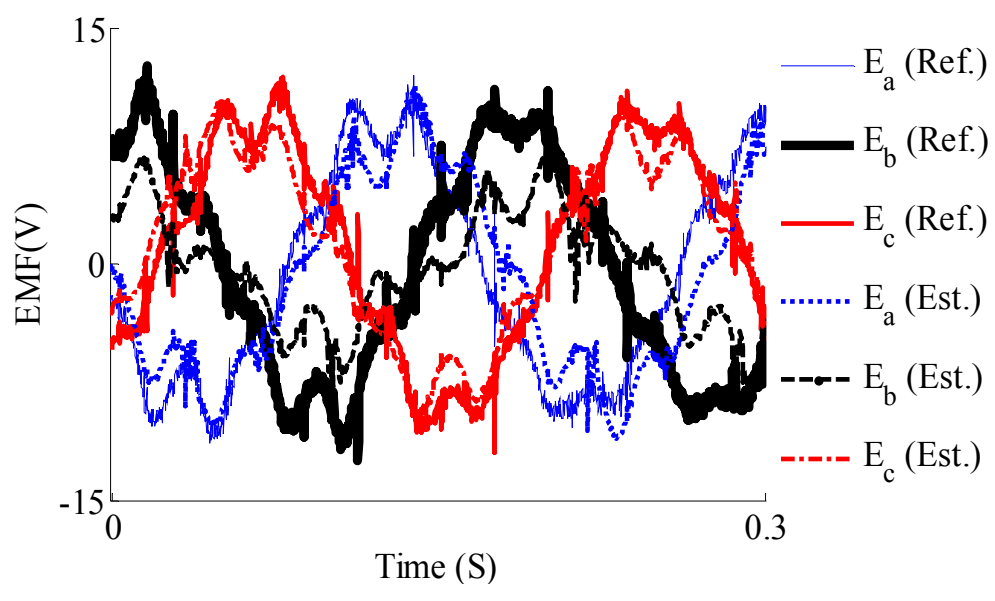

Figure 6.8 Reference versus estimated EMF with MF fault intensity at phase "b" for the speed of $5 \mathrm{rad} / \mathrm{s}$

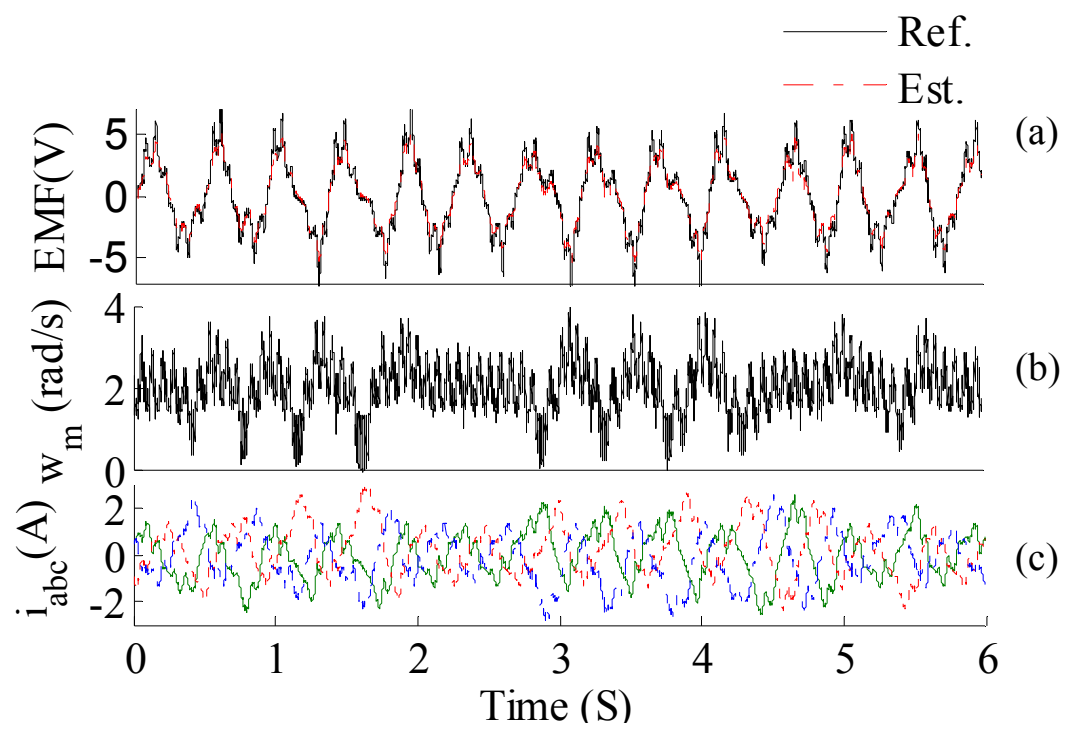

Figure 6.9 Comparison of estimated versus reference EMF for a harmonic load

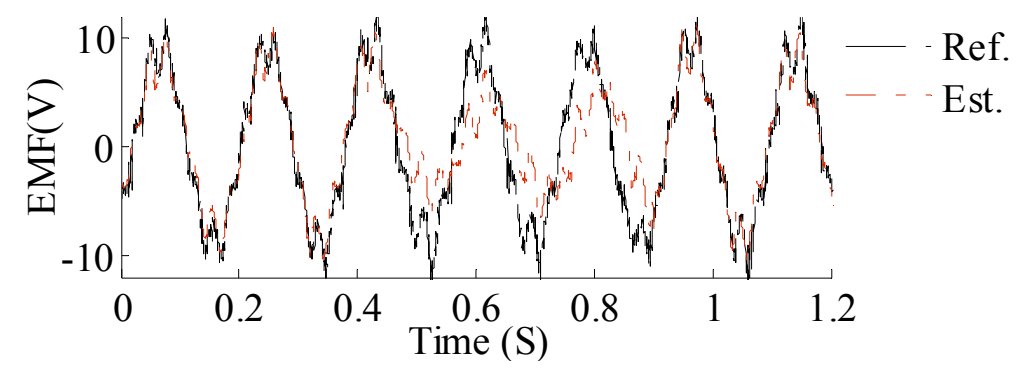

Figure 6.10 Reference versus estimated EMF for a transient HF intensity 


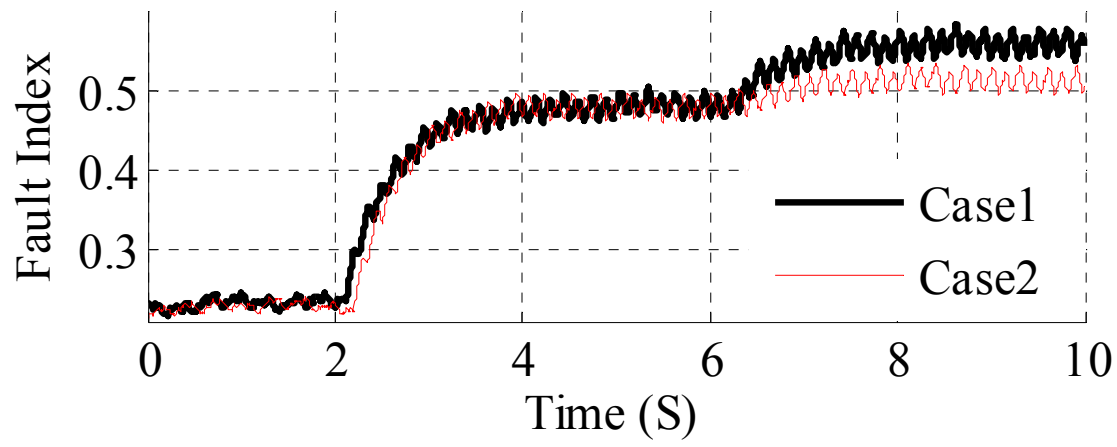

Figure 6.11 Transient fault index for two successive LF situations at 2(s) and 6(s) for case 1 and 2 .both for $25 \%$ of nominal torque

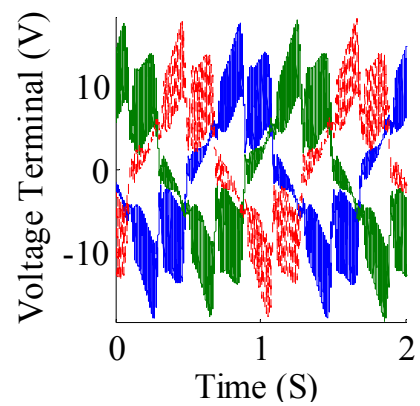

(a)
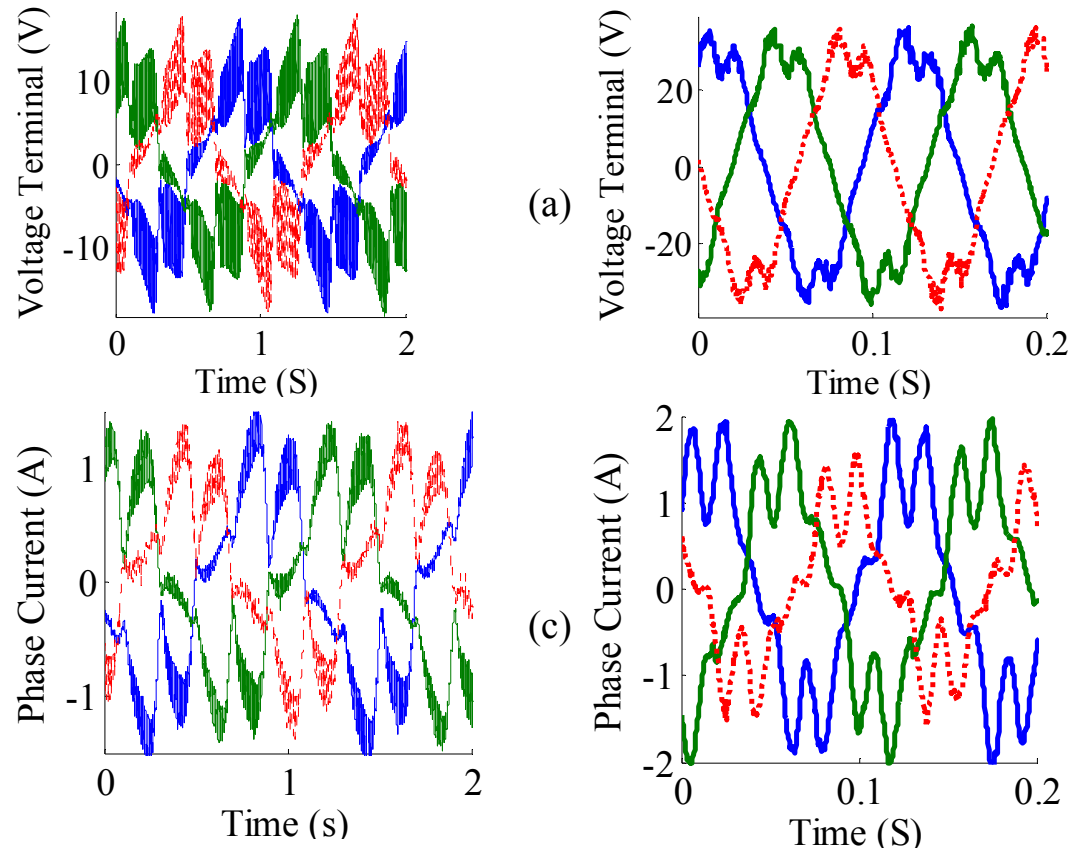

(c)

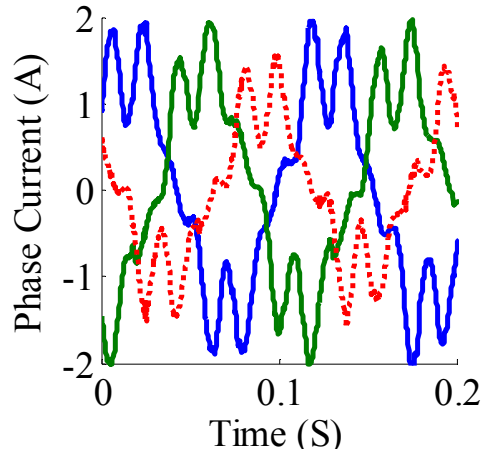

Figure 6.12 Voltage terminal and phase current for LF intensity condition at two different speed $0.75(\mathrm{rad} / \mathrm{s})(\mathrm{a}, \mathrm{b})$ and $8(\mathrm{rad} / \mathrm{s})(\mathrm{c}, \mathrm{d})$

The experimental and simulated result of the transient behavior of the EMF estimation system during slow and fast speed transients was shown in figure 6.15.a to 6.15.d. It was seen that during the speed transients, the reference was perfectly tracked by the estimation. The simulation result with respect to figure 6.9 was illustrated in figure 6.16. The comparison of these two Figures proves the accuracy of the modeling. 
The method is the sensitive to the DC bus variation. The reason is that the used reference DC value in the evaluation of the duty cycles from the reference voltages was fixed. This can be solved by measuring the DC bus voltage and then multiplying the reference voltage to the ratio between the fixed and actual DC voltage.
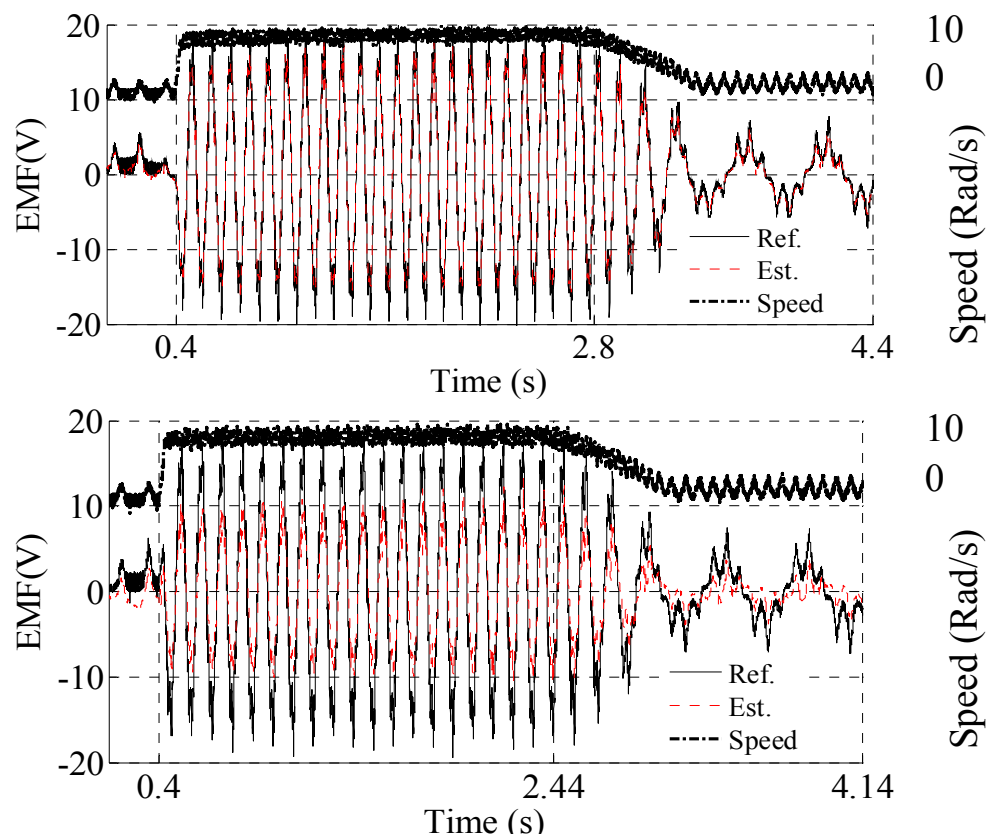

(b)

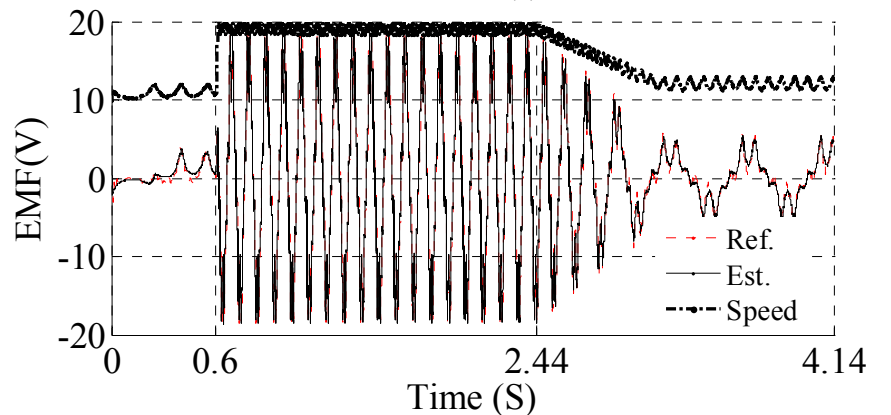

10

0

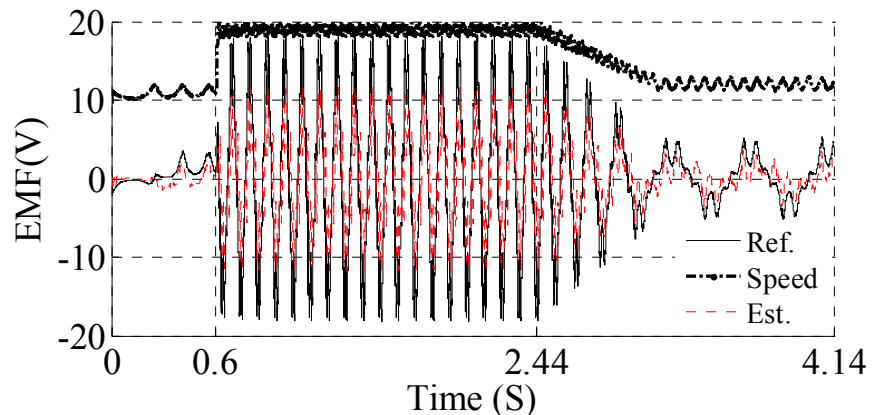

10

0

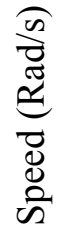

(d)

Figure 6.13 Reference versus estimated EMF during fast and slow speed variations, (a) and (b) experimental results, (c) and (d) simulation results 


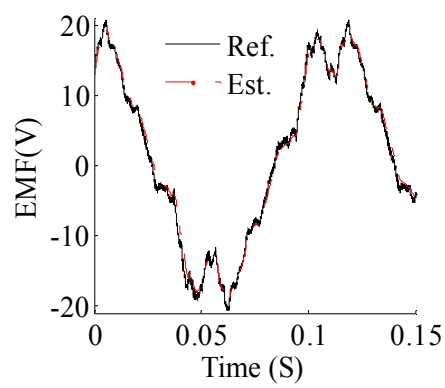

(a)

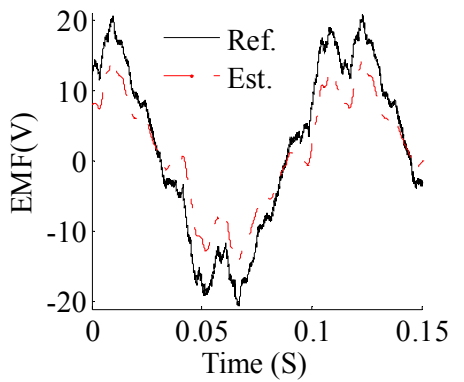

(b)
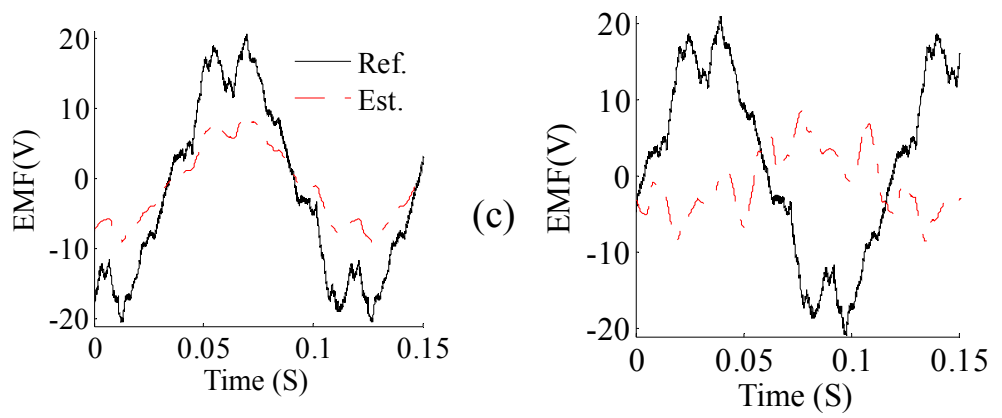

(d)

Figure 6.14 Reference versus estimated EMF in faulty phase with different fault intensity, H (a), LF (b), MF (c), HF (d) all for speed of $8 \mathrm{rad} / \mathrm{s}$

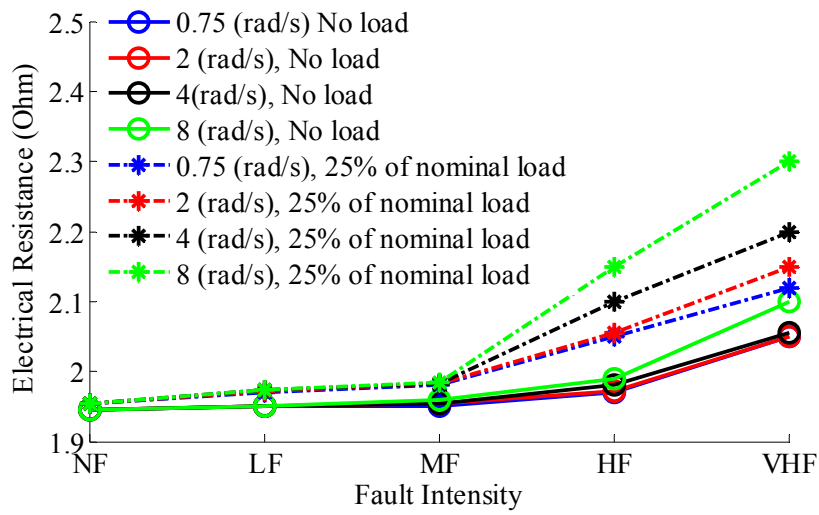

Figure 6.15 Estimated electrical resistance as a function of speed and fault intensity for no load (circles) and $25 \%$ of nominal load (stars) conditions

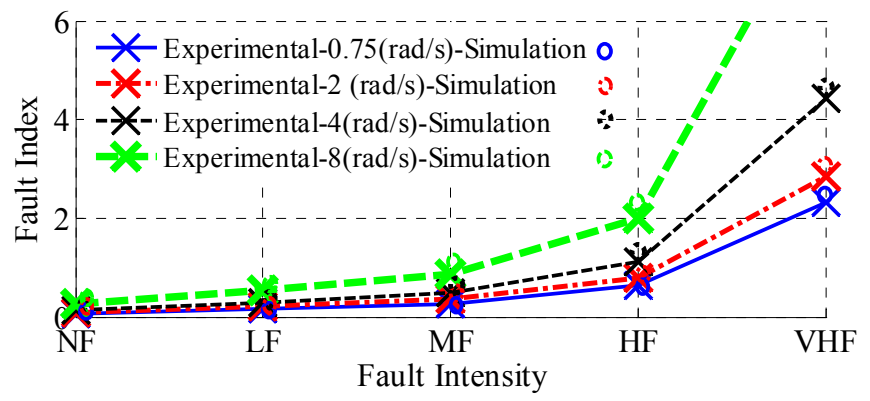

Figure 6.16 Fault index as a function of speed and fault intensity all for $25 \%$ of nominal torque 
Inter-turn fault is easier to detect leading to shorter time to breakdown. Figure 6.17 shows the experimental results of the estimated electrical resistances at steady state conditions. The tolerance of the estimated resistance was between $\pm 2 \%$ to $\pm 5 \%$. The operational winding temperature was obtained from the thermal network. Here the ambient temperature was fixed. From figure 6.17 it was observed that the estimated resistance was sensitive to inter-turn faults where in general the estimated resistance was strictly ascending at higher fault intensities. However, speeds and/or the loading condition also affect the estimated resistance. For example, from figure 6.17 it was seen that the estimated electrical resistance for a NF situation at $25 \%$ of nominal load condition was almost equal to the estimated electrical resistance of an MF situation at no load condition. Furthermore, any increase in the ambient temperature decreases the heat transfer rate and consequently leads to an increase in the electrical resistance. Therefore, the usage of the estimated electrical resistance for fault detection relies on the proper usage of a knowledge-based system.

The simulated and experimental results of the steady state fault index as a function of speed and the fault intensity is depicted in figure 6.18. The fault index was strictly ascending for higher speeds and higher fault intensities where the probability of machine damage increases. The variations in the magnetization due to ISCF affect the resultant EMF the faulty phase.

\subsection{Summary}

The physics-based development of an open loop EMF estimator was presented. The design of FE-based PMSM software models required for initialization of interturn fault studies in a PMSM was explained. The fault detection based EMF estimation due to inter-turn fault was studied and discussed. It was observed that the method was stable in detection against the blurring effects of the harmonic loads and 
dynamic eccentricity for wide speed range. It was also observed that the EMF can determine the intensity and the location of the faulty phase in a fraction of second. This method allows prompt and online stator-fault detection from the real-time estimated EMF without additional heavy computations for signal processing methods. The developed method shows acceptable results for low speeds where the inter-turn fault reflection on the performance of the machine, phase current and voltage terminals are not easily sensible. Finally, the results show acceptable performance in fault detection for a wide speed range even in the presence of harmonic loads and dynamic eccentricities. 


\section{Chapter 7 Optimization of Machine Drive Systems}

\subsection{Design of electromagnetic devices}

The design of electromagnetic devices [194] such as electrical machine is a cooperative and successive process typically accomplished by a team of skilled engineers. The objective of the design process is to produce a device which, in its physical characteristics and performance, meets a predefined set of objectives. Conventionally, this process is associated with the development of accurate physical models to test the design ideas and explore the design space variable. Although robust, this approach is a time consuming and costly approach to developing new devices or systems and can consume a significant percentage of the total costs of a product. In a conventional design process, many aspects of a device must be taken into account from physical characteristic to operational performance and from financial views to legal constraints. Most of these aspects require knowledge based approaches to be implemented. Moreover, most devices physically are placed in interdependences between different area of the science such as the electromagnetic, fluid and solid mechanics. These aspects necessitate the assessment of the computing processes and hardware structures to form the virtual design prototyping environment. The virtual design prototyping environment enables the design team to reflect their skill and expertise into an automatic process. Such an automatic system naturally requires a distributed computational environment. Indeed, the aim of any refined design system is to link the most applicable tools for solving the problem at hand. At the hardware level, the existing data on the technical data-bases, the development of Virtual Private Networks VPN) and the local clusters will provide the architecture on which to write the software model. In the software level, the intelligent systems, the 
hardware in the loop facilities, numerical software and the modern theories of control must be specified based on the need and design ideas. Finally, it is mentioned that the skilled engineer cannot be removed from the design system because in most design problems there are several possible "optimal" solutions. The best solution is better to choose based on the knowledge of the designers. In addition, the artificial decision making process can be used to guide the design process automatically to provide the designer with feasible and easy solutions.

The feedback from the artificial decision making process, the design engineer and the real prototype are needed to reduce the design errors and optimize the design.

Such an approach to the design of an electromagnetic device requires a suitable starting point for an optimal solution using only the basic laws of physics and some rough knowledge of material behavior.

The design of permanent magnet machines is an empirical, application sensitive, iterative and systematic process. In the design process, the computer-aided design of the PM machine either usually starts from the selection of the initial geometry and material from an existing machine design that has generally a close input-output performance to the desired machine. This is done normally using a case base reasoning (CBR) systems[195], or it is started by calculation of the initial geometry or material from classic design procedures. Obviously, these procedures might lead to non-optimal designs that the designer expects to succeed and therefore an iterative change of the initial design parameters supervised by a skilled engineering team is needed to optimize the design. Alternatively, this process can also be done through an artificial intelligence optimization-decision making an algorithm to improve the initial design[104]. Clearly, the second approach is attractive because it is less time consuming and less expensive approach to developing new devices or system. The 
optimization process can set concerning different technical and non-technical objectives. Some of these objectives are in similar direction and some of them are in opposite direction. These include the electrical and thermal, cost and reliability, magnetic and mechanical and environmental and packaging in addition to other objectives.

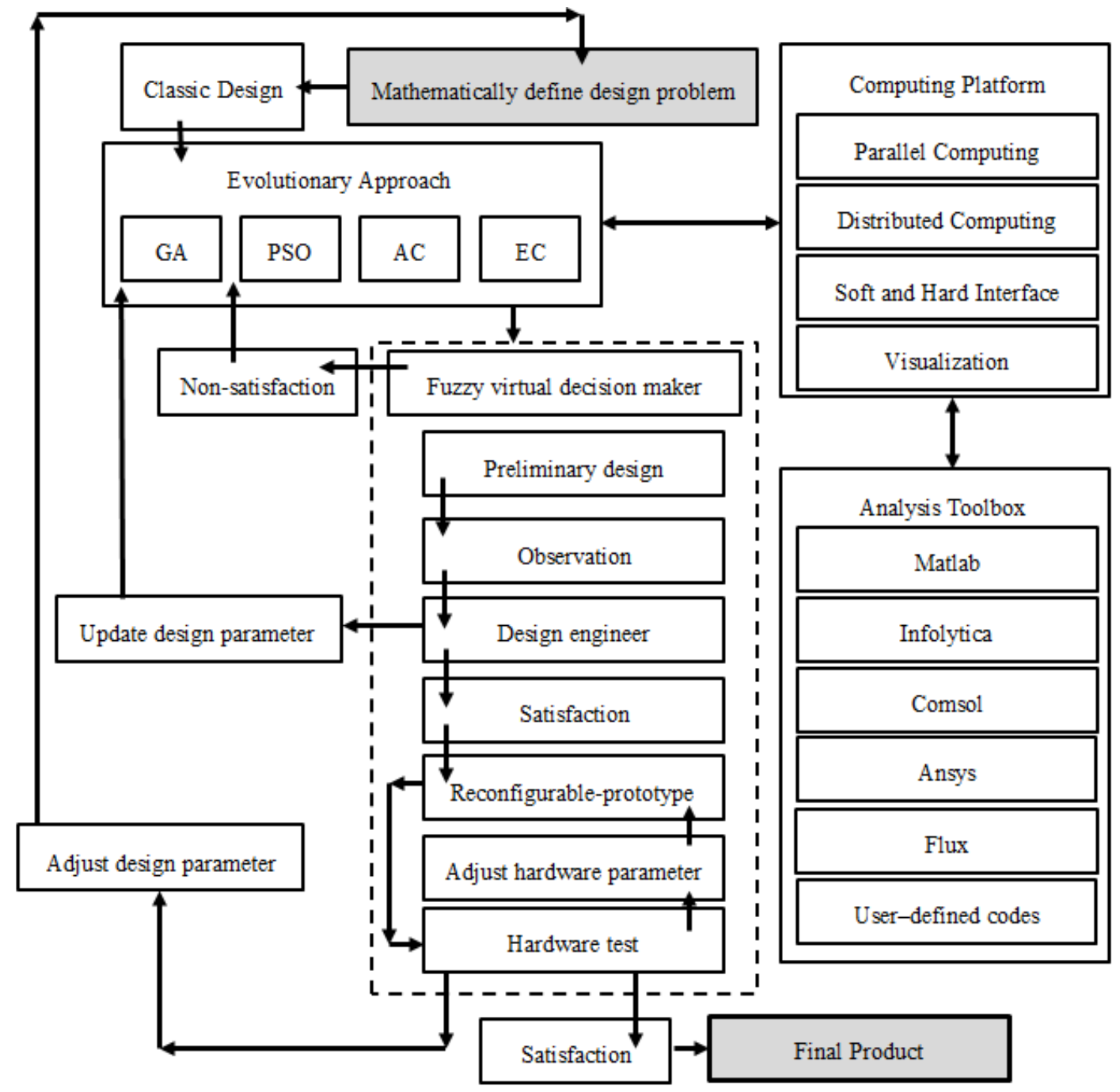

Figure 7.1 Computational design prototyping of electromagnetic devices[194]

\subsection{Optimization based evolutionary algorithms}

\subsubsection{The hybrid GA-PSO}

Particle swarm optimization (PSO)[196] is a population-based optimization 
algorithm that explore for the best solution by simulating the movement and flocking of birds. The Particle swarm optimization (PSO) is launched by initializing of $\mathrm{N}$ set of n-dimensional flock of birds randomly over the n-dimensional searching space. For a n-dimensional search space, the $i^{\text {th }}$ particle, can be represented by an $n$-dimensional vector, $X_{i}=\left[x_{i 1}, x_{i 2}, \ldots, x_{i n}\right]^{T}$ and velocity $V_{i}=\left[v_{i 1}, v_{i 2}, \ldots, v_{i n}\right]^{T}$. Therefore the total size of each population is an $\mathrm{N}$ by $\mathrm{N}$ matrix.

In PSO, the best position that the particle " $i$ " visited so far, referred to as $P_{i}=\left[p_{i 1}, p_{i 2}, \ldots, p_{i n}\right]^{T}$ and the best position of the best particle in the swarm is referred to as $G=\left[g_{1}, g_{2}, \ldots, g_{n}\right]^{T}$. Each particle " $i$ " can adjust its position in the next iteration “ $t+1$ " with respect to equations (7-1) and (7-2) [196-198]:

$$
\begin{aligned}
& V_{i}(t+1)=\omega(t) \cdot V_{i}(t)+C_{1} \cdot r_{1} \cdot\left[P_{i}(t)-X_{i}(t)\right]+C_{2} \cdot r_{2} \cdot\left[G(t)-X_{i}(t)\right] \\
& X_{i}(t+1)=X_{i}(t)+\chi \cdot V_{i}(t+1)
\end{aligned}
$$

where $\omega(t)$ is the inertia coefficient which is employed to control the effect of the previous history of velocities on the current velocity. Here, $\chi$ is a constriction factor which is used to limit velocity. The parameter $\chi$ is equals 0.71 . Also, $C_{1}$ and $C_{2}$ denote the cognitive and social parameters and $r_{1}$ and $r_{2}$ are random real numbers drawn from uniformly distributed interval $[0,1]$.

Here, $\omega(t)$ is initialized to a large value (assumed 1), giving priority to global exploration of the search space and gradually decreasing to a small value (here, 0 ) as to obtain a refined solution. Here, $C_{1}$ and $C_{2}$ adjust dynamically. Experiments show that we should initialize $C_{1}$ to 2.5 and decrease it monotonically to 1.5 during the optimization procedure. For enhancing the PSO's ability in escaping from local minima, a mutation operator is mixed to the PSO algorithm[198]. At the end of each 
of the iterations, a reselection is also done based upon selection probability of each solution, equation 7-6.

\subsubsection{Multi-objective optimization}

A classical move toward a multi-objective optimization problem [199] is to allocate a weight $\omega_{i}$ to each normalized objective function $Y^{\prime}\left(X_{i}\right)$ so that the optimization problem appear in terms of a single objective problem with a scalar objective function as:

$$
\min (Y)=\omega_{1} \cdot Y_{1}^{\prime}\left(X_{i}\right)+\omega_{2} \cdot Y_{2}^{\prime}\left(X_{i}\right)+\ldots+\omega_{k} \cdot Y_{k}^{\prime}\left(X_{i}\right)+\text { Penalti }
$$

where $Y_{k}^{\prime}\left(X_{i}\right)$ is the normalized objective function of $Y_{k}\left(X_{i}\right)$ and $\sum \omega_{i}=1$. This method is called the priori method since the user is expected to offer the weights. Solving a problem with the objective function (7-3), for a given weight vector $\omega=\left\{\omega_{1}, \omega_{2}, \ldots, \omega_{k}\right\}$,yields a single solution and if multiple solutions are desired, the problem must be solved multiple times with different weight combinations randomly. The main difficulty with this method is selecting a weight vector for each run[199]. The random calculation of weights is provided in step 2.2. The steps for atomizing the whole of the optimization process is summarize as below:

Step 1: Generate " $i$ " sets of random variables as the inputs of optimization process with respect to the initial geometry calculated from the classic design of the machine and their boundaries such as:

$$
X_{i}=\left[x_{i 1}, x_{i 2}, \ldots, x_{i n}\right]^{T}
$$

Step 2: Assign a fitness value to each solution by performing the following steps:

Step 2.1: Generate a random number ' $u_{k}$ ' in $[0,1]$ for each objective indices ' $k^{\prime}$. 
Step 2.2: Calculate the random weight of each objective ' $k$ ' as:

$$
\omega_{k}=\frac{u_{k}}{\sum_{i=1}^{k} u_{i}}
$$

Step 2.3: Calculate the fitness value from equation (7-3)

Step 3: Calculate the selection probability of each solution as:

$$
p(x)=\frac{f\left(X_{i}\right)-f^{\min }}{\sum_{i=1}^{k} f\left(X_{i}\right)-f^{\min }}
$$

where $f^{\text {min }}$ is the minimum value of multi-objective function in each iteration.

Step 4: do the proposed Genetic-particle swarm optimization.

Step 5: If the stopping condition is not satisfied, go to Step 2. Otherwise, return to populations and objective values.

\subsubsection{The discrete search space}

Because the FE-based calculations of the physical characteristics of the IPM machine are a time consuming and the optimization is an iterative task, it is necessary to limit search space. Here, the search space is limited by rounding the value of random variable $X_{i}=\left[x_{i 1}, x_{i 2}, \ldots, x_{i n}\right]^{T}$ as:

$$
X_{i}^{\text {new }}=\operatorname{round}\left(\frac{m \cdot X_{i}}{X_{i}^{\max }-X_{i}^{\min }}\right) \times \frac{X_{i}^{\max }-X_{i}^{\min }}{m}
$$

where $X_{i}^{\max }$ and $X_{i}^{\min }$ are the limitation of variables and $2 \leq m<\infty$ is the space reducer factor. In fact using equation (7-7), the continuous search space is divided into ' $m$ ' discrete search space. A proper choice of ' $m$ ' depends on the accuracy of manufacturing, time restriction for simulation and physics of the problem.

\subsection{Design improvement of PMSM-drive system using physics-based motor}




\section{model and evolutionary approaches}

In design solution categories of the PM machines, it is possible to change the back EMF waveform of a PM machine by changing the geometrical design parameters of the machine or changing the materials. On the other hand, it is recognized that the established current waveform through the machine is mainly proportional to the back EMF waveform. Therefore in the form of an inverse problem, it is possible to change the back EMF waveform for having a more desirable current waveform[200]. The current waveform of the PM machine plays an essential role in the performance of the machine. Firstly, it affects the torque ripple of the machine; secondly, it affects the total harmonic distortion of machine; thirdly, it affects the magnetic losses and, finally it affects the copper losses of the machine and the consumed copper volume.

On the other hand, the torque ripple of a PM machine is originated from three sources i.e. the cogging torque, the mismatching between current waveform and back EMF waveforms and the mismatching between the phase inductances and the phase currents. In general, there are three possible ways to reduce the torque ripple. The first methode is to design the drive of the machine with respect to the maximum compatibility of the phase current to the back EMF of machine. The second method is to design the geometry and material of the machine for the minimum cogging torque [200]. Finally, the third method is to design the geometrical design parameter of the machine with respect to the minimum cogging torque and the most compatible back EMF waveform to the phase current.

From machine design point of view, the back EMF waveform is mainly influenced by the magnetic materials, magnets magnetization (radial, parallel, or etc), magnets geometries and the magnets location in the rotor. On the other hand, in the geometrical solution category where the cogging torque originates from the energy 
variation of the air gap field due to the mutual interaction of the rotor magnets and stator openings (slot openings and teeth), there are various techniques to reduce the cogging torque. Basically the main Principles for reduction of the cogging torque is to reduce abruptness of pole-teeth attraction which can be performed by magnet reshaping, teeth skewing, pole skewing, etc.[101]

The optimization explores the best trade-offs between geometrical design parameters of an PM motor with the motor phase current, back EMF waveform and cogging torque as shown in figure 7.2. This is to achieve minimum torque ripple, minimum RMS value of phase current, minimum total harmonic distortion of phase current and as a result maximum efficiency. An optimization algorithm was developed to optimize a multi- objective function which covers the desirable goals. The input variables of the multi-objective function are defined as the normalized variance of torque ripple, RMS value of phase currents and total harmonic distortion of phase currents. The output of the multi objective function is a fitness value that is sent to optimization algorithm. An interface block is placed between the multi objective function and the optimization block. The interface block consists of a physics-based model of the PM motor presented in chapter 3. It is used to indirectly link the geometrical design parameters of the machine to the drive circuit and output performances. The inputs of the interface block are the geometrical dimension of rotor and stator which mainly affect the magnet and copper volumes. The outputs of this block are the total torque, flux density in a specified location of the machine, current density and the phase currents. The physics-based model is linked to an existing sinusoidal current drive to account for the effects of the drive topology on the performance of the machine. In fact, the current drive is seen like a black box model while its influences on the performance of the machine are taken into account. 


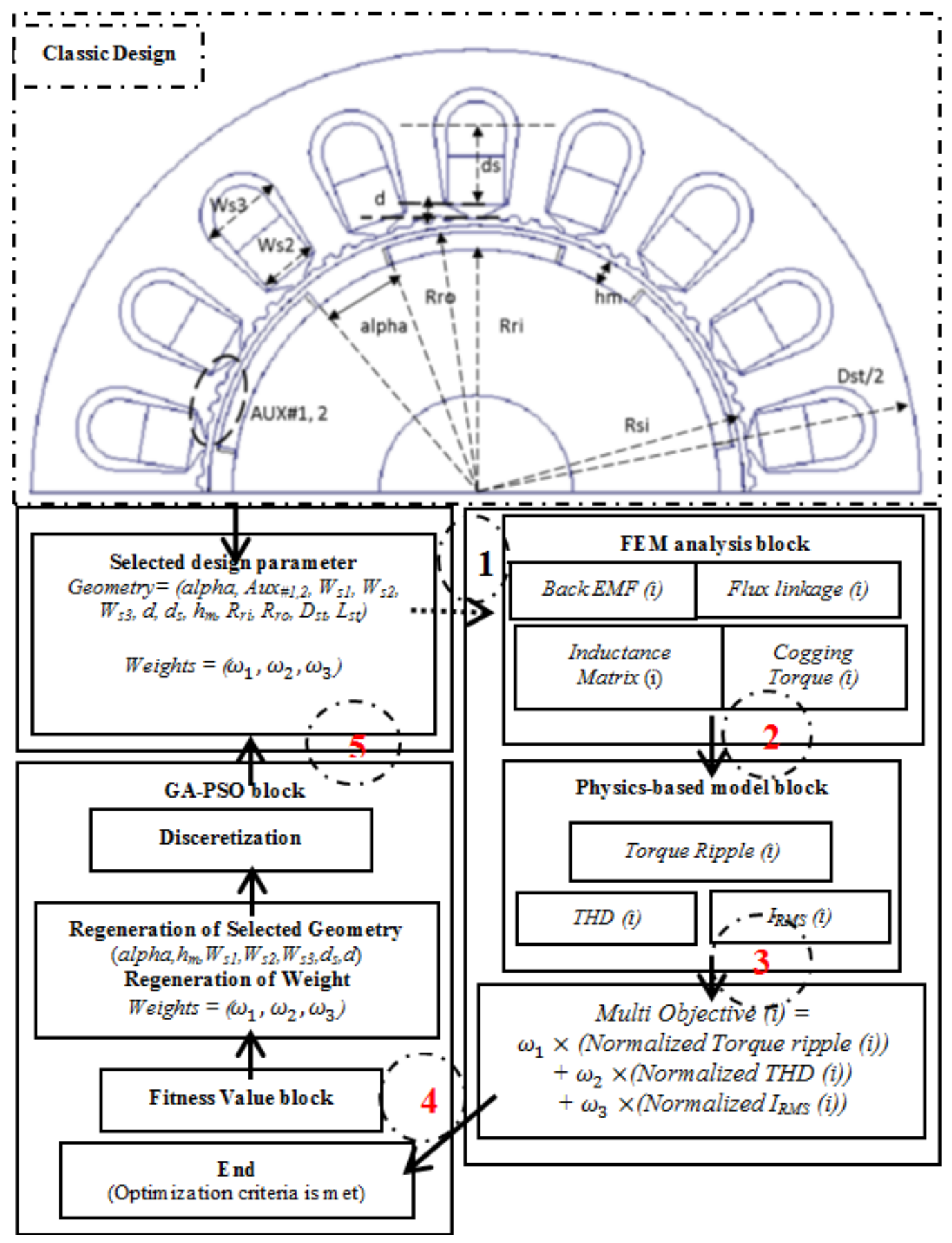

Figure 7.2 Schematic view of the multi-objective field-performance optimization

\subsubsection{Case of study}

A 36-slot, 2-hp IPM synchronous machine is designed based on the classic design process presented in chapter 2. The rated speed of the PM machine is $1200 \mathrm{rpm}$. This 
machine has a three phase and six magnet poles. The preferred magnet material is Samarium Cobalt, Sm2Co17, with remnant flux density of, $B_{r}, 1.08[\mathrm{~T}]$, with relative permeability, $\mu_{R}$, equal to 1.07 . Coercively of magnet, $H c$, equals to $900 \mathrm{KA} / \mathrm{m}$; the saturated flux density of the rotor and stator irons, $B_{\text {yoke }}$, equals to $1.25[\mathrm{~T}]$. The motor is designed for a current density equal to three $\mathrm{A} / \mathrm{mm}^{2}$, conductor space factor equals to 0.65 and average flux density of air gap equals to 0.56 [T]; the inter-pole angle equals to zero. The detail of the initial stator and rotor geometries are listed in Tables II, III and IV.

In this dissertation, it was assumed the as the pole width decrease, i.e. inter pole angle is increased, the pole height is increased proportional to such a change. Different relationship between pole height and pole width were tested and finally it was seen that the best relationship between these two can be stated as:

$$
h m_{1 \text { new }}=h m_{0}\left(1+\log \left(h m_{1 \text { pre }} / h m_{0}\right)\right)
$$

where $h m_{0}$ and $h m_{1 \text { pre }}$ are calculated as:

$$
\begin{aligned}
& h m_{0}=R_{r o}-\sqrt{R_{r o}^{2}-\left(P . A_{\operatorname{mag}} / \pi\right)} \\
& h m_{1 \text { pre }}=R_{r o}-\sqrt{R_{r o}^{2}-\left(A_{\text {mag }} /\left(\frac{\pi}{P}-\frac{\pi \cdot \alpha}{360}\right)\right)}
\end{aligned}
$$

In above equations and as shown infigure $7.2, A_{m a g}$, is the initial designed area of magnet, $R_{r o}$ is the outer diameter of rotor, $P$ is the number of poles and $\alpha$ is the interpole angle in degree.

In the optimization process, the candidate design parameters of the PM machine are the pole heights, $h_{m}$, stack length, $L_{s t}$ and inter-pole angles, $\alpha$, which basically 
change the magnet poles volume and lower slot width $W_{s 2}$, upper slot width $W_{s 3}$ and slot height $d_{s}$ which basically change the copper volume in each slot. The auxiliary teeth, Aux. \#1 and Aux. \#2, are placed for having more flexibility in the design, see table I. It should be mentioned that the variables can change the magnets and the copper areas. Consequently, the Ohmic losses and cost of machine can be changed although in this work these objectives were not considered directly.

The outer radius of the rotor and stator are not the needed design parameters. A series of floating points, which adapt the design parameters of machine are used as the input variables of the optimization problem. Moreover, the machine geometries are allowed to change in two directions, i.e. in radial direction and in the axial direction. Here, the relationships between the dimensions change in the stack length direction and in the radius direction are expressed as:

$$
\begin{aligned}
& L_{s t}^{\text {new }}=C_{f} \cdot L_{s t}^{\text {old }} \\
& R^{\text {new }}=R^{\text {old }} / \sqrt{C_{f}}
\end{aligned}
$$

Table 7.1 Boundaries of optimization input variables

\begin{tabular}{|c|c|c|c|c|c|}
\hline $\begin{array}{c}\text { Radial height } \\
\text { of Aux.\#1 } \\
(\mathrm{mm})\end{array}$ & $\begin{array}{c}\text { Radial } \\
\text { height of }\end{array}$ & $\begin{array}{c}\text { Inter-pole } \\
\text { angle } \\
\text { Aux.\#2 } \\
(\mathrm{mm})\end{array}$ & $\begin{array}{c}\text { Pole height } \\
\left(h_{m}\right)\end{array}$ & $B_{\text {yoke }}[\mathrm{T}]$ & \\
\hline$[0,0.5]$ & {$[0,2.4]$} & {$[0,10]$} & {$[2.8,4.5]$} & {$[0,1.3]$} & {$[0.9,1.1]$} \\
\hline$W_{s 2}(\mathrm{~mm})$ & $W_{s 3}(\mathrm{~mm})$ & $d_{s}(\mathrm{~mm})$ & $\left.J\left(\mathrm{~A} / \mathrm{mm}^{2}\right)\right)$ & $L_{s t}(\mathrm{~mm})$ & $\bar{T}_{e}(\mathrm{~N} . \mathrm{m})$ \\
\hline$[5.5-6.5]$ & {$[7-8.5]$} & {$[18-20]$} & {$[2,5]$} & {$[65-85]$} & 11 \\
\hline
\end{tabular}

In the optimization process, for the calculation of the fitness value, two random numbers are generated between zero and one. Based upon those two random numbers, the three weights for torque ripple, THD and RMS value of phase currents 
are calculated as:

$$
\omega_{1}=\frac{u_{1}}{u_{1}+u_{2}}, \omega_{2}=\frac{1-u_{1}}{1+u_{2}}, \omega_{3}=\frac{u_{2}}{1+u_{2}}
$$

Step 2.3: Calculate the fitness value as:

$$
f\left(X_{i}\right)=\sum_{k=1}^{3} \omega_{k} \cdot Y_{k} \cdot\left(X_{i}\right)+\operatorname{penalti}\left(J_{\max }\right)+\operatorname{penalti}\left(B_{\max }\right)
$$

where the penalties are defined as:

$$
\begin{aligned}
& \operatorname{penalti}\left(J_{\max }\right)=3 \times\left[\left(I_{R M S}(A) / \text { Conductor area }\left(\mathrm{mm}^{2}\right)\right)-5\right]^{3} \\
& \operatorname{penalti}\left(B_{\max }\right)=\left[B_{\max }[T]-1.3\right]^{5}
\end{aligned}
$$

Here, the constraint of the $B_{\max }$ was calculated when the stator is exited, however the field in a PM machines is mostly dominated by the magnets rather than the armature coils.

The parameters $Y_{1}$ through $Y_{3}$ are normalized variances of torque ripple, equation (7-17). They are also normalized to total harmonic distortion of the phase current, equation (7-18), the RMS value of the phase current, the denominator of equation (718), penalti $\left(J_{\max }\right)$ is the penalty factor of maximum allowable current density and penalti $\left(B_{\max }\right)$ is the penalty factor of maximum allowable flux density, respectively. In this work, the motor constant function, equation (7-19), as an indication of the cost of producing the average torque is served as a criterion for comparison between the initial and optimal design.

$$
\begin{aligned}
& \operatorname{Variance}(T)=\sum_{i=1}^{m}\left(T_{e i}-\bar{T}_{e}\right)^{2}, m=1,2, \ldots, \frac{t_{\text {stop }}}{t_{\text {step }}}+1 \\
& T H D=\frac{I_{H}}{I_{R M S}}=\frac{\sqrt{I_{3}^{2}+I_{5}^{2}+\ldots+I_{2 n-1}^{2}}}{\sqrt{I_{1}^{2}+I_{3}^{2}+\ldots+I_{2 n-1}^{2}}}
\end{aligned}
$$




$$
K_{m}=\frac{\left(T_{e} / 3\right)}{\sqrt{R_{a} \cdot I_{R M S}^{2}}}
$$

In equations (7-14) through (7-19), $T_{e i}$ is the instantaneous torque, $\bar{T}_{e}$, is the

average toque, $I_{R M S}$, is the RMS value of current in phase A and THD is the total harmonic distortion of current. Here ' $m$ ' in equation (7-7) is fixed to ten. The whole optimization process is shown infigure 7.2. In this study, mutation probabilities for the iteration intervals of [5-10] are five percent. Following several simulations, it was observed that the algorithm converges for up to six iterations. Consequently, the maximum number of iterations is set to 10 . Various simulations show that the algorithm is converged after 130000 seconds. The consumed CPU and memory were $1.17 \mathrm{~GB}$ and $3 \mathrm{GHz}$, respectively.

\subsubsection{Simulation results}

Figure 7.3 shows a comparison between initial and optimized back EMF waveforms, it is seen that the optimized machine prefer to have a more smooth back EMF waveform specially on the top of waveform. The back EMF of the original motor is the summation of a trapezoidal waveform and a dominant sine wave. The frequency that dominant the sine wave is five times of the fundamental frequency of the back EMF waveform. The back EMF waveform of the optimized machine is a semi-sinusoidal waveform with improved area and a dominant half cycle sine waves with respective frequencies nine times the fundamental frequency of the semisinusoidal back EMF waveform.

Figure 7.4 shows a comparison between the cogging torques before and after optimization. The optimized machine has a cogging torque at least four times lower than the cogging torque of the initial design and the frequency of the cogging torque 
is increased twice.

Figure 7.5 shows the self and mutual inductances before and after optimization. As can be seen from this picture, the absolute amplitude values, of both self and mutual inductances, are increased. This is more desirable for the current dive.

Figure 7.6 shows the flux linkage of the machine before and after optimization. It is seen that the maximum value of the flux linkage has increased. This will help the machine to be supplied by a lower current.

Figure 7.7 shows the total torque of the machines before and after optimization. As illustrated in this figure, the torques ripple of the optimized machine was decreased at least four times in comparison with the torque ripple of the initial design. A part of this ripple reduction is because of the reduced cogging torque and a part of that is because of the minimization of the mismatching between the current and back EMF waveforms. The comparison between the phase of self and mutual inductances in one phase before and after optimization show that after optimization, a phase shift has occurred in the inductances that mainly will cause a developed reluctance torque in the machine. This is due to the change of the rotor shape and its material in the vicinity of the air gap after the optimization.

Figure 7.8.a shows the simulated speed of the original and optimized machines. From figure 7.8.b, it is observed that the optimized machine has a small overshoot at start up. The enlarged view of the speed shows that that speed ripple of the optimized machine has reduced, although this was not a direct objective. 


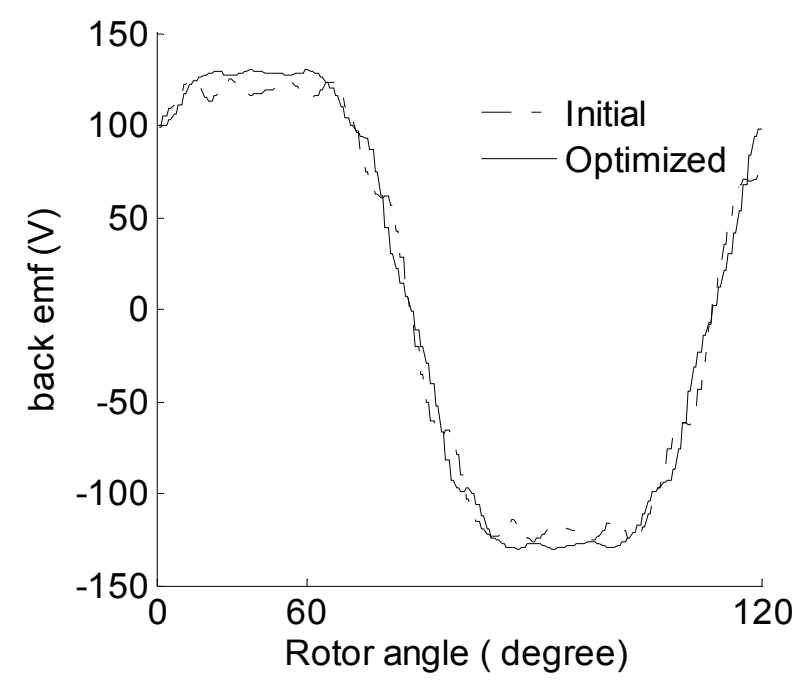

Figure 7.3 Back EMF of the phase A before and after optimization

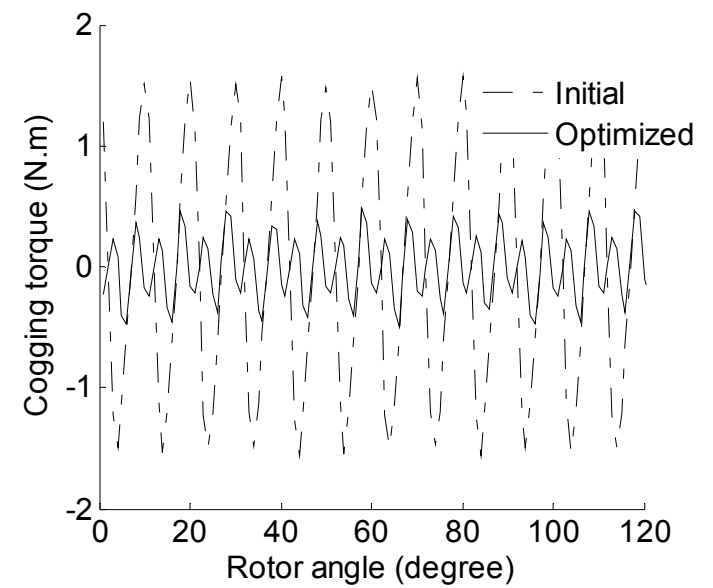

Figure 7.4 The cogging torque in initial and optimized design

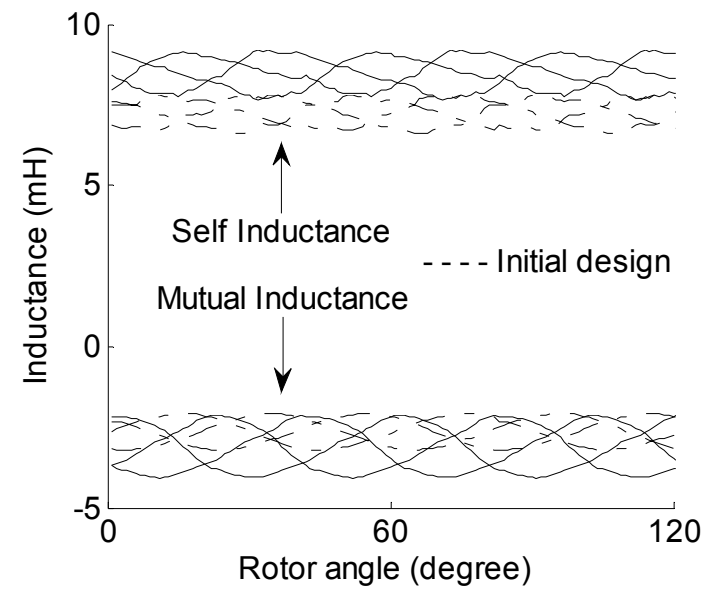

Figure 7.5 Self and mutual inductances before and after optimization 


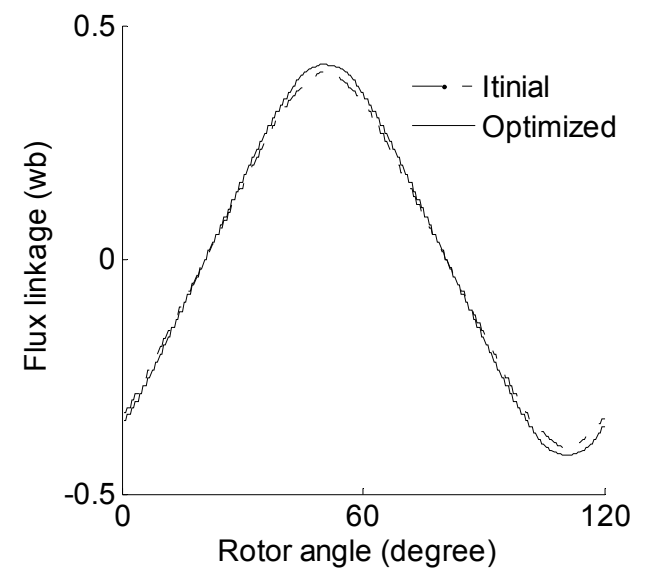

Figure 7.6 Flux linkage of the phase A before and after optimization

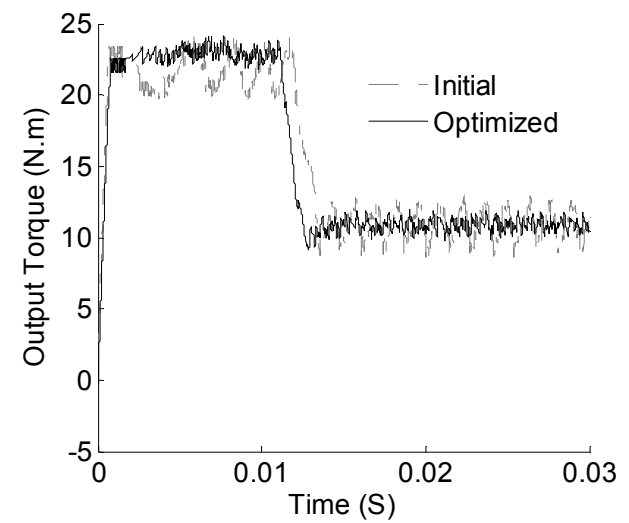

Figure 7.7 Total torque before and after optimization
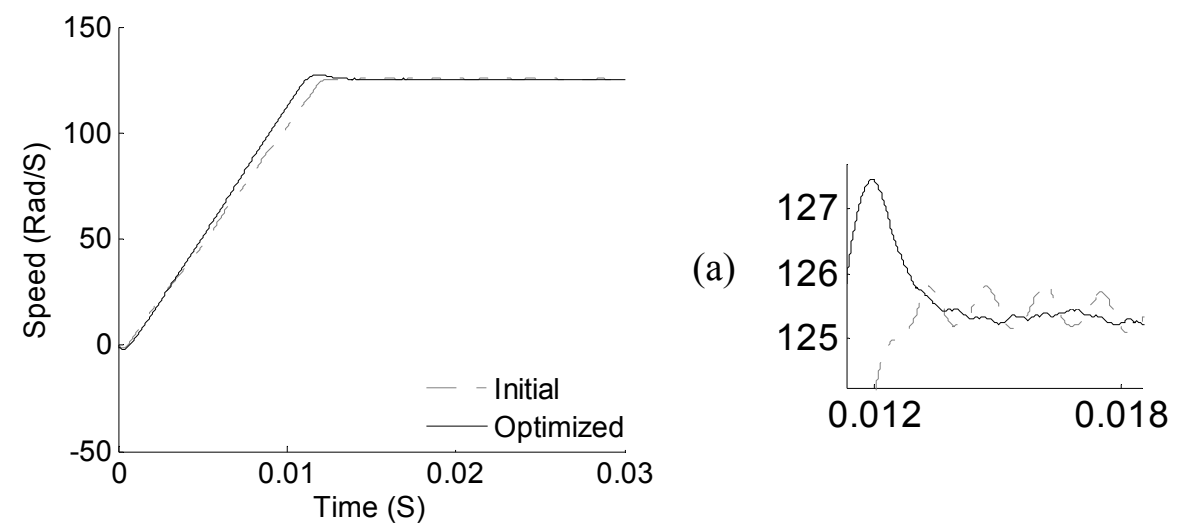

(b)

Figure 7.8 Speed before and after optimization

Tables 7.2, 7.3 and 7.4 show the initial and the optimal geometries of the machine. As can be concluded from these Tables, there is no need for Aux\#1. The reason is 
that, this tooth leads to iron saturation in the teeth opening, whereas Aux\#2 which manly influence the back EMF waveform as well as cogging torque is properly shaped. Moreover, the volume of copper and magnets before and after optimization are shown in table 7.5. It is seen that the copper and magnet volume of the optimized machine are reduced. In fact the reduced root mean squared value of current leads to reduction of the copper area.

Table 7.6 shows a comparison between the performance measures of the machine before and after optimization. As can be seen, almost all of the desired goals were achieved.

\subsubsection{Discussion}

By a comparison of the phase current and the back EMF waveforms of the initial and the optimized designs, figures 7.9.a and 7.9.b, it can be observed that, the current of the optimized machine is visually closer to its back EMF. Moreover, by a visual comparison of the current waveform and the back EMF waveforms in the vicinity of the peak of the back EMF, see figures 7.10.a and 7.10.b, it is observed that the current is better matched to the back EMF in the optimized machine. In fact, the vicinities of the peak of the back EMF are where the maximum energy conversions occur.

Figure 7.11.a and 7.11.b show the phase current waveform of the initial and the optimized machines. As can be seen from this figure, the high order harmonic contents of the initial machine is more than the high order harmonic contents of the optimized machine. One can say that the low high order current harmonics will result in lower magnetic loss in the iron cores, although the magnetic loss was not a direct objective. Furthermore, as mentioned in the classic design procedure, the current

density is assumed (3) three $\left(A / \mathrm{mm}^{2}\right)$, However, as illustrated in table 6 , the current 
density of the initial machine is calculated as $4.84\left(\mathrm{~A} / \mathrm{mm}^{2}\right)$. In fact, the classic design procedure will not provide the designer with accurate information about the machine unless the dynamical physics-based model of the machine is calculated and simulated. Here, the current density was calculated from the division of the phase current calculated from the physics-based model and the conductor area in each of the slots.

This work was conducted with some of the geometrical design parameters of the machine were introduced as optimization variables and the current drive circuit was seen as a black box. Indeed, this is helpful where the machine manufactures need to make the design of their machines compatible to an existing drive in the market. This process is feasible with a real-time hardware in loop (HIL) optimization process. From another point of view, for a complete optimization of the motor-drive system both of motor and drive can be optimized together. In this view, a low frequency physics-based model of motor-drive systems will be needed.

Table 7.2 Stator dimension before and after optimization $(\mathrm{mm})$

\begin{tabular}{|c|c|c|c|c|c|}
\hline Stator dimension & $D_{s t}$ & $d_{s}$ & $d$ & $W_{s 2}$ & $W_{s 3}$ \\
\hline Initial & 119.2 & 19.82 & 1.9 & 6.4 & 8.1 \\
\hline Optimized & 117.9 & 18.9 & 1.8 & 6.1 & 7.7 \\
\hline
\end{tabular}

Table 7.3 Stator dimension before and after optimization ( $\mathrm{mm})$

\begin{tabular}{|c|c|c|c|c|c|}
\hline Stator dimension & $R_{r i}$ & Stack length $\left(L_{s t}\right)$ & Aux\#1 & Aux\#2 & Conductor/slot/phase \\
\hline Initial & 66.54 & 74.5 & 0 & 0 & 17 \\
\hline Optimized & 6.34 & 81 & 0 & 2 & 17 \\
\hline
\end{tabular}

Table 7.4 Rotor dimension 6 pole, $2 \mathrm{hp}$, SPM machine (mm)

\begin{tabular}{|c|c|c|c|c|c|c|}
\hline Rotor Dimension & $R_{r i}$ & $h_{m}$ & $S_{R}$ & $g$ & $R_{r o}$ & $\alpha$ (degree) \\
\hline Initial & 63.5 & 3 & 14.8 & 3.2 & 66.5 & 0 \\
\hline Optimized & 63.4 & 3.1 & 14.8 & 3.2 & 66.5 & 3.5 \\
\hline
\end{tabular}


Table 7.5 Volumes and mass before and after optimization

\begin{tabular}{|c|c|c|c|}
\hline Machine & $\begin{array}{c}\text { Copper volume } \\
\text { in each slot }\left(\mathrm{cm}^{3}\right)\end{array}$ & $\begin{array}{c}\text { Each Magnet } \\
\text { pole }\left(\mathrm{cm}^{3}\right)\end{array}$ & Mass(kg) \\
\hline Original & 16.16 & 15.42 & 7.43 \\
\hline Optimized & 15.95 & 14.98 & 8.18 \\
\hline Saved & 0.21 & 0.44 & 0.75 \\
\hline
\end{tabular}

Table 7.6 Machine performance before and after optimization

\begin{tabular}{|c|c|c|c|c|c|c|}
\hline Performances & THD (\%) & $\mathrm{km}$ & $I_{R M S}$ & $\operatorname{Var}(\omega)$ & $R_{a}(\Omega)$ & $J\left(A / \mathrm{mm}^{2}\right)$ \\
\hline Initial & 5.1 & 0.889 & 4.61 & 3.5 & 0.807 & 4.84 \\
\hline Optimum & 4.1 & 0.848 & 4.37 & 2.7 & 0.977 & 4.66 \\
\hline
\end{tabular}
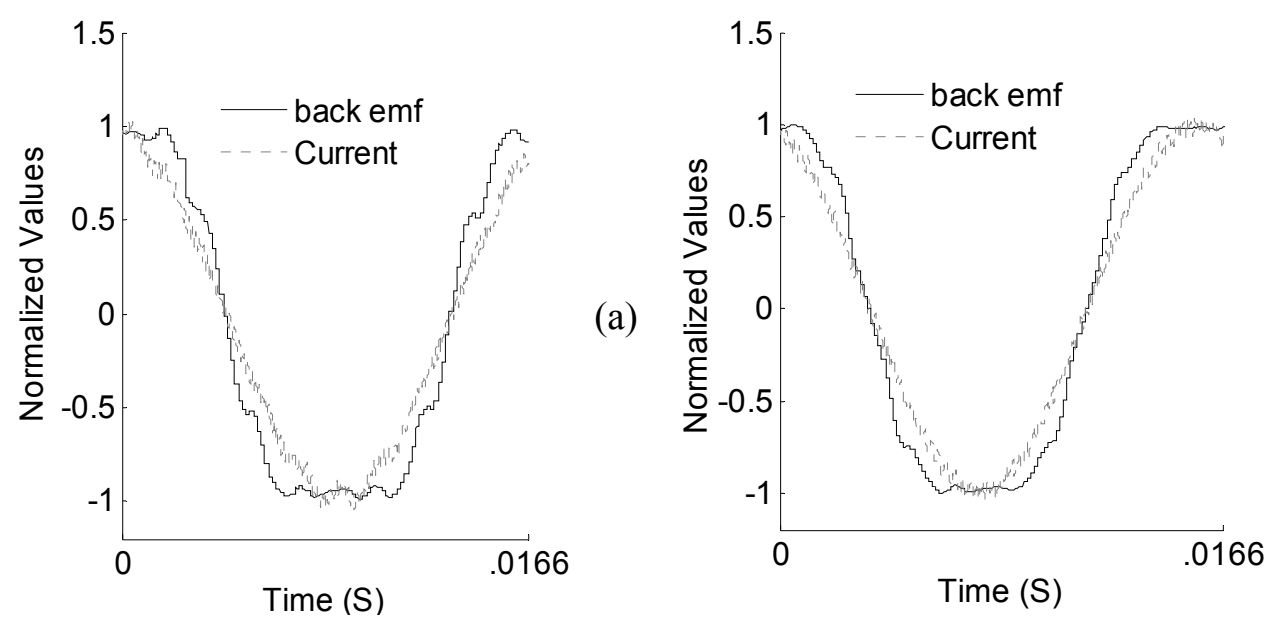

Figure 7.9 The current-back EMF waveforms before (a) and after optimization (b)

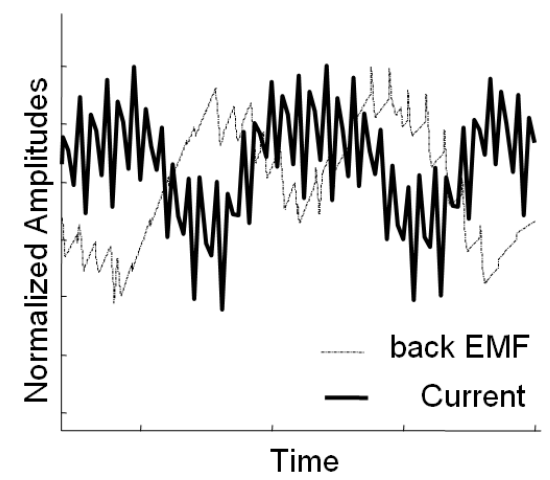

(a)

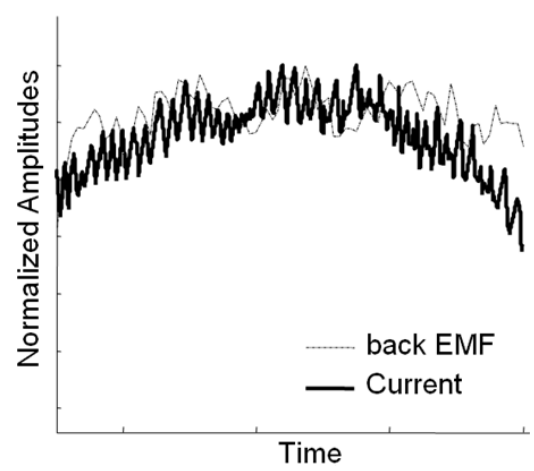

Figure 7.10 The current-back EMF waveforms at the vicinity of the peak of the back EMF before (a) and after optimization (b) 


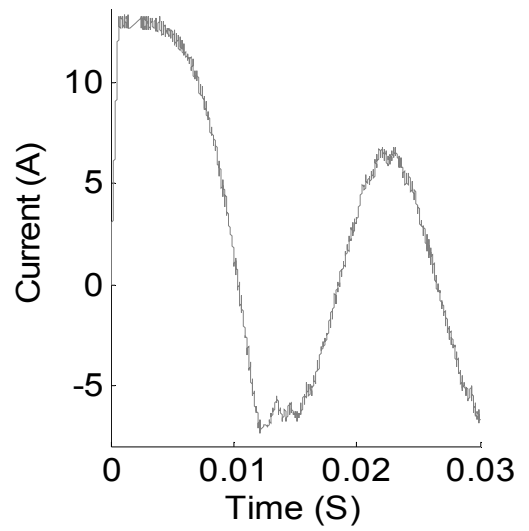

(a)

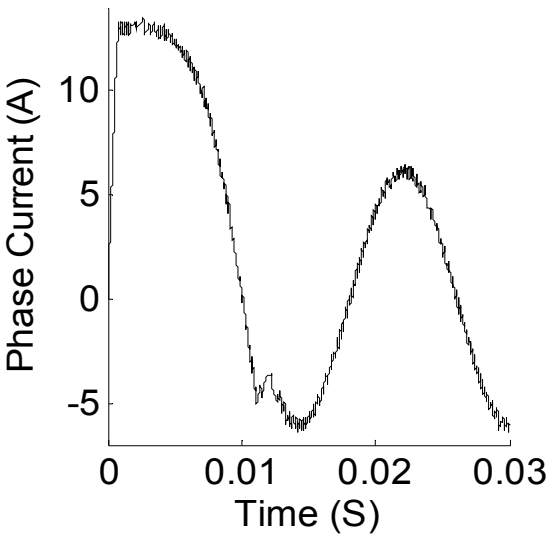

(b)

Figure 7.11 The Current waveforms before (a) and after optimization (b)

\subsection{Hardware in the loop based finite element design optimization process for the computational prototyping of electric motor-drives}

The design optimization of PM machines can be purely done in an FE-based design optimization process neglecting the dynamic effect of the drive or at least utilizing the effect of a typical drive inside a full software environment. Following the design of the machine, the proper choice of the drive could be made concerning different limitations and requirements imposed from the environment, application or machine. Basically, the selection of the right drive consists of several steps including:

- Selecting the right model based upon the motor's horse power rating, motor voltage, motor full load current and control modes.

- Selecting the proper rating based upon the application. For example in a typical application a K-hp motor can be controlled by K-hp drive, but there are some nontypical applications which dictate the size of the drive with respect to the size of the machine. Temporary overload requirement of the drive is also one of the factors that affect the choice of a drive.

- The accessories of electric drives such as EMI filters, dynamic breaking resistors and line reactors can also be viewed as part of drives that affect the performance 
and working conditions of electric machine-drive system.

The separate design of machine and selection of its drive is a conventional process; however, incompatibility of the machine and the drive may affect the performance of the machine. For example, the choice of a specific drive for a machine from conventional way may satisfy the steady state or even transient conditions of a machine-drive system for all operational contingencies however, it may not be lead to an optimum operation. For instance, the different modulation techniques, filtering, or speed controller bandwidth in the drive create different voltage or current harmonics in the machine. The associated voltage and current harmonic with the machine will not always be the source of unwanted effects such as loss, vibration, torque and speed ripples. However, it will be torque producer if well matched with the machine's physical characteristics and EMF harmonics. From this prospective, the coupled design compatibility optimization of an electrical machine with a pre-chosen drive will offer more advantage than separate design of machine and drive. Coupled design compatibility optimization of the electrical machine and the drive can be done via either numerical or analytical modeling of the machine and drive and their accessories. If the machine designer has less information about the the drive package, inaccurate and unreliable results may be achieved. From this point of view, the selection of the real drive based on the basic rating requirement of the desired machine and then the design of the machine in the hardware in loop (HIL) optimization process will be a possible solution. In this dissertation, HIL-FE based technique for the design optimization of a PM motor with an existing currentregulated AC drive is proposed. The comparisons of the calculated results before and after the optimization procedures show the performance improvements as well as material savings. In order to create the HIL-FE optimization process using the 
following steps:

Step1-The equivalent physical hardware model of the machine that can vary with the design change is needed. The equivalent physical hardware of the machine during optimization is a current adjustable load (CAL). This includes a software part and a hardware part. The software part is a physics-based model of the machine which was explained earlier in chapter 3 . The hardware part is experimentally created by a buck and a fully controlled 3-phase converter.

Step2-A population based optimization process is needed. The design objectives are set to explore the best trade-offs between the geometrical design parameters of the PM machine for minimum torque ripple, minimum RMS value of phase current, a minimum total harmonic distortion (THD) of phase current and the desired torque versus speed profile [98]. These single objectives together with the maximum drive current, magnetic saturation constraints and the thermal limitation, which is imposed via current density, are set to be satisfied at the same time in a multi-objective function. These objectives were simultaneously calculated from the actual phase current of the CAL connected to the real drive during the design optimization process. The drive is seen as black box hardware.

\subsubsection{The hardware in loop compatible physics-based modeling of the PM machine}

In order to control the equivalent hardware of the machine, a fast model of the machine compatible to hardware interface is required. The physics-based model of a PM machines presented in chapter 3 is an accurate, fast and non-linear dynamic model for the purpose of HIL integrated drive system simulations. In comparison with the d-q and the full FE-based models, the physics-based model has the accuracy very close to a full FE-based model and it has a simulation run time close to the simple d-q 
model. This model uses non-linear transient finite elements (FE) solutions to establish a detailed block description of the implemented machines in a Simulink-MATLAB environment. This modeling method can be equipped with flux weakening, dynamic core losses and saturation as explained in chapter 3. This model is essentially a database representation of the non-linear transient operation of the machine. The physics-based model can be simply and quickly recreated when the machine geometry and material is changing during an optimization process.

\subsubsection{HIL-FE based optimization Process}

\subsubsection{Soft multi-objective optimization}

As discussed, in the design of the PM machines, it is possible to adjust the back EMF waveform by changing the geometrical design parameters of the machine or changing the materials properties such as the magnetization of the magnets. Alternatively, it is accepted that the established phase current waveform through a controlled PMSM machine is generally proportional to the back EMF waveform. Therefore in the form of an inverse problem it is possible to change the back EMF waveform for having a more desirable current waveform. Similar to the back EMF waveform, to some extent, cogging torque also affects the current waveforms. For example, for having a ripple free machine, the developed torque as the interaction of back EMF and phase current plus the reluctance torque should actively cancel the cogging torque. If we assume that we have no control over the back EMF and the inductance waveforms. This is since they depend on the physical features of the machine. The only way to have a control over the developed torque is definitely through phase current. Consequently, it can be mentioned that, the cogging torque also affects the waveform of the phase current.

The phase current, firstly, affects the torque ripple of the machine; secondly, it 
affects the total harmonic distortion of machine and thirdly, it affects the active losses.

On the other hand, the torque ripple of a PM machine originate from several sources i.e. the cogging torque, the mismatching between the current waveform and the back EMF waveforms, iron saturation and the mismatching between the phase inductances and the phase currents. In general, there are three possible methods to reduce the torque ripple. The first method is to design the drive of the machine with respect to the maximum compatibility of the phase current to the back EMF of the machine. The second method is to design the geometry and material of the machine for the minimum cogging torque. Finally, the third method is to determine the geometrical design parameters of the machine with respect to the minimum cogging torque and the most compatible back EMF waveform to the phase current. In addition, it is evident that the drive switching frequencies, modulation technique, drive topology and current and speed control loops are the basic elements that affect the phase current and therefore the torque and speed profiles.

From machine design point of view, the machine's back EMF waveform is mainly influenced by magnetic materials characteristic such as magnets magnetization (radial or axial), magnets geometries and magnets location in the rotor and magnets temperature. On the other hand, in geometrical solution category where the cogging torques originates from the energy variation of the air gap field due to the mutual interaction of the rotor magnets and stator slot openings and the teeth. There are various techniques to reduce the cogging torque. Basically the main principles underlying reduction of the cogging torque is to reduce abruptness of pole-teeth attraction which can be performed by magnet reshaping as well as teeth and pole skewing. Moreover, one of the main objectives for improving the torque versus speed profile is through reducing the characteristic current of the machine. This is feasible 
by increasing the reluctance torque either by a proper PM design and/or the creation of an unbalanced air gap and reshaping of the slot opening. Therefore the goals of optimization are summarized as:

Minimum distance between calculated and a predefined torque-speed profile , equation (7-28), Minimum torque ripples at five arbitrary different points of torque speed profile, equation (7-24), Minimum THD of phase current at five arbitrary different points of torque speed profile, equation (7-26).

Constraints of optimization are defined as:

- Maximum available DC bus voltage, equation (7-23)

- Maximum allowable current density equation (7-21)

- Maximum allowable flux density in no load condition, equation (7-22)

- Maximum current provided by the drive, equation (7-28)

- Predefined speed setting time, equation (7-28)

All of the optimization goals and constraints must be satisfied in a single classic multi-objective function. The optimization algorithms is a population based process that employs both particle swarm and genetic algorithm operators. The details of this algorithm are presented at sections 7.3. Here, the fitness value, $\mathrm{f}\left(\mathrm{x}_{\mathrm{i}}\right)$, is chosen as:

$$
\begin{aligned}
& f\left(x_{i}\right)=\sum_{m=1}^{M} \omega_{m} \cdot Y_{m} \cdot\left(X_{i}\right)+\operatorname{penalti}\left(J_{\text {max }}\right)+\operatorname{penalti}\left(B_{\text {max }}\right)+\operatorname{Limit}\left(E M F_{\text {max }}\right) \\
& \operatorname{penalti}\left(J_{\text {max }}\right)=K_{J \max } \times\left[\left(I_{R M S i}(A) / \text { Conductor Area }\left(\mathrm{mm}^{2}\right)\right)-2\right]^{n 0} \\
& \operatorname{penalti}\left(B_{\text {max }}\right)=\left[B_{\text {max }_{i}}[T]-1.3\right]^{n 1} \\
& L\left(E M F_{\text {maxi }}\right)=\left(E M F_{\text {maxi }} \mid E M F_{\text {maxi }} \leq\left(V_{d c} / 2\right)\right)
\end{aligned}
$$

where $Y_{m}^{\prime}\left(X_{i}\right)$ is the normalized objective function of the $Y_{m}\left(X_{i}\right)$, " $m$ " is the index 
of objectives, " $M$ " is the number of objectives and $\omega_{m}$ is the weight and $\sum_{m=1}^{M} \omega_{m}=1$.

It follows that $Y_{m}^{\prime}\left(X_{i}\right)$ are chosen as the variance of an average torque ripple, equations (7-24), the total harmonic distortion of phase current, equation (7-26), the RMS value of the phase current and average of motor power for a set of torque versus speed. The value $Y_{m}^{\prime}\left(X_{i}\right)$ should be normalized before applying it to equation (7-20). Here, the parameters $n^{0}, n^{1}, K_{B \max }$ and $K_{J \max }$ control the penalty factors. " penalti $\left(J_{\max }\right)$ " is the penalty factor of maximum allowable current density of stator conductor in each phase for the machine design number " $i$ " and penalti( $\left.B_{\max i}\right)$ is the penalty factor of the maximum allowable flux density in the back iron, stator teeth and rotor inter-pole angle, $I_{R M S_{i}}$ is the root mean square (RMS) value of the phase current, "ConductorArea" is the area of copper. The term "Limit $\left(E M F_{\text {max }}\right)$ " is the maximum allowable EMF for nominal speed in the machine design number " $i$ ". For a radial flux Y-connected surface mount PM motor without access to neutral, if the weak flux weakening is neglected, the maximum allowable back EMF for maximum speed, should be chosen not to exceed the maximum phase to neutral voltage of the drive to ensure a proper speed control by a slow PI controller with relatively small proportional gain, $K_{P}$ and large integrator gain, $K_{I}$. In this work, the maximum allowable back EMF, $\operatorname{Limit}\left(E M F_{\text {max }}\right)$, for a nominal speed is chosen as the half of the maximum value of the DC bus voltage, $V_{d c}$, of the drive. This is because of the fact that the maximum amplitude of the fundamental component of the phase to neutral voltage for the used three phase fully controlled AC drive is half of the DC voltage. This is chosen based on the fact that the maximum fundamental component 
of the phase to neutral voltage of the fully controlled converter is directly related with the modulations techniques. For example 180 controlled AC drives voltage source operation, PWM, third harmonic injection, space vector and hysteresis can be $\left(2 V_{d c} / \pi\right),\left(V_{d c} / 2\right),\left(V_{d c} / \sqrt{3}\right),\left(V_{d c} / \sqrt{3}\right)$ and $\left(V_{d c} / \sqrt{3}\right)$ respectively[83]. Evidently, if the drive modulation technique is unknown, the statement, $0<\operatorname{Limit}\left(E M F_{\max i}\right) \leq K .\left(V_{d c} / 2\right)$, should be satisfied during optimization process. In this statement, " $K$ " can be chosen as the optimization variable with the boundaries of $1 \leq K \leq \frac{4}{\pi}$. Here, $V_{d c}$ is the available DC bus voltage. In the final results, the motor constant function, equation (7-27), was used as an indication of the cost of producing the average torque and is served as a criterion for comparison between the initial and optimal design at different points of the torque-speed profile.

$$
\operatorname{Variance}\left(T_{i}\right)=0.2 \times \sum_{j=1}^{5}\left(\sum_{e=K / 2}^{K}\left(T_{i j e}-\bar{T}_{i j}\right)^{2}\right)
$$

$$
\begin{aligned}
& \bar{T}_{i j}=\operatorname{mean}\left(\bar{T}_{i j e}\right)_{e=K / 2}^{K}, K=\frac{t_{\text {stop }}}{t_{\text {step }}}+1 \\
& T H D_{i j}=\frac{I_{H_{i j}}}{I_{R M S_{i j}}}=\frac{\sqrt{I_{3_{i j}}^{2}+I_{5_{i j}}^{2}+\ldots+I_{(2 n-1)_{i j}}^{2}}}{\sqrt{I_{1_{i j}}^{2}+I_{3_{i j}}^{2}+\ldots+I_{(2 n-1)_{i j}}^{2}}} \\
& K_{m_{i j}}=\frac{\left(\bar{T}_{i j} / 3\right)}{\sqrt{R_{a} \cdot I_{R M S i j}^{2}}}
\end{aligned}
$$

where " $i$ ", " $j$ " and " $e$ " are the number of under optimization machine, number of operating points in torque-speed profile and the number of sample data existing from the torque profiles, respectively. 
In equations (7-24) to (7-27), $T_{i j}$ is the instantaneous electrical torque, $\bar{T}_{i j}$, is the average of instantaneous torque, $\mathrm{t}_{\text {stop }}$ is the stop time for one simulation, $t_{\text {step }}$ is the stet time for one simulation, index " $j$ " denotes a point in torque-speed profile, index " $n$ " denotes a harmonic order, $R_{a}$ is the phase resistance and $K_{m_{i j}}$ is the motor constant.

\subsubsection{Calculation of torque vs. speed}

The mentioned objectives in equations (7-24) to (7-26) were calculated with respect to different points of torque vs. speed map. The torque vs. speed map was calculated from HIL-FE based model based on the basis that each point in the torquespeed map should satisfy the stability of the system, the maximum allowable current of the drive and the maximum current density of the machine with respect to temperature limitations. The mathematical description for calculation of this map is summarized as:

$$
\begin{aligned}
\left\{\left(T_{0}, \omega_{0}\right) \mid\left(I_{\text {phase }}(t)<I_{\max }\right) \cap\left(I_{\text {phase }}(t)<J_{\max } \times A_{\text {conductor }}\right)\right. \\
\left.\cap\left(\left|T-T_{\text {ref. }}\right|<\varepsilon\right) \cap\left|\omega_{m}-\omega_{\text {ref. }}\right|<\varepsilon_{1} \forall 5 t_{s}<t<10 t_{s}\right\}
\end{aligned}
$$

In above statement, $\mathrm{T}_{0}$ and $\omega_{\mathrm{f}}$ are the desirable reference torque and speed. Also, $I_{\text {phase }}$ is the phase current, $\mathrm{I}_{\max }$ is the drive full load currents, $\mathrm{J}_{\max }$ is the maximum current density of the machine and $A_{\text {conductor }}$ is the area of the conductor passing $I_{\text {phase }}$. The value of $J_{\max } \times A_{\text {conductor }}$ is set based on thermal limitations of the machine. Here, $\mathcal{E}$ and $\varepsilon_{1}$ are the torque and speed tolerance. $\omega_{m}$ is the measured angular velocity, $T$ is the torque, $t$ is the time and $t_{s}$ is a desirable setting time for the speed.

\subsubsection{Setting of the current adjustable load (CAL)}

The hardware-in-the-loop (HIL) technique is very useful for machine control 
studies as a computational prototyping method. In normal practice, the actual machine is used in HIL real time simulation systems to examine the controller realized by a software module[201]. Furthermore, based on the discussion presented in reference[197], the fully automated computational design environment needs to iteratively change the design parameters in the optimization process to satisfy the objective function. The developed optimization process can automatically connect to the numerical FE domain, simulink domain and it is capable of being employed in a HIL process. This is since the fast FE-base phase variable model [45]for the machine, calculated in each iteration of the optimization, can be automatically built in a hardware based interface, which includes the dSPACE1103, to run the machine with actual drive system. The current adjustable load simulates the equivalent hardware of the machine during the design procedure. It follows the reference phase current created in the software domain by the FE based phase variable model. The current adjustable load was created from a three phase resistive-inductive (R-L) line in series with the three phase fully controlled converter. The output of the converter is connected to a buck converter as shown in figure 7.12. Because the optimization is an iterative process that searches for hundreds of possible solutions of the machine, the current adjustable load should have enough flexibility to respond to design variations. If the mutual inductances of each motor design are neglected and it is assumed that the resistance and inductance of the R-L line is equal to the resistance and inductance of the machine, then the input voltage to three phases fully controlled converter should be equal to the back EMF voltage of the machine. In order to do this, the buck converter should be designed and controlled to create a variable DC voltage, $V_{\text {out }}$, controlled by the back EMF of the machine. It is assumed that for a given current 
command for the hysteresis control of the three phase converter, the peak line to neutral voltage on the AC side of the fully controlled converter is $V_{p k}$. Because the maximum line to neutral voltage that the bridge can supply is $2 V_{\text {out }} / 3$, it is apparent that $V_{p k}$ must be less than $2 V_{\text {out }} / 3$ if the commanded current is to be obtained. There is also another constraint that the peak line to line voltage $\sqrt{3} V_{p k}$ must be less than the peak line to line voltage the converter can achieve. This voltage is equal to $V_{\text {out }}$. This requirement is more restrictive and defines the steady state range over which we can expect the current to be tracked, in particular $V_{p k}<V_{\text {out }} / \sqrt{3}$. By restating this criterion, we will have $V_{\text {out }}>\sqrt{6}\left(V_{p k} / \sqrt{2}\right)$. If we assume that the RMS value of the line to neutral back EMF voltage, $\mathrm{V}_{\mathrm{EMF}}$ equals to $V_{p k} / \sqrt{2}$, then the minimum value of $V_{\text {out }}$ will ensure that a current control is calculated as $V_{\text {out }}>\sqrt{6} V_{E M F}$. Because $V_{E M F}$ cannot exceed the maximum of $\sqrt{2} V_{d c} / \pi$, therefore $V_{\text {out }} \geq 2 \sqrt{3} V_{d c} / \pi$. This means that the input of the buck converter should also be chosen as $V_{d c 1} \geq 2 \sqrt{3} V_{d c} / \pi$. On the other hand, a sinusoidal fully rectified back EMF has a DC value of $V_{\text {in }} \geq 2 \sqrt{2} V_{E M F} / \pi$. Now, if duty cycle of the buck converter is defined as $D=K V_{i n} / V_{d c 1}$ which is equal to $D=\sqrt{2} K V_{E M F} / \sqrt{3} V_{d c}$, in order to ensure that $\mathrm{Dcan}$ reach a maximum, one should always have $D=V_{\text {out }} / V_{\text {in }}=\left(\sqrt{6} K V_{E M F}\right) /\left(2 \sqrt{2} V_{E M F} / \pi\right)$. In order to ensure that " $D$ " will reach to one for the maximum possible back EMF, we should have $K \geq \pi \sqrt{3} / 2$. Here, it is chosen as $2 \sqrt{3}$ to also compensate the resistive and inductive voltage drops due to the phase resistance and inductance variations during the design optimization process. As discussed in the introduction, the role of optimization process is to modify the 
geometry or material for the better performance and not to form the design from scratch; as a result, the boundaries of geometries in optimization process have maximum $\pm 50 \%$ difference from the initial design, if the difference was more then there had been a serious mistake in the initial design procedure. In a HIL optimization process, the hardware set up is fixed during the optimization and therefore specification of a proper value for the RL line, $R_{a b c}$ and $L_{a b c}$, is necessary. Because the boundary of geometry in optimization process is limited, determination of the value of RL line from one of the random geometries using a static FE analysis is a solution. If we are interested to see the very fast transient phenomena, or high order harmonics of the machine, the value of inductance, $L_{a b c}$, play an essential role. An equal or lower value of the inductances $L_{a b c}$ from the machine's inductance does not omit the high order current harmonics while the higher $\mathrm{L}_{\mathrm{abc}}$ does. In theory, the best value of $L_{a b c}$ can be chosen as the difference of self and mutual inductances i.e. $L_{a b c}=L_{s}-M$

In a buck converter, the values of $L_{1}$ and $C_{1}$ were evaluated based upon an averaging value analysis. The calculated parameters of the CAL for the design process are collected in table 7.7. Experience shows that, fast hardware interface with high digital time step is required to ensure acceptable results. The sample time of the PWM carrier should at least be ten times less than the inverse switching frequency. Therefore, creation of the carrier PWM with higher time step independent from the main Simulink-MATLAB file is necessary to reduce the computational cost.

Table 7.7 Current adjustable load parameters

\begin{tabular}{|c|c|c|c|c|c|}
\hline$L_{1}(\mu H)$ & $C_{1}(\mu F)$ & $R_{a b c}(\Omega)$ & $L_{a b c}(m H)$ & $V_{d c}(V)$ & $V_{d c 1}(V)$ \\
\hline
\end{tabular}




\subsubsection{Case of study}

In order to start the design process, an existing surface mounted PM machine design which has relatively close performance to our desirable design was chosen. This initial design needs to be optimized for desirable performances. The initial design example is a $250-\mathrm{W}, 42-\mathrm{V}$ DC surface mounted PM synchronous machine, see Appendix I. The stator winding is three phase Y-connected. The stator has 12 slots. There are 4 coils per phase with 12 turns per coil. The rated speed and torque of the PM machine are 4000 (RPM) and 0.56 (N.m), respectively. This machine has ten magnet poles. The magnet material is a radial magnetized Samarium Cobalt, $\mathrm{Sm} 2 \mathrm{Co17}$, with remnant of magnets, $B_{r}$, equal to 1.08 Tesla, with relative permeability, $\mu_{R}$, equal to 1.07 .

The normal coercivity of magnet $H_{c}$, is around $900(\mathrm{kA} / \mathrm{m})$; the saturated flux density of the rotor and stator irons, $B_{\text {yoke }}$, equals to 1.25 Tesla. The motor is designed for a current density equal to1.5 $\left(\mathrm{A} / \mathrm{mm}^{2}\right)$, the average flux density of the air gap equals to 0.56 Tesla, the conductor space factor equals to 0.8 . The space factor was defined as the total area of copper to the total area of slot. The effective area of slot is $0.5 \times d \times\left(W_{s 2}+W_{s 3}\right)$. The inter-pole angle equals to zero. The windings are of the concentrated type.

The main candidate design parameters of the machine are listed in Table 7.8. The details of the initial stator and rotor geometries as well as the shape are listed in Tables 7.9, 7.10, 7.11 and figure 7.12. In the Table 7.9, At1and At2 are the height of auxiliary teeth, AUX is the circumferential width of auxiliary teeth, which are all 
equal. Here, $R_{r i}$ is the inner rotor radius, $R_{r o}$ is the outer rotor radius, $S_{R}$ is the shaft radius, $\mathrm{g}$ is the air gap length in radial direction, $L_{m}$ is the magnet length in radial direction and $\alpha$ is the inter-pole angle.

During the optimization process, the machine geometry can change in both radial and axial directions. Here, the dimensions change in the axial and radial directions by using an adjustment factor, $\mathrm{C}_{\text {adj }}$, in the form of:

$$
\begin{aligned}
& L_{s t_{-} \text {new }}=C_{a d j} \times L_{s t_{-} \text {old }} \\
& R_{\text {new }}=R_{\text {old }} / \sqrt{C_{a d j}}
\end{aligned}
$$

\subsubsection{Results}

Figure 7.13 shows the comparison of the initial and optimized back EMF waveforms. It is observed that the maximum amplitude and shape have changed. This is mainly because of the changes occurred in the pole height and inter-pole angle after optimization. Figure 7.14 shows a comparison between the cogging torques before and after optimization. Although, the maximum amplitude of the cogging torque did not change, its frequency and RMS value was decreased by $33 \%$ and $15 \%$, respectively. Figure 7.15 and figure 7.16 show the self and mutual inductances before and after optimization, respectively. The DC amplitude of the self-inductance has increased. The shape of the self-inductance is more similar to a sine wave comparing to the self-inductances in the initial design. The DC amplitude of the mutual inductances has not changed.

Figure 7.17 shows the flux linkage of the machine before and after optimization. It is seen that the maximum amplitude and shape of the flux linkage are changed.

Figure 7.18 shows the torque of the machines before and after optimization. As it 
is illustrated in this figure, the torque ripple of the optimized machine has decreased.

Figure 7.19 shows the simulated speed of the original and optimized machines. The enlarged view of speed shows that speed ripple of the optimized machine is reduced, although it was not a direct objective.

In figure 7.20 , the reference current which is inside the simulation domain and the current adjustable load (CAL) current which is measured, for initial and optimized machine are illustrated. As this figure shows, with the introduced control, an excellent compatibility between these two currents can be observed. However, a shift in measured current appears which is because of the computational delays.

Figures 7.21.a to 7.21.c show the torque versus speed profile of the initial and optimized machines calculated at three different states. Figure 7.21.a is calculated based on the assumption that the thermal and maximum drive current constraints exist in equation (7-28).

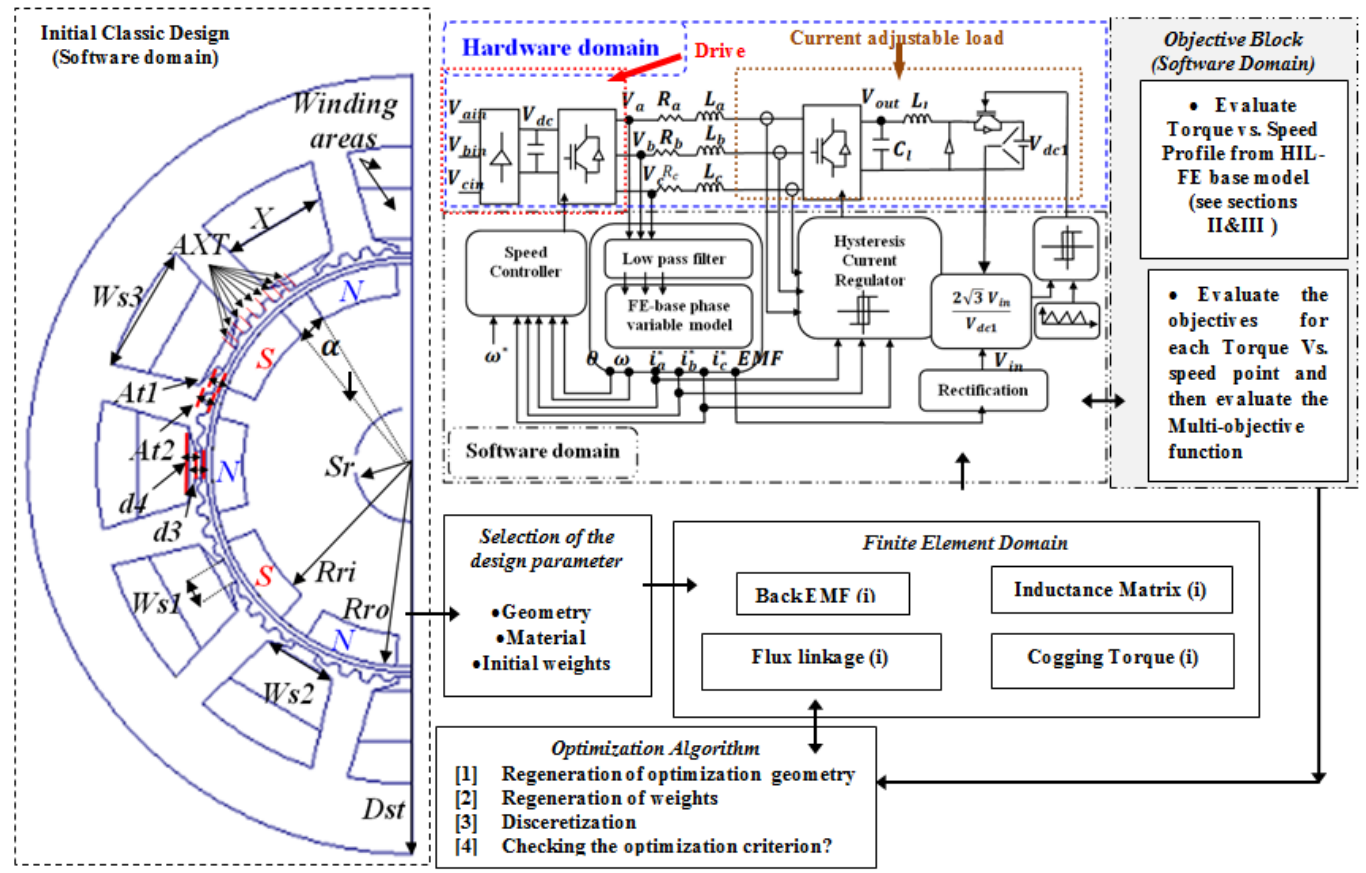

Figure 7.12 Online HIL finite element based design optimization process 
Figure 7.21.b is calculated based on the assumption that the maximum drive current constraint does not exist in equation (7-28). Figure 7.21.c is calculated based on the assumption that both of the constraints of the phase current are ignored in equation (7-28). Following the optimization, the minimum torque for the nominal speed of $600(\mathrm{rad} / \mathrm{s})$ is satisfied, the optimized machine has wider speed range while the initial design has wider torque range. From figure 7.21 it is visible that the drive system suffers from flux weakening control as well as proper speed controller bandwidth in high speed and low torque regions.

Tables 7.9, 7.10 and 7.11 show the initial and the optimal geometries of the PM motor. As can be concluded from these Tables, there is no need for auxiliary teeth number $1\left(\mathrm{AUX}_{1}\right)$. The reason is that this leads to iron saturation in the machine's teeth opening neighbor to slot opening. The auxiliary teeth number $2\left(\operatorname{AUX}_{2}\right)$ which is at the center of teeth opening is evolved to influence the back EMF as well as cogging torque. The volume of copper and magnets before and after optimization are shown in Table 12. It is observed that the copper volume, magnet volume and mass of the optimized machine are all reduced. Table 7.13 shows the objectives of optimization which are improved after optimization. Table 7.14 shows a comparison between the RMS phase current and THD. The THD of current calculated for all of the torque-speed ranges are decreased while the average RMS of phase current is increased. The average of the RMS value of the phase current is increased by $50 \%$ for all of the torque-speed points. By analysis and comparison of the normalized phase current and the back EMF waveforms of the initial and the optimized designs infigures 7.22.a and 7.22.b, it is concluded that the shape of the current waveform of the optimized machine is more close to its back EMF shape. 


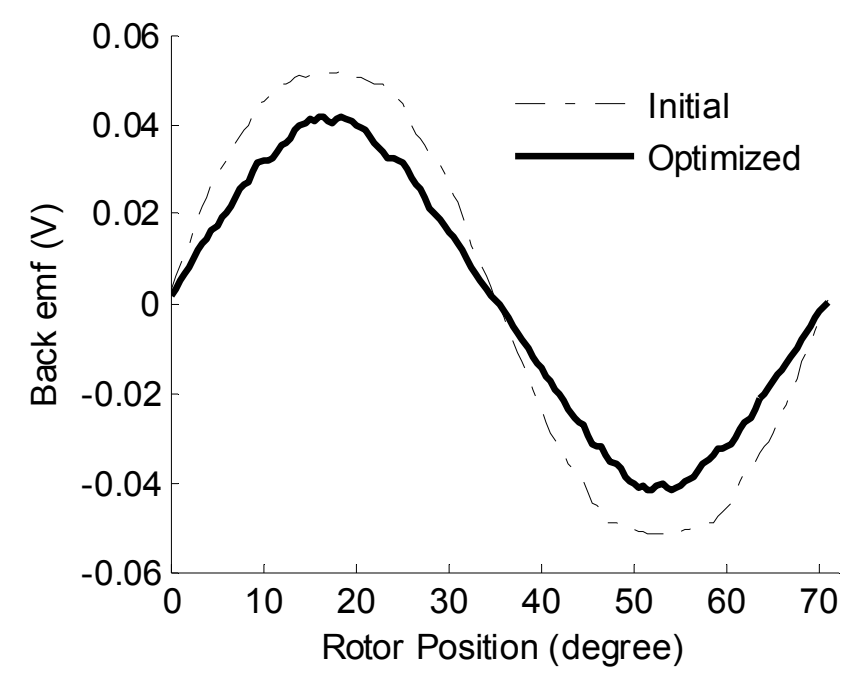

Figure 7.13 Back EMF of the phase A before and after optimization at speed of 1 $(\mathrm{rad} / \mathrm{s})$

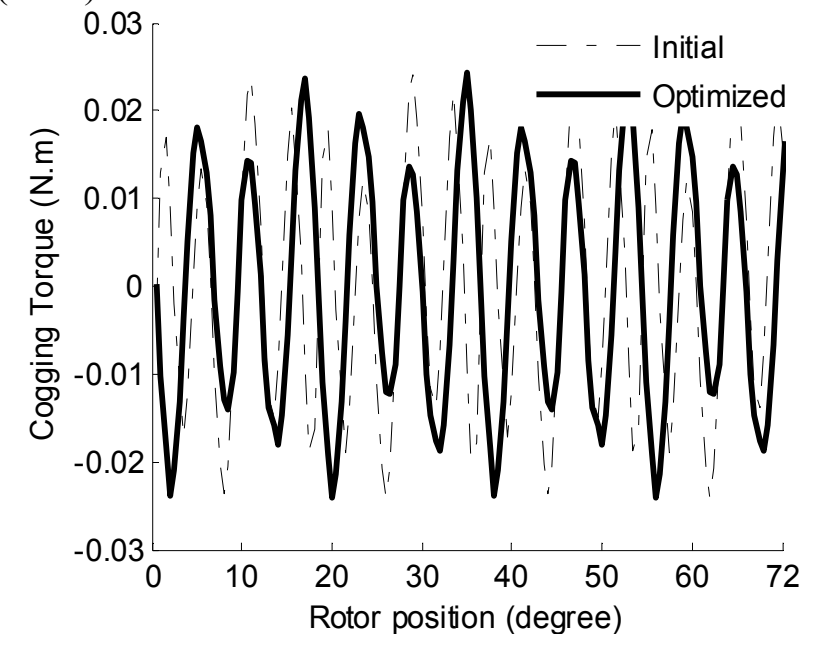

Figure 7.14 The cogging torque in initial and optimized design

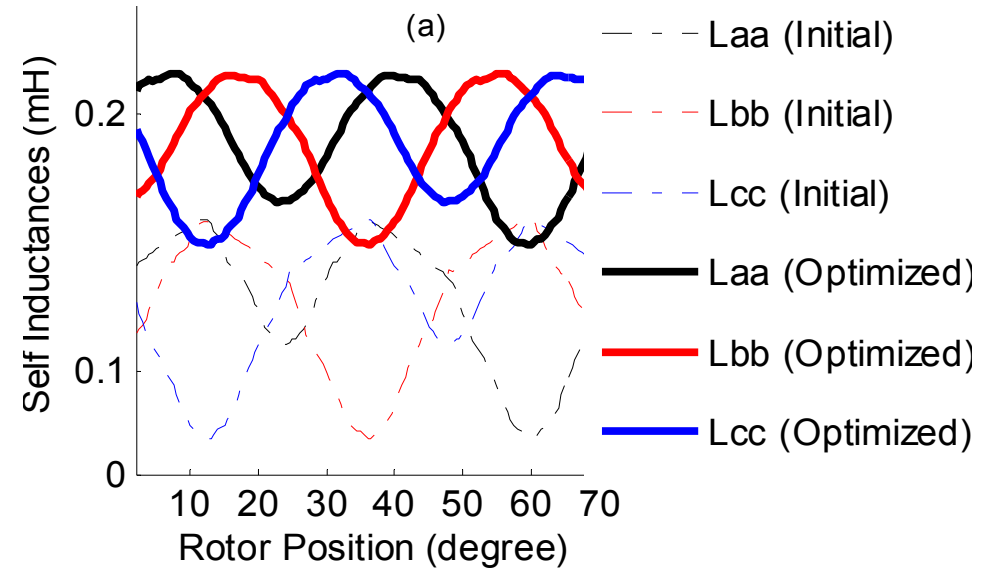

Figure 7.15 Self inductances before and after optimization 


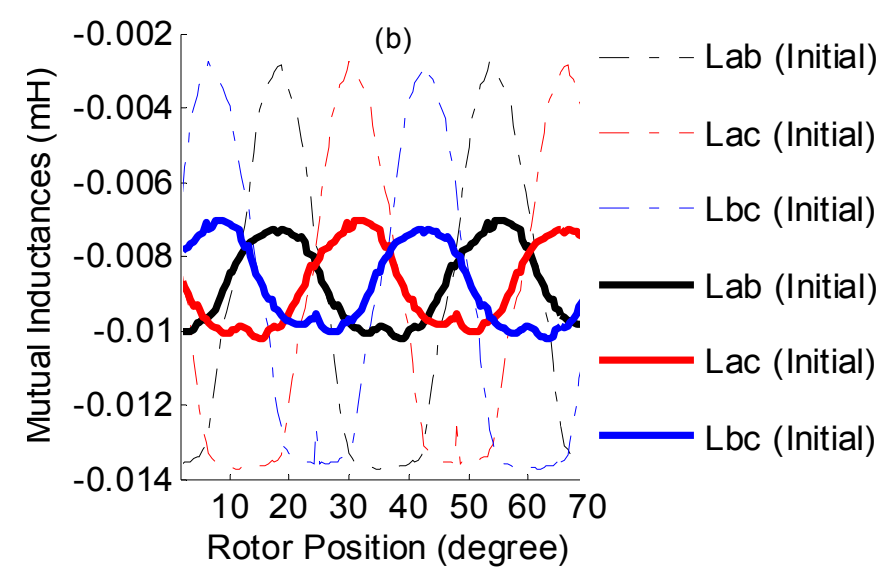

Figure 7.16 Mutual inductances before and after optimization

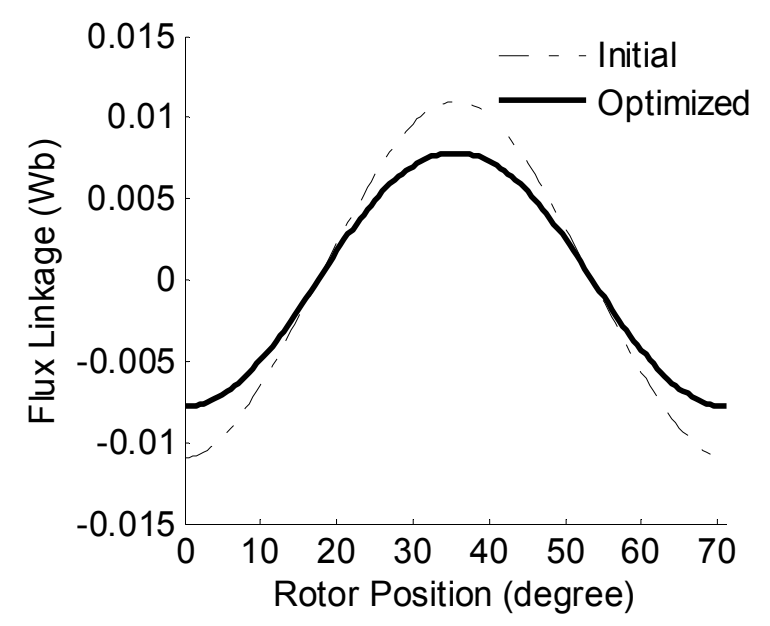

Figure 7.17 Flux linkage of the phase "a" before and after optimization

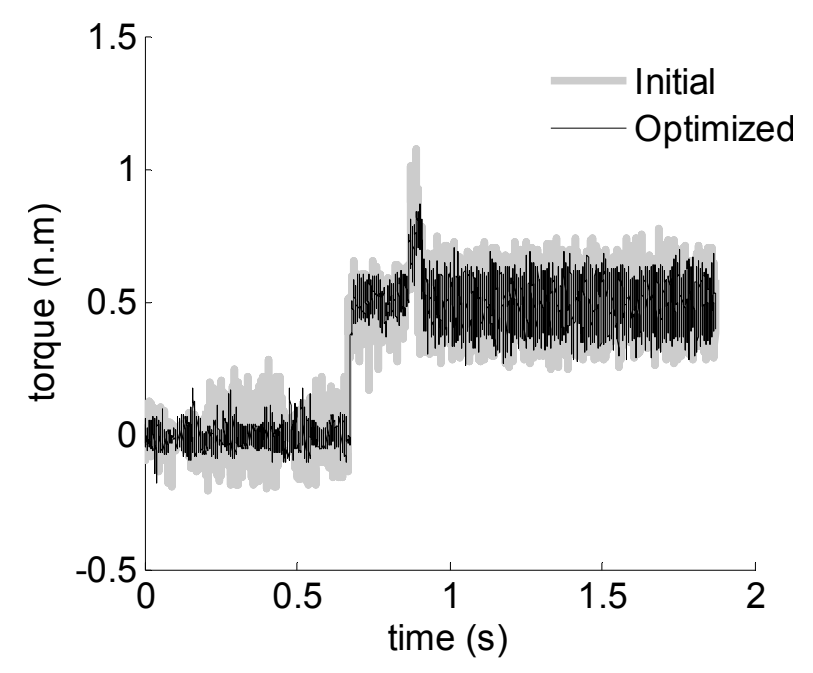

Figure 7.18 Dynamic torque measured for $0.5(\mathrm{~N}-\mathrm{m})$ and $150(\mathrm{Rad} / \mathrm{s})$ 


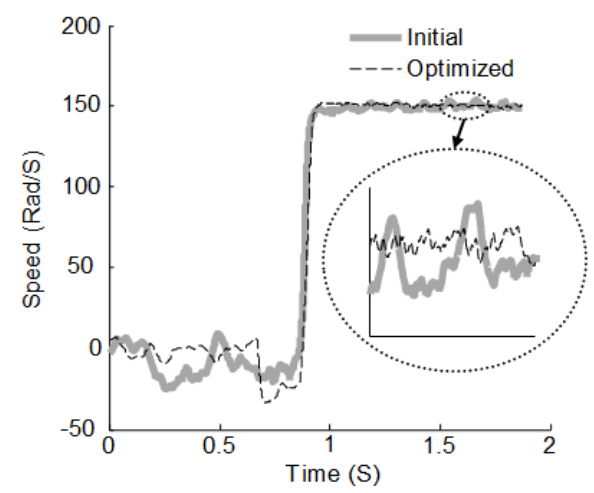

Figure 7.19 Speed dynamic measured for $0.5(\mathrm{~N}-\mathrm{m})$ and $150(\mathrm{Rad} / \mathrm{s})$

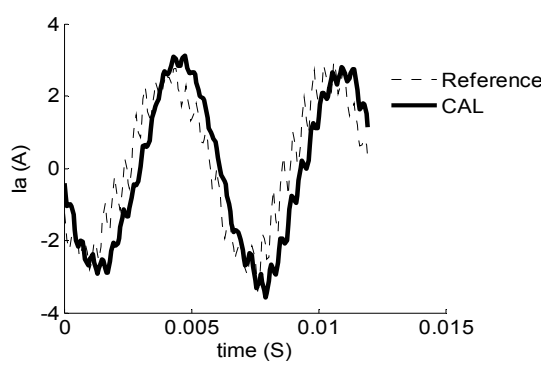

(a)

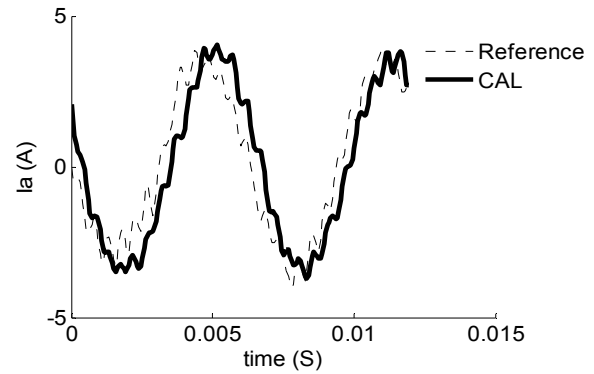

(b)

Figure 7.20 Visual comparison of the reference and current adjustable load current for optimized (b) machine at $0.5(\mathrm{~N}-\mathrm{m})$ and $300(\mathrm{rad} / \mathrm{s})$

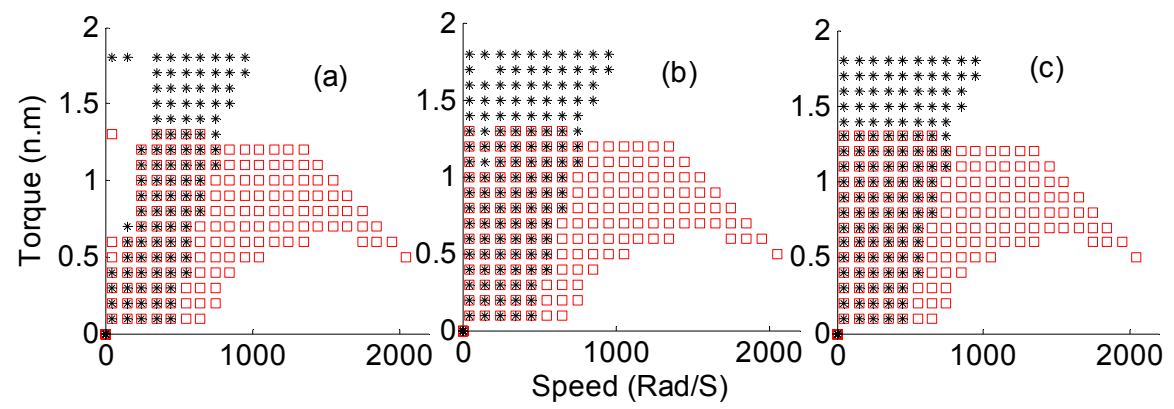

Figure 7.21 Torque Vs. speed profile, initial design (star), optimal design (square)

Table 7.8 Boundaries and constraints of optimization's input variables

\begin{tabular}{|c|c|c|c|c|}
\hline $\begin{array}{c}\text { Radial height } \\
\text { of } \mathrm{AUX}_{1} \mathbf{( m m )}\end{array}$ & $\begin{array}{c}\text { Radial height } \\
\text { of } \mathrm{AUX}_{2} \\
(\mathbf{m m})\end{array}$ & $\begin{array}{c}\text { Inter-pole } \\
\text { angle } \\
(\mathbf{d e g r e e})\end{array}$ & $\begin{array}{c}\text { Pole height }\left(\mathrm{h}_{\mathrm{m}}\right) \\
\mathbf{( m m )}\end{array}$ & $\mathrm{B}_{\text {yoke }}[\mathrm{T}]$ \\
\hline$[0,0.5]$ & {$[0,2.4]$} & {$[0,10]$} & {$[2.5,4.5]$} & {$[0,1.3]$} \\
\hline $\mathrm{C}_{\mathrm{f}}$ & $\mathrm{D}_{\mathrm{st}}(\mathrm{mm})$ & $\mathrm{I}(\mathrm{A})$ & $\mathrm{L}_{\mathrm{st}}(\mathrm{mm})$ & $\mathrm{J}\left(\mathrm{A} / \mathrm{mm}^{2}\right)$ \\
\hline$[0.9,1.1]$ & {$[35-42]$} & 15 & {$[65-85]$} & 2 \\
\hline
\end{tabular}


Table 7.9 Stator dimension before and after optimization $(\mathrm{mm})$

\begin{tabular}{|c|c|c|c|c|c|c|c|}
\hline Stator dimension & $D_{s t}$ & $D$ & $d_{3}$ & $d_{4}$ & $W_{s 1}$ & $W_{s 2}$ & $W_{s 3}$ \\
\hline Initial(mm) & 37.5 & 8.4 & 0.65 & 0.65 & 2.5 & 7.3 & 12.4 \\
\hline Optimized(mm) & 39.3 & 8.4 & 0.65 & 0.65 & 2.5 & 7.3 & 12.4 \\
\hline
\end{tabular}

Table 7.10 Stator dimension before and after optimization $(\mathrm{mm})$

\begin{tabular}{|c|c|c|c|c|c|c|}
\hline $\begin{array}{c}\text { Stator } \\
\text { dimension }\end{array}$ & Stack length $\left(L_{s t}\right)$ & $A t_{1}$ & $A t_{2}$ & $A U X$ & turns / phase & Ns / phase \\
\hline Initial(mm) & 35 & 0 & 0 & 0 & 48 & 4 \\
\hline Optimized(mm) & 30.1 & 0 & 0.4 & 1 & 48 & 4 \\
\hline
\end{tabular}

Table 7.11 Rotor dimension 12 slot/10 poles, SPM machine (mm)

\begin{tabular}{|c|c|c|c|c|c|c|}
\hline Rotor Dimension & $R_{r i}$ & $S_{R}$ & $g$ & $R_{r o}$ & $L_{m}$ & $\alpha$ (degree) \\
\hline Initial(mm) & 16.4 & 5.5 & 1 & 19.5 & 3 & 0 \\
\hline Optimized(mm) & 17.6 & 5.7 & 1 & 20.4 & 2.8 & 6.3 \\
\hline
\end{tabular}

Table 7.12 Volumes and mass before and after optimization

\begin{tabular}{|c|c|c|c|c|}
\hline Machine & $\begin{array}{c}\text { Vol. of copper/slot } \\
\left(\mathrm{mm}^{3}\right)\end{array}$ & $\begin{array}{c}\text { Vol. of Magnet } \\
\text { pole }\left(\mathrm{mm}^{3}\right)\end{array}$ & $\begin{array}{c}\text { Mass } \\
(\mathrm{gr})\end{array}$ & $\begin{array}{c}\text { Moment of inertia } \\
\left(\mathrm{Kg}^{2} \mathrm{~m}^{2}\right)\end{array}$ \\
\hline Original & 2667 & 1204.6 & 302 & $6.2715 \mathrm{e}-5$ \\
\hline Optimized & 2293.4 & 1061.2 & 282 & $6.3962 \mathrm{e}-5$ \\
\hline Saved (\%) & 14.02 & 11.88 & 6.62 & ------- \\
\hline
\end{tabular}

Table 7.13 Average performance before and after optimization

\begin{tabular}{|c|c|c|c|c|c|c|}
\hline Performances & $\begin{array}{c}\text { Mean THD } \\
\%\end{array}$ & $\begin{array}{c}T_{\text {ripple }} \\
(\mathrm{N} . \mathrm{m})\end{array}$ & $\begin{array}{c}I_{R M S} \\
(\mathrm{~A})\end{array}$ & $K_{m}$ & $\begin{array}{c}R_{a} \\
(\Omega)\end{array}$ & $\begin{array}{c}\mathrm{J} \\
\left(\mathrm{A} / \mathrm{mm}^{2}\right)\end{array}$ \\
\hline Initial & 7.484 & 0.196 & 2.03 & 0.343 & 0.8803 & 1.5485 \\
\hline Optimum & 5.17 & 0.123 & 2.95 & 0.168 & 0.757 & 2.23 \\
\hline
\end{tabular}

In the initial design, the back EMF was the envelope of the current waveform while in the optimized machine show that this order is changed. In fact this helps for wider torque-speed profile as illustrated in figure 7.21. Moreover, by visual 
comparison of the current waveform and the back EMF waveform at the vicinity of the peak of the back EMF, more matching occur after optimization. In fact, the maximum of energy conversions are occurred at the vicinities of the peak of the back EMF.

Table 7.1 Comparison of THD and RMS of phase current for different levels of torque and speed

\begin{tabular}{|c|c|c|c|c|c|c|c|}
\hline (speed, torque) & $150,0.5$ & $200,0.5$ & $250,0.5$ & $300,0.5$ & $350,0.5$ & $400,0.5$ \\
\hline RMS(A) & 1.03 & 1.35 & 1.71 & 2.07 & 2.6886 & 3.31 \\
\hline THD\% & 13 & 9.84 & 6 & 4 & 6.56 & 5 \\
\hline & & \multicolumn{5}{|c|}{ Optimized } \\
\hline RMS & 1.5 & 2.07 & 2.5 & 3.18 & 3.85 & 4.5 \\
\hline THD\% & 9.05 & 5.75 & 4.45 & 2.72 & 5.53 & 3.56 \\
\hline
\end{tabular}

The drive topology used in the experiments was a conventional laboratory-based three phase 6-switch drive supplied with the fixed DC voltage as shown earlier in figure 7.11 and 7.12. However, any three phase voltage-source inverter with controllable DC voltage can be used in its place. From this point of view, all of the limitations for adjusting the current fore the adjustable load should be set for the maximum possible DC bus voltage.

In the optimization process, the thermal aspect is controlled by limiting the current density. However, for a more realistic optimization, the equivalent thermal model of the machine can be added to the FE-based phase variable model.

The 3-D view of the initial design and the optimized design are illustrated infigure 7.23. Although there is not a major difference between the initial and the final designs in terms of the geometrical shape and the size, the optimization process could fit the performance of the PMSM-drive system to the desired goals. The field picture of the initial and the final designs are depicted infigures 7.24 and 7.25. It is seen that the 
concentration of flux in the rotor back iron is reduced in the optimized machine. In addition, the flux leakage in inter-pole area is reduced.

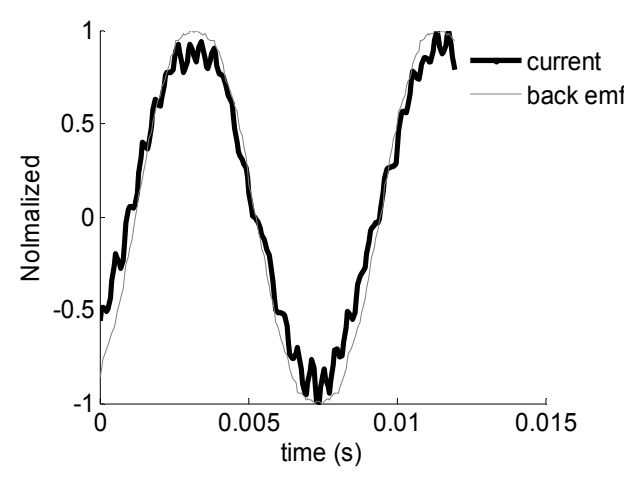

(a)

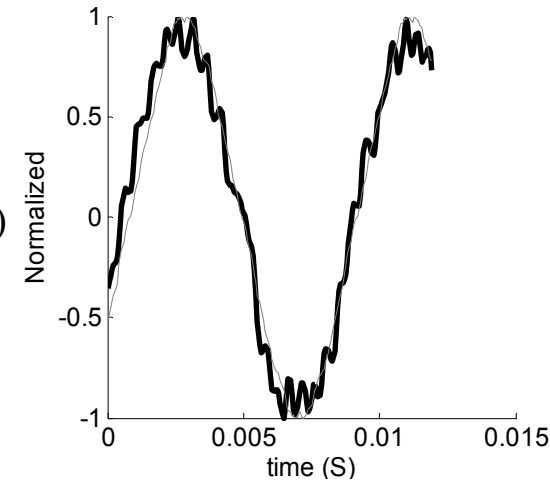

(b)

Figure 7.22 The normalized current and back EMF, initial (a) and optimized (b)

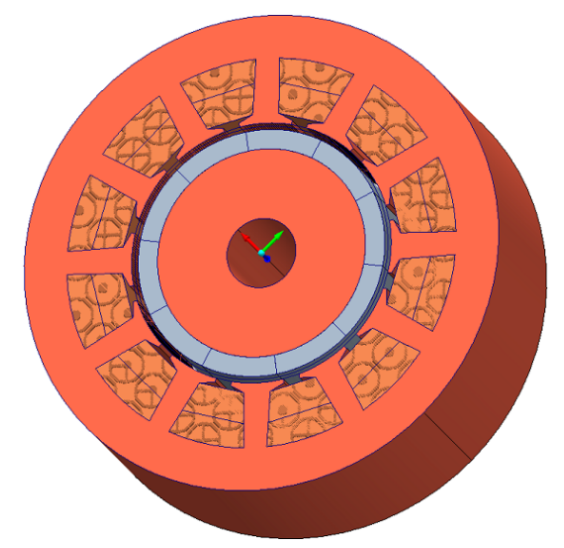

(a)

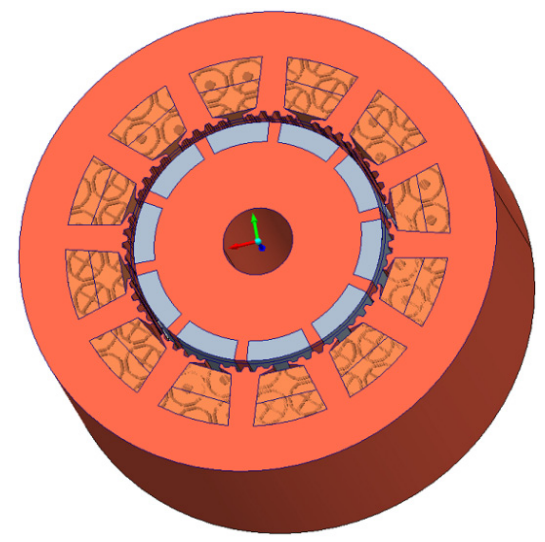

(b)

Figure 7.23 3-D view of the initial (a) and the optimized machine(b)

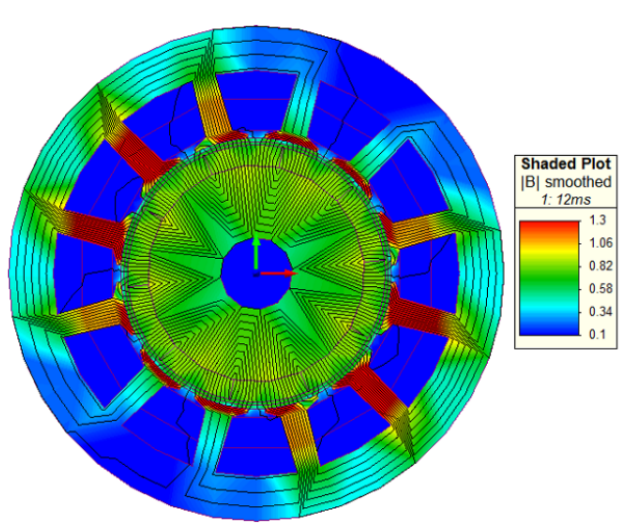

(a)

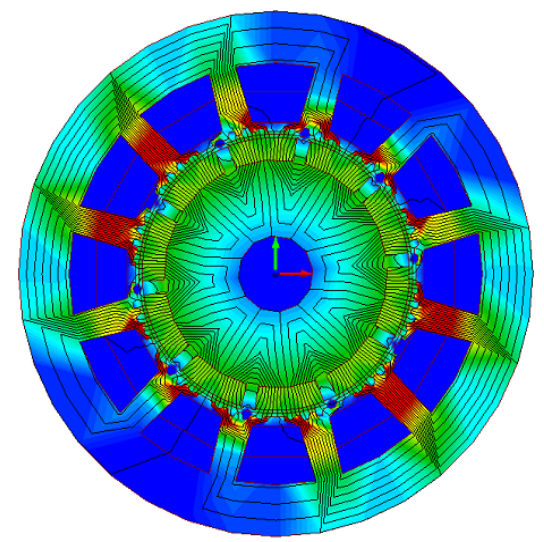

(b)

Figure 7.24 Field picture and absolute flux density of the initial and the optimized machine 


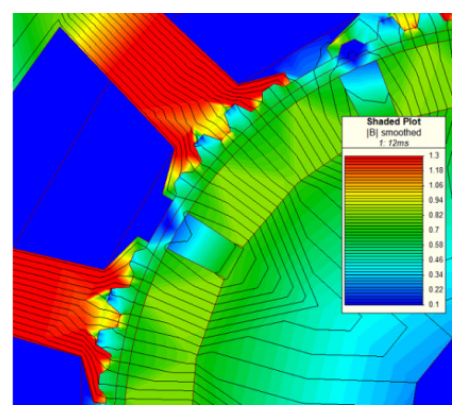

(a)

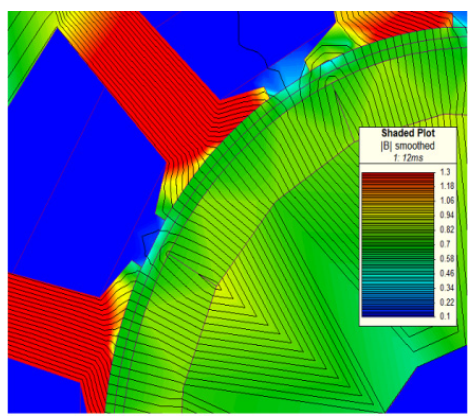

(b)

Figure 7.25 Field picture and absolute flux density of the initial and the optimized machine in the inter-pole angle areas

In the HIL-optimization process, some of the geometrical design parameters of the machine were introduced as the optimization variables and the current drive circuit was seen as a black box. This is helpful where the machine manufacture needs to make the design of their machines to be most compatible to a commercial drive existing in the market. Moreover, the introduced technique was developed for PMSMdrive systems. Similar approaches can be performed for other electromagnetic systems with similar prospective where the compatibility of existing or being developed parts needs to be experimentally verified before actual prototyping.

During optimization two hundred possible solutions is calculated. The optimization algorithm converged after 130000 second. The used CPU and memory are $1.17 \mathrm{~GB}$ and $3 \mathrm{GHz}$, respectively. The complexity of the geometry and number of the freedom in FE domain are the main factors that influence the time elapsed in the optimization process.

\subsection{Summary}

In this chapter, the performance enhancement of PMSM machine using a hybrid multi-objective optimization was developed where the objectives were calculated from the physics-based model of the machine. A dynamic physics-based phase variable model of the machine, as a fast and accurate model, was used to indirectly 
link the machine design parameters to its drive circuit during optimization process, in this way, the dynamic effect of PWM IGBT sinusoidal current drive on machine performance during design process was taken into account. Following optimization, the area of copper, the area of magnet, the torque ripple, the speed ripple, the total harmonic distortion of the phase currents, the total mass were reduced while the motor constant was increased. In addition, the development of an online HIL based finite element intelligent optimization process was presented. The application of this optimization process was implemented in a laboratory-based experimental setup. We have also shown how a current adjustable load was used as an equivalent hardware representation of a motor drive example and can it be employed in an online population-based optimization process of the machine-drive system. It was also shown that the dynamic physics-based phase variable model of the machine, as a fast and accurate model can be used to indirectly link the machine design parameters to its drive circuit during a HIL based optimization process. In this optimization process, the dynamics effects of the drive on the machine performance during the design stage were taken into account. The performance measures as well as the physical properties of the example machine drive before and after optimization were compared. It was observed that after the optimization, the area of copper, the area of magnet, the torque ripple, the speed ripple, the total harmonic distortion of the phase currents and the total mass were all improved in the final design. In addition, it was observed that the separate design of PM magnet machine without mentioning the dynamic and the constraints of drive may not satisfy the desirable performances. 


\section{Chapter 8 Discussion of Results, Conclusions and Future Work}

\subsection{Conclusions}

In this dissertation, the application of physics-based model of electric machines for the purpose of co-design, control and fault diagnosis were investigated. In particular, permanent magnet machine-drive systems were comprehensively investigated for maximum fault tolerance and optimal operation.

The classic design of a permanent magnet synchronous machine without saliency was introduced. It was shown that the slot pitch, pole pitch, magnet demagnetization and the dimension of the machine in addition to the number of the turns/coil were the factors that affected the flux linkage. As a result, the back EMF of the machine was also affected. The back EMF directly affects the power of the machine. Finding the best back EMF shape and amplitude for a nominal speed and torque is a multiobjective optimization process which considers combining the cogging torque, radial force and thermal limitations. Although, the optimization process can be launched from the introduced classic design procedure for desired objectives such as minimum cogging torque, minimum radial force and proper back EMF waveforms. The difficulties in the accurate calculation of the back EMF harmonics, cogging torque amplitude and radial force amplitude, encourage us to expand the design problem into a numerical domain. In addition, the mutual integration of the drive and a machine being developed is one of the topics that cannot be easily performed from analytical expressions. Therefore, the classic design seems to be just a reasonable state for initialization of the design. In other words, the classic design enables one to quickly obtain a normal estimation of the final design. In such an initial design, the general geometry of the machine, the initial dimension of the machine and the material for a 
nominal operating point, are calculated based on the knowledge presented earlier in chapter 2 of this dissertation. The final design was produced from the advanced optimization process, which covers higher dimension objectives, was presented in chapter 7 .

The fundamentals of finite elements, the creation of embedded thermal and electrical models, required for the development of the physics-based optimization tools, the open loop observers and the estimation process were presented in chapter 3 . Several novel contributions including the voltage based inductance calculation method, the embedded thermo-electromagnetic model of the machine, the magnetic monitoring of the machine and inclusion of the effect of skewing in $2 \mathrm{D}$ finite element analysis were introduced.

The current technologies in the PMSM drive systems were reviewed in chapter 4. In addition, the developed models of chapter 3 were employed for real-time demagnetization control of three interior permanent magnet machine designs. The permanent magnet (PM) demagnetization control for the optimal and reliable flux weakening control in PMSM was presented. A physics-based modeling approach was developed to estimate the magnetic operating point of the PM poles during the operation of PMSM. The model was enabled to dynamically estimate the average and the partial PM demagnetization due to reverse armature field as well as the PM over temperature. The demagnetization assessment and control were verified on a V-type PMSM using the modified FE-based coupled thermal-field-circuit phase variable model and the time stepping FE analysis. Three PMSM designs were compared concerning the demagnetization issue. It was observed that increasing the flux concentration factor in the design of PMSM by enlarging the magnet contact area would increase the risk of demagnetization. It was numerically proven that the real- 
time demagnetization control theory can lead to a large savings in permanent magnet size as well as the iron core without compromising in the reliability of the electrical machine.

Using the developed model in chapter 3, a sensorless rotor position estimation and control were developed to increase the applicability of the EMF based observers for medium-low, low and very low speeds. A novel thermoelectric physics-based modeling approach was developed to evaluate more realistic information about the instantaneous behavior of the machine during operation. For removing the high frequency noise during angle calculations without missing the observer band-width, an adaptive angle compensator was designed. The simulation and experimental results under different speed, loading and voltage variation show acceptable accuracy. The proposed physics-based sensorless drive provides a rotor-position estimate, which is invulnerable to system noise. It can also estimate the precise speed, because it can follow the machine model variations. The temperature was estimated using an embedded transient thermal model. The introduced sensorless control eliminates the need for simultaneous observation of the stator resistance, stator inductance and the rotor flux magnitude.

Using the developed model in chapter 3, a physics-based development of an open loop EMF estimator was presented in chapter 6 . The fault detection based EMF analysis due to inter-turn fault was studied and discussed. It was observed that the method performed in a stable manner for the detection against the blurring effects of the harmonic loads and dynamic eccentricity for wide speed ranges. It was also observed that the EMF can determine the intensity and the location of a faulty phase in a fraction of second. This method allows prompt and online stator-fault detection from the real-time estimated EMF without additional computationally heavy signal 
processing methods. The developed method show acceptable results for low speeds where the inter-turn fault reflection on the performance of the machine, phase current and the voltage at the terminals are not easily sensible. The results show acceptable performance in fault detection for wide speed ranges even in the presence of harmonic loads and dynamic eccentricities.

Two noble design optimization schemes for the co-design of radial flux electrical permanent magnet machine and drive system were developed in chapter 7. It was shown that the software-based optimization scheme has a unique ability in invoking the finite element based software inside MATLAB/SIMULINK analysis, where large computational as well as hardware interfaces tools are available. This ability enabled one to extend the optimization scheme into hardware in the loop finite element based design optimization scheme, where there is a need to design an electrical machine for an available drive system. In the hardware in the loop finite element based design optimization scheme, the electrical machine was practically modeled by a current adjustable load. The current adjustable load was practically built and it was connected to an available drive. A typical permanent magnet machine was designed using the procedure introduced in chapter 2 . This procedure was successfully optimized using the hardware in the loop finite element based design optimization scheme. It was shown that the hardware in the loop finite element based optimization scheme has a unique ability to particularly optimize a machine for an existing drive in the market with respect to the desired dynamic responses and drive constraints. The developed physics-based model of the machine, as a fast and accurate model, was used to indirectly link the machine design parameters to its drive circuit during optimization process. In this way, the dynamic effect of PWM IGBT sinusoidal current drive on machine performance during design process was taken into account. Following the 
optimization, the area of copper, the area of magnet, the torque ripple, the speed ripple, the total harmonic distortion of the phase currents and the total mass were reduced while the motor constant was increased. In the hardware in the loop optimization process, the dynamics effects of the drive on the machine performance during the design stage were taken into account. The performance measures as well as the physical properties of the example machine drive before and after optimization were compared. It was observed that after the optimization, the area of copper, the area of magnet, the torque ripple, the speed ripple, the total harmonic distortion of the phase currents and the total mass were improved in the final design. In addition, it was observed that the separate design of PM magnet machine without mentioning the dynamics and the constraints of the drive may not satisfy the desirable performances.

\subsection{Future work}

The developed physics-based model of PMSM was enabled to thermally, electrically and magnetically monitor the machine in real-time under different operational contingencies with high accuracy. This ability was used to develop very robust and accurate open loop observers. Similar ideas can be developed for modelbased control development of electrostatic and similar electromagnetic devices where in case the driving of such systems affects the life time and the performance of the device.

The developed ideas in the design of permanent magnet machine-drive systems were applied in three phase radial flux permanent magnet machine. Similar procedure can be applied to other machines with similar prospective. In this sense, the design and optimization of the induction machine-drive systems and switched reluctance machine drive systems will be the case for future research.

Because of the practical implementation and studying all the scenarios in real-time 
demagnetization control are estimated as a very costly process, it was merely applied in a software domain. However, the author seeks to practically test it for PMSM where the price of permanent magnet materials plays a vital role in the selection of such that device in the traction drive systems. 


\section{LIST OF REFERENCES}

[1] D. M. Ionel, "Design considerations for PM synchronous motors for flux weakening applications," in Eighth International Conference on Electrical Machines and Drives, 1997, vol. 1997, pp. 371-375.

[2] N. C. Harris and T. M. Jahns, "Design of an integrated motor/controller drive for an automotive water pump application," in Conference Record of the 2002 IEEE Industry Applications Conference. 37th IAS Annual Meeting (Cat. No.02CH37344), vol. 3, pp. 2028-2035.

[3] T. Sebastiangordon and G. R. Slemon, "Operating Limits of Inverter-Driven Permanent Magnet Motor Drives," IEEE Transactions on Industry Applications, vol. IA-23, no. 2, pp. 327-333, Mar. 1987.

[4] A. M. El-Refaie and T. M. Jahns, "Comparison of synchronous PM machine types for wide constant-power speed operation: converter performance," IET Electric Power Applications, vol. 1, no. 2, p. 217, 2007.

[5] K. Lee, K. Kim, S. Kim, J.-S. Ahn, S. Lim, and J. Lee, “Optimal magnet shape to improve torque characteristics of interior permanent magnet synchronous motor," Journal of Applied Physics, vol. 97, no. 10, p. 10Q505, 2005.

[6] Z. Lei, W. Xuhui, Z. Feng, and Y. Yi, "Voltage and power limitations of generation systems with uncontrolled PMSMs and DC/DC converters," in 2011 IEEE Vehicle Power and Propulsion Conference, 2011, pp. 1-5.

[7] L. . Parsa and H. A. Toliyat, "Fault-Tolerant Interior-Permanent-Magnet Machines for Hybrid Electric Vehicle Applications," IEEE Transactions on Vehicular Technology, vol. 56, no. 4, pp. 1546-1552, Jul. 2007.

[8] A. M. El-Refaie, "Fault-tolerant permanent magnet machines: a review," IET Electric Power Applications, vol. 5, no. 1, p. 59, 2011.

[9] N. Higuchi, K. Kaiho, I. Ishii, H. Nomura, H. Tateishi, S. Fuchino, K. Arai, S. Sekine, N. Natori, K. Kajikawa, N. Tamada, T. Onishi, T. Aiba, and T. Iimura, "A study on stability of rotating magnets," IEEE Transactions on Magnetics, vol. 32, no. 4, pp. 2385-2388, Jul. 1996.

[10] D. O. Koval and H. L. Floyd, "Human element factors affecting reliability and safety," IEEE Transactions on Industry Applications, vol. 34, no. 2, pp. 406414, 1998.

[11] P. H. Mellor, M. A. Al-Taee, and K. J. Binns, "Open loop stability characteristics of synchronous drive incorporating high field permanent magnet motor," vol. 138, 1991. 
[12] E. M. Rashad, "Stability Limits of Saturated Interior Permanent Magnet Motors," in 2005 International Conference on Power Electronics and Drives Systems, vol. 1, pp. 584-589.

[13] "General Principles of Winding Failure, Repair, and Rewinding ," pp. 129136.

[14] “Rotor Winding Failure Mechanisms and Repair ," pp. 181-209.

[15] B. Schwarz, "Geometrical approach to the economical design of rotating electrical machines," Proceedings of the Institution of Electrical Engineers, vol. 113, no. 3, p. 493, 1966.

[16] B. C. Mecrow, A. G. Jack, J. A. Haylock, and J. Coles, "Fault-tolerant permanent magnet machine drives," IEE Proceedings - Electric Power Applications, vol. 143, no. 6, p. 437, 1996.

[17] C. E. Webb, "The stability of permanent magnets," Proceedings of the IEE Part C: Monographs, vol. 108, no. 14, p. 317, 1961.

[18] A. J. Tyrrell, "The performance and stability of permanent magnets," vol. 10, 1950.

[19] T. Tanaka, "Aging of polymeric and composite insulating materials. Aspects of interfacial performance in aging," IEEE Transactions on Dielectrics and Electrical Insulation, vol. 9, no. 5, pp. 704-716, Oct. 2002.

[20] D. J. O'Connor, 'Impact of microstructure on stability of permanent magnet biased magnetoresistive heads," IEEE Transactions on Magnetics, vol. 32, no. 1, pp. 54-60, 1996.

[21] M. McCaig, "Stability of chromium-iron-cobalt permanent magnet alloys," IEEE Transactions on Magnetics, vol. 11, no. 5, pp. 1443-1445, Sep. 1975.

[22] S. Ruoho, J. Kolehmainen, J. Ikaheimo, and A. Arkkio, "Interdependence of Demagnetization, Loading, and Temperature Rise in a Permanent-Magnet Synchronous Motor," IEEE Transactions on Magnetics, vol. 46, no. 3, pp. 949953, Mar. 2010.

[23] G. D. Demetriades, E. Andersson, and H. Olsson, "A Real-Time Thermal Model of a Permanent Magnet Synchronous Motor Based on geometrical measures," in 2008 IEEE Power Electronics Specialists Conference, 2008, pp. 3061-3067.

[24] S. Ruoho, J. Kolehmainen, J. Ikaheimo, and A. Arkkio, "Interdependence of Demagnetization, Loading, and Temperature Rise in a Permanent-Magnet Synchronous Motor," IEEE Transactions on Magnetics, vol. 46, no. 3, pp. 949953, Mar. 2010. 
[25] A. Yazidi, H. Henao, G.-A. Capolino, F. Betin, and L. Capocchi, "Inter-turn short circuit fault detection of wound rotor induction machines using Bispectral analysis," in 2010 IEEE Energy Conversion Congress and Exposition, 2010, pp. $1760-1765$.

[26] T. M. Wolbank, K. A. Loparo, and R. Wohrnschimmel, "Inverter statistics for online detection of stator asymmetries in inverter-fed induction motors," IEEE Transactions on Industry Applications, vol. 39, no. 4, pp. 1102-1108, Jul. 2003.

[27] B. Vaseghi, N. Takorabet, and F. Meibody-Tabar, "Fault Analysis and Parameter Identification of Permanent-Magnet Motors by the Finite-Element Method," IEEE Transactions on Magnetics, vol. 45, no. 9, pp. 3290-3295, Sep. 2009.

[28] F. C. Trutt, J. Sottile, and J. L. Kohler, "Online condition monitoring of induction motors," IEEE Transactions on Industry Applications, vol. 38, no. 6, pp. 1627-1632, Nov. 2002.

[29] Greg C. Stone, Edward A. Boulter, Ian Culbert, H. Dhirani, "General Principles of Testing and Monitoring," Wiley Online liberary, pp. 227-233.

[30] J. A. Haylock, B. C. Mecrow, A. G. Jack, and D. J. Atkinson, "Operation of fault tolerant machines with winding failures," IEEE Transactions on Energy Conversion, vol. 14, no. 4, pp. 1490-1495, 1999.

[31] J. D. Ede, K. Atallah, and D. Howe, "Effect of optimal torque control on rotor loss of fault-tolerant permanent-magnet brushless machines," IEEE Transactions on Magnetics, vol. 38, no. 5, pp. 3291-3293, Sep. 2002.

[32] P. Arumugam, T. Hamiti, and C. Gerada, "Modeling of Different Winding Configurations for Fault-Tolerant Permanent Magnet Machines to Restrain Interturn Short-Circuit Current," IEEE Transactions on Energy Conversion, vol. 27, no. 2, pp. 351-361, Jun. 2012.

[33] T. Raminosoa, C. Gerada, and M. Galea, "Design Considerations for a FaultTolerant Flux-Switching Permanent-Magnet Machine," IEEE Transactions on Industrial Electronics, vol. 58, no. 7, pp. 2818-2825, Jul. 2011.

[34] J. Wang, K. Atallah, and D. Howe, "Optimal torque control of fault-tolerant permanent magnet brushless machines," in Digest of INTERMAG 2003. International Magnetics Conference (Cat. No.03CH37401), p. FBI-F03.

[35] W. Zhao, M. Cheng, W. Hua, H. Jia, and R. Cao, "Back-EMF Harmonic Analysis and Fault-Tolerant Control of Flux-Switching Permanent-Magnet Machine With Redundancy," IEEE Transactions on Industrial Electronics, vol. 58, no. 5, pp. 1926-1935, May 2011. 
[36] G. J. Atkinson, B. C. Mecrow, A. G. Jack, D. J. Atkinson, P. Sangha, and M. Benarous, "The Analysis of Losses in High-Power Fault-Tolerant Machines for Aerospace Applications," IEEE Transactions on Industry Applications, vol. 42, no. 5, pp. 1162-1170, Sep. 2006.

[37] T. G. Habetler, "Current-based condition monitoring of electrical machines in safety critical applications," in 2008 13th International Power Electronics and Motion Control Conference, 2008, pp. 21-26.

[38] C. Bianchini, E. Fornasiero, T. N. Matzen, N. Bianchi, and A. Bellini, "Fault detection of a five-phase Permanent-Magnet machine," in 2008 34th Annual Conference of IEEE Industrial Electronics, 2008, pp. 1200-1205.

[39] B. Akin, S. Choi, U. Orguner, and H. A. Toliyat, "A Simple Real-Time Fault Signature Monitoring Tool for Motor-Drive-Embedded Fault Diagnosis Systems," IEEE Transactions on Industrial Electronics, vol. 58, no. 5, pp. 1990-2001, May 2011.

[40] R. M. Tallam, T. G. Habetler, and R. G. Harley, "Stator winding turn-fault detection for closed-loop induction motor drives," IEEE Transactions on Industry Applications, vol. 39, no. 3, pp. 720-724, May 2003.

[41] R. M. Tallam and T. G. Habetler, "A robust, on-line turn-fault detection technique for induction machines based on monitoring the sequence component impedance matrix," IEEE Transactions on Power Electronics, vol. 18, no. 3, pp. 865-872, May 2003.

[42] J. Sottile and J. L. Kohler, "An on-line method to detect incipient failure of turn insulation in random-wound motors," IEEE Transactions on Energy Conversion, vol. 8, no. 4, pp. 762-768, 1993.

[43] J. Pons-Llinares, J. A. Antonino-Daviu, M. Riera-Guasp, M. Pineda-Sanchez, and V. Climente-Alarcon, "Induction Motor Diagnosis Based on a Transient Current Analytic Wavelet Transform via Frequency B-Splines," IEEE Transactions on Industrial Electronics, vol. 58, no. 5, pp. 1530-1544, May 2011.

[44] P. Neti and S. Nandi, "Stator Inter-turn Fault Detection of Synchronous Machines Using Field Current Signature Analysis," in Conference Record of the 2006 IEEE Industry Applications Conference Forty-First IAS Annual Meeting, 2006, vol. 5, pp. 2360-2367.

[45] O. A. Mohammed, S. Liu, and Z. Liu, "Physical modeling of PM synchronous motors for integrated coupling with Machine drives," IEEE Transactions on Magnetics, vol. 41, no. 5, pp. 1628-1631, May 2005.

[46] Y. Lee and T. G. Habetler, "An On-Line Stator Turn Fault Detection Method for Interior PM Synchronous Motor Drives," in APEC 07 - Twenty-Second 
Annual IEEE Applied Power Electronics Conference and Exposition, 2007, pp. $825-831$.

[47] N. Leboeuf, T. Boileau, B. Nahid-Mobarakeh, G. Clerc, and F. Meibody-Tabar, "Real-Time Detection of Interturn Faults in PM Drives Using Back-EMF Estimation and Residual Analysis," IEEE Transactions on Industry Applications, vol. 47, no. 6, pp. 2402-2412, Nov. 2011.

[48] G. B. Kliman, W. J. Premerlani, R. A. Koegl, and D. Hoeweler, "A new approach to on-line turn fault detection in AC motors," in IAS '96. Conference Record of the 1996 IEEE Industry Applications Conference Thirty-First IAS Annual Meeting, vol. 1, pp. 687-693.

[49] A. Khoobroo and B. Fahimi, "A new method of fault detection and treatment in five phase permanent magnet synchronous machine using field reconstruction method," in 2009 IEEE International Electric Machines and Drives Conference, 2009, pp. 682-688.

[50] C. S. Kallesoe, R. Izadi-Zamanabadi, P. Vadstrup, and H. Rasmussen, "Observer-Based Estimation of Stator-Winding Faults in Delta-Connected Induction Motors: A Linear Matrix Inequality Approach," IEEE Transactions on Industry Applications, vol. 43, no. 4, pp. 1022-1031, 2007.

[51] C. Gerada, K. Bradley, and M. Sumner, "Winding turn-to-turn faults in permanent magnet synchronous machine drives," in Fourtieth IAS Annual Meeting. Conference Record of the 2005 Industry Applications Conference, 2005., vol. 2, pp. 1029-1036.

[52] A. Garcia-Perez, R. de J. Romero-Troncoso, E. Cabal-Yepez, and R. A. Osornio-Rios, "The Application of High-Resolution Spectral Analysis for Identifying Multiple Combined Faults in Induction Motors," IEEE Transactions on Industrial Electronics, vol. 58, no. 5, pp. 2002-2010, May 2011.

[53] A. Gandhi, T. Corrigan, and L. Parsa, "Recent Advances in Modeling and Online Detection of Stator Interturn Faults in Electrical Motors," IEEE Transactions on Industrial Electronics, vol. 58, no. 5, pp. 1564-1575, May 2011.

[54] A. M. EL-Refaie, "Fractional-Slot Concentrated-Windings Synchronous Permanent Magnet Machines: Opportunities and Challenges," IEEE Transactions on Industrial Electronics, vol. 57, no. 1, pp. 107-121, Jan. 2010.

[55] M. El Hachemi Benbouzid, "A review of induction motors signature analysis as a medium for faults detection," IEEE Transactions on Industrial Electronics, vol. 47, no. 5, pp. 984-993, 2000.

[56] B. M. Ebrahimi, J. Faiz, and M. J. Roshtkhari, "Static-, Dynamic-, and MixedEccentricity Fault Diagnoses in Permanent-Magnet Synchronous Motors," 
IEEE Transactions on Industrial Electronics, vol. 56, no. 11, pp. 4727-4739, Nov. 2009.

[57] C. H. De Angelo, G. R. Bossio, S. J. Giaccone, M. I. Valla, J. A. Solsona, and G. O. Garcia, "Online Model-Based Stator-Fault Detection and Identification in Induction Motors," IEEE Transactions on Industrial Electronics, vol. 56, no. 11, pp. 4671-4680, Nov. 2009.

[58] S. M. A. Cruz and A. J. M. Cardoso, "Multiple Reference Frames Theory: A New Method for the Diagnosis of Stator Faults in Three-Phase Induction Motors," IEEE Transactions on Energy Conversion, vol. 20, no. 3, pp. 611619, Sep. 2005.

[59] S. M. A. Cruz and A. J. M. Cardoso, "Stator winding fault diagnosis in threephase synchronous and asynchronous motors, by the extended Park's vector approach," IEEE Transactions on Industry Applications, vol. 37, no. 5, pp. 1227-1233, 2001.

[60] S. Cheng, P. Zhang, and T. G. Habetler, "An Impedance Identification Approach to Sensitive Detection and Location of Stator Turn-to-Turn Faults in a Closed-Loop Multiple-Motor Drive," IEEE Transactions on Industrial Electronics, vol. 58, no. 5, pp. 1545-1554, May 2011.

[61] F. Briz, M. W. Degner, A. Zamarron, and J. M. Guerrero, "Online stator winding fault diagnosis in inverter-fed ac machines using high-frequency signal injection," IEEE Transactions on Industry Applications, vol. 39, no. 4, pp. 1109-1117, Jul. 2003.

[62] A. Bellini, F. Filippetti, G. Franceschini, and C. Tassoni, "Closed-loop control impact on the diagnosis of induction motors faults," IEEE Transactions on Industry Applications, vol. 36, no. 5, pp. 1318-1329, 2000.

[63] M. A. Awadallah, M. M. Morcos, S. Gopalakrishnan, and T. W. Nehl, "Detection of Stator Short Circuits in VSI-Fed Brushless DC Motors Using Wavelet Transform," IEEE Transactions on Energy Conversion, vol. 21, no. 1, pp. 1-8, Mar. 2006.

[64] B. Akin, U. Orguner, H. A. Toliyat, and M. Rayner, "Low Order PWM Inverter Harmonics Contributions to the Inverter-Fed Induction Machine Fault Diagnosis," IEEE Transactions on Industrial Electronics, vol. 55, no. 2, pp. 610-619, 2008.

[65] K. Bose, "Power Electronics and Variable Frequency Drives: technology and Applications," IEEE press, pp. 9-35.

[66] B. Wu, “Advanced Drive Control Schemes,” Wiley Online Lib., pp. 285-317. 
[67] “1337-1343.pdf (application/pdf Object)." [Online]. Available: http://www.union-services.com/aevs/1337-1343.pdf. [Accessed: 23-Aug2012].

[68] M. J. Corley and R. D. Lorenz, "Rotor position and velocity estimation for a salient-pole permanent magnet synchronous machine at standstill and high speeds," IEEE Transactions on Industry Applications, vol. 34, no. 4, pp. 784789, 1998.

[69] R. Wu and G. R. Slemon, "A permanent magnet motor drive without a shaft sensor," IEEE Transactions on Industry Applications, vol. 27, no. 5, pp. 10051011, 1991.

[70] O. Wallmark, L. Harnefors, and O. Carlson, "Sensorless control of PMSM drives for hybrid electric vehicles," in 2004 IEEE 35th Annual Power Electronics Specialists Conference (IEEE Cat. No.04CH37551), pp. 40174023.

[71] D. M. Vilathgamuwa and M. A. Rahman, "Implementation of an artificialneural-network-based real-time adaptive controller for an interior permanentmagnet motor drive," IEEE Transactions on Industry Applications, vol. 39, no. 1, pp. 96-104, Jan. 2003.

[72] V. Utkin, "Sliding mode observers for electric machines-an overview," in IEEE 2002 28th Annual Conference of the Industrial Electronics Society. IECON 02, vol. 3, pp. 1842-1847.

[73] S. Shinnaka, "New 'D-State-Observer'-Based Vector Control for Sensorless Drive of Permanent-Magnet Synchronous Motors," IEEE Transactions on Industry Applications, vol. 41, no. 3, pp. 825-833, May 2005.

[74] M. Rashed, P. F. A. MacConnell, A. F. Stronach, and P. Acarnley, "Sensorless Indirect-Rotor-Field-Orientation Speed Control of a Permanent-Magnet Synchronous Motor With Stator-Resistance Estimation," IEEE Transactions on Industrial Electronics, vol. 54, no. 3, pp. 1664-1675, Jun. 2007.

[75] S. Chi, L. Xu, and Z. Zhang, "Sliding Mode Sensorless Control of PM Synchronous Motor for Direct-Driven Washing Machines," in Conference Record of the 2006 IEEE Industry Applications Conference Forty-First IAS Annual Meeting, 2006, vol. 2, pp. 873-879.

[76] F. Briz, M. W. Degner, P. Garcia, and R. D. Lorenz, "Comparison of SaliencyBased Sensorless Control Techniques for AC Machines," IEEE Transactions on Industry Applications, vol. 40, no. 4, pp. 1107-1115, Jul. 2004.

[77] F. Briz, M. W. Degner, P. Garcia, and R. D. Lorenz, "Comparison of SaliencyBased Sensorless Control Techniques for AC Machines," IEEE Transactions on Industry Applications, vol. 40, no. 4, pp. 1107-1115, Jul. 2004. 
[78] S. Bolognani, R. Oboe, and M. Zigliotto, "Sensorless full-digital PMSM drive with EKF estimation of speed and rotor position," IEEE Transactions on Industrial Electronics, vol. 46, no. 1, pp. 184-191, 1999.

[79] K. J. Astrom and B. Wittenmark, "A survey of adaptive control applications," in Proceedings of 1995 34th IEEE Conference on Decision and Control, vol. 1, pp. 649-654.

[80] A. M. EL-Refaie and T. M. Jahns, "Comparison of synchronous PM machine types for wide constant-power speed range operation," in Fourtieth IAS Annual Meeting. Conference Record of the 2005 Industry Applications Conference, 2005., vol. 2, pp. 1015-1022.

[81] P. B. Reddy, A. El-Refaie, K.-K. Huh, J. K. Tangudu, and T. M. Jahns, "Comparison of interior and surface PM machines equipped with fractionalslot concentrated windings for hybrid traction applications," in 2011 IEEE Energy Conversion Congress and Exposition, 2011, pp. 2252-2259.

[82] "Brushless permanent-magnet motor design." [Online]. Available: http://books.google.com/books/about/Brushless_permanent_magnet_motor_des ign.html?id=PuhSAAAAMAAJ. [Accessed: 23-Aug-2012].

[83] “Analysis of Electric Machinery and Drive Systems," pp. 1-65.

[84] Z. Q. Zhu, S. Ruangsinchaiwanich, and D. Howe, "Synthesis of cogging-torque waveform from analysis of a single stator slot," IEEE Transactions on Industry Applications, vol. 42, no. 3, pp. 650-657, May 2006.

[85] Z. Q. Zhu and D. Howe, "Influence of design parameters on cogging torque in permanent magnet machines," IEEE Transactions on Energy Conversion, vol. 15 , no. 4, pp. 407-412, 2000.

[86] A. H. Wijenayake, J. M. Bailey, and P. J. McCleer, "Design optimization of an axial gap permanent magnet brushless DC motor for electric vehicle applications," in IAS '95. Conference Record of the 1995 IEEE Industry Applications Conference Thirtieth IAS Annual Meeting, vol. 1, pp. 685-692.

[87] P. R. Upadhyay and K. R. Rajagopal, Genetic algorithm based design optimization of a permanent magnet brushless dc motor, vol. 97. 2005.

[88] R. F. Schiferl and T. A. Lipo, "Power capability of salient pole permanent magnet synchronous motors in variable speed drive applications," IEEE Transactions on Industry Applications, vol. 26, no. 1, pp. 115-123, 1990.

[89] S. Russenschuck, "Application of Lagrange multiplier estimation to the design optimization of permanent magnet synchronous machines," IEEE Transactions on Magnetics, vol. 28, no. 2, pp. 1525-1528, Mar. 1992. 
[90] S. Russenschuck, "Mathematical optimization techniques for the design of permanent magnet synchronous machines based on numerical field calculation," IEEE Transactions on Magnetics, vol. 26, no. 2, pp. 638-641, Mar. 1990.

[91] P. B. Reddy, A. M. El-Refaie, K.-K. Huh, J. K. Tangudu, and T. M. Jahns, "Comparison of Interior and Surface PM Machines Equipped With FractionalSlot Concentrated Windings for Hybrid Traction Applications," IEEE Transactions on Energy Conversion, vol. 27, no. 3, pp. 593-602, Sep. 2012.

[92] A. B. Proca, A. Keyhani, and A. EL-Antably, "Analytical model for permanent magnet motors with surface mounted magnets," IEEE Transactions on Energy Conversion, vol. 18, no. 3, pp. 386-391, Sep. 2003.

[93] D. Pavlik, V. K. Garg, J. R. Repp, and J. Weiss, "A finite element technique for calculating the magnet sizes and inductances of permanent magnet machines," vol. 3, 1988.

[94] M. Olaru, M. I. McGelp, T. J. E. Miller, J. H. Davies, and K. F. Rasmussen, "Analytical and numerical computation of air-gap magnetic fields in brushless motors with surface permanent magnets," IEEE Transactions on Industry Applications, vol. 36, no. 6, pp. 1547-1554, 2000.

[95] F. Magnussen, P. Thelin, and C. Sadarangani, "Performance evaluation of permanent magnet synchronous machines with concentrated and distributed windings including the effect of field-weakening," vol. 2. pp. 679-685, 2004.

[96] T. Lubin, S. Mezani, and A. Rezzoug, "2-D Exact Analytical Model for Surface-Mounted Permanent-Magnet Motors With Semi-Closed Slots," IEEE Transactions on Magnetics, vol. 47, no. 2, pp. 479-492, Feb. 2011.

[97] T. M. Jahns, "Component rating requirements for wide constant power operation of interior PM synchronous machine drives," in Conference Record of the 2000 IEEE Industry Applications Conference. Thirty-Fifth IAS Annual Meeting and World Conference on Industrial Applications of Electrical Energy (Cat. No.00CH37129), vol. 3, pp. 1697-1704.

[98] D. C. Hanselman, "Minimum torque ripple, maximum efficiency excitation of brushless permanent magnet motors," IEEE Transactions on Industrial Electronics, vol. 41, no. 3, pp. 292-300, Jun. 1994.

[99] A. M. El-Refaie, T. M. Jahns, and D. W. Novotny, "Analysis of Surface Permanent Magnet Machines With Fractional-Slot Concentrated Windings," IEEE Transactions on Energy Conversion, vol. 21, no. 1, pp. 34-43, Mar. 2006.

[100] A. M. EL-Refaie, "Fractional-Slot Concentrated-Windings Synchronous Permanent Magnet Machines: Opportunities and Challenges," IEEE Transactions on Industrial Electronics, vol. 57, no. 1, pp. 107-121, Jan. 2010. 
[101] L. Dosiek and P. Pillay, "Cogging Torque Reduction in Permanent Magnet Machines," IEEE Transactions on Industry Applications, vol. 43, no. 6, pp. 1565-1571, 2007.

[102] A. Chen, R. Nilssen, and A. Nysveen, "Harmonic analysis and comparison of the back EMFs of four permanent magnet machine with different winding arrangements."

[103] C. Breton, J. Bartolome, J. A. Benito, G. Tassinario, I. Flotats, C. W. Lu, and B. J. Chalmers, "Influence of machine symmetry on reduction of cogging torque in permanent-magnet brushless motors," IEEE Transactions on Magnetics, vol. 36, no. 5, pp. 3819-3823, 2000.

[104] N. Boules, "Design optimization of permanent magnet DC motors," IEEE Transactions on Industry Applications, vol. 26, no. 4, pp. 786-792, 1990.

[105] K. Boughrara, B. L. Chikouche, R. Ibtiouen, D. Zarko, and O. Touhami, "Analytical Model of Slotted Air-Gap Surface Mounted Permanent-Magnet Synchronous Motor With Magnet Bars Magnetized in the Shifting Direction," IEEE Transactions on Magnetics, vol. 45, no. 2, pp. 747-758, Feb. 2009.

[106] A. K. Adnanes, "Torque analysis of permanent magnet synchronous motors," in PESC '91 Record 22nd Annual IEEE Power Electronics Specialists Conference, pp. 695-701.

[107] W. Yang, P. J. Tavner, and M. R. Wilkinson, "Condition monitoring and fault diagnosis of a wind turbine synchronous generator drive train," IET Renewable Power Generation, vol. 3, no. 1, p. 1, 2009.

[108] P. J. Tavner, "Review of condition monitoring of rotating electrical machines," IET Electric Power Applications, vol. 2, no. 4, p. 215, 2008.

[109] R. Krishnan, A. S. Bharadwaj, and P. N. Materu, "Computer-aided design of electrical machines for variable speed applications," IEEE Transactions on Industrial Electronics, vol. 35, no. 4, pp. 560-571, 1988.

[110] P. M. Menghal and A. J. Laxmi, "Real time control of electrical machine drives: A review," in 2010 International Conference on Power, Control and Embedded Systems, 2010, pp. 1-6.

[111] "Computer-Aided Design of Electric Machinery (Monographs in Modern Electrical Technology): 9780262220163 - Book Smart.” [Online]. Available: http://www.abebooks.com/Computer-Aided-Design-Electric-MachineryMonographs-Modern/4466684218/bd. [Accessed: 25-Aug-2012].

[112] T. Dreher, R. Perrin-Bit, G. Meunier, and J. L. Coulomb, “A three dimensional finite element modelling of rotating machines involving movement and external circuit," IEEE Transactions on Magnetics, vol. 32, no. 3, pp. 1070 1073, May 1996. 
[113] K. Hameyer, J. Driesen, H. De Gersem, and R. Belmans, "The classification of coupled field problems," IEEE Transactions on Magnetics, vol. 35, no. 3, pp. 1618-1621, May 1999.

[114] N. M. Abe, J. R. Cardoso, and A. Foggia, "Coupling electric circuit and 2DFEM model with Dommel's approach for transient analysis [of EM devices]," IEEE Transactions on Magnetics, vol. 34, no. 5, pp. 3487-3490, 1998.

[115] T. Dreher and G. Meunier, "3D line current model of coils and external circuits," IEEE Transactions on Magnetics, vol. 31, no. 3, pp. 1853-1856, May 1995.

[116] J. Gilmore, Z. Badics, and Z. J. Cendes, "Finite element analysis of induction motors based on computing detailed equivalent circuit parameters," IEEE Transactions on Magnetics, vol. 34, no. 5, pp. 3499-3502, 1998.

[117] H. Choi, I. Park, and S.-H. Lee, "Electromagnetic body force calculation based on virtual air gap," Journal of Applied Physics, vol. 99, no. 8, p. 08H903, 2006.

[118] Z. Ren and A. Razek, "Force calculation by Maxwell stress tensor in 3D hybrid finite element-boundary integral formulation," IEEE Transactions on Magnetics, vol. 26, no. 5, pp. 2774-2776, 1990.

[119] E. A. Aronson and J. R. Brauer, "Magnetic torque or force calculation by direct differentiation of finite element coenergy," IEEE Transactions on Magnetics, vol. 25 , no. 5 , pp. 3578-3580, 1989.

[120] C. M. Arturi, "Electromagnetic force calculations on a 3-phase autotransformer under time-varying fault by a 3D non-linear finite element code," IEEE Transactions on Magnetics, vol. 29, no. 2, pp. 2010-2013, Mar. 1993.

[121] E. A. Aronson and J. R. Brauer, "Magnetic torque or force calculation by direct differentiation of finite element coenergy," IEEE Transactions on Magnetics, vol. 25, no. 5, pp. 3578-3580, 1989.

[122] C. M. Arturi, "Electromagnetic force calculations on a 3-phase autotransformer under time-varying fault by a 3D non-linear finite element code," IEEE Transactions on Magnetics, vol. 29, no. 2, pp. 2010-2013, Mar. 1993.

[123] Z. Ren and A. Razek, "Force calculation by Maxwell stress tensor in 3D hybrid finite element-boundary integral formulation," IEEE Transactions on Magnetics, vol. 26, no. 5, pp. 2774-2776, 1990.

[124] P. J. Leonard and D. Rodger, "Modelling voltage forced coils using the reduced scalar potential method," IEEE Transactions on Magnetics, vol. 28, no. 2, pp. 1615-1618, Mar. 1992.

[125] C. M. Arturi, "Electromagnetic Force Calculations on a 3-Phase Autotransformer Under Time-Varying Fault by a 3D Non-Linear Finite 
Element Code," in Digest of the Fifth Biennial IEEE Conference on Electromagnetic Field Computation, pp. WOA6-WOA6.

[126] E. A. Aronson and J. R. Brauer, "Magnetic torque or force calculation by direct differentiation of finite element coenergy," IEEE Transactions on Magnetics, vol. 25, no. 5, pp. 3578-3580, 1989.

[127] J. A. Walker, D. G. Dorrell, and C. Cossar, "Flux-linkage calculation in permanent-magnet motors using the frozen permeabilities method," IEEE Transactions on Magnetics, vol. 41, no. 10, pp. 3946-3948, Oct. 2005.

[128] H. Hong and J. Yoo, "Shape Design of the Surface Mounted Permanent Magnet in a Synchronous Machine," IEEE Transactions on Magnetics, vol. 47, no. 8, pp. 2109-2117, Aug. 2011.

[129] P. Virtic, P. Pisek, T. Marcic, M. Hadziselimovic, and B. Stumberger, "Analytical Analysis of Magnetic Field and Back Electromotive Force Calculation of an Axial-Flux Permanent Magnet Synchronous Generator With Coreless Stator," IEEE Transactions on Magnetics, vol. 44, no. 11, pp. 43334336, Nov. 2008.

[130] L. Chang, "An improved FE inductance calculation for electrical machines," IEEE Transactions on Magnetics, vol. 32, no. 4, pp. 3237-3245, Jul. 1996.

[131] T. J. E. Miller, M. I. McGilp, D. A. Staton, and J. J. Bremner, "Calculation of inductance in permanent-magnet DC motors," IEE Proceedings - Electric Power Applications, vol. 146, no. 2, p. 129, 1999.

[132] R. S. Mamak and E. R. Laithwaite, "Numerical evaluation of inductance and a.c. resistance, with particular reference to electrical machines," Proceedings of the IEE Part C: Monographs, vol. 108, no. 13, p. 252, 1961.

[133] J.-B. Dastous, "Nonlinear finite-element analysis of stranded conductors with variable bending stiffness using the tangent stiffness method," vol. 20, 2005.

[134] A. Gagnoud and B. Dumont, "3D finite element method with impedance boundary condition for the modeling of molten metal shape in electromagnetic casting," IEEE Transactions on Magnetics, vol. 36, no. 4, pp. 1329-1332, Jul. 2000 .

[135] C. Pes, J. M. Baze, C. Berriaud, L. Chevalier, F. P. Juster, W. Kozanecki, and P. Vedrine, "Lorenz Forces Exerted by the Magnetic Mirror and Magnetic Influence of the Cryostat on the ATLAS BT Coils During the Test," IEEE Transactions on Applied Superconductivity, vol. 18, no. 2, pp. 411-414, Jun. 2008 .

[136] J. C. H. Bone, "Cooling and cooling circuits for electric motors," vol. 1, 1978. 
[137] T. A. Jankowski, F. C. Prenger, D. D. Hill, S. R. O’Bryan, K. K. Sheth, E. B. Brookbank, D. F. A. Hunt, and Y. A. Orrego, "Development and Validation of a Thermal Model for Electric Induction Motors," IEEE Transactions on Industrial Electronics, vol. 57, no. 12, pp. 4043-4054, Dec. 2010.

[138] G. D. Demetriades, H. Z. de la Parra, E. Andersson, and H. Olsson, "A RealTime Thermal Model of a Permanent-Magnet Synchronous Motor," IEEE Transactions on Power Electronics, vol. 25, no. 2, pp. 463-474, Feb. 2010.

[139] C. Kral, A. Haumer, M. Haigis, H. Lang, and H. Kapeller, "Comparison of a CFD Analysis and a Thermal Equivalent Circuit Model of a TEFC Induction Machine With Measurements," IEEE Transactions on Energy Conversion, vol. 24, no. 4, pp. 809-818, Dec. 2009.

[140] A. M. EL-Refaie, N. C. Harris, T. M. Jahns, and K. M. Rahman, "Thermal Analysis of Multibarrier Interior PM Synchronous Machine Using Lumped Parameter Model," IEEE Transactions on Energy Conversion, vol. 19, no. 2, pp. 303-309, Jun. 2004.

[141] O. A. Mohammed, S. Liu, and Z. Liu, "FE-based physical phase variable models of electric machines and transformers for dynamic simulations," in 2006 IEEE Power Engineering Society General Meeting, 2006, p. 7 pp.

[142] O. A. Mohammed, S. Liu, and Z. Liu, "Physical modeling of PM synchronous motors for integrated coupling with Machine drives," IEEE Transactions on Magnetics, vol. 41, no. 5, pp. 1628-1631, May 2005.

[143] O. A. Mohammed, S. Liu, and Z. Liu, "A phase variable PM machine model for integrated motor drive systems," in 2004 IEEE 35th Annual Power Electronics Specialists Conference (IEEE Cat. No.04CH37551), pp. 48254831 .

[144] S. Liu, O. A. Mohammed, and Z. Liu, "An Improved FE-Based Phase Variable Model of PM Synchronous Machines Including Dynamic Core Losses," IEEE Transactions on Magnetics, vol. 43, no. 4, pp. 1801-1804, Apr. 2007.

[145] O. A. Mohammed, S. Liu, and Z. Liu, "Phase-variable model of PM synchronous machines for integrated motor drives," IEE Proceedings - Science, Measurement and Technology, vol. 151, no. 6, p. 423, 2004.

[146] O. A. Mohammed, S. Liu, Z. Liu, and N. Abed, "Physical phase variable models of electrical equipments and their applications in integrated drive simulation for shipboard power system," in IEEE Electric Ship Technologies Symposium, 2005., pp. 163-170.

[147] O. A. Mohammed, S. Liu, and Z. Liu, "An FE-based Physical Phase Variable Model for PM Synchronous Machines Including Dynamic Core Losses," in 2006 12th Biennial IEEE Conference on Electromagnetic Field Computation, pp. 137-137. 
[148] O. A. Mohammed, Z. Liu, S. Liu, and N. Y. Abed, "Internal Short Circuit Fault Diagnosis for PM Machines Using FE-Based Phase Variable Model and Wavelets Analysis," IEEE Transactions on Magnetics, vol. 43, no. 4, pp. 17291732, Apr. 2007.

[149] J. A. Tapia, F. Leonardi, and T. A. Lipo, "Consequent-pole permanent-magnet machine with extended field-weakening capability," IEEE Transactions on Industry Applications, vol. 39, no. 6, pp. 1704-1709, Nov. 2003.

[150] W. L. Soong, "Field-weakening performance of brushless synchronous AC motor drives," IEE Proceedings - Electric Power Applications, vol. 141, no. 6, p. 331, 1994.

[151] R. Dhaouadi and N. Mohan, "Analysis of current-regulated voltage-source inverters for permanent magnet synchronous motor drives in normal and extended speed ranges," IEEE Transactions on Energy Conversion, vol. 5, no. 1, pp. 137-144, Mar. 1990.

[152] J.-M. Kim and S.-K. Sul, "Speed control of interior permanent magnet synchronous motor drive for the flux weakening operation," IEEE Transactions on Industry Applications, vol. 33, no. 1, pp. 43-48, 1997.

[153] Y. S. Kim, Y. K. Choi, and J. H. Lee, "Speed-sensorless vector control for permanent-magnet synchronous motors based on instantaneous reactive power in the wide-speed region," IEE Proceedings - Electric Power Applications, vol. 152 , no. 5 , p. $1343,2005$.

[154] S. R. Macminn and T. M. Jahns, "Control techniques for improved high-speed performance of interior PM synchronous motor drives," IEEE Transactions on Industry Applications, vol. 27, no. 5, pp. 997-1004, 1991.

[155] S. Morimoto, M. Sanada, and Y. Takeda, "Wide-speed operation of interior permanent magnet synchronous motors with high-performance current regulator," IEEE Transactions on Industry Applications, vol. 30, no. 4, pp. 920-926, 1994.

[156] S. Morimoto, Y. Takeda, T. Hirasa, and K. Taniguchi, "Expansion of operating limits for permanent magnet motor by current vector control considering inverter capacity," IEEE Transactions on Industry Applications, vol. 26, no. 5, pp. 866-871, 1990.

[157] J.-H. Song, J.-M. Kim, and S.-K. Sul, "A new robust SPMSM control to parameter variations in flux weakening region," in Proceedings of the 1996 IEEE IECON. 22nd International Conference on Industrial Electronics, Control, and Instrumentation, vol. 2, pp. 1193-1198.

[158] S. D. Sudhoff, K. A. Corzine, and H. J. Hegner, "A flux-weakening strategy for current-regulated surface-mounted permanent-magnet machine drives," IEEE Transactions on Energy Conversion, vol. 10, no. 3, pp. 431-437, 1995. 
[159] S. Chi, "Position-sensorless control of permanent magnet synchronous machines over wide speed range," 2007.

[160] F. Briz, M. W. Degner, P. Garcia, and R. D. Lorenz, "Comparison of SaliencyBased Sensorless Control Techniques for AC Machines," IEEE Transactions on Industry Applications, vol. 40, no. 4, pp. 1107-1115, Jul. 2004.

[161] M. Schroedl, "Sensorless control of AC machines at low speed and standstill based on the 'INFORM' method," in IAS '96. Conference Record of the 1996 IEEE Industry Applications Conference Thirty-First IAS Annual Meeting, vol. 1, pp. 270-277.

[162] R. Wu and G. R. Slemon, "A permanent magnet motor drive without a shaft sensor," IEEE Transactions on Industry Applications, vol. 27, no. 5, pp. 10051011, 1991.

[163] J.-H. Jang, J.-I. Ha, M. Ohto, K. Ide, and S.-K. Sul, “Analysis of PermanentMagnet Machine for Sensorless Control Based on High-Frequency Signal Injection," IEEE Transactions on Industry Applications, vol. 40, no. 6, pp. 1595-1604, Nov. 2004.

[164] M. Rashed, P. F. A. MacConnell, A. F. Stronach, and P. Acarnley, "Sensorless Indirect-Rotor-Field-Orientation Speed Control of a Permanent-Magnet Synchronous Motor With Stator-Resistance Estimation," IEEE Transactions on Industrial Electronics, vol. 54, no. 3, pp. 1664-1675, Jun. 2007.

[165] S. Shinnaka, “New 'D-State-Observer'-Based Vector Control for Sensorless Drive of Permanent-Magnet Synchronous Motors," IEEE Transactions on Industry Applications, vol. 41, no. 3, pp. 825-833, May 2005.

[166] K. J. Astrom and B. Wittenmark, "A survey of adaptive control applications," in Proceedings of 1995 34th IEEE Conference on Decision and Control, vol. 1, pp. 649-654.

[167] D. M. Vilathgamuwa and M. A. Rahman, "Implementation of an artificialneural-network-based real-time adaptive controller for an interior permanentmagnet motor drive," IEEE Transactions on Industry Applications, vol. 39, no. 1, pp. 96-104, Jan. 2003.

[168] M. J. Corley and R. D. Lorenz, "Rotor position and velocity estimation for a salient-pole permanent magnet synchronous machine at standstill and high speeds," IEEE Transactions on Industry Applications, vol. 34, no. 4, pp. 784 789, 1998.

[169] O. Wallmark, L. Harnefors, and O. Carlson, "Sensorless control of PMSM drives for hybrid electric vehicles," in 2004 IEEE 35th Annual Power Electronics Specialists Conference (IEEE Cat. No.04CH37551), pp. 40174023. 
[170] D. P. A. D. Xue, Y. Chen, Linear feedback control analysis and design with MATLAB. Society for Industrial and Applied Mathematics, 2009, pp. 92-98.

[171] and R. G. H. R. M. Tallam, T. G. Habetler, "Stator winding turn-fault detection for closed-loop induction motor drives," IEEE Trans. Industry Applications, vol. 39, pp. 720-724, 2003.

[172] D. Howe and G. Jewell, "Analytical Prediction of the Short-Circuit Current in Fault-Tolerant Permanent-Magnet Machines," IEEE Transactions on Industrial Electronics, vol. 55, no. 12, pp. 4210-4217, Dec. 2008.

[173] M. El Hachemi Benbouzid, "A review of induction motors signature analysis as a medium for faults detection," IEEE Transactions on Industrial Electronics, vol. 47, no. 5, pp. 984-993, 2000.

[174] B. Akin, S. Choi, U. Orguner, and H. A. Toliyat, "A Simple Real-Time Fault Signature Monitoring Tool for Motor-Drive-Embedded Fault Diagnosis Systems," IEEE Transactions on Industrial Electronics, vol. 58, no. 5, pp. 1990-2001, May 2011.

[175] R. M. Tallam and T. G. Habetler, "A robust, on-line turn-fault detection technique for induction machines based on monitoring the sequence component impedance matrix," IEEE Transactions on Power Electronics, vol. 18, no. 3, pp. 865-872, May 2003.

[176] A. Bellini, F. Filippetti, G. Franceschini, and C. Tassoni, "Closed-loop control impact on the diagnosis of induction motors faults," IEEE Transactions on Industry Applications, vol. 36, no. 5, pp. 1318-1329, 2000.

[177] S. Cheng, P. Zhang, and T. G. Habetler, "An Impedance Identification Approach to Sensitive Detection and Location of Stator Turn-to-Turn Faults in a Closed-Loop Multiple-Motor Drive," IEEE Transactions on Industrial Electronics, vol. 58, no. 5, pp. 1545-1554, May 2011.

[178] B. Akin, U. Orguner, H. A. Toliyat, and M. Rayner, "Low Order PWM Inverter Harmonics Contributions to the Inverter-Fed Induction Machine Fault Diagnosis," IEEE Transactions on Industrial Electronics, vol. 55, no. 2, pp. 610-619, 2008.

[179] A. Garcia-Perez, R. de J. Romero-Troncoso, E. Cabal-Yepez, and R. A. Osornio-Rios, "The Application of High-Resolution Spectral Analysis for Identifying Multiple Combined Faults in Induction Motors," IEEE Transactions on Industrial Electronics, vol. 58, no. 5, pp. 2002-2010, May 2011.

[180] J. Pons-Llinares, J. A. Antonino-Daviu, M. Riera-Guasp, M. Pineda-Sanchez, and V. Climente-Alarcon, "Induction Motor Diagnosis Based on a Transient Current Analytic Wavelet Transform via Frequency B-Splines," IEEE 
Transactions on Industrial Electronics, vol. 58, no. 5, pp. 1530-1544, May 2011.

[181] A. Gandhi, T. Corrigan, and L. Parsa, "Recent Advances in Modeling and Online Detection of Stator Interturn Faults in Electrical Motors," IEEE Transactions on Industrial Electronics, vol. 58, no. 5, pp. 1564-1575, May 2011 .

[182] M. A. Awadallah, M. M. Morcos, S. Gopalakrishnan, and T. W. Nehl, "Detection of Stator Short Circuits in VSI-Fed Brushless DC Motors Using Wavelet Transform," IEEE Transactions on Energy Conversion, vol. 21, no. 1, pp. 1-8, Mar. 2006.

[183] C. Bianchini, E. Fornasiero, T. N. Matzen, N. Bianchi, and A. Bellini, "Fault detection of a five-phase Permanent-Magnet machine," in 2008 34th Annual Conference of IEEE Industrial Electronics, 2008, pp. 1200-1205.

[184] F. Briz, M. W. Degner, A. Zamarron, and J. M. Guerrero, "Online stator winding fault diagnosis in inverter-fed ac machines using high-frequency signal injection," IEEE Transactions on Industry Applications, vol. 39, no. 4, pp. 1109-1117, Jul. 2003.

[185] S. M. A. Cruz and A. J. M. Cardoso, "Multiple Reference Frames Theory: A New Method for the Diagnosis of Stator Faults in Three-Phase Induction Motors," IEEE Transactions on Energy Conversion, vol. 20, no. 3, pp. 611619, Sep. 2005.

[186] H. Henao, C. Demian, and G.-A. Capolino, "A frequency-domain detection of stator winding faults in induction machines using an external flux sensor," IEEE Transactions on Industry Applications, vol. 39, no. 5, pp. 1272-1279, Sep. 2003.

[187] Y. Lee and T. G. Habetler, "An On-Line Stator Turn Fault Detection Method for Interior PM Synchronous Motor Drives," in APEC 07 - Twenty-Second Annual IEEE Applied Power Electronics Conference and Exposition, 2007, pp. $825-831$.

[188] P. Neti and S. Nandi, "Stator Inter-turn Fault Detection of Synchronous Machines Using Field Current Signature Analysis," in Conference Record of the 2006 IEEE Industry Applications Conference Forty-First IAS Annual Meeting, 2006, vol. 5, pp. 2360-2367.

[189] B. Vaseghi, N. Takorabet, and F. Meibody-Tabar, "Fault Analysis and Parameter Identification of Permanent-Magnet Motors by the Finite-Element Method," IEEE Transactions on Magnetics, vol. 45, no. 9, pp. 3290-3295, Sep. 2009.

[190] T. M. Wolbank, K. A. Loparo, and R. Wohrnschimmel, "Inverter statistics for online detection of stator asymmetries in inverter-fed induction motors," IEEE 
Transactions on Industry Applications, vol. 39, no. 4, pp. 1102-1108, Jul. 2003.

[191] C. S. Kallesoe, R. Izadi-Zamanabadi, P. Vadstrup, and H. Rasmussen, "Observer-Based Estimation of Stator-Winding Faults in Delta-Connected Induction Motors: A Linear Matrix Inequality Approach," IEEE Transactions on Industry Applications, vol. 43, no. 4, pp. 1022-1031, 2007.

[192] N. Leboeuf, T. Boileau, B. Nahid-Mobarakeh, and F. Meibody-Tabar, "Fault Detection in a Current Controlled PM Drive Using Back-EMF Estimation and Residual Analysis," in 2010 IEEE Industry Applications Society Annual Meeting, 2010, pp. 1-6.

[193] N. Leboeuf, "Fault detection in a current controlled PM drive using back EMF estimation and residual analysis," IAS2010, PP. 1-6, 2010.

[194] O. A. Mohammed, D. A. Lowther, M. H. Lean, and B. Alhalabi, "On the creation of a generalized design optimization environment for electromagnetic devices," IEEE Transactions on Magnetics, vol. 37, no. 5, pp. 3562-3565, 2001.

[195] J. Ouyang and D. A. Lowther, "The use of semantic networks to adapt a design prototype for electromagnetic device optimization," in Digests of the 2010 14th Biennial IEEE Conference on Electromagnetic Field Computation, 2010, pp. $1-1$.

[196] M. Shan, J. Wu, and D. Peng, "Particle Swarm and Ant Colony Algorithms Hybridized for Multi-Mode Resource-constrained Project Scheduling Problem with Minimum Time Lag," in 2007 International Conference on Wireless Communications, Networking and Mobile Computing, 2007, pp. 5893-5897.

[197] K. E. Parsopoulos and M. N. Vrahatis, "On the Computation of All Global Minimizers Through Particle Swarm Optimization," IEEE Transactions on Evolutionary Computation, vol. 8, no. 3, pp. 211-224, Jun. 2004.

[198] Z.-H. Zhan, J. Zhang, Y. Li, and H. S.-H. Chung, "Adaptive particle swarm optimization.," IEEE transactions on systems, man, and cybernetics. Part B, Cybernetics: a publication of the IEEE Systems, Man, and Cybernetics Society, vol. 39 , no. 6 , pp. 1362-81, Dec. 2009.

[199] A. E. Smith, "Multi-objective optimization using evolutionary algorithms [Book Review]," IEEE Transactions on Evolutionary Computation, vol. 6, no. 5, pp. 526-526, Oct. 2002.

[200] G. Y. Sizov, D. M. Ionel, and N. A. O. Demerdash, "Modeling and Parametric Design of Permanent-Magnet AC Machines Using Computationally Efficient Finite-Element Analysis," IEEE Transactions on Industrial Electronics, vol. 59, no. 6, pp. 2403-2413, Jun. 2012. 
[201] Z. Liu and O. A. Mohammed, "Equivalent Hardware Representation of PM Synchronous Motors From the Physics-Based Phase Variable Model Obtained Through FE Computation," IEEE Transactions on Magnetics, vol. 45, no. 3, pp. 1450-1453, Mar. 2009.

[202] D. K. Cheng, Field and Wave Electromagnetics (2nd Edition). AddisonWesley, 1989, p. 717. 


\section{VITA}

\section{ALI SARIKHANI}

August 9, $1981 \quad$ Born, Shiraz, Iran

2000-2005

B.Sc. Power and Water University of Technology

$2005-2008$

M.Sc. Shahrood University of Technology

2008-2009

Lecturer, Azad University, Iran

2009-2011

Research Assistant, Florida International University

$2011-2012$

Graduate Assistant, Florida International University

$2012-2013$

Dissertation Year Fellowship, Florida International University

\section{SELECTED PUBLICATION AND PRESENTATIONS}

- Ali Sarikhani, Osama. A. Mohammed, "HIL-based finite-element design optimization process for the computational prototyping of electric motor-drives," IEEE Transactions on Energy Conversion, Vol. 27, Issue 3, pp.737-746, DOI: 10.1109/TEC.2012.2200897,2012.

- Ali Sarikhani, Osama Mohammed "Sensorless Control of PM Synchronous Machines by Hybrid Physics-based EMF Observer," IEEE Transactions on Energy conversion, Issue 99, pp.1-9, July 2012, DOI 10.1109/TEC.2012.2208646.

- Ali Sarikhani, Osama Mohammed, "Inter-turn Fault Detection in PM Synchronous Machines by Physics-based EMF Estimation," IEEE Transactions on industrial Electronic, 12-TIE-0597, April 2012.

- Ali Sarikhani, Osama Mohammed "Demagnetization control for reliable flux weakening control in PM synchronous machine," IEEE Transactions on Energy Conversion, TEC-00225-2012, May 2012.

- Ali Sarikhani, O. Mohammed, "Multi-objective design optimization of coupled PM synchronous motor-drive using physics-based modeling approach," IEEE Transactions on Magnetics, Vol.47, pp. 1266-1269, May 2011.

- Ali Sarikhani, M. Barzegaran, O. A. Mohammed, "Optimum Equivalent Models of Multi-source Systems for the Study of Electromagnetic Signatures and radiated Emissions from Electric Drives," IEEE Transactions on Magnetics, Vol. 48, Issue 2, pp. 1011-1014, 2012.

- Ali Sarikhani and O. A. Mohammed, "Electromagnetic field computation with external circuit coupling for evaluating the performance of electric motor 
designs," Journal of Applied Computational Electromagnetics, Vol. 26, No.12 ,Dec. 2011.

- Andrew Rosales, Ali Sarikhani, O. A. Mohammed, "Evaluation of radiated EMI due to high frequency switching in pulse width modulated motor drives," IEEE Transactions on Magnetics, Vol. 47, pp. 1474-1477, May 2011.

- Ali Sarikhani, Arash Nejadpak, Osama Mohammed, "Coupled Field-Circuit Estimation of Operational Inductance in PM Synchronous Machines by a Realtime Physics-based Inductance Observer," CEFC2012.

- Ali Sarikhani, O. A. Mohammed "Demagnetization Control for Reliable Flux Weakening Control in PM Synchronous Machine," presented at IAS2012 Annual meeting, Submission ID: 34, 2012.

- Ali Sarikhani, O. A. Mohammed, "Real-time Demagnetization Assessment of PM Synchronous Machine,” presented at ICEM2012, Paper ID: FD-011584, 2012.

- Ali Sarikhani, Osama Mohammed, "Inter-turn Fault Detection in PM Synchronous Machines by Physics-based EMF Estimation," presented at ECCE2012, paper ID: 1338, ECCE2012.

- Ali Sarikhani, O. A. Mohammed, "Design of Physics-based EMF Observer for Sensorless Control of PM Synchronous Machines," presented at PES2012 general meeting, paper No.2012GM1010, 2012.

- O. A. Mohammed, Andrew Rosales, Ali Sarikhani, "Evaluation of Radiated EMI Due to High Frequency Switching in Pulse Width Modulated Motor Drives," Presented at IEEE Conf. on Electromagnetic Field Computations CEFC2010, PP. 1, DOI: 10.1109/CEFC.2010.5481770, 2010.

- Nejadpak, M. R. Barzegaran, Ali Sarikhani , O. A. Mohammed, "Design of Planar Inductor Based Z-source Inverter for Residential Alternate Energy Sources," proc. of 26th annual IEEE Conf. on Applied power Electronic, APEC2011, pp. 1698-1703, DOI: .10.1109/APEC.2011.5744824, 2011.

- Ali Sarikhani, Wilder Saint-Hilaire and Osama A. Mohammed, "Design Improvement of Coupled IPM Motor-Drive using Physics-Based Motor Model and Evolutionary Approaches," Presented at IEEE Conf. on Energy Conversion Congress and expositions, ECCE2010, pp. 4115-4122, DOI: 10.1109/ECCE.2010.5617752, 2010.

- Ali Sarikhani, Osama A. Mohammed, "Inter-turn Fault Modeling of a Variable Speed PM Wind Generator Using Physics-based Approach," Presented at IEEE International conf. on machines and drives, pp. 646-651, may 2011.

- Ali Sarikhani, Osama A. Mohammed, "Development of Transient FE-PhysicsBased Model of Induction for Real Time Integrated Drive Simulations" presented at proc. of IEEE proc. international conf. on machines and drives, pp. 646-651, May 2011. 
- Ali Sarikhani, Behrooz Mirafzal, Osama Mohammed, "Inter-Turn Fault Diagnosis of PM Synchronous Generator for Variable Speed Wind Applications Using Floating-Space-Vector," Presented at IEEE 36th Annual Conf. on Industrial Electronic Society IECON2010, pp. 2628-2633, DOI: 10.1109/IECON.2010.5675137, 2010.

- Ali Sarikhani, and O. A. Mohammed, "Fast Inter-Turn Fault Modeling and Diagnosis of a Variable Speed PM Machines by Physics-based Modeling Approach," Presented at 14th International IGTE Symposium on numerical field calculation in electrical Eng., O4-3 pp. 84, IGTE2010 Graz, Austria, 2010. http://www.igte.tugraz.at/symp10/cms/images/stories/programme13092010.pdf.

- Ali Sarikhani and Osama A. Mohammed, "Non-Linear FE Physics-Based Modeling of Induction Machines For Real Time Integrated Drive Simulations," Presented at P2-03, Eight International Conf. on Computation in Electromagnetics, CEM2011, paper code 0069, April 2011.http://www.cem2011.com/pdfs/Abstracts\%20Session\%20P2.pdf.

- Ali Sarikhani, Wilder Saint-Hilaire, Osama A. Mohammed,“ A Study on the Effect of PM Machine Design Parameters Changes on Performance Measures," Presented at IEEE Power Engineering Society, PES2010, pp. 1-5, 2010, DOI:10.1109/PES.2010.5589743.

- Ali Sarikhani, M. Barzegaran, O. A. Mohammed, "Optimum Equivalent Models of Multi-Conductor Systems for the Study of Electromagnetic Signatures and radiated Emissions from Electric Drives," Presented at compumag 2011, paper number 503-1, 12-15 July 2011.

- Ali Sarikhani, O. A. Mohammed, "Hybrid GA-PSO Multi-Objective Design Optimization of Coupled PM Synchronous Motor-Drive Using Physics-Based Modeling Approach," Presented at IEEE Conf. on Electromagnetic field computations CEFC2010, PP. 1, DOI: 10.1109/CEFC.2010.5481775, 2010.

- A. Nejadpak, M. Barzegaran, A. Sarikhani, O. Mohammed“ Design of planar inductor based Z-source inverter for residential alternate energy sources," presented at Apec, pp. 1698 - 1703, 2011.

- M. R. Barzegaran, Ali Sarikhani, and Osama A. Mohammed, "Equivalent Current Loop Model for the Study of Radiated Electromagnetic Field Interference in Multi-Machine Electric Drives," proc. of 27th Annual Review of Progress in Applied Computational Electromagnetics, Williamsburg, Virginia , 2011 ACES, pp. 219-224, March 27-31, 2011.

- M. R. Barzegaran, Ali Sarikhani, and Osama A. Mohammed, “An Equivalent Source Model for the Study of Radiated Electromagnetic Fields in Multi-Machine Electric Drive Systems," proceeding of IEEE International Symposium on Electromagnetic Compatibility EMC, pp. 442, Aug. 2011. 
- Arash Nejadpak, Ali Sarikhani, Osama Mohammed, "Analysis of Radiated EMI and Noise Propagation in Three-Phase Inverter System Operating Under Different Switching Patterns, Accepted in CEFC2012. 


\section{APPENDICES}

\section{APPENDIX I \\ DESCRIPTION OF EXPERIMENTAL SETUPS}

The experimental set up of sensorless control of PM synchronous machine is depicted in figure I.1. The system includes a $0.25 \mathrm{~kW}, 44$ VDC surface mount permanent magnet synchronous machine. This machine is connected to a PM DC generator which acts as a load. The machine has incremental magnetic encoder. The measured rotor position was used as the reference for comparison. The three phase AC drive is supplied by a 44 DC power supply. The phase currents are measured by two current transducers. The employed switches in the drive are MOSFET-based which can carry up to 20 Amps. The nominal rating of the current was 10 Amps. The measured currents and rotor position are passed to the dSPACE1103 where the sensorless algorithm and speed control loops were applied. The software control-desk and Matlab/SIMULINK were used for sensorless and speed control loop implementations. The digital time step in sensorless calculations is $20(\mu s)$. The time step in speed control loops is $100(\mu s)$. The estimated rotor position is sampled down by the factor of ten before employing in the speed control loop.

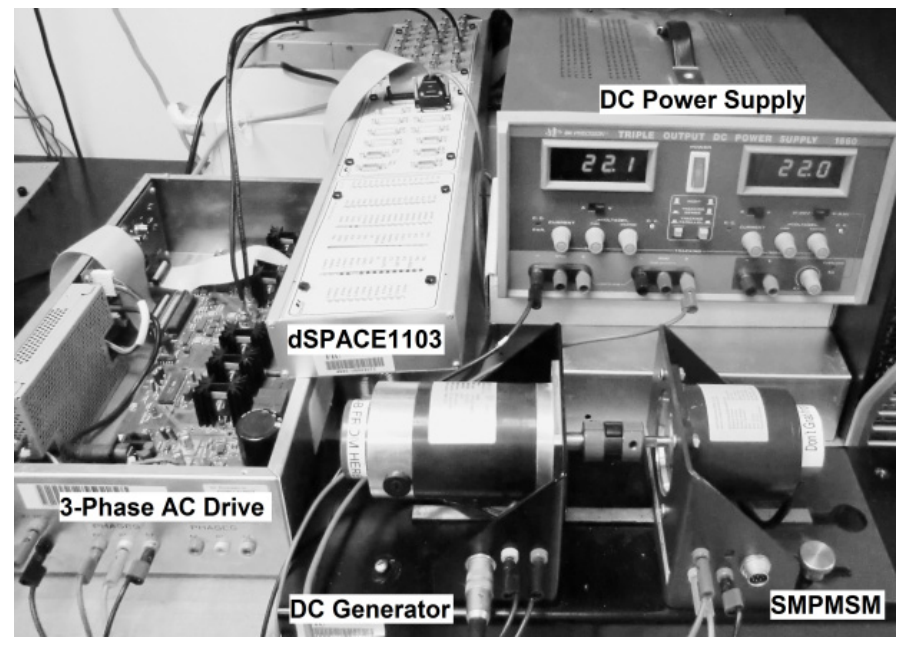

Figure I.1 Experimental set up for sensorless control of the machine 
The developed system for fault diagnosis system verifications is depicted in figure I.2. The system includes a $0.8 \mathrm{KW}, 48$ VDC surface mounted permanent magnet synchronous machine. The inter-turn short circuits were externally applied by connecting the turn's leads. The fault resistance is connected to the turn's lead. This machine is connected to a $0.25 \mathrm{KW}$ PM DC generator which acts as load. The machine has incremental magnetic encoder. The measured rotor position is used in variable speed drive controller. A DC power supply is used to supply the three phase AC drive. Two phase currents value are measured by two current transducers. The employed switches in the drive are MOSFET-based which can carry up to 20 Amps. The nominal rating of the current is 10 Amps. The measured currents and rotor position are passed to the SPACE1103 and to the dSPACE1104 for control purposes. The control circuit has an outer slow speed control loop and fast inner current control loop. The speed control loop provides the command torque and the current loops provide the reference direct and quadrature voltages. The DC bus voltage is typically measured by a voltage transducer from inside the drive DC bus. The software controldesk and Matlab/SIMULINK were used for diagnosis and speed control loop implementations. The digital time step in sensorless calculations is $20(\mu s)$. The time step in speed control loops is $100(\mu s)$.

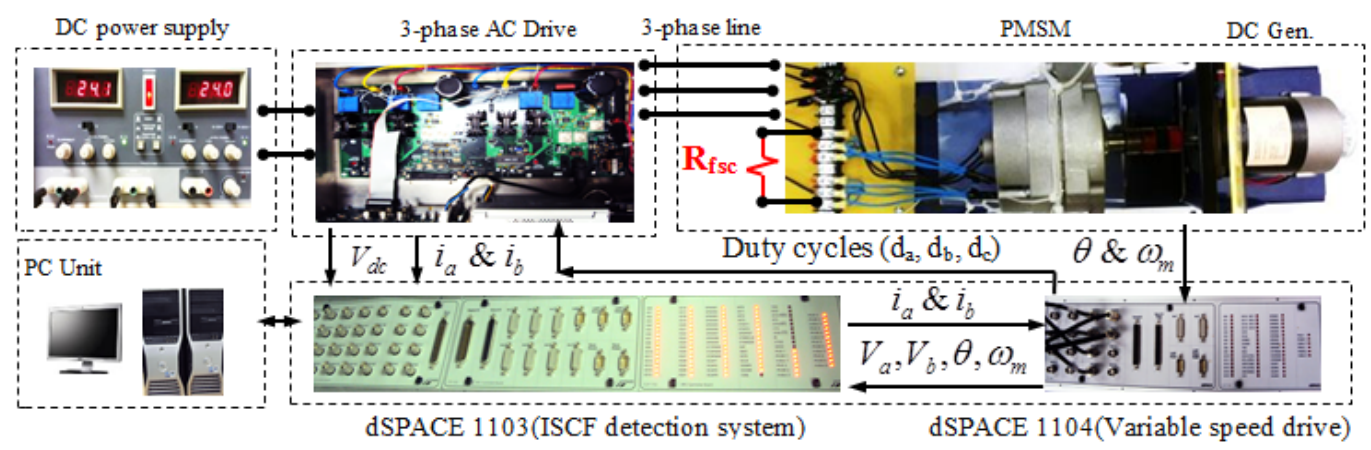

Figure I.2 Experimental set up for verification of inter-turn fault diagnosis system 
The developed system for hardware in the loop finite element based optimization process is depicted in figure I.3. The main body of software is implemented in MATLAB software in an M-file. The main optimization algorithm is implemented in the main body. The machine model is implemented is Simulink-MATLAB. The base software is enabled to invoke the Magnet-Infolytica finite element software. The Magnet-Infolytica finite element calculates the physical properties of the machine and it automatically sends it back to the main body. The main body software then renews the machine model inside the Simulink. After that the renewed model is built the on the dSPACE 1103. In order to increase the computational abilities, a dSPACE1104 is connected to dSPACE1103. The dSPACE1104 controls the AC/DC drive and the dSPACE 1103 handles the machine model. The digital time step in sensorless calculations is $20(\mu s)$. The time step in speed control loops is $100(\mu s)$.

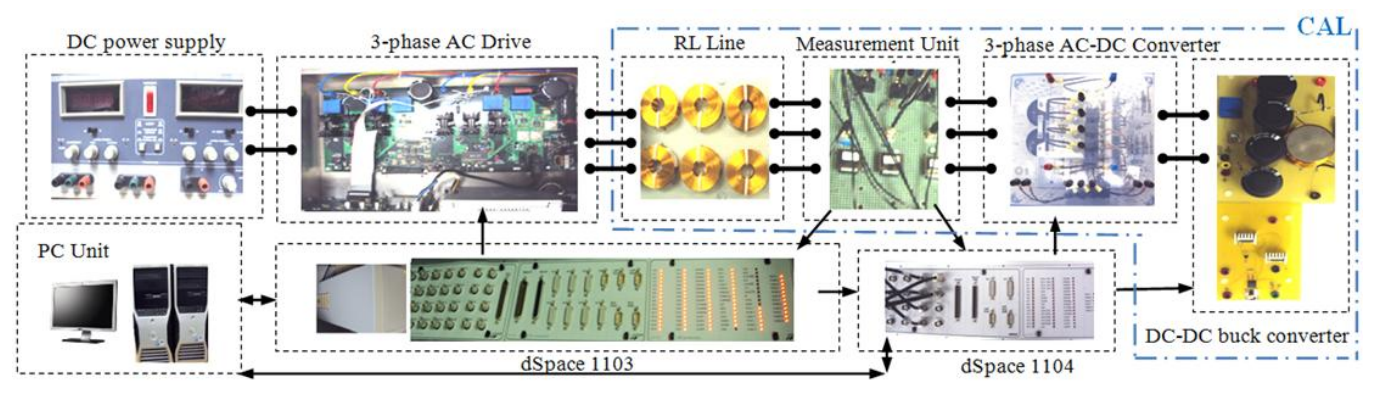

Figure I.3 Experimental set up for verification of HIL-finite element based design optimization 


\section{APPENDIX II}

Table II.1 Bbasic law of electromagnetism [202]

\begin{tabular}{l|l|l|}
\hline Ampere's law & $\nabla \times H=J$ & (II.1) \\
\hline Faraday's law & $\nabla \times E=-d B / d t$ \\
\hline Magnetic flux continuity & $\nabla \bullet B=0$ \\
\hline Permeability Law & $B=\mu . H$ \\
\hline Ohm's Law & $J=\sigma . E$ & (II.2) \\
\hline
\end{tabular}

